\title{
INTEGRAL MODELS OF SHIMURA VARIETIES WITH PARAHORIC LEVEL STRUCTURE
}

\author{
M. KISIN AND G. PAPPAS
}

\begin{abstract}
For a prime $p>2$, we construct integral models over $p$ for Shimura varieties with parahoric level structure, attached to Shimura data $(G, X)$ of abelian type, such that $G$ splits over a tamely ramified extension of $\mathbb{Q}_{p}$. The local structure of these integral models is related to certain "local models", which are defined group theoretically. Under some additional assumptions, we show that these integral models satisfy a conjecture of Kottwitz which gives an explicit description for the trace of Frobenius action on their sheaf of nearby cycles.
\end{abstract}

\section{Contents}

Introduction

1. Parahoric subgroups and minuscule representations

1.1. Bruhat-Tits and parahoric group schemes

1.2. Maps between Bruhat-Tits buildings

1.3. Minuscule representations and group schemes

1.4. Extending torsors

2. Local Models

2.1. The local models

2.2. Local models and central extensions.

2.3. Embedding local models in Grassmannians

3. Deformations of $p$-divisible groups

3.1. A construction for the universal deformation

3.2. Deformations with crystalline cycles

3.3. Deformations with étale cycles

4. Shimura varieties and local models

4.1. Shimura varieties of Hodge type

4.2. Integral models

4.3. Integral models for parahoric level

4.4. Twisting Abelian Varieties

4.5. The adjoint group action

4.6. Shimura varieties of abelian type

4.7. Nearby cycles

References

2000 Mathematics Subject Classification: Primary 11G18; Secondary 14G35

Date: April 16, 2018.

M. Kisin is partially supported by NSF grant DMS-1301921.

G. Pappas is partially supported by NSF grants DMS-1360733 and DMS-1701619. 


\section{INTRODUCTION}

The aim of this paper is to construct integral models for a large class of Shimura varieties with parahoric level structure, namely for those which are of abelian type, and such that the underlying group $G$ splits over a tamely ramified extension. Recall that $(G, X)$ is said to be of Hodge type if the corresponding Shimura variety can be described as a moduli space of abelian varieties equipped with a certain family of Hodge cycles. The Shimura data of abelian type is a larger class, which can be related to those of Hodge type. They include almost all cases where $G$ is a classical group. Our condition on the level structure allows many cases of Shimura varieties with non-smooth reduction at $p$.

One application of such models is to Langlands' program 49] to compute the Hasse-Weil zeta function of a Shimura variety in terms of automorphic $L$-functions. The zeta function has a local factor at $p$ which is determined by the $\bmod p$ points of the integral model, as well as its local structure - specifically the sheaf of nearby cycles. The integral models we construct are related to moduli spaces of abelian varieties (at least indirectly), which makes it feasible to count their mod $p$ points as in the work of Kottwitz [46] (cf. also 44]). On the other hand, their local structure is described in terms of "local models" which are simpler schemes given as orbit closures. In particular, we show that, when $G$ is unramified, the inertia acts unipotently on the sheaf of nearby cycles, and our models verify a conjecture of Kottwitz, which determines the (semi-simple) trace of Frobenius action on their nearby cycles rather explicitly. Such a local structure theory of integral models for Shimura varieties with parahoric level structure was first sought by Rapoport who took some of the first steps in extending the Langlands/Kottwitz method to the parahoric case 62, 63.

To state our results more precisely, let $p$ be a prime, and $(G, X)$ a Shimura datum. For $\mathrm{K}^{\circ} \subset G\left(\mathbb{A}_{f}\right)$ a neat, compact open subgroup, the corresponding Shimura variety

$$
\operatorname{Sh}_{K^{\circ}}(G, X)=G(\mathbb{Q}) \backslash X \times G\left(\mathbb{A}_{f}\right) / \mathrm{K}^{\circ}
$$

is naturally a scheme over the reflex field $\mathrm{E}=E(G, X)$, which does not depend on the choice of $\mathrm{K}^{\circ}$. Let $\mathrm{K}_{p}^{\circ} \subset G\left(\mathbb{Q}_{p}\right)$ be a parahoric subgroup, fix a compact open subgroup $\mathrm{K}^{p} \subset G\left(\mathbb{A}_{f}^{p}\right)$, and let $\mathrm{K}^{\circ}=\mathrm{K}_{p}^{\circ} \mathrm{K}^{p}$. We set

$$
\operatorname{Sh}_{\mathrm{K}_{p}^{\circ}}(G, X)=\lim _{\leftarrow \mathrm{K}^{p}} \operatorname{Sh}_{\mathrm{K}^{\circ}}(G, X) \text {. }
$$

Fix $v \mid p$ a prime of $\mathrm{E}$, let $E=\mathrm{E}_{v}$ and denote by $\kappa(v)$ the residue field of $E$. We say that a flat $\mathcal{O}_{E}$-scheme $S$ satisfies the extension property, if for any discrete valuation ring $R \supset \mathcal{O}_{E}$ of mixed characteristic $0, p$, the map $S(R) \rightarrow S(R[1 / p])$ is a bijection.

For the rest of the introduction, we assume that $p>2$, that $(G, X)$ is of abelian type, and that $G$ splits over a tamely ramified extension of $\mathbb{Q}_{p}$. We also assume that $p$ does not divide the order $\left|\pi_{1}\left(G^{\text {der }}\right)\right|$ of the (algebraic) fundamental group of the derived group $G^{\text {der }}$ over $\overline{\mathbb{Q}}_{p}$.

Proposition 0.1. The E-scheme $\operatorname{Sh}_{\mathrm{K}_{p}^{\circ}}(G, X)$ admits a $G\left(\mathbb{A}_{f}^{p}\right)$-equivariant extension to a flat $\mathcal{O}_{E}$-scheme $\mathscr{S}_{\mathrm{K}_{p}}(G, X)$, satisfying the extension property. Any sufficiently small compact open $\mathrm{K}^{p} \subset G\left(\mathbb{A}_{f}^{p}\right)$ acts freely on $\mathscr{S}_{\mathrm{K}_{p}^{\circ}}(G, X)$, and the quotient

$$
\mathscr{S}_{\mathrm{K}}(G, X):=\mathscr{S}_{\mathrm{K}_{p}^{\circ}}(G, X) / \mathrm{K}^{p}
$$

is a finite $\mathcal{O}_{E}$-scheme extending $\operatorname{Sh}_{\mathrm{K}_{p}^{\circ}}(G, X)_{E}$. 
In fact one can probably prove a result such as the proposition under weaker assumptions on $G$. The main point of our results is to describe the local structure of these models in terms of orbit closures when $G$ splits over a tamely ramified extension of $\mathbb{Q}_{p}$. To explain this, recall that the parahoric subgroup $\mathrm{K}_{p}^{\circ}$ is associated to a point of the building $x \in \mathcal{B}\left(G, \mathbb{Q}_{p}\right)$, which in turn defines, via the theory of Bruhat-Tits, a connected smooth group scheme $\mathcal{G}^{\circ}$ over $\mathbb{Z}_{p}$, whose generic fibre is $G$, and such that $\mathcal{G}^{\circ}\left(\mathbb{Z}_{p}\right) \subset G\left(\mathbb{Q}_{p}\right)$ is identified with $\mathrm{K}_{p}^{\circ}$. It had been conjectured by Rapoport [63, $\S 6,7]$ (see also [56]), that $\operatorname{Sh}_{K^{\circ}}(G, X)$ admits an integral model $\mathscr{S}_{\mathrm{K}} \circ(G, X)$, whose singularities are controlled by a "local model", $\mathrm{M}_{G, X}^{\text {loc }}$, with an explicit group theoretic description. Although a general definition of $\mathrm{M}_{G, X}^{\text {loc }}$ was not given in [63], it was conjectured that $\mathrm{M}_{G, X}^{\mathrm{loc}}$, should be equipped with an action of $\mathcal{G}^{\circ}$, and that there should be a smooth morphism of stacks

$$
\lambda: \mathscr{S}_{\mathrm{K} \circ}(G, X) \rightarrow\left[\mathcal{G}^{c, \circ} \backslash \mathrm{M}_{G, X}^{\mathrm{loc}}\right],
$$

which is to say a "local model diagram" consisting of maps of $\mathcal{O}_{E}$-schemes

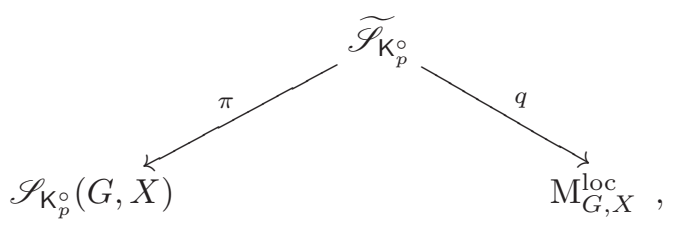

where $\pi$ is a $\mathcal{G}^{c, \circ}$-torsor, and $q$ is smooth and $\mathcal{G}^{c, \circ}$-equivariant. Here, $\mathcal{G}^{c, \circ}$ is the (smooth) quotient $1 \mathcal{G}^{\circ} / \mathcal{Z}_{s}$, where $\mathcal{Z}_{s}$ is the Zariski closure in $\mathcal{G}^{\circ}$ of the maximal $\mathbb{R}$-split but $\mathbb{Q}$-anisotropic subtorus of the center of $G$. This conjecture was inspired by a similar result for Shimura varieties of PEL type that first appeared in Deligne and one of us (G.P) [21], and de Jong [18] for special cases, and in the book of Rapoport-Zink 64 more generally. In particular, the work of Rapoport and Zink implies such a result for many Shimura varieties of PEL type with parahoric level structure but with an ad-hoc definition of $\mathrm{M}_{G, X}^{\text {loc }}$ given case-by-case. See the survey article [58] for more information and for additional references.

When $G$ splits over a tamely ramified extension a candidate for $\mathrm{M}_{G, X}^{\mathrm{loc}}$ was constructed in [59] by one of us (G.P) and Zhu. The construction of loc. cit. is reviewed in $\S 2$, and uses the affine Grassmannian for $G$. In $\S 2.3$ we show that it also has a more direct description as an orbit closure in a standard (i.e. not affine) Grassmannian. We show that these local models $\mathrm{M}_{G, X}^{\text {loc }}$ can be used to control the integral models $\mathscr{S}_{\mathrm{K}}(G, X)$ in Proposition 0.1 étale locally:

Theorem 0.2. If $\kappa / \kappa(v)$ is a finite extension, and $z \in \mathscr{S}_{\mathrm{K}^{\circ}}(G, X)(\kappa)$, then there exists $w \in \mathrm{M}_{G, X}^{\mathrm{loc}}\left(\kappa^{\prime}\right)$, with $\kappa^{\prime} / \kappa$ a finite extension, such that there is an isomorphism of strict henselizations

$$
\mathcal{O}_{\mathscr{S}_{\mathrm{K}}(G, X), z}^{\mathrm{sh}} \cong \mathcal{O}_{\mathrm{M}_{G, X}^{\mathrm{loc}}, w}^{\mathrm{sh}}
$$

The theorem, combined with results in [59], implies the following result about the local structure of $\mathscr{S}_{\mathrm{K}^{\circ}}(G, X)$.

Corollary 0.3. The special fibre $\mathscr{S}_{\mathrm{K}}(G, X) \otimes \kappa(v)$ is reduced, and the strict henselizations of the local rings on $\mathscr{S}_{\mathrm{K}}(G, X) \otimes \kappa(v)$ have irreducible components which are normal and Cohen-Macaulay.

\footnotetext{
${ }^{1}$ This quotient by $\mathcal{Z}_{s}$ is omitted in 63 and other references. If $(G, X)$ is of Hodge type, then $\mathcal{Z}_{s}=\{1\}$ and so $\mathcal{G}^{c, \circ}=\mathcal{G}^{\circ}$.
} 
If $\mathrm{K}_{p}^{\circ}$ is associated to a point $x$ which is a special vertex in $\mathcal{B}\left(G, \mathbb{Q}_{p}^{\text {ur }}\right)$, then $\mathscr{S}_{\mathrm{K}^{\circ}}(G, X) \otimes \kappa(v)$ is normal and Cohen-Macaulay.

We often obtain a more precise result, involving a slightly weaker form of the local model diagram:

Theorem 0.4. Suppose that either $\left(G^{\mathrm{ad}}, X^{\mathrm{ad}}\right)$ has no factor of type $D^{\mathbb{H}}$, or that $G$ is unramified over $\mathbb{Q}_{p}$ and $\mathrm{K}_{p}^{\circ}$ is contained in a hyperspecial subgroup. Then there exists a local model diagram

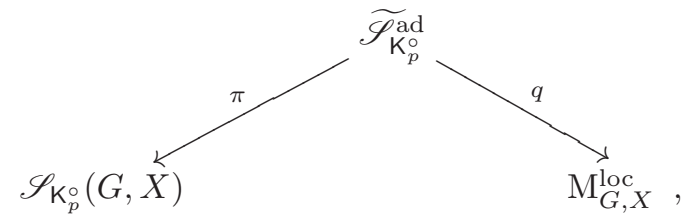

where $\pi$ is a $\mathcal{G}^{\text {ado }}$-torsor and $q$ is smooth and $\mathcal{G}^{\text {ado }}$-equivariant. In particular, for any $z \in \mathscr{S}_{\mathrm{K}^{\circ}}(G, X)(\kappa)$, there exists $w \in \mathrm{M}_{G, X}^{\mathrm{loc}}(\kappa)$ such that there is an isomorphism of henselizations $\mathcal{O}_{\mathscr{S}_{\mathrm{K}^{\circ}}(G, X), z}^{\mathrm{h}} \cong \mathcal{O}_{\mathrm{M}_{G, X}^{\mathrm{loc}}, w}^{\mathrm{h}}$.

Here, $\mathcal{G}^{\text {ado }}$ denotes the connected smooth group scheme with generic fibre $G^{\text {ad }}$ associated by Bruhat-Tits theory to the image $x^{\text {ad }}$ of the point $x$ under the canonical map $\mathcal{B}\left(G, \mathbb{Q}_{p}\right) \rightarrow \mathcal{B}\left(G^{\text {ad }}, \mathbb{Q}_{p}\right)$. Under our assumptions, $\mathcal{G}^{\text {ado }}$ also acts on $\mathrm{M}_{G, X}^{\text {loc }}$. In fact, the condition $p \nmid\left|\pi_{1}\left(G^{\text {der }}\right)\right|$ in the above theorem can be removed, although $\mathrm{M}_{G, X}^{\mathrm{loc}}$ then has to be replaced with a slightly different local model, attached to an auxiliary Shimura datum of Hodge type.

Below, we write $\mathscr{S}=\mathscr{S}_{\mathrm{K}^{\circ}}(G, X)$. Let $\bar{E}$ be an algebraic closure of $E$, with residue field $k_{\bar{E}}$, and $F \subset \bar{E}$ a subfield with $F / E$ finite and such that $G_{F}$ is split. The relationship with $\mathrm{M}_{G, X}^{\mathrm{loc}}$ and one of the results of [59], allows us to show the following result on the action of inertia on the sheaf of nearby cycles $R \Psi^{\mathscr{S}}$.

Corollary 0.5. For $\bar{z} \in \mathscr{S}\left(k_{\bar{E}}\right)$, the inertia subgroup $I_{F}$ of $\operatorname{Gal}(\bar{E} / F)$ acts unipotently on all the stalks $R \Psi_{\bar{z}}^{\mathscr{S}}$. If $\mathrm{K}_{p}^{\circ}$ is associated to a very special verte $x \in$ $\mathcal{B}\left(G, \mathbb{Q}_{p}\right)$, then $I_{F}$ acts trivially on all the stalks $R \Psi_{\bar{z}}^{\mathscr{S}}, \bar{z}$ as above.

In fact, we also give results about the semi-simple trace of Frobenius on the sheaf of nearby cycles of $\mathscr{S}_{\mathrm{K}^{\circ}}(G, X)$. Under the assumptions of Theorem 0.4 we show, again using results of [59], that this trace is given by a function which lies in the center of the parahoric Hecke algebra. When $G$ is unramified, we can deduce that $\mathscr{S}_{\mathrm{K}^{\circ}}(G, X)$ verifies a more precise conjecture of Kottwitz (see [36, §7]). This was first shown by Haines-Ngô for unramified unitary groups and for symplectic groups [37, and by Gaitsgory in the function field case [25]. Let us give some details. Since $G$ is unramified, $E$ is an unramified extension of $\mathbb{Q}_{p}$. We denote by $E_{r} / E$ the unramified extension of degree $r$, and by $\kappa_{r}$ its residue field. Suppose that $\mathrm{K}_{p}^{\circ} \subset G\left(\mathbb{Q}_{p}\right)$ is a parahoric subgroup, and set $P_{r}=\mathcal{G}^{\circ}\left(\mathcal{O}_{E_{r}}\right)$. Let $\mu$ be a cocharacter of $G$, in the conjugacy class of $\mu_{h}$, where $h \in X$. One has the associated Bernstein function $z_{\mu, r}$ in the center of the parahoric Hecke algebra $C_{c}\left(P_{r} \backslash G\left(E_{r}\right) / P_{r}\right)$.

\footnotetext{
${ }^{2}$ By definition [59], this means that $x$ is a special vertex in $\mathcal{B}\left(G, \mathbb{Q}_{p}\right)$ and is also special in $\mathcal{B}\left(G, \mathbb{Q}_{p}^{\text {ur }}\right)$. Such $x$ exist only when $G$ is quasi-split over $\mathbb{Q}_{p}$.
} 
Theorem 0.6. (Kottwitz's conjecture) Suppose that $G$ is unramified over $\mathbb{Q}_{p}$, and that either $\left(G^{\mathrm{ad}}, X^{\mathrm{ad}}\right)$ has no factor of type $D^{\mathbb{H}}$, or $\mathrm{K}_{p}^{\circ}$ is contained in a hyperspecial

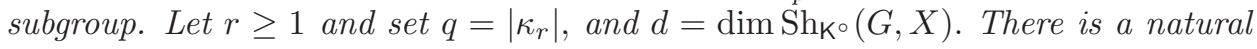
embedding

For $y \in \mathscr{S}\left(\kappa_{r}\right)$

$$
\mathcal{G}^{\circ}\left(\mathbb{F}_{q}\right) \backslash \mathrm{M}_{G, X}^{\mathrm{loc}}\left(\mathbb{F}_{q}\right) \hookrightarrow P_{r} \backslash G\left(E_{r}\right) / P_{r}
$$

where $w \in \mathrm{M}_{G, X}^{\text {loc }}\left(\kappa_{r}\right)$ corresponds to $y$ via the local model diagram.

We now explain the methods and organization of the paper in more detail. When $\mathrm{K}_{p}^{\circ}$ is hyperspecial the integral models $\mathscr{S}_{\mathrm{K}^{\circ}}(G, X)$ were constructed in [43] and, as expected, turn out to be smooth. However, for more general parahoric level structures $\mathrm{K}_{p}^{\circ}$, many of the key arguments of 43 ] break down or become much more complicated.

In the first section, we prove various results about the parahoric group schemes $\mathcal{G}^{\circ}$, and torsors over them. To explain these, consider a faithful minuscule representation $\rho: G \rightarrow \mathrm{GL}(V)$. In $\S 1.2$, we explicate a result of Landvogt [4], and show that $\rho$ induces a certain kind of embedding of buildings $\iota: \mathcal{B}\left(G, \mathbb{Q}_{p}\right) \hookrightarrow \mathcal{B}\left(\mathrm{GL}(V), \mathbb{Q}_{p}\right)$. This is then used to show in $\S 1.3$, that for $x \in \mathcal{B}\left(G, \mathbb{Q}_{p}\right)$, there is an closed embedding of group schemes $\mathcal{G}_{x} \rightarrow \mathcal{G} \mathcal{L}(V)_{\iota(x)}$. The existence of such an embedding is needed in exploiting Hodge cycles, to study integral models later in the paper. It replaces a general result for maps of reductive groups due to Prasad-Yu [61, which was used in 43 .

In $\S 1.4$, we show that a $\mathcal{G}^{\circ}$-torsor over the complement of the closed point in $\operatorname{Spec}\left(W\left(\mathbb{F}_{q}\right) \llbracket u \rrbracket\right)$ extends to $\operatorname{Spec}\left(W\left(\mathbb{F}_{q}\right) \llbracket u \rrbracket\right)$, and hence is trivial. As in $\llbracket 43$, this result is used in an essential way in showing that the crystalline realizations of certain Hodge cycles have good $p$-adic integrality properties, and eventually in relating the local models $\mathrm{M}_{G, X}^{\text {loc }}$ to the integral models $\mathscr{S}_{\mathrm{K}_{p}^{\circ}}(G, X)$. When $\mathcal{G}^{\circ}$ is reductive, this extension result was proved in [16], and is a simple consequence of the analogous extension result for vector bundles. For general parahorics $\mathcal{G}^{\circ}$, the proof becomes much more involved, and uses in particular results of Gille [28] and Bayer-Fluckiger - Parimala [2, 3] on Serre's conjecture II. In fact, for this reason we prove the result only when $G$ has no factors of type $E_{8}$.

In $\S 2$, we recall the construction of the local models $\mathrm{M}_{G, X}^{\text {loc }}$ introduced in [59]. Their definition involves the affine Grassmannian, however using the embedding $\iota$ mentioned above, we show that these local models can also be described as an orbit closure in a Grassmannian. This description is used in $\S 3$, to show that any formal neighborhood of a closed point of $\mathrm{M}_{G, X}^{\mathrm{loc}}$ supports a family of $p$-divisible groups, equipped with a family of crystalline cycles. More precisely, let $K / \mathbb{Q}_{p}$ be a finite extension, $\mathscr{G}$ a $p$-divisible group over $\mathcal{O}_{K}$, and $\left(s_{\alpha \text {,ét }) \subset T_{p} \mathscr{G} \otimes}\right.$ a family of Galois invariant tensors in the Tate module $T_{p} \mathscr{G}$, whose pointwise stabilizer can be identified with the parahoric group scheme $\mathcal{G}^{\circ} \subset \mathrm{GL}\left(T_{p} \mathscr{G}\right)$ (in fact we deal also with non-connected stabilizers). If $\mathbb{D}$ denotes the Dieudonné module of $\mathscr{G}$, then the crystalline counterparts of the $\left(s_{\alpha \text {,ét }}\right)$ are tensors $\left(s_{\alpha, 0}\right) \subset \mathbb{D}[1 / p]^{\otimes}$. Using the extension result of $\S 1.4$, mentioned above, we show that $\left(s_{\alpha, 0}\right) \subset \mathbb{D}^{\otimes}$ and that these tensors define a parahoric subgroup of $G L(\mathbb{D})$ which is isomorphic to $\mathcal{G}^{\circ}$. This allows us to construct the required family of $p$-divisible groups over a formal neighborhood of $\mathrm{M}_{G, X}^{\mathrm{loc}}$. In [43] this was done using an explicit construction of the 
universal deformation, due to Faltings. However this construction does not seem to generalize to the parahoric case, and we use instead a construction involving Zink's theory of displays [75] $(\S 3.1,3.2)$.

Finally in $\S 4$, we apply all this to integral models of Shimura varieties. We use the families of $p$-divisible groups over formal neighborhoods of $\mathrm{M}_{G, X}^{\mathrm{loc}}$, to relate $\mathrm{M}_{G, X}^{\text {loc }}$ and $\mathscr{S}_{\mathrm{K}_{p}^{\circ}}(G, X)$, when $(G, X)$ is of Hodge type. In particular, these results also cover the PEL cases of 64 and our proof then circumvents the complicated case-by-case linear algebra arguments with lattice chains in loc. cit., Appendix. (In some sense, the role of these linear algebra arguments is now played by the extension result of §1.4.) To extend these results to the case of abelian type Shimura data, we follow Deligne's strategy [20, using connected Shimura varieties and the action of $G^{\text {ad }}(\mathbb{Q})^{+}$. As in 43 we use a moduli theoretic description of this action, in terms of a kind of twisting of abelian schemes. In the final subsection, we give the application to nearby cycles and Kottwitz's conjecture.

The application to integral models is somewhat complicated by the phenomenon that for $x \in \mathcal{B}\left(G, \mathbb{Q}_{p}\right)$, the stabilizer group scheme $\mathcal{G}_{x}$, attached to $x$ by BruhatTits, may not have connected special fibre. On the one hand, it is more convenient to work with the connected component of the identity $\mathcal{G}_{x}^{\circ}$; for example, the local model diagram in Theorem 0.4 yields an isomorphism of henselizations only when $\pi$ is a torsor under a smooth connected group (using Lang's lemma). On the other hand, our arguments with Hodge cycles yield direct results only for integral model with level $\mathrm{K}_{p}=\mathcal{G}_{x}\left(\mathbb{Z}_{p}\right)$. We are able to overcome these difficulties in most, but not quite all cases, and this is the reason for the restriction on $G$ in Theorem 0.4

Acknowledgment. We would like to thank T. Haines, K.-W. Lan, G. Prasad, M. Rapoport and R. Zhou for a number of useful suggestions.

\section{Parahoric SubGroups and minuscule Representations}

\subsection{Bruhat-Tits and parahoric group schemes.}

1.1.1. Let $p$ be a prime number. If $R$ is an algebra over the $p$-adic integers $\mathbb{Z}_{p}$, we will denote by $W(R)$ the ring of Witt vectors with entries in $R$. Let $k$ be either a finite extension of $\mathbb{F}_{p}$ or an algebraic closure of $\mathbb{F}_{p}$. Let $\bar{k}$ be an algebraic closure of $k$. We set $W=W(k), K_{0}=\operatorname{Frac}(W)$, and $L=\operatorname{Frac} W(\bar{k})$.

In what follows, we let $K$ be either a finite totally ramified field extension of $K_{0}$, or the equicharacteristic local field $k((\pi))$ of Laurent power series with coefficients in $k$. We let $\bar{K}$ be an algebraic closure of $K$ with residue field $\bar{k}$. We denote by $K^{\text {ur }} \subset \bar{K}$ the maximal unramified extension of $K$ in $\bar{K}$, and we write $\mathcal{O}=\mathcal{O}_{K}$ and $\mathcal{O}^{\text {ur }}=\mathcal{O}_{K^{\text {ur }}}$ for the valuation rings of $K$ and $K^{\text {ur }}$.

1.1.2. Let $G$ be a connected reductive group over $K$. We will denote by $\mathcal{B}(G, K)$ the (extended) Bruhat-Tits building of $G(K)$ 10, 11, 70. We will also consider the building $\mathcal{B}\left(G^{\text {ad }}, K\right)$ of the adjoint group; the central extension $G \rightarrow G^{\text {ad }}$ induces a natural $G(K)$-equivariant map $\mathcal{B}(G, K) \rightarrow \mathcal{B}\left(G^{\text {ad }}, K\right)$ which is a bijection when $G$ is semi-simple. In particular, we can identify $\mathcal{B}\left(G^{\text {der }}, K\right)$ with $\mathcal{B}\left(G^{\text {ad }}, K\right)$.

If $\Omega$ is a non-empty bounded subset of $\mathcal{B}(G, K)$ which is contained in an apartment, we will write $G(K)_{\Omega}=\{g \in G(K) \mid g \cdot x=x, \forall x \in \Omega\}$ for the pointwise stabilizer ("fixer") of $\Omega$ in $G(K)$ and denote by $G(K)_{\Omega}^{\circ}$ the "connected stabilizer" ([11, $\S 4]$ ). When $\Omega=\{x\}$ is a point, $G(K)_{x}^{\circ}$ is, by definition, the parahoric subgroup of $G(K)$ that corresponds to $x$. Similarly, if $\Omega$ is an open facet, $G(K)_{\Omega}^{\circ}$ is 
the parahoric subgroup that corresponds to the facet $\Omega$. If $\Omega$ is an open facet and $x \in \Omega$, then $G(K)_{\Omega}^{\circ}=G(K)_{x}^{\circ}$.

Similarly, we can consider $G\left(K^{\mathrm{ur}}\right), G\left(K^{\mathrm{ur}}\right)_{\Omega}$ and $G\left(K^{\mathrm{ur}}\right)_{\Omega}^{\circ}$. By the main result of 11, there is a smooth affine group scheme $\mathcal{G}_{\Omega}$ over $\operatorname{Spec}(\mathcal{O})$ with generic fibre $G$ which is uniquely characterized by the property that $\mathcal{G}_{\Omega}\left(\mathcal{O}^{\mathrm{ur}}\right)=G\left(K^{\mathrm{ur}}\right)_{\Omega}$. By definition, we have $G\left(K^{\text {ur }}\right)_{\Omega}^{\circ}=\mathcal{G}_{\Omega}^{\circ}\left(\mathcal{O}^{\text {ur }}\right)$, where $\mathcal{G}_{\Omega}^{\circ}$ is the connected component of $\mathcal{G}_{\Omega}$. We will call $\mathcal{G}_{x}^{\circ}$ a "parahoric group scheme" (so these are, by definition, connected). More generally, we will call $\mathcal{G}_{\Omega}$ a "Bruhat-Tits group scheme" (even if $\Omega$ is not a facet).

Denote by $\bar{\Omega} \subset \mathcal{B}\left(G^{\text {ad }}, K\right)$ the image of $\Omega$ under $\mathcal{B}(G, K) \rightarrow \mathcal{B}\left(G^{\text {ad }}, K\right)$. We can then also consider the subgroup $G(K)_{\bar{\Omega}} \subset G(K)$ fixing $\bar{\Omega}$. We have $G(K)_{\Omega} \subset$ $G(K)_{\bar{\Omega}}$. By [38, Prop. 3 and Remarks 4 and 11], $G\left(K^{\mathrm{ur}}\right)_{\Omega}^{\circ}$ is the intersection of $G\left(K^{\mathrm{ur}}\right)_{\bar{\Omega}}$ (and hence, also of $G\left(K^{\mathrm{ur}}\right)_{\Omega}$ ) with the kernel $G\left(K^{\mathrm{ur}}\right)_{1}$ of the Kottwitz homomorphism $\kappa_{G}: G\left(K^{\mathrm{ur}}\right) \rightarrow \pi_{1}(G)_{I}$. It then follows that $G(K)_{\Omega}^{\circ}$ is also the intersection of $G(K)_{\Omega}$ with the kernel of the Kottwitz homomorphism. As a result, using [11, (1.7.6)], we see that $\mathcal{G}_{x}^{\circ}$ only depends on $G$ and the image $\bar{x}$ of $x$ in $\mathcal{B}\left(G^{\text {ad }}, K\right)$.

If $G$ is semi-simple, simply connected, then $\kappa_{G}$ is trivial and we have $G(K)_{\Omega}^{\circ}=$ $G(K)_{\Omega}$.

1.1.3. We continue with the notations of the previous paragraph. Let $\alpha: G \rightarrow \tilde{G}$ be a central extension between connected reductive groups over $K$ with kernel $Z$. By [11, (4.2.15)], or [48, Theorem 2.1.8], $\alpha$ induces a canonical $G(K)$-equivariant $\operatorname{map} \alpha_{*}: \mathcal{B}(G, K) \rightarrow \mathcal{B}(\tilde{G}, K)$. Set $\tilde{x}=\alpha_{*}(x)$. Then $\alpha\left(G\left(K^{\mathrm{ur}}\right)_{x}\right) \subset \tilde{G}\left(K^{\mathrm{ur}}\right)_{\tilde{x}}$ and, by [11, (1.7.6)], $\alpha$ extends to group scheme homomorphisms

$$
\alpha: \mathcal{G}_{x} \rightarrow \tilde{\mathcal{G}}_{\tilde{x}}, \quad \alpha: \mathcal{G}_{x}^{\circ} \rightarrow \tilde{\mathcal{G}}_{\tilde{x}}^{\circ} .
$$

We record the following for future use:

Proposition 1.1.4. Suppose that $G$ splits over a tamely ramified extension of $K$ and that $Z$ is either a torus or is finite of rank prime to $p$. Then the schematic closure $\mathcal{Z}$ of $Z$ in $\mathcal{G}_{x}^{\circ}$ is smooth over $\operatorname{Spec}(\mathcal{O})$ and it fits in an (fppf) exact sequence

$$
1 \rightarrow \mathcal{Z} \rightarrow \mathcal{G}_{x}^{\circ} \stackrel{\alpha}{\rightarrow} \tilde{\mathcal{G}}_{\tilde{x}}^{\circ} \rightarrow 1
$$

of group schemes over $\operatorname{Spec}(\mathcal{O})$. If $Z$ is a torus which is a direct summand of an induced torus, then $\mathcal{Z}=\mathcal{Z}^{\circ}$ is the connected Neron model of $Z$.

Proof. By base change, it is enough to show the Proposition when $k$ is algebraically closed. Then both $G$ and $\tilde{G}$ are quasi-split by Steinberg's theorem, and by our assumption, they split after a tame finite Galois extension $K^{\prime} / K$. Set $\Gamma=\operatorname{Gal}\left(K^{\prime} / K\right)$ which is a cyclic group.

Choose a maximal split torus in $G$ whose apartment contains $x$, and let $T$ be its centralizer. Since $G$ is quasi-split, $T$ is a maximal torus and we have an exact sequence

$$
1 \rightarrow Z \rightarrow T \stackrel{\alpha}{\rightarrow} \tilde{T} \rightarrow 1
$$

with $\tilde{T}$ a maximal torus in $\tilde{G}$. The central morphism $\alpha: G \rightarrow \tilde{G}$ induces an isomorphism between corresponding root subgroups $U_{a}$ and $\tilde{U}_{a}$. Denote by $\mathcal{U}_{a}$ and $\tilde{\mathcal{U}}_{a}$ their corresponding schematic closures in $\mathcal{G}_{x}^{\circ}$ and $\tilde{\mathcal{G}}_{\tilde{x}}^{\circ}$ respectively. By [11, (4.2.15), (4.3.2)], and the construction of $\mathcal{G}_{x}^{\circ}$ and $\tilde{\mathcal{G}}_{\tilde{x}}^{\circ}$ in [11, §4.6], the morphism $\alpha$ induces an isomorphism between $\mathcal{U}_{a}(\mathcal{O})$ and $\tilde{\mathcal{U}}_{a}(\mathcal{O})$, and therefore, by [11, (1.7.6)], 
between $\mathcal{U}_{a}$ and $\tilde{\mathcal{U}}_{a}$. Also, by [11, $\left.\S 4.4, \S 4.6\right]$, the schematic closure of $T$, resp. $\tilde{T}$, in $\mathcal{G}_{x}^{\circ}$, resp. $\tilde{\mathcal{G}}_{\tilde{x}}^{\circ}$, is the connected Neron model $\mathcal{T}^{\circ}$, resp. $\tilde{\mathcal{T}}^{\circ}$ of $T$, resp. $\tilde{T}$. Assume we have an fppf exact sequence

$$
1 \rightarrow \mathcal{Z} \rightarrow \mathcal{T}^{\circ} \stackrel{\alpha}{\rightarrow} \tilde{\mathcal{T}}^{\circ} \rightarrow 1
$$

where $\mathcal{Z}$ is smooth and is the schematic closure of $Z$ in $\mathcal{T}^{\circ}$. Then $\mathcal{Z}$ is also the schematic closure of $Z$ in $\mathcal{G}_{x}^{\circ}$ and the quotient $\mathcal{G}_{x}^{\circ} / \mathcal{Z}$, which is representable by [1, §4], is a connected smooth group scheme which admits a homomorphism $\gamma$ : $\mathcal{G}_{x}^{\circ} / \mathcal{Z} \rightarrow \tilde{\mathcal{G}}_{\tilde{x}}^{\circ}$. Using again the construction of the parahoric group schemes via schematic root data ([11, §3], [11, §4.6] for the quasi-split case), we see that $\gamma$ is an isomorphism on an open neighborhood of the identity given by the "open big cell". By [11, (1.2.13)], $\gamma$ is an isomorphism and this proves the Proposition.

It remains to exhibit the exact sequence (1.1.6).

If $Z$ is a torus the desired statement follows by the argument in the proof of [57, Lemma 6.7] which gives the analogous result in the equal characteristic case.

Suppose that $Z$ is finite of rank prime to $p$. By base changing to $K^{\prime}$ we obtain $1 \rightarrow Z^{\prime} \rightarrow T^{\prime} \rightarrow \tilde{T}^{\prime} \rightarrow 1$ with $T^{\prime}, \tilde{T}^{\prime}$ split over $K^{\prime}$; here the prime indicates base change extension to $K^{\prime}$. This extends to an exact sequence of group schemes

$$
1 \rightarrow \mathcal{Z}^{\prime} \rightarrow \mathcal{T}^{\prime} \rightarrow \tilde{\mathcal{T}}^{\prime} \rightarrow 1
$$

with $\mathcal{T}^{\prime}, \tilde{\mathcal{T}}^{\prime}$ split tori over $\mathcal{O}^{\prime}$. Then $\mathcal{Z}^{\prime}=A(1)$, a finite multiplicative group scheme with $\Gamma$-action which is the Zariski closure of $Z^{\prime}$ in $\mathcal{T}^{\prime}$. (Here $A$ is a finite abelian group with $\Gamma$-action and $A(1)=A \otimes_{\mathbb{Z}} \mu_{n}$, for $n=\exp (A), n$ prime to $p$.) As $p \nmid n$, we can see, using Hensel's lemma, that we have an exact sequence of smooth group schemes

$$
1 \rightarrow \operatorname{Res}_{\mathcal{O}^{\prime} / \mathcal{O}}(A(1)) \rightarrow \operatorname{Res}_{\mathcal{O}^{\prime} / \mathcal{O}}\left(\mathcal{T}^{\prime}\right) \rightarrow \operatorname{Res}_{\mathcal{O}^{\prime} / \mathcal{O}}\left(\tilde{\mathcal{T}}^{\prime}\right) \rightarrow 1 .
$$

By taking the $\Gamma$-fixed (closed) subschemes we obtain the exact sequence

$$
1 \rightarrow \operatorname{Res}_{\mathcal{O}^{\prime} / \mathcal{O}}(A(1))^{\Gamma} \rightarrow \operatorname{Res}_{\mathcal{O}^{\prime} / \mathcal{O}}\left(\mathcal{T}^{\prime}\right)^{\Gamma} \rightarrow \operatorname{Res}_{\mathcal{O}^{\prime} / \mathcal{O}}\left(\tilde{\mathcal{T}}^{\prime}\right)^{\Gamma}
$$

Since $\# \Gamma$ is prime to $p$, by [22, Prop. 3.1], these fixed point (closed) subgroup schemes are also smooth over $\mathcal{O}$. The neutral components $\mathcal{T}^{\circ}$ and $\tilde{\mathcal{T}}^{\circ}$ of $\mathcal{T}:=$ $\operatorname{Res}_{\mathcal{O}^{\prime} / \mathcal{O}}\left(\mathcal{T}^{\prime}\right)^{\Gamma}$ and $\tilde{\mathcal{T}}:=\operatorname{Res}_{\mathcal{O}^{\prime} / \mathcal{O}}\left(\tilde{\mathcal{T}}^{\prime}\right)^{\Gamma}$ are the connected Neron models of $T$ and $\tilde{T}$ respectively.

Since $\mathcal{O}$ is strictly henselian, taking $\mathcal{O}$-valued points on 1.1.7 is exact. Using this together with the long exact sequence of $\Gamma$-cohomology gives an exact sequence

$$
0 \rightarrow A^{\Gamma} \rightarrow \mathcal{T}(\mathcal{O}) \rightarrow \tilde{\mathcal{T}}(\mathcal{O}) \rightarrow \mathrm{H}^{1}(\Gamma, A) .
$$

Since $\mathrm{H}^{1}(\Gamma, A)$ is finite, $\mathcal{T} \rightarrow \tilde{\mathcal{T}}$ has open image, and induces a surjection $\mathcal{T}^{\circ} \rightarrow \tilde{\mathcal{T}}^{\circ}$ between neutral components. Finally $\mathcal{Z}=\operatorname{ker}\left(\mathcal{T}^{\circ} \rightarrow \tilde{\mathcal{T}}^{\circ}\right)$ is open in $\operatorname{ker}(\mathcal{T} \rightarrow \tilde{\mathcal{T}})$, and hence étale, which completes the construction of (1.1.6).

Remark 1.1.8. Using similar arguments as above, we can also see that, under the assumptions of Proposition 1.1.4 the schematic closure of $Z$ in $\mathcal{G}_{x}$ is smooth over $\operatorname{Spec}(\mathcal{O})$ and is equal to the kernel of $\alpha: \mathcal{G}_{x} \rightarrow \tilde{\mathcal{G}}_{\tilde{x}}$. In general, $\alpha: \mathcal{G}_{x} \rightarrow \tilde{\mathcal{G}}_{\tilde{x}}$ is not fppf surjective; this happens, for example, when $\mathcal{G}_{x}=\mathcal{G}_{x}^{\circ}$ but $\tilde{\mathcal{G}}_{\tilde{x}} \neq \tilde{\mathcal{G}}_{\tilde{x}}^{\circ}$.

1.1.9. The building $\mathcal{B}(\mathrm{GL}(V), K)$ : Suppose that $V$ is a finite dimensional $K$-vector space. By [12, Prop. 1.8, Th. 2.11], the points of the building $\mathcal{B}(\mathrm{GL}(V), K)$ are in 1-1 correspondence with graded periodic lattice chains $(\{\Lambda\}, c)$ : By definition, 
a periodic lattice chain is a non-empty set of $\mathcal{O}$-lattices $\{\Lambda\}$ in $V$ which is totally ordered by inclusion and stable under multiplication by scalars. A grading $c$ is a strictly decreasing function $c:\{\Lambda\} \rightarrow \mathbb{R}$ which satisfies

$$
c\left(\pi^{n} \Lambda\right)=c(\Lambda)+n
$$

where $\pi$ is a uniformizer of $\mathcal{O}$. One can check (loc. cit.) that there is an integer $r \geq 1$ (the period) and distinct lattices $\Lambda^{i}$, for $i=0, \ldots, r-1$, such that

$$
\pi \Lambda^{0} \subset \Lambda^{r-1} \subset \cdots \subset \Lambda^{1} \subset \Lambda^{0}
$$

and $\{\Lambda\}=\left\{\Lambda^{i}\right\}_{i \in \mathbb{Z}}$, with $\Lambda^{j}$ defined by $\Lambda^{m r+i}=\pi^{m} \Lambda^{i}$ for $m \in \mathbb{Z}, 0 \leq i<r$.

The stabilizer $\mathrm{GL}(V)_{x}$ of the point $x \in \mathcal{B}(\mathrm{GL}(V), K)$ that corresponds to $(\{\Lambda\}, c)$ is the intersection $\bigcap_{i=0}^{r-1} \operatorname{GL}\left(\Lambda^{i}\right)$ in $\operatorname{GL}(V)$. By loc. cit. 3.8, 3.9, the corresponding parahoric group scheme $\mathcal{G} \mathcal{L}_{x}$ is the Zariski closure of the diagonally embedded $\mathrm{GL}(V) \hookrightarrow \prod_{i=0}^{r-1} \mathrm{GL}(V)$ in the product $\prod_{i=0}^{r-1} \mathrm{GL}\left(\Lambda^{i}\right)$. The group scheme $\mathcal{G} \mathcal{L}_{x}$ can also be identified with the group scheme of automorphisms $\operatorname{Aut}\left(\Lambda^{\bullet}\right)$ of the (indexed) lattice chain $\Lambda^{\bullet}:=\left\{\Lambda^{i}\right\}_{i \in \mathbb{Z}}$. This is true since this group of automorphisms is smooth (by [64, Appendix to Ch. 3]) and has the same $\mathcal{O}^{\text {ur }}$-valued points as $\mathcal{G} \mathcal{L}_{x}$. In fact, in [12, one finds a similar description of the building $\mathcal{B}\left(\mathrm{GL}(V)_{D}, K\right)$ and the parahoric subgroups when $V$ is a finite dimensional (right) $D$-module, where $D$ is a finite dimensional $K$-central division algebra. For $x \in \mathcal{B}\left(\operatorname{GL}(V)_{D}, K\right)$, we will denote by $\left(\mathcal{G L}(V)_{D}\right)_{x}$ the corresponding parahoric group scheme.

Note here that to simplify notation we will use the symbol GL $(\Lambda)$ to denote both the abstract group and the corresponding group scheme over $\operatorname{Spec}(\mathcal{O})$; this should not lead to confusion.

1.1.11. The building $\mathcal{B}(\operatorname{GSp}(V), K)$ : Suppose that $V$ is a finite dimensional $K$ vector space with a perfect alternating bilinear form $\psi: V \times V \rightarrow K$. There is an involution on the set of $\mathcal{O}$-lattices in $V$ given by $\Lambda \mapsto \Lambda^{\vee}:=\{v \in V \mid \psi(v, x) \in$ $\mathcal{O}, \forall x \in \Lambda\}$. In this case, the points of the building $\mathcal{B}(\operatorname{GSp}(V), K)$ are in 1-1 correspondence with "almost self-dual" graded period lattice chains $(\{\Lambda\}, c)$ and so $\mathcal{B}(\mathrm{GSp}(V), K) \subset \mathcal{B}(\mathrm{GL}(V), K)$. (This is a variant of a special case of the results of 13 that describe $\mathcal{B}(\operatorname{Sp}(V), K)$.) Here, almost self-dual means that the set $\{\Lambda\}$ is stable under the involution and that $c\left(\Lambda^{\vee}\right)=-c(\Lambda)+m$ for some $m \in \mathbb{Z}$, independent of $\Lambda$. In this case, there is an integer $r \geq 1$ and distinct lattices $\Lambda^{i}$, for $i=0, \ldots, r-1$, such that

$$
\Lambda^{r-1} \subset \cdots \subset \Lambda^{0} \subset\left(\Lambda^{0}\right)^{\vee} \subset \cdots \subset\left(\Lambda^{r-1}\right)^{\vee} \subset \pi^{-1} \Lambda^{r-1},
$$

and for $a=0$, or 1 , we have $\left(\Lambda^{i}\right)^{\vee}=\Lambda^{-i-a}$ for each $i$. The complete chain $\{\Lambda\}$ consists of all scalar multiples of these lattices $\Lambda^{i}$ and $\left(\Lambda^{i}\right)^{\vee}$. The stabilizer $\operatorname{GSp}(V)_{x}$ of the point $x \in \mathcal{B}(\operatorname{GSp}(V), K)$ that corresponds to $(\{\Lambda\}, c)$ is $\operatorname{GSp}(V) \cap$ $\mathrm{GL}(V)_{x}$. The corresponding parahoric group scheme $\mathcal{G S P}_{x}$ is the schematic closure of the diagonally embedded $\operatorname{GSp}(V) \hookrightarrow \prod_{i=-(r-1)-a}^{r-1} \mathrm{GL}(V)$ in the product $\prod_{i=-(r-1)-a}^{r-1} \operatorname{GL}\left(\Lambda^{i}\right)$. As above, by [64, Appendix to Ch. 3], this identifies with the group scheme of similitude automorphisms $\operatorname{Aut}\left(\left\{\Lambda^{i}\right\}_{i \in \mathbb{Z}}, \psi_{i}\right)$ of the polarized lattice chain. Here $\psi_{i}: \Lambda^{i} \times \Lambda^{-i-a} \rightarrow \mathcal{O}$ are the perfect alternating forms given by $\psi$ and we consider automorphisms that respect the $\psi_{i}$ up to common similitude.

Consider $V^{\prime}=\oplus_{i=-(r-1)-a}^{r-1} V$ equipped with the perfect alternating $K$-bilinear form $\psi^{\prime}: V^{\prime} \times V^{\prime} \rightarrow K$ given as the orthogonal direct sum $\perp_{i=-(r-1)-a}^{r-1} \psi$. We have a natural "diagonal" embedding $\operatorname{GSp}(V, \psi) \hookrightarrow \operatorname{GSp}\left(V^{\prime}, \psi^{\prime}\right) \subset \operatorname{GL}\left(V^{\prime}\right)$. Consider 
the lattice $\Lambda^{\prime}=\oplus_{i=-(r-1)-a}^{r-1} \Lambda^{i} \subset V^{\prime}$. Then, by the above, the group scheme $\mathcal{G S P}_{x}$ is the schematic closure of $\operatorname{GSp}(V, \psi)$ in $\operatorname{GL}\left(\Lambda^{\prime}\right)$. By replacing $\Lambda^{\prime}$ by a scalar multiple, we can assume that $\psi^{\prime}$ takes integral values on $\Lambda^{\prime}$, i.e. that $\Lambda^{\prime} \subset \Lambda^{\prime \vee}$ where the dual is with respect to $\psi^{\prime}$.

\subsection{Maps between Bruhat-Tits buildings.}

1.2.1. In this section, we elaborate on Landvogt's results 48 on embeddings of Bruhat-Tits buildings induced by (faithful) representations $\rho: G \rightarrow \operatorname{GL}(V)$. Here faithful means that the kernel of $\rho$ is trivial. Then $\rho$ gives a closed immersion of group schemes over $K$ (see for example [17, Theorem 5.3.5]). Landvogt shows that such a $\rho$ induces a $G(K)$-equivariant "toral" isometric embedding $\mathcal{B}(G, K) \rightarrow$ $\mathcal{B}(\mathrm{GL}(V), K)$ (see loc. cit. for the definition of toral); such an embedding is not uniquely determined by $\rho$ but also depends on the choice of the image of a given special point in $\mathcal{B}(G, K)$. In this section we give a more specific construction of such an embedding when $\rho$ is minuscule, see below. This construction will be used in 2.3 for showing that local models embed in certain Grassmannians.

1.2.2. First suppose that $G$ is split over $K$; denote by $G_{\mathcal{O}}$ a reductive model over $\mathcal{O}$. Let $x_{0}$ be a hyperspecial vertex of the building $\mathcal{B}(G, K)$ with stabilizer the hyperspecial subgroup $G_{\mathcal{O}}(\mathcal{O})$. Recall that there is canonical embedding $\mathcal{B}(G, K) \hookrightarrow \mathcal{B}\left(G, K^{\mathrm{ur}}\right)$ and we can also think of $x_{o}$ as a hyperspecial vertex of $\mathcal{B}\left(G, K^{\text {ur }}\right)$.

Suppose $\rho: G \rightarrow \mathrm{GL}(V)$ is a representation defined over $K$ (not necessarily faithful). Suppose we can write $V=\oplus_{i} V_{i}$, where for each $i, \rho_{i}: G \rightarrow \operatorname{GL}\left(V_{i}\right)$ is a $K$-representation which is irreducible and hence, since $G$ is split, also geometrically irreducible. Notice here that $\rho_{i}$ factors

$$
G \stackrel{a_{i}}{\longrightarrow} G_{i} \hookrightarrow \mathrm{GL}\left(V_{i}\right)
$$

where $a_{i}$ is an epimorphism. If $K=k((\pi))$, we assume that $a_{i}$, for each $i$, induces a separable morphism between each root subgroup of $G$ and its image in $G_{i}$. Here $G_{i}$ is also a split reductive group. Suppose that, for each $i, \Lambda_{i} \subset V_{i}$ is an $\mathcal{O}$-lattice such that

$$
\rho_{i}\left(G_{\mathcal{O}}\left(\mathcal{O}^{\text {ur }}\right)\right) \subset \mathrm{GL}\left(\Lambda_{i} \otimes_{\mathcal{O}} \mathcal{O}^{\text {ur }}\right)
$$

We would like to give a map of buildings

$$
\iota: \mathcal{B}\left(G, K^{\mathrm{ur}}\right) \rightarrow \mathcal{B}\left(\mathrm{GL}(V), K^{\mathrm{ur}}\right),
$$

such that $\iota\left(x_{o}\right)$ is the point $[\Lambda]$ in $\mathcal{B}\left(\mathrm{GL}(V), K^{\text {ur }}\right)$ which is given by the $\mathcal{O}^{\text {ur }}$-lattice $\Lambda:=\left(\oplus_{i} \Lambda_{i}\right) \otimes_{\mathcal{O}} \mathcal{O}^{\text {ur }}$. By definition, this is the point $[\Lambda]:=\left(\left\{\pi^{n} \Lambda\right\}_{n \in \mathbb{Z}}, c_{\Lambda}\right)$, with $c_{\Lambda}\left(\pi^{n} \Lambda\right)=n$.

Proposition 1.2.3. We assume that $G$ is split and let $x_{o}, \rho: G \rightarrow \mathrm{GL}(V)$ be as above. There exists a $\operatorname{Gal}\left(K^{\mathrm{ur}} / K\right)$ - and $G\left(K^{\mathrm{ur}}\right)$-equivariant toral map

$$
\iota: \mathcal{B}\left(G, K^{\mathrm{ur}}\right) \rightarrow \mathcal{B}\left(\mathrm{GL}(V), K^{\mathrm{ur}}\right),
$$

such that $\iota\left(x_{o}\right)$ is the point which corresponds to $\Lambda \otimes_{\mathcal{O}} \mathcal{O}^{\text {ur }}=\left(\oplus_{i} \Lambda_{i}\right) \otimes \mathcal{O}^{\text {ur }}$ as described above. Suppose in addition that $\rho: G \rightarrow \mathrm{GL}(V)$ is faithful. Then $\iota$ is an isometric embedding and is the unique $\mathrm{Gal}\left(K^{\mathrm{ur}} / K\right)$ - and $G\left(K^{\mathrm{ur}}\right)$-equivariant toral embedding with $\iota\left(x_{o}\right)$ as above. The map $\iota$ gives by restriction a $G(K)$-equivariant toral isometric embedding $\iota: \mathcal{B}(G, K) \rightarrow \mathcal{B}(\mathrm{GL}(V), K)$. 
Proof. By [48, Theorem 2.1.8] and its proof, there is a canonical $G\left(K^{\mathrm{ur}}\right)$ - and $\operatorname{Gal}\left(K^{\mathrm{ur}} / K\right)$-equivariant toral map $a_{i}: \mathcal{B}\left(G, K^{\mathrm{ur}}\right) \rightarrow \mathcal{B}\left(G_{i}, K^{\mathrm{ur}}\right)$. (When $K=$ $k((\pi))$, even though $K$ is not perfect, we see using the separability assumption, that the proof of [48, Theorem 2.1.8] extends.) Under this, the image of the hyperspecial $x_{o} \in \mathcal{B}(G, K)$ is a hyperspecial $x_{o, i} \in \mathcal{B}\left(G_{i}, K\right)$. Denote by $G_{i, \mathcal{O}}$ the reductive group scheme over $\mathcal{O}$ that corresponds to $x_{o, i}$. Using [11, (1.7.6)], we see that $a_{i}$ extends to a group scheme homomorphism $a_{i, \mathcal{O}}: G_{\mathcal{O}} \rightarrow G_{i, \mathcal{O}}$. Recall that $\rho_{i}\left(G_{\mathcal{O}}\left(\mathcal{O}^{\text {ur }}\right)\right) \subset \mathrm{GL}\left(\Lambda_{i} \otimes_{\mathcal{O}} \mathcal{O}^{\text {ur }}\right)$.

Lemma 1.2.5. We have $G_{i, \mathcal{O}}\left(\mathcal{O}^{\text {ur }}\right) \subset \mathrm{GL}\left(\Lambda_{i} \otimes_{\mathcal{O}} \mathcal{O}^{\text {ur }}\right)$ and $G_{i} \hookrightarrow \mathrm{GL}\left(V_{i}\right)$ extends to a group scheme homomorphism $G_{i, \mathcal{O}} \rightarrow \mathrm{GL}\left(\Lambda_{i}\right)$.

Proof. Note that $a_{i, \mathcal{O}}\left(\mathcal{O}^{\text {ur }}\right): G_{\mathcal{O}}\left(\mathcal{O}^{\text {ur }}\right) \rightarrow G_{i, \mathcal{O}}\left(\mathcal{O}^{\text {ur }}\right)$ is not always surjective. For every root subgroup $U_{i}$ of $G_{i}$, there is a root subgroup $U$ of $G$ such that $a_{i, \mathcal{O} \mid U}: U \rightarrow$ $U_{i}$ is an isomorphism; this extends to an isomorphism of corresponding integral root subgroups $\mathcal{U}_{i}$ and $\mathcal{U}$. Therefore, the $\mathcal{O}^{\text {ur }}$-valued points of each root subgroup $\mathcal{U}_{i}$ of $G_{i, \mathcal{O}}$ belong to the image of $a_{i, \mathcal{O}}\left(\mathcal{O}^{\text {ur }}\right)$ and therefore lie in $\operatorname{GL}\left(\Lambda_{i} \otimes_{\mathcal{O}} \mathcal{O}^{\text {ur }}\right)$. Now let $T$ be a maximal split torus of $G$ such that $x_{o}$ is in the apartment of $T$. The image $T_{i}$ of $T$ under $a_{i}$ is a maximal spit torus of $G_{i}$ and $x_{o, i}$ is in the apartment of $T_{i}$. Suppose that $\mathcal{T} \simeq \mathbb{G}_{m, \mathcal{O}}^{r} \subset G_{\mathcal{O}}$, resp. $\mathcal{T}_{i} \simeq \mathbb{G}_{m, \mathcal{O}}^{r_{i}} \subset G_{i, \mathcal{O}}$, are the Néron models of $T$, resp. $T_{i}$. Then $a_{i, \mathcal{O}}$ restricts to $\mathcal{T} \rightarrow \mathcal{T}_{i}$. By our assumption, $\rho_{i}$ gives a group scheme homomorphism $\mathcal{T} \rightarrow \mathrm{GL}\left(\Lambda_{i}\right)$, which amounts to a grading of $\Lambda_{i}$ by the character group $\mathbb{X} \bullet(T)=\mathbb{X} \bullet(\mathcal{T}) \simeq \mathbb{Z}^{r}$ of $\mathcal{T}$. Since the representation $G \rightarrow \mathrm{GL}\left(V_{i}\right)$ factors through $a_{i}$, the non-zero graded pieces of $\Lambda_{i}$ appear only for characters in the subgroup $\mathbb{X}^{\bullet}\left(\mathcal{T}_{i}\right) \subset \mathbb{X} \bullet(\mathcal{T})$. This shows that there is $\mathcal{T}_{i} \rightarrow \operatorname{GL}\left(\Lambda_{i}\right)$ such that $\mathcal{T} \rightarrow \mathrm{GL}\left(\Lambda_{i}\right)$ is the composition $\mathcal{T} \rightarrow \mathcal{T}_{i} \rightarrow \mathrm{GL}\left(\Lambda_{i}\right)$. Hence, $\mathcal{T}_{i}\left(\mathcal{O}^{\text {ur }}\right) \subset$ $\operatorname{GL}\left(\Lambda_{i} \otimes_{\mathcal{O}} \mathcal{O}^{\text {ur }}\right)$. Since $G_{i, \mathcal{O}}\left(\mathcal{O}^{\text {ur }}\right)$ is generated by $\mathcal{U}_{i}\left(\mathcal{O}^{\text {ur }}\right)$ (for all root subgroups) and $\mathcal{T}_{i}\left(\mathcal{O}^{\text {ur }}\right)$ (see e.g. [11, 4.6]), we conclude that $G_{i, \mathcal{O}}\left(\mathcal{O}^{\text {ur }}\right) \subset \mathrm{GL}\left(\Lambda_{i} \otimes_{\mathcal{O}} \mathcal{O}^{\text {ur }}\right)$. The second statement then follows from [11, (1.7.6)].

We will now use [48, Theorem 2.2.9] to produce a $G_{i}\left(K^{\mathrm{ur}}\right)$ - and $\operatorname{Gal}\left(K^{\mathrm{ur}} / K\right)$ equivariant toral isometric embedding of buildings $\mathcal{B}\left(G_{i}, K^{\mathrm{ur}}\right) \hookrightarrow \mathcal{B}\left(\mathrm{GL}\left(V_{i}\right), K^{\mathrm{ur}}\right)$ that maps $x_{o, i}$ to $y_{i}=\left[\Lambda_{i} \otimes_{\mathcal{O}} \mathcal{O}^{\text {ur }}\right]$. The point $y_{i}$ in the building of $\operatorname{GL}\left(V_{i}\right)$ satisfies the conditions (TOR), (STAB) and (CENT) of loc. cit.: We can easily check (TOR); (STAB) then follows from loc. cit. Prop. 2.5.2, since both the groups are split. For the same reason, (CENT) trivially follows from (STAB). By [48, Theorem 2.2.9, Prop. 2.2.10] it then follows that there exists a unique $G_{i}(K)$ equivariant toral isometric embedding of buildings $\mathcal{B}\left(G_{i}, K^{\mathrm{ur}}\right) \hookrightarrow \mathcal{B}\left(\mathrm{GL}\left(V_{i}\right), K^{\mathrm{ur}}\right)$ that maps $x_{o, i}$ to $y_{i}$. This map is also $\operatorname{Gal}\left(K^{\mathrm{ur}} / K\right)$-equivariant, since the image $y_{i}$ is fixed by $\operatorname{Gal}\left(K^{\mathrm{ur}} / K\right)$. By composing we now obtain a corresponding $G\left(K^{\mathrm{ur}}\right)$ and $\operatorname{Gal}\left(K^{\mathrm{ur}} / K\right)$-equivariant toral map $\iota_{i}: \mathcal{B}\left(G, K^{\mathrm{ur}}\right) \rightarrow \mathcal{B}\left(\mathrm{GL}\left(V_{i}\right), K^{\mathrm{ur}}\right)$. By combining the maps above, we obtain a $G\left(K^{\mathrm{ur}}\right)$ - and $\operatorname{Gal}\left(K^{\mathrm{ur}} / K\right)$-equivariant toral map

$$
\iota: \mathcal{B}\left(G, K^{\mathrm{ur}}\right) \stackrel{\left(\iota_{i}\right)_{i}}{\longrightarrow} \prod_{i} \mathcal{B}\left(\mathrm{GL}\left(V_{i}\right), K^{\mathrm{ur}}\right)=\mathcal{B}\left(\prod_{i} \mathrm{GL}\left(V_{i}\right), K^{\mathrm{ur}}\right) \subset \mathcal{B}\left(\mathrm{GL}(V), K^{\mathrm{ur}}\right) .
$$

See [48, Prop. 2.1.6] for the equality in the middle, above. The last embedding in the display is obtained as follows: Since $\prod_{i} \mathrm{GL}\left(V_{i}\right)$ is a Levi subgroup of $\mathrm{GL}(V)$, we can apply [48, Prop. 2.1.5] and obtain an embedding which sends the point corresponding to $\left(\left[\Lambda_{i} \otimes_{\mathcal{O}} \mathcal{O}^{\text {ur }}\right]\right)_{i}$ to the point given by the $\mathcal{O}^{\text {ur }}$-lattice $\oplus_{i}\left(\Lambda_{i} \otimes_{\mathcal{O}}\right.$ 
$\left.\mathcal{O}^{\text {ur }}\right) \subset V \otimes_{K} K^{\text {ur }}$. If $\rho$ is faithful, then $\iota$ is injective and so it gives an embedding. The uniqueness then follows from [48, Prop. 2.2.10].

Remark 1.2.7. a) When $\rho$ is faithful, the embedding $\iota$ as above can also be obtained directly from the "descent" of root valuation data of [10, 9.1.19 (c)] by using that $\rho$ maps the hyperspecial subgroup $G_{\mathcal{O}}\left(\mathcal{O}^{\text {ur }}\right)$ to $\operatorname{GL}\left(\oplus_{i}\left(\Lambda_{i} \otimes_{\mathcal{O}} \mathcal{O}^{\text {ur }}\right)\right)$.

b) For any $t \in \mathbb{R}$, we also have a $G\left(K^{\text {ur }}\right)$-equivariant toral map $t+\iota: \mathcal{B}\left(G, K^{\text {ur }}\right) \rightarrow$ $\mathcal{B}\left(\mathrm{GL}(V), K^{\text {ur }}\right)$ determined by $(t+\iota)(x)=\left(\left\{\pi^{n} \Lambda\right\}_{n \in \mathbb{Z}}, c_{\Lambda}+t\right)$. This map is also $\operatorname{Gal}\left(K^{\mathrm{ur}} / K\right)$-equivariant. For every $x \in \mathcal{B}\left(G, K^{\mathrm{ur}}\right),(t+\iota)(x)$ and $\iota(x)$ have the same stabilizer in $\mathrm{GL}\left(V \otimes_{K} K^{\mathrm{ur}}\right)$.

c) More generally, suppose that, for each $i$, we have a pair $\left(\Lambda_{i}, t_{i}\right)$ of a $\mathcal{O}$-lattice $\Lambda_{i} \subset V_{i}$ and a real number $t_{i} \in \mathbb{R}$ which determine the point $\left(\left\{\pi^{n} \Lambda_{i}\right\}_{n \in \mathbb{Z}}, c_{\Lambda_{i}}+t_{i}\right)$ in the building $\mathcal{B}\left(\mathrm{GL}\left(V_{i}\right), K\right)$. Suppose also that $\rho_{i}\left(G_{\mathcal{O}}\left(\mathcal{O}^{\text {ur }}\right)\right) \subset \operatorname{GL}\left(\Lambda_{i} \otimes_{\mathcal{O}} \mathcal{O}^{\text {ur }}\right)$, for each $i$. Then the proof of Proposition 1.2.3 extends to give a $\operatorname{Gal}\left(K^{\mathrm{ur}} / K\right)$ - and $G\left(K^{\mathrm{ur}}\right)$-equivariant toral map

$$
\iota: \mathcal{B}\left(G, K^{\mathrm{ur}}\right) \rightarrow \mathcal{B}\left(\mathrm{GL}(V), K^{\mathrm{ur}}\right)
$$

such that $\iota\left(x_{o}\right)$ is the image of $\left(\left\{\pi^{n}\left(\Lambda_{i} \otimes \mathcal{O} \mathcal{O}^{\text {ur }}\right)\right\}_{n \in \mathbb{Z}}, c_{\Lambda_{i}}+t_{i}\right)_{i}$ under the Levi embedding $\prod_{i} \mathcal{B}\left(\mathrm{GL}\left(V_{i}\right), K^{\mathrm{ur}}\right)=\mathcal{B}\left(\prod_{i} \mathrm{GL}\left(V_{i}\right), K^{\mathrm{ur}}\right) \subset \mathcal{B}\left(\mathrm{GL}(V), K^{\mathrm{ur}}\right)$. If $\rho$ is faithful, this map is an isometric embedding and is unique. Note that this $\iota\left(x_{o}\right)$ is not always hyperspecial. For example, if $K=\mathbb{Q}_{p}$ and $\rho: G=\mathbb{G}_{m}^{2} \hookrightarrow \mathrm{GL}_{2}$ is the embedding of the diagonal torus, all points of the corresponding apartment can appear as $\iota\left(x_{o}\right)$. Indeed, all points of the apartment are translations of $\left[\mathbb{Z}_{p} e_{1} \oplus \mathbb{Z}_{p} e_{2}\right]$ by some $\left(t_{1}, t_{2}\right) \in \mathbb{R}^{2}$.

d) Observe that in Proposition 1.2.3 and also in (c) above, the map of buildings $\iota$ factors through a "Levi embedding", with the Levi subgroup determined by a decomposition of the representation $V$ as a direct sum of irreducibles; we use this in the proof of Proposition 1.3.3. In general, there are equivariant maps that do not factor this way.

1.2.9. For the rest of this section, unless we explicitly discuss the case $K=k((\pi))$, we will assume that $\operatorname{char}(K)=0$.

Let $T \subset G$ be a maximal torus. We will say that $\rho$ is minuscule if $\rho \otimes_{K} \bar{K}$ is isomorphic to a direct sum of irreducible representations which are minuscule in the sense that the weights of the corresponding representation of $\operatorname{Lie}\left(G_{\bar{K}}^{\text {der }}\right)$ on $V_{\bar{K}}$ for the Cartan subalgebra $\operatorname{Lie}\left(T_{\bar{K}}^{\mathrm{der}}\right)$ are conjugate under the Weyl group. (See [7], Ch. VI, $\S 1$, ex. 24, $\S 4$, ex. 15]). This notion is independent of the choice of $T$. When $G=\mathrm{SL}_{2}$ the irreducible minuscule representations are the standard and the trivial representation.

Proposition 1.2.10. Suppose that $G$ is split over $K$ and that $\rho: G \rightarrow \mathrm{GL}(V)$ is minuscule and irreducible. Assume that $\Lambda, \Lambda^{\prime}$ are two $\mathcal{O}$-lattices in $V$ such that $\rho\left(G_{\mathcal{O}}\left(\mathcal{O}^{\text {ur }}\right)\right) \subset \operatorname{GL}\left(\Lambda \otimes_{\mathcal{O}} \mathcal{O}^{\text {ur }}\right) \cap \mathrm{GL}\left(\Lambda^{\prime} \otimes_{\mathcal{O}} \mathcal{O}^{\text {ur }}\right)$, the intersection taking place in $\mathrm{GL}\left(V \otimes_{K} K^{\mathrm{ur}}\right)$. Then $\Lambda$ and $\Lambda^{\prime}$ are in the same homothety class, i.e. $\Lambda^{\prime}=\pi^{n} \Lambda$, for some $n \in \mathbb{Z}$.

Proof. By [11, (1.7.6)], our assumption implies that $\rho$ extends to group scheme homomorphisms $\rho_{\mathcal{O}}: G_{\mathcal{O}} \rightarrow \mathrm{GL}(\Lambda), \rho_{\mathcal{O}}^{\prime}: G_{\mathcal{O}} \rightarrow \mathrm{GL}\left(\Lambda^{\prime}\right)$. Let $T \subset G$ be a maximal split torus such that $x_{o}$ is in the apartment of $T$, and let $\mathcal{T} \subset G_{\mathcal{O}}$ be the Néron model of $T$. The torus $\mathcal{T}$ acts on $\Lambda$, and we can decompose $\Lambda$ as direct sum of weight spaces $\Lambda=\oplus_{\lambda \in W(\rho)} \Lambda_{\lambda}$. Since $\rho$ is minuscule, the set of weights $W(\rho) \subset \mathbb{X}^{\bullet}(T)$ is 
an orbit $W \cdot \lambda_{0}$ of a single highest weight $\lambda_{0}$ under the Weyl group and all the spaces $V_{\lambda}$ are one dimensional (7, Ch. VIII, $\left.\S 7,3\right]$ ). In particular, it follows that $\Lambda \otimes_{\mathcal{O}} k$ is an irreducible $G \otimes_{\mathcal{O}} k$-representation [41, II 2.15].

After replacing $\Lambda^{\prime}$ by a scalar multiple, we may assume that $\Lambda^{\prime} \subset \Lambda$, and that if $\bar{\Lambda}^{\prime} \subset \Lambda \otimes_{\mathcal{O}} k$ denotes the image of $\Lambda^{\prime}$ in $\Lambda \otimes_{\mathcal{O}} k$, then $\bar{\Lambda}^{\prime} \neq\{0\}$. Then $\bar{\Lambda}^{\prime} \subset \Lambda \otimes_{\mathcal{O}} k$ is a non-zero $G \otimes_{\mathcal{O}} k$-subrepresentation. As $\Lambda \otimes_{\mathcal{O}} k$ is irreducible this implies $\bar{\Lambda}^{\prime}=\bar{\Lambda}$, and so $\Lambda^{\prime}=\Lambda$, as desired.

Corollary 1.2.11. Assume that, in addition to the above assumptions, $\rho$ is faithful. If $\iota$ and $\iota^{\prime}$ are $G\left(K^{\mathrm{ur}}\right)$-equivariant toral embeddings $\mathcal{B}\left(G, K^{\mathrm{ur}}\right) \rightarrow \mathcal{B}\left(\mathrm{GL}(V), K^{\mathrm{ur}}\right)$, then there is $t \in \mathbb{R}$ such that $\iota^{\prime}=t+\iota$.

Proof. By [48, Prop. 2.2.10], such $\iota, \iota^{\prime}$ are determined by the points $\iota\left(x_{o}\right), \iota^{\prime}\left(x_{o}\right)$ in $\mathcal{B}\left(\mathrm{GL}(V), K^{\mathrm{ur}}\right)$. Their stabilizers subgroups both have to contain $\rho\left(G_{\mathcal{O}}\left(\mathcal{O}^{\text {ur }}\right)\right)$ and so by Proposition 1.2 .10 they both have to be hyperspecial. Since such hyperspecial points are determined up to translation by a real number by their stabilizer subgroups, Proposition 1.2.10 implies the result.

1.2.12. We continue to assume that $G$ is split over $K$ and that $\rho: G \rightarrow \operatorname{GL}(V)$ is a $K$-representation.

Denote by $H$ the split Chevalley form of $G$ over $\mathbb{Z}_{p}$; fix a pinning $(T, B, \underline{e})=$ $\left(T_{H}, B_{H}, \underline{e}\right)$ of $H$ over $\mathbb{Z}_{p}$ and a corresponding hyperspecial vertex $x_{o}$ of the building $\mathcal{B}\left(H, \mathbb{Q}_{p}\right)$ whose stabilizer is $H\left(\mathbb{Z}_{p}\right)$. Choose an isomorphism $G \simeq H \otimes_{\mathbb{Z}_{p}} K$, then we can take $G_{\mathcal{O}}=H \otimes_{\mathbb{Z}_{p}} \mathcal{O}$. Recall that if $K^{\prime}$ is any $p$-adic local field extension of $K$, there is a canonical embedding $\mathcal{B}\left(H, \mathbb{Q}_{p}\right) \hookrightarrow \mathcal{B}\left(H, K^{\prime}\right)$ and so we can also think of $x_{o}$ as a hyperspecial vertex of $\mathcal{B}\left(G, K^{\prime}\right)$ for all such $K^{\prime}$.

Let $V=\oplus_{i} V_{i}, \rho=\oplus_{i} \rho_{i}$, with $V_{i}=V\left(\lambda_{i}\right) \otimes_{\mathbb{Q}_{p}} K, V\left(\lambda_{i}\right)$ an irreducible Weyl module of highest weight $\lambda_{i}$ (for our choice of $T, B$ ) over $\mathbb{Q}_{p}$; fix a highest weight vector $v_{i}=v_{\lambda_{i}}$ in $V\left(\lambda_{i}\right)$ and consider the $\mathbb{Z}_{p}$-lattice $\Lambda_{i} \subset V\left(\lambda_{i}\right)$ given as $\Lambda_{i}=\mathfrak{U}_{H}^{-} \cdot v_{i}$ where $\mathfrak{U}_{H}^{-}$is the subalgebra $\mathfrak{U}_{H}$ of the universal enveloping algebra of $H$ over $\mathbb{Z}_{p}$ generated by the negative root spaces acting on $V\left(\lambda_{i}\right)$. This gives $\rho_{i}: H \rightarrow \operatorname{GL}\left(\Lambda_{i}\right)$ (cf. 41]) and we can see that the assumptions of Proposition 1.2.3 are satisfied for the choice of lattices $\Lambda_{i} \otimes_{\mathbb{Z}_{p}} \mathcal{O} \subset V_{i}=V\left(\lambda_{i}\right) \otimes_{\mathbb{Q}_{p}} K$. Hence, we have

$$
\iota: \mathcal{B}\left(G, K^{\mathrm{ur}}\right) \rightarrow \mathcal{B}\left(\mathrm{GL}(V), K^{\mathrm{ur}}\right),
$$

such that $\iota\left(x_{o}\right)$ is the point which corresponds to $\Lambda \otimes_{\mathbb{Z}_{p}} \mathcal{O}^{\text {ur }}=\left(\oplus_{i} \Lambda_{i}\right) \otimes \mathcal{O}^{\text {ur }}$ as described above. More generally, we will also consider maps $\iota$ that also depend on the choice of a collection of $t_{i} \in \mathbb{R}$, as in Remark 1.2.7 (c). The choice above corresponds to $t_{i}=0$. If $\rho$ is faithful, $\iota$ is an embedding.

1.2.14. We now allow $G$ to be non-split; however, we always suppose that $G$ splits over a tamely ramified Galois extension $\tilde{K} / K$ with Galois group $\Gamma=\operatorname{Gal}(\tilde{K} / K)$. We allow $\tilde{K} / K$ to be infinite, but we assume that the inertia subgroup of $\Gamma$ is finite.

Choose an isomorphism $\psi: G \otimes_{K} \tilde{K} \stackrel{\sim}{\rightarrow} H \otimes_{\mathbb{Z}_{p}} \tilde{K}$ which identifies $G(\tilde{K})$ and $H(\tilde{K})$ and write $G(K)=H(\tilde{K})^{\Gamma}$ where the action of $\Gamma$ is given by $\gamma \cdot \tilde{h}=c(\gamma) \cdot \gamma(\tilde{h})$ with $c: \Gamma \rightarrow \operatorname{Aut}(H)(\tilde{K})$ the cocycle $c(\gamma)=\psi \cdot \gamma(\psi)^{-1}$. The cocycle $c$ represents the class of the form $G$ of $H$ in $\mathrm{H}^{1}(\Gamma, \operatorname{Aut}(H)(\tilde{K}))$. Our choice of pinning of $H$ allows us to write $\operatorname{Aut}(H)(\tilde{K})$ as a semi-direct product

$$
\operatorname{Aut}(H)(\tilde{K})=H^{\text {ad }}(\tilde{K}) \rtimes \Xi
$$


where $\Xi=\Xi_{H}$ is the group of Dynkin diagram automorphisms (which is then identified with the subgroup of automorphisms of $H$ that respect the chosen pinning).

Under the assumption of tameness, by work of Rousseau or 60, the canonical map $\mathcal{B}(G, K) \hookrightarrow \mathcal{B}(G, \tilde{K})$ gives identifications $\mathcal{B}(G, K)=\mathcal{B}(G, \tilde{K})^{\Gamma}=\mathcal{B}(H, \tilde{K})^{\Gamma}$; the action of $\Gamma$ on $\mathcal{B}(H, \tilde{K})$ is induced by the action of $\Gamma$ on $H(\tilde{K})$ given above.

1.2.15. We now assume that $G$ is as above and consider a representation $\rho$ : $G \rightarrow \mathrm{GL}(V)$ (i.e. defined over $K)$. In what follows, assuming in addition that $\rho$ is minuscule, we will construct a certain $G\left(K^{\mathrm{ur}}\right)$ - and $\mathrm{Gal}\left(K^{\mathrm{ur}} / K\right)$-equivariant toral map

$$
\iota: \mathcal{B}\left(G, K^{\mathrm{ur}}\right) \rightarrow \mathcal{B}\left(\mathrm{GL}(V), K^{\mathrm{ur}}\right)
$$

which also restricts to give a map $\iota: \mathcal{B}(G, K) \rightarrow \mathcal{B}(\mathrm{GL}(V), K)$.

Assume first that $\rho: G \rightarrow \mathrm{GL}(V)$ is irreducible over $K$; we do not assume that $\rho$ is faithful. We follow the arguments of [69] or [66]. (See, for example, the proof of Theorem 7.6 in [69]). Let

$$
D^{0}=\left\{\varphi \in \operatorname{End}_{K}(V) \mid \varphi \cdot \rho(g)=\rho(g) \cdot \varphi, \forall g \in G(\tilde{K})\right\}
$$

be the centralizer algebra of $\rho$, which is a division $K$-algebra. Then $V$ is a (right) module for the opposite $K$-algebra $D=\left(D^{0}\right)^{\text {opp }}$.

The Galois group $\Gamma$ acts naturally on the set of dominant weights of $G$ as described in [69, 3.1]. For a dominant weight $\lambda$, we denote by $V_{\lambda, \tilde{K}}$ the $\tilde{K}$-subspace of $V \otimes_{K} \tilde{K}$ generated by all simple submodules of highest weight $\lambda$. Let $\lambda_{1}, \ldots, \lambda_{r}$ be the dominant weights $\lambda$ for which $V_{\lambda, \tilde{K}} \neq 0$. This set is $\Gamma$-stable, and we have

$$
V \otimes_{K} \tilde{K}=\oplus_{i=1}^{r} V_{\lambda_{i}, \tilde{K}} .
$$

The $\Gamma$-action on $V \otimes_{K} \tilde{K}$ induces a transitive action on the set of summands $V_{\lambda_{i}, \tilde{K}}$, which coincides with the one induced by the action of $\Gamma$ on $\left\{\lambda_{i}\right\}_{i}$. As in loc. cit., we have

$$
V_{\lambda_{i}, \tilde{K}} \simeq V\left(\lambda_{i}\right)^{\oplus d} \otimes_{\mathbb{Q}_{p}} \tilde{K}
$$

where $d$ is an integer not depending on $i$. Denote by $\Gamma_{1} \subset \Gamma$ the stabilizer of $\lambda_{1}$; let $K_{1}$ be the corresponding field $K \subset K_{1} \subset \tilde{K}$ and set $V_{1}=V_{\lambda_{1}, \tilde{K}}$. The center of $D$ can be identified with $K_{1}$ and then $V$ becomes a $K_{1}$-vector space; the epimorphism $V \otimes_{K} \tilde{K} \rightarrow V \otimes_{K_{1}} \tilde{K}$ gives an isomorphism $V \otimes_{K_{1}} \tilde{K} \simeq V_{1}$. We obtain a $K_{1^{-}}$ representation

$$
\bar{\rho}_{1}: G_{K_{1}} \rightarrow \mathrm{GL}(V)_{D}
$$

which is absolutely irreducible and is such that $\bar{\rho}_{1} \otimes_{K_{1}} \tilde{K}$ is identified with the Weyl module representation $\rho_{1}: G_{\tilde{K}} \cong H_{\tilde{K}} \rightarrow \operatorname{GL}\left(V\left(\lambda_{1}\right)_{\tilde{K}}\right)$. As in loc. cit., the original $K$-representation $\rho: G \rightarrow \operatorname{GL}(V)$ can be obtained from $\bar{\rho}_{1}$ by applying restriction of scalars twice:

$$
\rho=\operatorname{Res}_{K_{1} / K}\left(\operatorname{Res}_{D / K_{1}} \cdot \bar{\rho}_{1}\right) .
$$

Here, $\operatorname{Res}_{D / K_{1}}: \mathrm{GL}(V)_{D} \hookrightarrow \mathrm{GL}_{K_{1}}(V)$ is given by forgetting the $D$-module structure, and $\operatorname{Res}_{K_{1} / K}: \mathrm{GL}_{K_{1}}(V) \hookrightarrow \mathrm{GL}(V)$ by forgetting the $K_{1}$-module structure. More precisely, $\rho$ is the composition of

$$
G \rightarrow \operatorname{Res}_{K_{1} / K}\left(G_{K_{1}}\right) \stackrel{\operatorname{Res}_{K_{1} / K}\left(\bar{\rho}_{1}\right)}{\longrightarrow} \operatorname{Res}_{K_{1} / K}\left(\mathrm{GL}(V)_{D}\right)
$$


with

$$
\operatorname{Res}_{K_{1} / K}\left(\mathrm{GL}(V)_{D}\right) \rightarrow \operatorname{Res}_{K_{1} / K}\left(\mathrm{GL}(V)_{K_{1}}\right) \rightarrow \mathrm{GL}(V) .
$$

In fact, $\bar{\rho}_{1}: G_{K_{1}} \rightarrow \operatorname{GL}(V)_{D}$ is a $K_{1}$-form of the Weyl module $\rho_{1}$ as follows: The group $\Gamma_{1}$ acts on $G(\tilde{K})=H(\tilde{K})$ with the action given by twisting via the cocycle $c_{\mid \Gamma_{1}}: \Gamma_{1} \rightarrow \operatorname{Aut}(H)(\tilde{K})$. Denote by $J_{1}(\tilde{K})$ the subgroup of $\operatorname{Aut}(H)(\tilde{K})$ generated by $H^{\text {ad }}(\tilde{K})$ together with $c(\gamma)$ for $\gamma \in \Gamma_{1}$. Since $\lambda_{1}$ is $\Gamma_{1}$-invariant, for every $a \in J_{1}(\tilde{K})$, the representation $\rho_{1} \circ a$ is again irreducible of highest weight $\lambda_{1}$, and so there is $\theta(a) \in \operatorname{PGL}\left(V\left(\lambda_{1}\right) \otimes \tilde{K}\right)$ such that $\rho_{1} \circ a=\theta(a) \circ \rho_{1}$; by Schur's lemma, $\theta(a)$ is uniquely determined and hence it gives a homomorphism

$$
\theta: J_{1}(\tilde{K}) \rightarrow \operatorname{PGL}\left(V\left(\lambda_{1}\right) \otimes \tilde{K}\right) .
$$

As in the proof of [69, Theorem 3.3], the cocycle

$$
c^{\prime}:=\theta \cdot c: \Gamma_{1} \rightarrow \operatorname{PGL}\left(V\left(\lambda_{1}\right) \otimes \tilde{K}\right)
$$

defines the $K_{1}$-form $\operatorname{End}(V)_{D}=\operatorname{End}\left(V\left(\lambda_{1}\right) \otimes \tilde{K}\right)^{\Gamma_{1}}$ of $\operatorname{End}\left(V\left(\lambda_{1}\right)\right)$ and $\rho_{1}: H_{\tilde{K}} \rightarrow$ $\mathrm{GL}\left(V\left(\lambda_{1}\right) \otimes \tilde{K}\right)$ descends to

$$
\bar{\rho}_{1}: G_{K_{1}}=(H \otimes \tilde{K})^{\Gamma_{1}} \rightarrow \mathrm{GL}(V)_{D}=\mathrm{GL}\left(V\left(\lambda_{1}\right) \otimes \tilde{K}\right)^{\Gamma_{1}} .
$$

Here, the $\Gamma_{1}$-fixed points are for the $\Gamma_{1}$-actions given using the cocycles $c$ and $c^{\prime}=\theta \cdot c$.

From here and on we will assume that $\tilde{K}$ contains $K^{\text {ur }}$.

Proposition 1.2.21. Assume that $\rho$, or equivalently that $\rho_{1}$, is minuscule. We equip $\mathcal{B}\left(\mathrm{GL}\left(V\left(\lambda_{1}\right)\right), \tilde{K}\right)$ with the action of $\Gamma_{1}$ induced by the standard action on $\mathrm{GL}\left(V\left(\lambda_{1}\right) \otimes \tilde{K}\right)$ twisted by the cocycle $c^{\prime}$.

Then the $G(\tilde{K})=H(\tilde{K})$-equivariant toral map

$$
\iota_{1}: \mathcal{B}(G, \tilde{K})=\mathcal{B}(H, \tilde{K}) \rightarrow \mathcal{B}\left(\operatorname{GL}\left(V\left(\lambda_{1}\right)\right), \tilde{K}\right)
$$

given as in the split case above is $\Gamma_{1}$-equivariant.

Proof. By the construction of $\iota_{1}$ as a composition

$$
\mathcal{B}(H, \tilde{K}) \rightarrow \mathcal{B}\left(H / \operatorname{ker}\left(\rho_{1}\right), \tilde{K}\right) \rightarrow \mathcal{B}\left(\mathrm{GL}\left(V\left(\lambda_{1}\right)\right), \tilde{K}\right),
$$

we see that after replacing $H$ by $H / \operatorname{ker}\left(\rho_{1}\right)$, and $G_{K_{1}}$ by $G_{K_{1}} / \operatorname{ker}\left(\bar{\rho}_{1}\right)$, we are reduced to considering the situation in which we assume in addition that $\rho_{1}$ is faithful. By [48, there is a $G(\tilde{K})$ - and $\Gamma_{1}$-equivariant toral isometric embedding

$$
\iota_{1}^{L}: \mathcal{B}(G, \tilde{K})=\mathcal{B}(H, \tilde{K}) \rightarrow \mathcal{B}\left(\mathrm{GL}\left(V\left(\lambda_{1}\right)\right), \tilde{K}\right) .
$$

Regard now both $\iota_{1}$ and $\iota_{1}^{L}$ as two $H(\tilde{K})$-equivariant toral isometric maps between the buildings of the split reductive groups $H(\tilde{K})$ and $\operatorname{GL}\left(V\left(\lambda_{1}\right) \otimes \tilde{K}\right)$ over $\tilde{K}$. By Corollary 1.2.11 we have $\iota_{1}=t+\iota_{1}^{L}$ with $t \in \mathbb{R}=\mathbb{X} \bullet\left(\operatorname{diag}\left(\mathbb{G}_{\mathrm{m}} \tilde{K}\right)\right) \otimes_{\mathbb{Z}} \mathbb{R}$. Notice now that the Galois group $\Gamma_{1}$ acts trivially on $\mathbb{X}_{\bullet}\left(\operatorname{diag}\left(\mathbb{G}_{\mathrm{m}} \tilde{K}\right)\right)$. Since $\iota_{1}^{L}$ is $\Gamma_{1^{-}}$ equivariant this implies that $\iota_{1}$ is also $\Gamma_{1}$-equivariant and this concludes the proof.

1.2.22. We continue with the above notations and assume that $\rho$ is minuscule. Recall $\tilde{K}$ contains $K^{\text {ur }}$; let $I_{1} \subset \Gamma_{1}$ be the inertia subgroup. Using [60] and Prop. 1.2.21 we see that by restricting to $I_{1}$-fixed points, $\iota_{1}$ gives

$$
\iota_{1}: \mathcal{B}\left(G, K_{1}^{\mathrm{ur}}\right) \rightarrow \mathcal{B}\left(\mathrm{GL}(V)_{D}, K_{1}^{\mathrm{ur}}\right) .
$$


The same construction also works for the translations $t+\iota_{1}, t \in \mathbb{R}$. This gives

$$
\mathcal{B}\left(G, K^{\mathrm{ur}}\right) \subset \mathcal{B}\left(G, K_{1}^{\mathrm{ur}}\right) \stackrel{t+\iota_{1}}{\longrightarrow} \mathcal{B}\left(\mathrm{GL}(V)_{D}, K_{1}^{\mathrm{ur}}\right) .
$$

Compose this with the standard equivariant embedding

$$
\mathcal{B}\left(\mathrm{GL}(V)_{D}, K_{1}^{\mathrm{ur}}\right)=\mathcal{B}\left(\operatorname{Res}_{K_{1}^{\mathrm{ur}}} / K^{\mathrm{ur}}\left(\mathrm{GL}(V)_{D}\right), K^{\mathrm{ur}}\right) \rightarrow \mathcal{B}\left(\mathrm{GL}(V), K^{\mathrm{ur}}\right)
$$

given by sending $\mathcal{O}_{D}$-lattices in $V$ to the corresponding $\mathcal{O}$-lattices in the $K$-vector space $V$ (by restriction of structure from $\mathcal{O}_{D}$ to $\mathcal{O}$ ). This composition gives a $G\left(K^{\mathrm{ur}}\right)$-equivariant toral map

$$
\iota: \mathcal{B}\left(G, K^{\mathrm{ur}}\right) \rightarrow \mathcal{B}\left(\mathrm{GL}(V), K^{\mathrm{ur}}\right),
$$

which is also $\operatorname{Gal}\left(K^{\mathrm{ur}} / K\right)$-equivariant as desired. This concludes the construction of $\iota$ when $\rho$ is minuscule and irreducible over $K$.

1.2.25. In general, if $\rho: G \rightarrow \mathrm{GL}(V)$ is a minuscule $K$-representation, write it as a direct sum of $K$-irreducible representations $\rho_{j}: G \rightarrow \mathrm{GL}\left(V_{j}\right)$ and then proceed to give a $G(K)$-equivariant toral map $\iota: \mathcal{B}\left(G, K^{\text {ur }}\right) \rightarrow \mathcal{B}\left(\mathrm{GL}(V), K^{\text {ur }}\right)$ by combining $\iota_{j}: \mathcal{B}\left(G, K^{\text {ur }}\right) \rightarrow \mathcal{B}\left(\mathrm{GL}\left(V_{j}\right), K^{\text {ur }}\right)$ given above with the Levi canonical embedding as in (1.2.2). If $\rho$ is faithful, the map $\iota$ is injective. Hence, in this case, we obtain a $G\left(K^{\text {ur }}\right)$-equivariant toral embedding of buildings

$$
\iota: \mathcal{B}\left(G, K^{\mathrm{ur}}\right) \rightarrow \mathcal{B}\left(\mathrm{GL}(V), K^{\mathrm{ur}}\right)
$$

which is also $\operatorname{Gal}\left(K^{\text {ur }} / K\right)$-equivariant as desired.

1.2.27. Consider now the case $K=k((\pi))$. Suppose we have a reductive group $G$ over $K$ which splits over a tamely ramified extension $\tilde{K} / K$ and a representation $\rho: G \rightarrow \mathrm{GL}(V)$. Assume that $\rho$ is written as a direct sum of $G$-representations which are obtained by restriction of scalars as in (1.2.17) of representations $\bar{\rho}_{1}$ given as twisted Weyl modules for minuscule dominant weights $\lambda$. Here we assume that the twist is also given in the same way as $\bar{\rho}_{1}$ is given in the characteristic 0 case of 1.2.15. (Note that in this case, $G$-representations are not in general semi-simple modules; however, here we assume such a direct sum decomposition and we are also giving the twisting construction as in 1.2.15 as part of our data. Also recall, a dominant weight $\lambda$ for a Chevalley group is minuscule if there is no other dominant weight $\mu$ with $\mu<\lambda$, where $\leq$ denotes the usual partial ordering of weights. This implies that the Weyl module $V(\lambda)_{k}$ is simple [41, II, 2.15] and that its weights are the Weyl group orbit of $\lambda$.)

Under the above assumptions, we can obtain maps of buildings $\iota_{1}$ as in Proposition 1.2.21, and then $\iota$ as in (1.2.26), by carrying out the same construction as above. (Note that, under our assumptions, $H \rightarrow H / \operatorname{ker}\left(\rho_{1}\right)$ is separable on each root subgroup -for that see also the proof of Proposition 1.3.3 below that reduces this to the case $H=\mathrm{SL}_{2^{-}}$and so we can apply Proposition 1.2.3 as a step in our construction.)

\subsection{Minuscule representations and group schemes.}

1.3.1. We continue to assume that $G$ splits over a tamely ramified extension $\tilde{K}$ of $K$ with Galois group $\Gamma=\operatorname{Gal}(\tilde{K} / K)$. We assume that $\rho: G \hookrightarrow \operatorname{GL}(V)$ is a faithful minuscule representation of $G$ where $V$ is a finite dimensional $K$-vector space. Recall the $G(K)$-embedding

$$
\iota: \mathcal{B}(G, K) \rightarrow \mathcal{B}(\mathrm{GL}(V), K)
$$


constructed in the previous paragraph. This depends on a choice of an isomorphism $\psi: G_{\tilde{K}} \stackrel{\sim}{\rightarrow} H_{\tilde{K}}$ and a hyperspecial vertex $x_{o}$ of $\mathcal{B}(H, K)$ together with choices of, for each $K$-irreducible summand, a lattice $\Lambda_{1}=\mathfrak{U}_{H}^{-} \cdot v_{1}$ given by the highest weight vector $v_{1} \in V\left(\lambda_{1}\right)$ and a grading $c_{\Lambda_{i}}+t_{i}$ of the lattice chain $\left\{\pi^{n} \Lambda_{i}\right\}_{n \in \mathbb{Z}}$ given by $t_{i} \in \mathbb{R}$. The map $\iota$ appears as a restriction of a $\operatorname{Gal}\left(K^{\mathrm{ur}} / K\right)$-equivariant $G\left(K^{\mathrm{ur}}\right)$ embedding $\iota: \mathcal{B}\left(G, K^{\mathrm{ur}}\right) \rightarrow \mathcal{B}\left(\mathrm{GL}(V), K^{\mathrm{ur}}\right)$.

Proposition 1.3.3. For any $x \in \mathcal{B}(G, K)$, $\rho$ extends to a closed immersion

$$
\rho_{x}: \mathcal{G}_{x} \rightarrow \mathcal{G} \mathcal{L}(V)_{\iota(x)}
$$

of group schemes over $\operatorname{Spec}(\mathcal{O})$.

Proof. Let $y=\iota(x)$ and suppose that $\Lambda_{y}^{\bullet}=\left\{\Lambda_{y}^{i}\right\}_{i \in \mathbb{Z}}$ is the periodic chain of $\mathcal{O}$ lattices in $V$ that corresponds to $y$ and is fixed by $\mathrm{GL}(V)_{y}$. Then $G\left(K^{\mathrm{ur}}\right)_{x}=$ $G\left(K^{\mathrm{ur}}\right) \cap \mathrm{GL}\left(V \otimes_{K} K^{\mathrm{ur}}\right)_{y} \hookrightarrow \mathrm{GL}\left(V \otimes_{K} K^{\mathrm{ur}}\right)_{y}=\mathrm{GL}\left(\Lambda_{y}^{\bullet} \otimes_{\mathcal{O}} \mathcal{O}^{\mathrm{ur}}\right)$. Using [11, (1.7.6)], we obtain a group scheme homomorphism

$$
\rho: \mathcal{G}_{x} \rightarrow \mathcal{G L}_{y}
$$

which we would like to show is a closed immersion. Denote by $\mathcal{G}_{x}^{\prime}$ the schematic closure of $G$ in $\mathcal{G} \mathcal{L}_{y}$; this agrees with the scheme theoretic image of $\rho$ above. Notice that $y=\iota(x)$ implies $\mathcal{G}_{x}^{\prime}\left(\mathcal{O}^{\text {ur }}\right)=G\left(K^{\text {ur }}\right) \cap \mathrm{GL}\left(V \otimes_{K} K^{\text {ur }}\right)_{y}=G\left(K^{\text {ur }}\right)_{x}=\mathcal{G}_{x}\left(\mathcal{O}^{\text {ur }}\right)$. Therefore, it is enough to show that the schematic closure $\mathcal{G}_{x}^{\prime}$ of $G \hookrightarrow \operatorname{GL}(V)$ in $\mathcal{G} \mathcal{L}_{y}$ is smooth or equivalently (by the description of $\mathcal{G} \mathcal{L}_{y}$ recalled in 1.1.9), that the schematic closure of $G \hookrightarrow \prod_{i=0}^{r-1} \mathrm{GL}(V)$ (embedded diagonally) in the $\mathcal{O}$-group scheme $\prod_{i=0}^{r-1} \operatorname{GL}\left(\Lambda_{y}^{i}\right)$ is smooth.

1) We first suppose that $G$ is split over $K$. Fix a maximal $K$-split torus $T \simeq \mathbb{G}_{\mathrm{m}}^{r}$ of $G$ such that $x$ belongs to the apartment $A(G, T, K) \subset \mathcal{B}(G, K)$. To start with, we also assume that $G$ is semi-simple, i.e. $G=G^{\text {der }}$. We first assume that $\rho$ is actually irreducible. The torus $T$ acts on $V$ via $\rho$ and we obtain the weight decomposition

$$
V=\oplus_{\lambda \in W(\rho)} V_{\lambda} .
$$

Since $\rho$ is minuscule, the set of weights $W(\rho) \subset \mathbb{X}^{\bullet}(T)$ is an orbit $W \cdot \lambda_{0}$ of a single weight $\lambda_{0}$ under the Weyl group and all the spaces $V_{\lambda}$ are one dimensional ([7, Ch. VIII, $\S 7,3])$. Set $T^{b}=\prod_{\lambda \in W(\rho)} \operatorname{GL}\left(V_{\lambda}\right)$ for the maximal torus of $\operatorname{GL}(V)$ that preserves the grading above. We have $\rho(T) \subset T^{b}$.

For a root $a \in \Phi(G, T)$, we denote by $U_{a}$ the corresponding unipotent subgroup of $G$. Set $G_{a}=\left\langle U_{a}, U_{-a}\right\rangle$ for the subgroup of $G$ generated by $U_{a}$ and $U_{-a}$. This is isomorphic to either $\mathrm{SL}_{2}$ or $\mathrm{PSL}_{2}$. The isomorphism takes the standard unipotent subgroups $U_{ \pm}$of $\mathrm{SL}_{2}$ to $U_{ \pm a} \subset G$. Consider now the restriction $\rho: G_{a} \rightarrow \operatorname{GL}(V)$ and the composition with the central isogeny $\mathrm{SL}_{2} \rightarrow G_{a}$

$$
\rho_{a}: \mathrm{SL}_{2} \rightarrow \mathrm{GL}(V) \text {. }
$$

We claim that this is a minuscule representation of $\mathrm{SL}_{2}$ : Indeed, consider $V$ as a representation of $\operatorname{Lie}\left(G_{a}\right) \simeq s l_{2}$. It decomposes as follows

$$
V=\oplus_{[\lambda]} V_{[\lambda]}=\oplus_{[\lambda]}\left(\oplus_{\lambda^{\prime}=\lambda+k a} V_{\lambda}\right) .
$$

Here $[\lambda]$ runs over all equivalence classes of weights in $W(\rho)$ under: $\lambda^{\prime} \sim \lambda$ if there is $k \in \mathbb{Z}$ with $\lambda^{\prime}-\lambda=k a$. By the general theory (e.g. 7, Ch. VIII, $\S 7,2$, Prop. $3]), V_{[\lambda]}$ are representations of $s l_{2}=\left\langle X_{-a}, H_{a}, X_{a}\right\rangle$ (a standard Chevalley basis) and there are two cases: 
a) $[\lambda]=\{\lambda\}$ has only one element,

b) $[\lambda]$ has two elements and we can then assume it is of the form $\{\lambda, \lambda+a\}$.

In the first case, $V_{[\lambda]}$ is the trivial representation of $s l_{2}$; in the second case, $V_{[\lambda]}$ is isomorphic to the standard representation of $s l_{2}$. Therefore, for each root $a \in \Phi(G, T)$, the composition $\rho_{a}: \mathrm{SL}_{2} \rightarrow \mathrm{GL}(V)$ is a minuscule representation. It now follows that $\rho_{a}$ does not factor through $\mathrm{PSL}_{2}$ and so $G_{a}$ has to be isomorphic to $\mathrm{SL}_{2}$.

By the construction of $\mathcal{G}_{x}$, the schematic closure $\mathcal{T}$ of $T \subset G$ in $\mathcal{G}_{x}$ is smooth and so it acts on $\Lambda_{y}^{i}$ for all indices $i$. Since $\mathcal{T} \simeq \mathbb{G}_{m / \mathcal{O}}^{r}$, we obtain decompositions

$$
\Lambda_{y}^{i}=\oplus_{\lambda \in W(\rho)} \Lambda_{\lambda, y}^{i}
$$

with $\Lambda_{\lambda, y}^{i} \subset V_{\lambda}$ rank $1 \mathcal{O}$-lattices in $V_{\lambda}$. (This implies that the point $y$ lies in the apartment $A\left(\mathrm{GL}(V), T^{b}, K\right) \subset \mathcal{B}(\mathrm{GL}(V), K)$.) We can now use this to reduce to the case that $G$ is $\mathrm{SL}_{2}$. Write $U_{ \pm a}, G_{a} \simeq \mathrm{SL}_{2}$ as before.

We now allow $V$ to be reducible and write $V=\oplus_{j} V_{j}$ where $V_{j}$ are irreducible and minuscule. By the above applied to the irreducible $V_{j}$, we can write

$$
V_{j}=\oplus_{[\lambda]} V_{j,[\lambda]}=\oplus_{[\lambda]}\left(\oplus_{\lambda^{\prime}=\lambda+k a} V_{j, \lambda}\right)
$$

as before. We have

$$
\Lambda_{y}^{i}=\oplus_{j} \oplus_{\lambda \in W\left(\rho_{j}\right)} \Lambda_{j, \lambda, y}^{i}
$$

with $\Lambda_{j, \lambda, y}^{i} \subset V_{j, \lambda}$ rank $1 \mathcal{O}$-lattices in $V_{j, \lambda}$. Here, we also use the construction of $\iota$, see 1.2.25 and 1.2 .3 and also Remark 1.2.7 (d). We can now see that the schematic closures $\mathcal{U}_{ \pm a}$ of $U_{ \pm a}$ in $\prod_{i} \operatorname{GL}\left(\Lambda_{y}^{i}\right)$ are isomorphic to the schematic closures $\mathcal{U}_{ \pm}$, of

$$
U_{ \pm} \subset \mathrm{SL}_{2} \stackrel{\rho}{\longrightarrow} \prod_{j,[\lambda]} \prod_{i} \mathrm{GL}\left(V_{j,[\lambda]}\right)
$$

in the group scheme

$$
\prod_{j,[\lambda]} \prod_{i} \operatorname{GL}\left(\Lambda_{j,[\lambda], y}^{i}\right)
$$

where $\Lambda_{j,[\lambda], y}^{i}=\Lambda_{j, \lambda, y}^{i}$ or $\Lambda_{j, \lambda, y}^{i} \oplus \Lambda_{j, \lambda+a, y}^{i}$ (in cases (a) or (b) respectively). Consider classes [ $\lambda]$ for which the $\mathrm{SL}_{2}$ representation $V_{j,[\lambda]}$ is not trivial, as in (b) above. We choose a basis vector $e_{j, \lambda}$ of $V_{j, \lambda}$ and set $f_{j, \lambda}=X_{a} \cdot e_{j, \lambda}$ which is a generator of $V_{j, \lambda+a}$. The choice of basis $e_{j, \lambda}, f_{j, \lambda}$, of $V_{j,[\lambda]}$ gives an identification of $V_{j,[\lambda]}$ with the standard representation of $\mathrm{SL}_{2}$. We have

$$
\Lambda_{j, \lambda, y}^{i}=\pi^{n_{j,[\lambda], i}} \mathcal{O} \cdot e_{j, \lambda}, \quad \Lambda_{j, \lambda+a, y}^{i}=\pi^{m_{j,[\lambda], i}} \mathcal{O} \cdot f_{j, \lambda},
$$

for some $m_{j,[\lambda], i}, n_{j,[\lambda], i} \in \mathbb{Z}$, and so under this identification, the lattices $\Lambda_{j,[\lambda], y}^{i} \subset$ $V_{j,[\lambda]}$, for all $i$, are in the same apartment for $\mathrm{GL}\left(V_{j,[\lambda]}\right)$, namely the standard apartment for the chosen basis. It now follows from [12, 3.6, and $3.9(2)]$ that the schematic closures of $U_{ \pm}$in $\prod_{j,[\lambda]} \prod_{i} \operatorname{GL}\left(\Lambda_{j,[\lambda], y}^{i}\right)$ are smooth. Hence, the same is true for the schematic closures $\mathcal{U}_{ \pm a}$. By the construction of the lattices $\Lambda_{j, \lambda}^{i}$, the schematic closure of $T$ in $\prod_{i} \mathrm{GL}\left(\Lambda_{y}^{i}\right)$ is smooth. It follows by [11, Thm. 2.2.3] that the schematic closure $\mathcal{G}_{x}^{\prime}$ of $G$ in $\prod_{i} \mathrm{GL}\left(\Lambda_{y}^{i}\right)$ contains the smooth big open cell

$$
\prod_{a} \mathcal{U}_{-a} \times \mathcal{T} \times \prod_{a} \mathcal{U}_{a}
$$

Hence, by [11, Cor. 2.2.5], the schematic closure $\mathcal{G}_{x}^{\prime}$ is smooth. 
Remark 1.3.7. The above is similar to corresponding arguments in [26, §10], [27, §9]. Our assumption that $\rho: G \rightarrow \mathrm{GL}(V)$ is minuscule is used in an essential way in this proof. For example, the assumption that the weight spaces have dimension one is used to reduce to the case of $\mathrm{SL}_{2}$ : In general, for $G$ split semi-simple and $\rho$ irreducible, consider $\rho_{a}: \mathrm{SL}_{2} \rightarrow \mathrm{GL}(V)$ as before which we write as a direct sum of irreducible representations $V=\oplus_{t} V_{t}$. If $\operatorname{dim}\left(V_{\lambda}\right) \neq 1$, we might have two distinct summands $V_{t_{1}}, V_{t_{2}}$, with $V_{\lambda} \cap V_{t_{1}} \neq(0), V_{\lambda} \cap V_{t_{2}} \neq(0)$. Then we cannot guarantee that $\Lambda_{y}^{i}$ is equal to the direct sum $\oplus_{t}\left(\Lambda_{y}^{i} \cap V_{t}\right)$.

2) Assume now that $G$ is still split over $K$ but is not necessarily semi-simple. The argument above extends to this more general case by observing the following. The $\mathcal{O}^{\text {ur }}$-points $\mathcal{T}\left(\mathcal{O}^{\text {ur }}\right)$ of the Zariski closure $\mathcal{T}$ of $T$ in $\mathcal{G} \mathcal{L}_{y}$ give the maximal compact subgroup of $T\left(K^{\mathrm{ur}}\right)$. (Indeed, $\operatorname{Aut}\left(\Lambda_{y}^{\bullet}\right) \cap G\left(K^{\mathrm{ur}}\right)$ is equal to $G\left(K^{\mathrm{ur}}\right)_{x}=\mathcal{G}_{x}\left(\mathcal{O}^{\mathrm{ur}}\right)$ and since $x$ is in the apartment of $T$, the subgroup $G\left(K^{\mathrm{ur}}\right)_{x}$ contains the maximal compact subgroup of $T\left(K^{\mathrm{ur}}\right)$.) Then the Zariski closure $\mathcal{T}$ is smooth by 61, Lemma 4.1]. The rest is as before, since the unipotent subgroups $U_{a}$ and their Zariski closures $\mathcal{U}_{a}$ are the same for both $G$ and $G^{\text {der }}$.

3) We now consider the general case in which $G$ splits over the tamely ramified Galois extension $\tilde{K}$ of $K$ with group $\Gamma=\operatorname{Gal}(\tilde{K} / K)$. By [60, we have

$$
\mathcal{B}(G, K)=\mathcal{B}(G, \tilde{K})^{\Gamma}
$$

where on the right hand side, we have the fixed points of the natural action by $\Gamma$. For a bounded subset $\Omega \subset \mathcal{B}(G, K)$, the Galois group $\Gamma$ acts on $G(\tilde{K})_{\Omega}$. Since we are assuming $\tilde{K}=\tilde{K}^{\text {ur }}$, by [11, (1.7.6)], this action comes from an action of the Galois group $\Gamma$ on the smooth group scheme $\operatorname{Res}_{\tilde{\mathcal{O}} / \mathcal{O}}\left(\mathcal{G}_{\Omega, \tilde{K}}\right)$. (Here, we use the subscript $\tilde{K}$ to indicate that $\mathcal{G}_{\Omega, \tilde{K}}$ is the Bruhat-Tits group scheme over $\tilde{\mathcal{O}}$ which is associated to $\Omega$ considered as a subset of $\mathcal{B}(G, \tilde{K})$.)

Proposition 1.3.9. As above, suppose that $\tilde{K} / K$ is tamely ramified and Galois with Galois group $\Gamma$. Then we have

$$
\left(\operatorname{Res}_{\tilde{\mathcal{O}} / \mathcal{O}}\left(\mathcal{G}_{\Omega, \tilde{K}}\right)\right)^{\Gamma} \simeq \mathcal{G}_{\Omega, K},
$$

and in particular, a closed group scheme immersion

$$
\mathcal{G}_{\Omega, K} \hookrightarrow \operatorname{Res}_{\tilde{\mathcal{O}} / \mathcal{O}}\left(\mathcal{G}_{\Omega, \tilde{K}}\right) .
$$

Proof. Since $\left(G(\tilde{K})_{\Omega}\right)^{\Gamma}=\left(G(\tilde{K})^{\Gamma}\right)_{\Omega}=G(K)_{\Omega}$ this follows from [11, (1.7.6)] using that, by [22], the group scheme on the left hand side is smooth over $\mathcal{O}$.

By our construction of $\iota$ we have a commutative diagram where the horizontal arrows are equivariant toral embeddings

$$
\begin{aligned}
& \mathcal{B}(G, \tilde{K}) \rightarrow \prod_{j} \mathcal{B}\left(\operatorname{Res}_{K_{j 1} / K} \mathrm{GL}\left(V_{j}\right)_{D_{j}} \otimes_{K} \tilde{K}, \tilde{K}\right) \\
& \stackrel{\uparrow}{\wedge}(G, K) \rightarrow \prod_{j} \mathcal{B}\left(\operatorname{Res}_{K_{j 1} / K} \mathrm{GL}\left(V_{j}\right)_{D_{j}}, K\right) \quad \rightarrow \quad \mathcal{B}(\mathrm{GL}(V), K),
\end{aligned}
$$

and the vertical arrows are the natural embeddings. Here $K_{j 1}$ is the field obtained from $V_{j}$ and the representation $\rho_{j}: G \rightarrow \operatorname{GL}\left(V_{j}\right)$ over $K$. By our construction, the top horizontal arrow is the $G(\tilde{K})$-map of buildings $\left(\iota_{j, \sigma}\right)_{j, \sigma}$ that corresponds to

$$
\rho_{\tilde{K}}^{\prime}: G_{\tilde{K}} \rightarrow \prod_{j} \prod_{\sigma} \mathrm{GL}\left(V_{j} \otimes_{K_{j 1}} \tilde{K}\right)_{D_{j} \otimes_{K_{j 1}} \tilde{K}} \cong \prod_{j} \prod_{\sigma} \mathrm{GL}\left(V\left(\lambda_{j 1}\right) \otimes_{\mathbb{Q}_{p}} \tilde{K}\right) .
$$


(Here $\sigma$ runs over all $K$-embeddings $K_{j 1} \rightarrow \tilde{K}$ and $\rho_{\tilde{K}}^{\prime}$ can be identified with the product over $j$ of the base changes of (1.2.18) from $K$ to $\tilde{K}$.) Each factor corresponds to a minuscule irreducible $\tilde{K}$-representation of the split group $G_{\tilde{K}}$ and we can see that $\rho_{\tilde{K}}^{\prime}$ is faithful. The result in the split case implies that $\rho_{\tilde{K}}^{\prime}$ induces a closed immersion

$$
\rho_{\tilde{K}}^{\prime}: \mathcal{G}_{x, \tilde{K}} \hookrightarrow \prod_{j} \prod_{\sigma} \mathcal{G} \mathcal{L}\left(V\left(\lambda_{j}\right) \otimes_{\mathbb{Q}_{p}} \tilde{K}\right)_{\iota_{j, \sigma}(x)}
$$

of smooth group schemes over $\mathcal{O}_{\tilde{K}}$. Now, as in (1.3.10), we also have a closed immersion

$$
\operatorname{Res}_{\mathcal{O}_{j 1} / \mathcal{O}}\left(\left(\mathcal{G L}\left(V_{j}\right)_{D_{j}}\right)_{\iota_{j}(x)}\right) \hookrightarrow \prod_{\sigma} \operatorname{Res}_{\tilde{\mathcal{O}} / \mathcal{O}}\left(\mathcal{G} \mathcal{L}\left(V\left(\lambda_{j}\right) \otimes_{\mathbb{Q}_{p}} \tilde{K}\right)_{\iota_{j, \sigma}(x)}\right) .
$$

Since, again by (1.3.10), $\mathcal{G}_{x}=\mathcal{G}_{x, K} \rightarrow \operatorname{Res}_{\tilde{\mathcal{O}} / \mathcal{O}} \mathcal{G}_{x, \tilde{K}}$ is a closed immersion, we deduce that

$$
\mathcal{G}_{x, K} \rightarrow \prod_{j} \operatorname{Res}_{\mathcal{O}_{j 1} / \mathcal{O}}\left(\left(\mathcal{G L}\left(V_{j}\right)_{D_{j}}\right)_{\iota_{j}(x)}\right)
$$

is a closed immersion. The result now follows using [12, 3.5, 3.9]; this implies that the natural

$$
\operatorname{Res}_{\mathcal{O}_{j 1} / \mathcal{O}}\left(\left(\mathcal{G L}\left(V_{j}\right)_{D_{j}}\right)_{\iota_{j}(x)}\right) \rightarrow \mathcal{G L}\left(V_{j}\right)_{\iota_{j}(x)}
$$

corresponding to restriction of scalars

$$
\operatorname{Res}_{K_{j 1} / K}\left(\mathrm{GL}\left(V_{j}\right)_{D_{j}}\right) \rightarrow \operatorname{Res}_{K_{j 1} / K}\left(\mathrm{GL}\left(V_{j}\right)_{K_{j 1}}\right) \rightarrow \mathrm{GL}\left(V_{j}\right)
$$

(cf. (1.2.19) ) is a closed immersion.

1.3.13. We can see that the statement of Proposition 1.3 .3 continues to hold, with the same proof, in the equicharacteristic case $K=k((\pi))$ provided we consider $\rho: G \rightarrow \mathrm{GL}(V)$ and a corresponding embedding $\iota: \mathcal{B}\left(G, K^{\text {ur }}\right) \rightarrow \mathcal{B}\left(\mathrm{GL}(V), K^{\text {ur }}\right)$ which are given as in 1.2.27.

\subsection{Extending torsors.}

1.4.1. We continue to use the notation introduced above. Let $\mathcal{O}_{\mathcal{E}}$ be the $p$-adic completion of $W \llbracket u \rrbracket_{(p)}$; this is a henselian discrete valuation ring with residue field $k((u))$ and fraction field $\mathcal{E}=\mathcal{O}_{\mathcal{E}}[1 / p]=K_{0}\{\{u\}\}$. For simplicity, we set $D=$ $\operatorname{Spec}(W \llbracket u \rrbracket), D^{\times}=D-\{(u, p)\}$ and also $D[1 / p]=D^{\times}[1 / p]=\operatorname{Spec}(W \llbracket u \rrbracket[1 / p])$.

1.4.2. Suppose $G$ is a connected reductive group over $K_{0}$, and let $\mathcal{G}$ be a parahoric Bruhat-Tits (smooth) group scheme over $W$ for $G$; i.e. $\mathcal{G}=\mathcal{G}_{x}^{\circ}$ for a point $x$ in the Bruhat-Tits building $\mathcal{B}\left(G, K_{0}\right)$ and $G=\mathcal{G}[1 / p]=\mathcal{G} \otimes_{W} K_{0}$.

As above, we assume that $G$ splits over a tamely ramified extension of $K_{0}$. We also assume that $G$ has no factors of type $E_{8}$. (For our purposes, this is an acceptable assumption since it is satisfied for the reductive groups corresponding to Shimura varieties.) The main result of this section is the proof of the following:

Proposition 1.4.3. Under the above assumptions, each $\mathcal{G}$-torsor over $D^{\times}$is trivial, i.e. we have $\mathrm{H}^{1}\left(D^{\times}, \mathcal{G}\right)=(1)$.

Remark 1.4.4. When $x$ is hyperspecial (so in particular $G$ is quasi-split and split over an unramified extension of $K_{0}$, this follows from [16] as shown in [43]. See also Remark 1.4.15 below.

Before giving the proof of the Proposition, we need the following two Lemmas. In the arguments below, all the cohomology groups/sets are for the fppf topology. However, since all the coefficients here are given by smooth group schemes we could also use the étale topology with no change. 
Lemma 1.4.5. Let $Q$ be an induced torus over $K_{0}$, and $\mathcal{Q}^{\circ}$ its connected Néron model over $\mathcal{O}_{K_{0}}$. Then we have

$$
\mathrm{H}^{1}(D[1 / p], Q)=\{1\}
$$

and

$$
\operatorname{Im}\left(\mathrm{H}^{2}\left(D^{\times}, \mathcal{Q}^{\circ}\right) \rightarrow \mathrm{H}^{2}(D[1 / p], Q)\right)=\{1\} .
$$

Proof. By assumption $Q$ is a product of tori of the form $\operatorname{Res}_{K / K_{0}} \mathbb{G}_{\mathrm{m}}$, for $K / K_{0}$ a finite extension, and we may assume $Q=\operatorname{Res}_{K / K_{0}} \mathbb{G}_{\mathrm{m}}$. For the first claim we have

$$
\mathrm{H}^{1}(D[1 / p], Q)=\mathrm{H}^{1}\left(\operatorname{Spec} \mathcal{O}_{K} \llbracket u \rrbracket[1 / p], \mathbb{G}_{\mathrm{m}}\right)=\{1\}
$$

as $\mathcal{O}_{K} \llbracket u \rrbracket[1 / p]$ is a UFD.

For the second claim, note that we have a tautological character $\left.Q\right|_{K} \rightarrow \mathbb{G}_{\mathrm{m}}$, which extends to a map of smooth groups over $\mathcal{O}_{K},\left.\mathcal{Q}^{\circ}\right|_{\mathcal{O}_{K}} \rightarrow \mathbb{G}_{\mathrm{m}}$ since $\mathbb{G}_{\mathrm{m}}$ is the connected Néron model of its generic fibre. Finally, we obtain a map $\mathcal{Q}^{\circ} \rightarrow \operatorname{Res}_{\mathcal{O}_{K} / \mathcal{O}_{K_{0}}} \mathbb{G}_{\mathrm{m}}$, and it suffices to show that $\mathrm{H}^{2}\left(D^{\times}, \operatorname{Res}_{\mathcal{O}_{K} / \mathcal{O}_{K_{0}}} \mathbb{G}_{\mathrm{m}}\right)=\{1\}$, or equivalently $\mathrm{H}^{2}\left(D_{\mathcal{O}_{K}}^{\times}, \mathbb{G}_{\mathrm{m}}\right)=\{1\}$.

By purity of the Brauer group (e.g [33, Part II, Prop. 2.3] or [33, part III, Thm 6.1 (b)], and the fact that $\mathcal{O}_{K} \llbracket u \rrbracket$ is strictly henselian, we have

$$
\mathrm{H}^{2}\left(D_{\mathcal{O}_{K}}^{\times}, \mathbb{G}_{\mathrm{m}}\right)=\mathrm{H}^{2}\left(D_{\mathcal{O}_{K}}, \mathbb{G}_{\mathrm{m}}\right)=\mathrm{H}^{2}\left(\operatorname{Gal}(\bar{k} / k), \mathbb{G}_{\mathrm{m}}\right) .
$$

Our assumptions on $k$ imply the final group is trivial.

Lemma 1.4.6. Suppose that $G$ is quasi-split, semi-simple and simply connected with no factors of type $E_{8}$. Then $\mathrm{H}^{1}(D[1 / p], G)=\{1\}$.

Proof. Note that $D[1 / p]=\operatorname{Spec}(W \llbracket u \rrbracket[1 / p])$ is regular Noetherian of dimension 1. Set $\mathcal{K}=\operatorname{Frac}(W \llbracket u \rrbracket)$. This is a field of cohomological dimension 2: Indeed, if $\ell \neq p$, the $\ell$-cohomological dimension (see 67]) $\mathrm{cd}_{\ell}(\mathcal{K})$ of $\mathcal{K}$ is 2 by results of Gabber. (This verifies a conjecture of M. Artin, see [SGA4 X] or [40, Exp. XVIII].) On the other hand, $\operatorname{cd}_{p}(\mathcal{K})=2$ was shown by Kato, see [47, or 24 for a more general result. We now use results on Serre's conjecture II: By [28, if $H$ is a semi-simple, simply connected quasi-split reductive group with no $E_{8}$ factors, then $\mathrm{H}^{1}(\mathcal{K}, H)=(1)$. (This uses earlier more general results for groups of classical type, by Bayer-Fluckinger-Parimala, see [2, 3]. See also 30 for a survey.) Therefore, $\mathrm{H}^{1}(\mathcal{K}, G)=\{1\}$.

Now let $B \subset G$ be a Borel and $T \subset G$ a maximal torus. Let $J \rightarrow D[1 / p]$ be a $G$-torsor. Since $\mathrm{H}^{1}(\mathcal{K}, G)=(1), J$ has a section defined on a non-empty open subscheme $U$ of $D[1 / p]$. This gives a section of the associated $G / B$-bundle $J \times{ }^{G}$ $G / B \rightarrow U$. Since $D[1 / p]$ is affine, regular of Krull dimension 1 and $J \times{ }^{G} G / B \rightarrow U$ is proper, this section extends to a section defined over $D[1 / p]$. This defines a reduction of the structure group of $J$ from $G$ to $B$, i.e. a $B$-torsor $J^{\prime} \rightarrow D[1 / p]$ so that $J \simeq J^{\prime} \times{ }^{B} G$. Now notice that all $B$-torsors over $D[1 / p]$ are trivial. Indeed, $B$ is a successive extension of the maximal torus $T$ and unipotent groups of the form $\operatorname{Res}_{K^{\prime} / K_{0}} \mathbb{G}_{a}$. By an argument as in Lemma 1.4.5, all torsors for these unipotent groups are trivial Similarly, since the torus $T$ is induced, $\mathrm{H}^{1}(D[1 / p], T)=\{1\}$ by Lemma 1.4.5. It follows that the $G$-torsor $J$ is trivial; hence $\mathrm{H}^{1}(D[1 / p], G)=\{1\}$.

Proof of Proposition 1.4.3. Suppose that $\mathcal{J} \rightarrow D^{\times}$is a $\mathcal{G}$-torsor. We begin by considering the case when $k$ is algebraically closed. Then, by Steinberg's theorem 
$G$ is quasi-split, i.e. it contains a Borel subgroup $B$ defined over $K_{0}$. The variety of Borel subgroups $G / B$ is projective over $K_{0}$.

Step 1. The base change $\mathcal{J}_{\mathcal{O}_{\mathcal{E}}} \rightarrow \operatorname{Spec}\left(\mathcal{O}_{\mathcal{E}}\right)$ is a trivial $\mathcal{G} \otimes_{\mathbb{Z}_{p}} \mathcal{O}_{\mathcal{E}}$-torsor.

Indeed, the fibre $\mathcal{J}_{k((u))} \rightarrow \operatorname{Spec}(k((u)))$ is a trivial $\mathcal{G} \otimes_{W} k((u))$-torsor: This last group is an extension of a connected reductive group by a unipotent group both defined over $k$. (Recall here that the special fibre of $\mathcal{G}=\mathcal{G}_{x}^{\circ}$ is connected.) Since the cohomological dimension of $k((u))$ is 1 ([67, II 3.3]), the result then follows by Steinberg's theorem ([67, III. 2.3, Remark 1)]) and the fact that $\mathrm{H}^{1}\left(k((u)), \mathbb{G}_{a}\right)=$ $\{0\}$. Since $\mathcal{O}_{\mathcal{E}}$ is henselian with residue field $k((u))$ and $\mathcal{J}_{\mathcal{O}_{\mathcal{E}}} \rightarrow \operatorname{Spec}\left(\mathcal{O}_{\mathcal{E}}\right)$ is smooth, a section of $\mathcal{J}_{\mathcal{O}_{\mathcal{E}}}$ over $k((u))$ lifts to a section over $\mathcal{O}_{\mathcal{E}}$.

Step 2. The base change $\mathcal{J}[1 / p] \rightarrow D[1 / p]$ is a trivial $G$-torsor.

By [15, there is a flasque resolution

$$
1 \rightarrow Z \rightarrow \tilde{G} \rightarrow G \rightarrow 1
$$

with $Z$ a flasque (central) torus, $\tilde{G}^{\text {der }}$ semi-simple simply connected and at the same time

$$
1 \rightarrow \tilde{G}^{\mathrm{der}} \rightarrow \tilde{G} \rightarrow Q \rightarrow 1
$$

with $Q$ an induced torus (i.e. $Q \simeq \prod_{i} \operatorname{Res}_{K_{i} / K_{0}} \mathbb{G}_{\mathrm{m}}$ where $K_{i} / K_{0}$ are finite tamely ramified extensions). Recall that a torus $Z$ over $K_{0}$ is called flasque if for every open subgroup $I^{\prime} \subset I=\operatorname{Gal}\left(\bar{K}_{0} / K_{0}\right)$ we have $\mathrm{H}^{1}\left(I^{\prime}, \mathbb{X} \bullet(Z)\right)=0$. Since $Z$ is central, it is contained in the centralizer $\tilde{T}=Z(\tilde{S})$ of any maximal $K_{0}$-split torus $\tilde{S}$ of $\tilde{G}$. Actually, in this case we see (loc. cit.) that these centralizer maximal tori of both $\tilde{G}^{\text {der }}$ and $\tilde{G}$ are induced. (This will be used later). Since we are assuming that $G$ splits after a tamely ramified (and hence cyclic) extension of $K_{0}$ the flasque torus $Z$ is also a direct summand of an induced torus (see [14, Prop. 1]; this uses a result of Endo-Miyata on permutation Galois modules) so we have $Z \times_{K_{0}} Z^{\prime} \simeq Q^{\prime}$ for some torus $Z^{\prime}$, and with $Q^{\prime}$ an induced torus.

By Lemmas 1.4.5 and 1.4.6, we have $\mathrm{H}^{1}(D[1 / p], \tilde{G})=\{1\}$. Hence it suffices to show that the image of $\mathcal{J}$ in $\mathrm{H}^{2}(D[1 / p], Z)$ is trivial. By Proposition 1.1.4 there is an exact sequence of smooth group schemes over $W$

$$
1 \rightarrow \mathcal{Z}^{\circ} \rightarrow \tilde{\mathcal{G}} \rightarrow \mathcal{G} \rightarrow 1
$$

where $\mathcal{Z}$ is the finite type Neron model $([11, \S 4.4])$ of the torus $Z$ and $\tilde{\mathcal{G}}=\tilde{\mathcal{G}}_{x}^{\circ}$ is the parahoric group scheme for the group $\tilde{G}$ that corresponds to $\bar{x} \in \mathcal{B}\left(G^{\text {ad }}, K_{0}\right)=$ $\mathcal{B}\left(\tilde{G}^{\text {ad }}, K_{0}\right)$. Hence the image of $\mathcal{J}$ in $\mathrm{H}^{2}(D[1 / p], Z)$ is its image under the composite map

$$
\mathrm{H}^{1}\left(D^{\times}, \mathcal{G}\right) \rightarrow \mathrm{H}^{2}\left(D^{\times}, \mathcal{Z}^{\circ}\right) \rightarrow \mathrm{H}^{2}(D[1 / p], Z) .
$$

This is trivial by Lemma 1.4.5, since $Z$ is a direct summand of an induced torus.

Step 3. We have $G(W \llbracket u \rrbracket[1 / p]) \backslash G(\mathcal{E}) / \mathcal{G}\left(\mathcal{O}_{\mathcal{E}}\right)=\{1\}$.

Assuming this, let us show that the $\mathcal{G}$-torsor $\mathcal{J}$ is trivial. Indeed, from Steps 1 and 2 we have sections $a_{p}$ and $a[1 / p]$ of the torsor $\mathcal{J} \rightarrow D^{\times}$over $\mathcal{O}_{\mathcal{E}}$ and $D[1 / p]$ respectively. Consider $g \cdot a_{p}=a[1 / p], g \in G(\mathcal{E})$, with both sections restricted on Spec $(\mathcal{E})$. The triviality of the double cosets above implies that we can modify these sections to achieve $g=1$. Now observe that $D[1 / p] \sqcup \operatorname{Spec}\left(\mathcal{O}_{\mathcal{E}}\right) \rightarrow D^{\times}$is a cover in the fpqc topology; by Grothendieck's theorem on full faithfulness of fpqc descent data (e.g. [6, Chapter 6, Thm. 6 (a)]) we can conclude that the torsor $\mathcal{J}$ is trivial over $D^{\times}$, cf. [29, Appendix]. 
We now turn to showing the triviality of the double cosets above. Pick an alcove in the Bruhat-Tits building of $G$ over $K_{0}$ whose closure contains $x$. The connected stabilizer of any point in that alcove is an Iwahori subgroup $\mathcal{I}$ for which $\mathcal{I}\left(\mathcal{O}_{\mathcal{E}}\right) \subset \mathcal{G}\left(\mathcal{O}_{\mathcal{E}}\right)$. This shows that it is enough to assume that $\mathcal{G}=\mathcal{I}$, i.e. that $\mathcal{G}$ is an Iwahori group scheme.

Now denote by $W_{a}$ the affine Weyl group of $G\left(K_{0}\right)$, which is generated by the simple reflections $s_{i}, i=1, \ldots, m$, along the walls of the alcove. The reflections $s_{i}$ are all represented by elements of $G\left(K_{0}\right)$. There are corresponding parahorics $\mathcal{G}_{i}$ such that $\mathcal{I}(W)=\mathcal{G}(W) \subset \mathcal{G}_{i}(W)$ with $\mathcal{G}_{i}(W)=\mathcal{G}(W) \sqcup \mathcal{G}(W) s_{i} \mathcal{G}(W)$. Then also $\mathcal{G}_{i}\left(\mathcal{O}_{\mathcal{E}}\right)=\mathcal{G}\left(\mathcal{O}_{\mathcal{E}}\right) \sqcup \mathcal{G}\left(\mathcal{O}_{\mathcal{E}}\right) s_{i} \mathcal{G}\left(\mathcal{O}_{\mathcal{E}}\right)$. The cosets $\mathcal{G}_{i}\left(\mathcal{O}_{\mathcal{E}}\right) / \mathcal{G}\left(\mathcal{O}_{\mathcal{E}}\right)$ are parametrized by the $k((u))$-valued points of the projective line $\mathbb{P}^{1}$ : To be more precise, let $G_{i}$ be the maximal reductive quotient of the special fibre $\overline{\mathcal{G}}_{i}=\mathcal{G}_{i} \otimes_{W} k$. The derived group $G_{i}^{\text {red }}$ of $G_{i}$ is $\mathrm{SL}_{2}$ or $\mathrm{PSL}_{2}$. Since $\mathcal{G}_{i}$ is smooth and $\mathcal{O}_{\mathcal{E}}$ is $p$-adically complete, reduction modulo $p$ gives surjective homomorphisms

$$
\mathcal{G}_{i}\left(\mathcal{O}_{\mathcal{E}}\right) \rightarrow \overline{\mathcal{G}}_{i}(k((u))) \text {. }
$$

Composing this with the surjection $\overline{\mathcal{G}}_{i}(k((u))) \rightarrow G_{i}(k((u)))$ gives surjective homomorphisms

$$
\mathcal{G}_{i}\left(\mathcal{O}_{\mathcal{E}}\right) \stackrel{q_{i}}{\longrightarrow} G_{i}(k((u))) \rightarrow 1 .
$$

Now the group $\mathcal{G}\left(\mathcal{O}_{\mathcal{E}}\right)$ is the inverse image by $q_{i}$ of a Borel subgroup $B_{i}(k((u))) \subset$ $G_{i}(k((u)))$. This gives

$$
\mathcal{G}_{i}\left(\mathcal{O}_{\mathcal{E}}\right) / \mathcal{G}\left(\mathcal{O}_{\mathcal{E}}\right) \stackrel{\sim}{\rightarrow} G_{i}(k((u))) / B_{i}(k((u))) \simeq \mathbb{P}^{1}(k((u))) .
$$

Similarly, since $\mathcal{G}_{i}$ is smooth over $W$, by Hensel's lemma, reduction modulo $p$ gives a surjective homomorphism $\mathcal{G}_{i}(W \llbracket u \rrbracket) \rightarrow \overline{\mathcal{G}}_{i}(k \llbracket u \rrbracket)$. As before, we obtain similar surjective homomorphisms

$$
\mathcal{G}_{i}(W \llbracket u \rrbracket) \stackrel{q_{i}}{\longrightarrow} G_{i}(k \llbracket u \rrbracket) \rightarrow 1
$$

which give

$$
\mathcal{G}_{i}(W \llbracket u \rrbracket) / \mathcal{G}(W \llbracket u \rrbracket) \stackrel{\sim}{\rightarrow} \mathbb{P}^{1}(k \llbracket u \rrbracket) .
$$

These isomorphisms (1.4.10) and (1.4.11) are compatible via $W \llbracket u \rrbracket \rightarrow \mathcal{O}_{\mathcal{E}}$, which modulo $p$ induces $k \llbracket u \rrbracket \rightarrow k((u))$. Since $\mathbb{P}^{1}$ is proper, $\mathbb{P}^{1}(k \llbracket u \rrbracket)=\mathbb{P}^{1}(k((u)))$. Hence if $x_{i} \in \mathcal{G}_{i}\left(\mathcal{O}_{\mathcal{E}}\right) / \mathcal{G}\left(\mathcal{O}_{\mathcal{E}}\right)$, we can find $g_{i} \in \mathcal{G}_{i}(W \llbracket u \rrbracket) \subset G(W \llbracket u \rrbracket[1 / p])$ so that $x_{i}=$ $g_{i} \cdot \mathcal{G}\left(\mathcal{O}_{\mathcal{E}}\right)$. Hence

$$
\mathcal{G}_{i}\left(\mathcal{O}_{\mathcal{E}}\right)=\mathcal{G}_{i}(W \llbracket u \rrbracket) \cdot \mathcal{G}\left(\mathcal{O}_{\mathcal{E}}\right) .
$$

Now, for each $n \geq 1$, and $i_{1}, \ldots, i_{n}$ integers in $[1, m]$, consider the map

$$
\mathcal{G}_{i_{1}}\left(\mathcal{O}_{\mathcal{E}}\right) \times \cdots \times \mathcal{G}_{i_{n}}\left(\mathcal{O}_{\mathcal{E}}\right) \rightarrow G(\mathcal{E}) / \mathcal{G}\left(\mathcal{O}_{\mathcal{E}}\right), \quad\left(y_{1}, \ldots, y_{n}\right) \mapsto y_{1} \cdots y_{n} \cdot \mathcal{G}\left(\mathcal{O}_{\mathcal{E}}\right)
$$

which factors via the quotient by the action of $\mathcal{G}\left(\mathcal{O}_{\mathcal{E}}\right)^{n}$ given by

$$
\left(y_{1}, \ldots, y_{n}\right) \cdot\left(p_{1}, \ldots, p_{n}\right)=\left(y_{1} p_{1}, p_{1}^{-1} y_{2} p_{2}, \ldots, p_{n-1}^{-1} y_{n} p_{n}\right) .
$$

Start with $\left(y_{1}, \ldots, y_{n}\right)$ as above. By (1.4.12), there is $p_{1} \in \mathcal{G}\left(\mathcal{O}_{\mathcal{E}}\right)$ so that $y_{1} p_{1}=$ $g_{1} \in \mathcal{G}_{i_{1}}(W \llbracket u \rrbracket)$. Consider $p_{1}^{-1} y_{2} \in \mathcal{G}\left(\mathcal{O}_{\mathcal{E}}\right) \mathcal{G}_{i_{2}}\left(\mathcal{O}_{\mathcal{E}}\right) \subset \mathcal{G}_{i_{2}}\left(\mathcal{O}_{\mathcal{E}}\right)$. Applying (1.4.12) again, we see that there is $p_{2} \in \mathcal{G}\left(\mathcal{O}_{\mathcal{E}}\right)$ so that $p_{1}^{-1} y_{2} p_{2}=g_{2} \in \mathcal{G}_{i_{2}}(W \llbracket u \rrbracket)$. Continuing, we find $\left(g_{1}, \ldots, g_{n}\right)$ and $\left(p_{1}, \ldots, p_{n}\right) \in \mathcal{G}\left(\mathcal{O}_{\mathcal{E}}\right)^{n}$ with $g_{k} \in \mathcal{G}_{i_{k}}(W \llbracket u \rrbracket) \subset$ $G(W \llbracket u \rrbracket[1 / p])$ and $\left(y_{1}, \ldots, y_{n}\right) \cdot\left(p_{1}, \ldots, p_{n}\right)=\left(g_{1}, \ldots, g_{n}\right)$. This gives

$$
y_{1} y_{2} \cdots y_{n} \cdot \mathcal{G}\left(\mathcal{O}_{\mathcal{E}}\right)=g_{1} g_{2} \cdots g_{n} \cdot \mathcal{G}\left(\mathcal{O}_{\mathcal{E}}\right) \text {. }
$$


Denote by $G(\mathcal{E})^{1}$ the subgroup of $G(\mathcal{E})$ generated by all the parahoric subgroups $\mathcal{G}_{i}\left(\mathcal{O}_{\mathcal{E}}\right)$. The above calculation implies that the image of $G(W \llbracket u \rrbracket[1 / p])$ in $G(\mathcal{E}) / \mathcal{G}\left(\mathcal{O}_{\mathcal{E}}\right)$ contains $G(\mathcal{E})^{1} / \mathcal{G}\left(\mathcal{O}_{\mathcal{E}}\right)$.

The group $G(\mathcal{E})^{1}$ coincides with the subgroup generated by all parahoric subgroups of $G(\mathcal{E})$ considered in [11, 5.2.11]. As above, choose a maximal split torus $S \subset G$ whose apartment contains $x$, and let $\mathcal{T} \subset \mathcal{G}_{x}$ be the closure of its centralizer $T=Z_{G}(S)$. By loc. cit. 5.2.4, $G(\mathcal{E})^{1}$ is also the subgroup generated by $\mathcal{T}^{\circ}\left(\mathcal{O}_{\mathcal{E}}\right)$ and the $\mathcal{E}$-valued points of the root subgroups of $G$. (Notice that we have $\mathcal{T}^{\circ}\left(\mathcal{O}_{\mathcal{E}}\right) \subset \mathcal{G}\left(\mathcal{O}_{\mathcal{E}}\right) \subset G(\mathcal{E})^{1}$.) We now have

$$
G(\mathcal{E})=T(\mathcal{E}) \cdot G(\mathcal{E})^{1}
$$

Now consider the quotient $T(\mathcal{E}) / \mathcal{T}^{\circ}\left(\mathcal{O}_{\mathcal{E}}\right)$. The natural homomorphism $T\left(K_{0}\right) \rightarrow$ $T(\mathcal{E})$ gives a surjection $T\left(K_{0}\right) / \mathcal{T}^{\circ}(W) \rightarrow T(\mathcal{E}) / \mathcal{T}^{\circ}\left(\mathcal{O}_{\mathcal{E}}\right)$. When the torus $T$ is induced this follows from [11, 4.4.14]. In our more general case, we have, as in Step $2, T=\tilde{T} / Z$ with $\tilde{T}$ induced and $Z$ flasque. As above, since $Z$ is a direct summand of an induced torus, we have $\mathrm{H}^{1}(\mathcal{E}, Z)=(1)$. Hence, $\tilde{T}(\mathcal{E}) \rightarrow T(\mathcal{E})$ is surjective and the desired surjectivity above then follows from the corresponding property for $\tilde{T}$. Therefore, 1.4.13) gives

$$
G(\mathcal{E})=T\left(K_{0}\right) \cdot G(\mathcal{E})^{1} .
$$

Since $T\left(K_{0}\right) \subset G(W \llbracket u \rrbracket[1 / p])$, this completes the proof of the proposition in the case when $k$ is algebraically closed.

Step 4. The proposition holds for any $k$.

Write $D_{\mathcal{O}_{L}}=\operatorname{Spec} \mathcal{O}_{L} \llbracket u \rrbracket$ and $D_{\mathcal{O}_{L}}^{\times} \subset D_{\mathcal{O}_{L}}$ the complement of the closed point. Denote by $f: D_{\mathcal{O}_{L}} \rightarrow D$ the projection. Then $f^{*}(\mathcal{J})$ is equipped with a $\mathcal{G}$ equivariant descent datum for the morphism $f$. By what we have already seen $f^{*}(\mathcal{J})$ is a trivial $\mathcal{G}$-torsor over $D_{\mathcal{O}_{L}}^{\times}$, and we may consider this descent datum as a descent datum on $\mathcal{G} \times{ }_{D} \times D_{\mathcal{O}_{L}}^{\times}$. Since $\mathcal{G}$ is affine this extends to an effective, $\mathcal{G}$ equivariant descent datum on $\mathcal{G} \times{ }_{D} D_{\mathcal{O}_{L}}$, which produces a $\mathcal{G}$-torsor on $\tilde{\mathcal{J}}$ over $D$ extending $\mathcal{J}$.

Finally $\tilde{\mathcal{J}}$ has a section over the closed point of $D$ by Lang's lemma, and hence over $D$ by smoothness. It follows that $\tilde{\mathcal{J}}$ and hence $\mathcal{J}$ is a trivial $\mathcal{G}$-torsor.

Remark 1.4.15. Under the additional hypothesis that $G$ is split over $K_{0}$ and that the subgroup $\mathcal{G}(W)=\mathcal{G}_{x}(W)$ is contained in a hyperspecial subgroup $G_{W}(W)$ we can give a quicker proof of Proposition 1.4.3. (Notice then that by [38, $\mathcal{G}_{x}^{\circ}=\mathcal{G}_{x}$, since the Kottwitz invariant homomorphism vanishes on $\mathcal{G}_{x}(W) \subset G_{W}(W)$.) We sketch the argument below:

Recall that there is a representation $G_{W} \hookrightarrow \mathrm{GL}_{n / W}$ which is a closed immersion such that the quotient $\mathrm{GL}_{n / W} / G_{W}$ is an affine scheme ([16, [43]). Under our assumption, there is a parabolic subgroup $Q \subset \bar{G}_{W}=G_{W} \otimes_{W} k$ such that $\mathcal{G}(W) \subset$ $G_{W}(W)$ is the preimage of $Q(k) \subset G_{W}(k)$. In this case, $\mathcal{G}$ is given as the dilatation ([6], 72]) of $G_{W} \rightarrow \operatorname{Spec}(W)$ along the subgroup $Q \subset G_{W} \otimes_{W} k$ of its special fibre.

We can now write $Q$ as the scheme theoretic intersection of $G_{W} \otimes_{W} k$ and a parabolic subgroup $Q^{\prime}$ in $\mathrm{GL}_{n / k}$. The dilation of $\mathrm{GL}_{n / W} \rightarrow \operatorname{Spec}(W)$ along $Q^{\prime}$ is a parahoric subgroup $\mathcal{G} \mathcal{L}_{y}$ scheme for $\mathrm{GL}_{n}$ which is given as the stabilizer of a corresponding lattice chain. We have a closed group scheme immersion $\mathcal{G} \hookrightarrow \mathcal{G} \mathcal{L}_{y}$ such that the quotient $\mathcal{G} \mathcal{L}_{y} / \mathcal{G}$ is affine: Indeed, the quotient $\mathcal{G} \mathcal{L}_{y} / \mathcal{G}$ can be identified as the dilatation of the affine scheme $\mathrm{GL}_{n / W} / G_{W}$ along the closed subscheme $Q^{\prime} / Q$ 
of its closed fibre. Such dilatations of affine schemes are also affine. Now use, as in [16], [43, the fact that any morphism $D^{\times} \rightarrow X$ with $X$ affine extends to $D \rightarrow X$ to reduce the proof to the case that the group is $G=\mathrm{GL}_{n}$ and the parahoric subgroup $\mathcal{G}=\mathcal{G} \mathcal{L}_{y}$.

When $\mathcal{G}=\mathcal{G L}_{y}$, a $\mathcal{G}$-torsor over a scheme $T$ is given (cf. 64, Appendix to Chapter 3]) by a periodic chain $\left(\mathcal{F}_{i}, \varphi_{i}\right)_{i}$ of locally free $\operatorname{rank} n \mathcal{O}_{T}$-modules $\mathcal{F}_{i}$ with maps $\varphi_{i}: \mathcal{F}_{i} \rightarrow \mathcal{F}_{i+1}$ such that, for all $i$, the quotients $\mathcal{F}_{i+1} / \varphi_{i}\left(\mathcal{F}_{i}\right)$ are locally free $\mathcal{O}_{T} / p \mathcal{O}_{T}$-modules of fixed rank $r_{i}$ (which depends on our choice of $y$ ). (By "periodic" one means that there is $a \geq 1$ such that $\mathcal{F}_{i+a}=\mathcal{F}_{i}$ and the composition $\varphi_{i+a-1} \cdots \varphi_{i+1} \cdot \varphi_{i}$ is multiplication by $p$, for all $i$.) Since $D$ is regular Noetherian of dimension 2 and $D-D^{\times}$has codimension 2 , a periodic chain $\left(\mathcal{F}_{i}, \varphi_{i}\right)$ over $D^{\times}$uniquely extends to a periodic chain $\left(\tilde{\mathcal{F}}_{i}, \tilde{\varphi}_{i}\right)$ over $D$. If $\left(\mathcal{F}_{i}, \varphi_{i}\right)$ satisfies the above condition on the quotients, then so does the extended chain $\left(\tilde{\mathcal{F}}_{i}, \tilde{\varphi}_{i}\right)$ : Indeed, by the above, the $W \llbracket u \rrbracket$-modules $\tilde{\mathcal{F}}_{i} / \tilde{\varphi}_{i}\left(\tilde{\mathcal{F}}_{i}\right)$ have projective dimension 1 , and are annihilated by $p$. By the Auslander-Buchsbaum theorem $\tilde{\mathcal{F}}_{i} / \tilde{\varphi}_{i}\left(\tilde{\mathcal{F}}_{i}\right)$ has only trivial $u$-torsion; therefore, it is free over $W \llbracket u \rrbracket / p W \llbracket u \rrbracket=k \llbracket u \rrbracket$. This establishes that the $\mathcal{G}$-torsor over $D^{\times}$extends to a $\mathcal{G}$-torsor over $D$ which then has to be trivial as before.

\section{LOCAL MODELS}

\subsection{The local models.}

2.1.1. We now recall the definition of the local models from [59, §7]. We continue to use the notation of the previous section, but we assume that $K / \mathbb{Q}_{p}$ is a finite unramified extension of $\mathbb{Q}_{p}$. Suppose that $(G,\{\mu\}, \mathrm{K})$ is a triple, with

- $G$ a connected reductive group over $K$,

- $\{\mu\}$ a conjugacy class of a geometric cocharacter $\mu: \mathbb{G}_{\mathrm{m}} \overline{\mathbb{Q}}_{p} \rightarrow G_{\overline{\mathbb{Q}}_{p}}$, and

- $\mathrm{K} \subset G(K)$ a parahoric subgroup which is the connected stabilizer of the point $x \in \mathcal{B}(G, K)$.

We assume that $G$ splits over a tame extension of $K$ and that $\mu$ is minuscule. ${ }^{3}$

Suppose that $E \subset \overline{\mathbb{Q}}_{p}$ is the local reflex field, i.e. the extension of $K$ which is the field of definition of the conjugacy class $\{\mu\}$.

In [59, §3], there is a construction of a smooth affine group scheme $\underline{\mathcal{G}}$ over $\mathcal{O}[u]$ which specializes to the parahoric group scheme $\mathcal{G}:=\mathcal{G}_{x}^{\circ}$ over $\mathcal{O}$ after the base change $\mathcal{O}[u] \rightarrow \mathcal{O}$ given by $u \mapsto p$ (loc. cit. $\S 4$ ), and such that $\underline{G}=\left.\underline{\mathcal{G}}\right|_{\mathcal{O}\left[u, u^{-1}\right]}$ is reductive. There is a corresponding ind-projective ind-scheme (the global affine Grassmannian) $\operatorname{Gr}_{\mathcal{G}, \mathbb{A}^{1}} \rightarrow \mathbb{A}^{1}=\operatorname{Spec}(\mathcal{O}[u])$ (loc. cit. $\S 6$ ). The base change $\operatorname{Gr}_{\underline{\mathcal{G}, \mathbb{A}^{1}}} \times_{\mathbb{A}^{1}} \operatorname{Spec}(\bar{K})$ under $\mathcal{O}[u] \rightarrow K$ given by $u \mapsto p$ can be identified with the affine Grassmannian $\operatorname{Gr}_{G, K}$ of $G$ over $K$. (Recall that $\mathrm{Gr}_{G, K}$ represents the fpqc sheaf associated to the quotient $R \mapsto G(R((t)) / G(R \llbracket t \rrbracket)$; the identification is via $t=u-p$.)

The cocharacter $\mu$ defines a projective homogeneous space $G_{\overline{\mathbb{Q}}_{p}} / P_{\mu^{-1}}$ over $\overline{\mathbb{Q}}_{p}$. Here, $P_{\nu}$ denotes the parabolic subgroup that corresponds to the coweight $\nu$; by definition, the Lie algebra $\operatorname{Lie}\left(P_{\nu}\right)$ contains all the root subgroups $U_{a}$ for roots $a$ such that $a \cdot \nu: \mathbb{G}_{\mathrm{m}} \rightarrow \mathbb{G}_{\mathrm{m}}$ is a non-negative power of the identity character. Since the conjugacy class $\{\mu\}$ is defined over $E$ we can see that this homogeneous

\footnotetext{
${ }^{3}$ Recall that $\mu$ is minuscule if $\langle a, \mu\rangle \in\{-1,0,1\}$ for every root $a$ of $G_{\overline{\mathbb{Q}}_{p}}$.
} 
space has a canonical model $X_{\mu}$ defined over $E$ (notice however, that $X_{\mu}$ might not have any $E$-rational point). If $G$ is quasi-split, then $\{\mu\}$ has a representative $\mu: \mathbb{G}_{\mathrm{m} E} \rightarrow G_{E}$ which is actually defined over $E$ [45, 1.1.3] then we can write $X_{\mu}=G_{E} / P_{\mu^{-1}}$ which has an $E$-rational point.

Since $\mu$ is minuscule, the corresponding affine Schubert variety with $\overline{\mathbb{Q}}_{p}$-points $S_{\mu}\left(\overline{\mathbb{Q}}_{p}\right)=G\left(\overline{\mathbb{Q}}_{p} \llbracket t \rrbracket\right) \mu(t) G\left(\overline{\mathbb{Q}}_{p} \llbracket t \rrbracket\right) / G\left(\overline{\mathbb{Q}}_{p} \llbracket t \rrbracket\right)$ in the affine Grassmannian $\operatorname{Gr}_{G, K} \times_{K}$ $\overline{\mathbb{Q}}_{p}$ is closed, see [58, p. 146]. Our assumption that the conjugacy class $\{\mu\}$ is defined over $E$ implies that $S_{\mu}\left(\overline{\mathbb{Q}}_{p}\right)$ is $\operatorname{Gal}\left(\overline{\mathbb{Q}}_{p} / E\right)$-equivariant and so it corresponds to a closed subvariety $S_{\mu}$ of the ind-projective $\operatorname{Gr}_{G, K} \times_{K} E$. The natural left action of $G\left(\overline{\mathbb{Q}}_{p} \llbracket t \rrbracket\right)$ on $S_{\mu}\left(\overline{\mathbb{Q}}_{p}\right)$ is transitive and the stabilizer of $\mu(t)$ is $H_{\mu}:=$ $G\left(\overline{\mathbb{Q}}_{p} \llbracket t \rrbracket\right) \cap \mu(t) G\left(\overline{\mathbb{Q}}_{p} \llbracket t \rrbracket\right) \mu(t)^{-1}$. Let $T$ be a maximal torus of $G_{\overline{\mathbb{Q}}_{p}}$ which contains the image of $\mu$. Then $H_{\mu}$ contains $T\left(\overline{\mathbb{Q}}_{p} \llbracket t \rrbracket\right)$. Since $\mu$ is minuscule, we can see that $H_{\mu}$ contains the kernel of the canonical homomorphism $U_{a}\left(\overline{\mathbb{Q}}_{p} \llbracket t \rrbracket\right) \rightarrow U_{a}\left(\overline{\mathbb{Q}}_{p}\right)$, for all roots $a$ of $G_{\overline{\mathbb{Q}}_{p}}$. We conclude that $H_{\mu}$ contains the kernel of $G\left(\overline{\mathbb{Q}}_{p} \llbracket t \rrbracket\right) \rightarrow G\left(\overline{\mathbb{Q}}_{p}\right)$; by definition, the image of $H_{\mu}$ in $G\left(\overline{\mathbb{Q}}_{p}\right)$ is equal to $P_{\mu^{-1}}\left(\overline{\mathbb{Q}}_{p}\right)$. Hence, $S_{\mu}$ can be $G_{E^{-}}$equivariantly identified with $X_{\mu}$.

The local model $\mathrm{M}_{G,\{\mu\}, x}^{\text {loc }}:=\mathrm{M}^{\mathrm{loc}}(G,\{\mu\})_{x}$ is by definition the Zariski closure of $X_{\mu} \subset \operatorname{Gr}_{G, K} \times_{K} E$ in $\operatorname{Gr}_{\underline{G}, \mathbb{A}^{1}} \times_{\mathbb{A}^{1}} \operatorname{Spec}\left(\mathcal{O}_{E}\right)$ where the base change $\mathcal{O}[u] \rightarrow \mathcal{O}_{E}$ is given by $u \mapsto p$. By its construction, $\mathrm{M}_{G,\{\mu\}, x}^{\text {loc }}$ is a projective flat scheme over $\operatorname{Spec}\left(\mathcal{O}_{E}\right)$ which admits an action of the group scheme $\mathcal{G} \times_{\mathcal{O}} \mathcal{O}_{E}$. We recall:

Theorem 2.1.2. (59, Theorem 9.1]) Suppose in addition that $p$ does not divide the order of the (algebraic) fundamental group $\pi_{1}\left(G^{\mathrm{der}}\right)$ of the derived group of $G$. Then the scheme $\mathrm{M}_{G,\{\mu\}, x}^{\text {loc }}$ is normal. The geometric special fibre of $\mathrm{M}_{G,\{\mu\}, x}^{\mathrm{loc}}$ is reduced and admits a stratification with locally closed smooth strata; the closure of each stratum is normal and Cohen-Macaulay.

Corollary 2.1.3. Under the above assumptions, the base change $\mathrm{M}_{G,\{\mu\}, x}^{\mathrm{loc}} \otimes_{\mathcal{O}_{E}} \mathcal{O}_{L}$ is normal, for every finite extension $L / E$.

Proof. Using Theorem 2.1.2, we see that the special fibre of $\mathrm{M}_{G,\{\mu\}, x}^{\mathrm{loc}} \otimes_{\mathcal{O}_{E}} \mathcal{O}_{L}$ is reduced. The result then follows as in [59, Prop. 9.2].

2.1.4. For simplicity, we will often write $\mathrm{M}_{G}^{\mathrm{loc}}$ instead of $\mathrm{M}_{G,\{\mu\}, x}^{\mathrm{loc}}$, when $\{\mu\}$ and $x$ are understood. When the data $(G,\{\mu\}, \mathrm{K})$ are obtained, as in the next chapters, from global Shimura data $\left(G, X, \prod_{l} \mathrm{~K}_{l}\right)$ after the choice of a prime $v \mid p$ of the reflex field $E(G, X)$, we will often write $\mathrm{M}_{G, X}^{\text {loc }}$ instead of $\mathrm{M}_{G,\{\mu\}, x}^{\text {loc }}$. In particular, in this we take $\mu$ to be in the conjugacy class of $\mu_{h}$ for $h \in X$.

We now recall some points of the construction of the group schemes $\underline{G}$ and $\underline{\mathcal{G}}$. We will only need these details when the reductive group $G$ is quasi-split over $K$; then this construction is somewhat more straightforward and proceeds as follows. (Notice that Steinberg's theorem implies that we can make sure that this assumption is always satisfied after enlarging the unramified extension $K / \mathbb{Q}_{p}$.)

Choose a maximal $K$-split torus $S$ of $G$. Since $G$ is quasi-split, the centralizer $T=Z_{G}(S)$ is a maximal torus of $G$. Also choose a Borel subgroup $B$ of $G$ defined over $K$ that contains $S$ and consider the corresponding based root datum $\mathcal{R}^{+}:=$ ( $\left.\mathbb{X}_{\bullet}(T), \Delta, \mathbb{X}^{\bullet}(T), \Delta^{+}\right)$where $\Delta \subset \Phi$ is the set of simple roots that corresponds to $B$ in the root system $\Phi=\Phi(G, T)$. Denote again by $H$ the split Chevalley form 
of $G$ over $\mathbb{Z}_{p}$, and choose a pinning $\left(T_{H}, B_{H}, \underline{e}\right)$ of $H$ over $\mathbb{Z}_{p}$. The corresponding based root datum of $H$ agrees with $\mathcal{R}^{+}$. Set $\Xi:=\Xi_{H}=\operatorname{Aut}\left(\mathcal{R}^{+}\right)$.

The quasi-split group $G$ over $K$ is described by a $\Xi_{H}$-torsor over $K$; this splits over a tame finite extension $K \subset \tilde{K} \subset \overline{\mathbb{Q}}_{p}$ and can thus by described via a group homomorphism $\xi: \operatorname{Gal}\left(\overline{\mathbb{Q}}_{p} / K\right) \rightarrow \Xi$ that factors through $\Gamma_{K}=\operatorname{Gal}(\tilde{K} / K)$. As explained in [59, 3.2, 3.3], $\xi$ corresponds to $\underline{\xi}: \pi_{1}\left(\operatorname{Spec}\left(\mathcal{O}\left[u^{ \pm 1}\right]\right)\right.$, $\left.\operatorname{Spec}\left(\overline{\mathbb{Q}}_{p}\right)\right) \rightarrow \Xi$, with $\mathcal{O}\left[u^{ \pm 1}\right] \rightarrow \overline{\mathbb{Q}}_{p}$ given by $u \mapsto p$, i.e. to a $\Xi$-torsor over $\mathcal{O}\left[u^{ \pm 1}\right]$. (This specializes to the above $\Xi$-torsor over $K$ after $u \mapsto p$.) This now gives a quasi-split reductive group scheme $\underline{G}$ over $\mathcal{O}\left[u^{ \pm 1}\right]$ which can be described explicitly as follows: Our choice of pinning $\left(T_{H}, B_{H}, e\right)$ of $H$ identifies $\Xi$ with the group of automorphisms of $H$ that respect the pinning. Now there is an $K$-isomorphism

$$
\psi: G \stackrel{\sim}{\longrightarrow}\left(\operatorname{Res}_{\tilde{K} / K}\left(H \otimes_{\mathcal{O}} \tilde{K}\right)\right)^{\Gamma}
$$

where $\gamma \in \Gamma$ acts on the right hand side via $\xi(\gamma) \otimes \gamma$. Set

$$
\underline{G}=\left(\operatorname{Res}_{\mathcal{O}_{0}\left[v^{ \pm 1}\right] / \mathcal{O}\left[u^{ \pm 1}\right]}\left(H \otimes_{\mathcal{O}\left[u^{ \pm 1}\right]} \mathcal{O}_{0}\left[v^{ \pm 1}\right]\right)^{\Gamma}\right.
$$

where $\mathcal{O}_{0}\left[v^{ \pm 1}\right] / \mathcal{O}\left[u^{ \pm 1}\right]$ is the $\Gamma$-cover which is described in [59, 3.2] and which specializes to $\tilde{K} / K$ after base changing by $u \mapsto p$.

Now for $x$ in the building $\mathcal{B}(G, K)$ pick the torus $S$ so that $x$ is in the apartment $A(G, S, K)$ of $S$; the construction in [59, Theorem 4.1] gives a smooth affine group scheme $\underline{\mathcal{G}}$ over $\mathcal{O}[u]$ that extends $\underline{G}$ and specializes to $\mathcal{G}$ after base changing by $\mathcal{O}[u] \rightarrow K, u \mapsto p$. Let $\kappa$ denote either $K$ or $k$. Then, [59, 4.1] provides an identification of the apartment $A(G, S, K)$ in $\mathcal{B}(G, K)$ with an apartment in the building $\mathcal{B}\left(\underline{G} \otimes_{\mathcal{O}\left[u^{ \pm 1}\right]} \kappa((u)), \kappa((u))\right)$ of the group $\underline{G}_{\kappa((u))}:=\underline{G} \otimes_{\mathcal{O}\left[u^{ \pm 1}\right]} \kappa((u))$; here $\kappa((u))$ is considered as a discretely valued field with uniformizer $u$ and residue field $\kappa$. Then $\underline{\mathcal{G}} \otimes_{\mathcal{O}[u]} \kappa \llbracket u \rrbracket$ is the parahoric group scheme over $\kappa \llbracket u \rrbracket$ which is the connected stabilizer of the point $x_{\kappa((u))}$ corresponding to $x$ under this identification.

\subsection{Local models and central extensions.}

2.2.1. The results of this paragraph will be used only in 4.6 We start with the following:

Proposition 2.2.2. Suppose that $\alpha: G_{1} \rightarrow G_{2}$ is a central extension of reductive groups over $\mathbb{Q}_{p}$ and let $x_{1} \in \mathcal{B}\left(G_{1}, \mathbb{Q}_{p}\right), x_{2}=\alpha_{*}\left(x_{1}\right) \in \mathcal{B}\left(G_{2}, \mathbb{Q}_{p}\right)$. Assume that $G_{1}, G_{2}$ split over a tamely ramified extension of $\mathbb{Q}_{p}$ and denote by $\mathcal{G}_{i}, i=1,2$, the corresponding parahoric group scheme $\mathcal{G}_{x_{i}}^{\circ}$ over $\operatorname{Spec}\left(\mathbb{Z}_{p}\right)$. The group scheme homomorphism $\alpha: \mathcal{G}_{1} \rightarrow \mathcal{G}_{2}$ extends to a group scheme homomorphism $\underline{\alpha}: \underline{\mathcal{G}}_{1} \rightarrow \underline{\mathcal{G}}_{2}$ over $X=\operatorname{Spec}\left(\mathbb{Z}_{p}[u]\right)$. This gives $\underline{\alpha}_{*}: \mathrm{Gr}_{\underline{\mathcal{G}}_{1}, X} \rightarrow \mathrm{Gr}_{\underline{\mathcal{G}}_{2}, X}$ and by specializing at $u=p$, we obtain a morphism $\alpha_{*}: \mathrm{Gr}_{\underline{\mathcal{G}}_{1}, \mathbb{Z}_{p}} \rightarrow \mathrm{Gr}_{\underline{\mathcal{G}}_{2}, \mathbb{Z}_{p}}$.

Proof. We will use the notations and constructions of [59, §2, 3, 4]. Suppose that $H_{i}$ are the split forms of $G_{i}$ over $\mathbb{Z}_{p}$; we can choose pinnings $\left(T_{i}, B_{i}, \underline{e}_{i}\right)$ of $H_{i}$ and a central isogeny $\beta: H_{1} \rightarrow H_{2}$ that respects the pinnings in the sense that we have $\beta\left(T_{1}\right) \subset T_{2}, \beta\left(B_{1}\right) \subset B_{2}, \beta\left(\underline{e}_{1}\right)=\underline{e}_{2}$. Let $Z_{0} \subset T_{1}$ be the kernel of $\beta$. The quasi-split form $G_{1}^{\text {qs }}$ is given by a group homomorphism $\Gamma \rightarrow \Xi_{1}$ whose image lies in the subgroup $\Xi_{1}^{\prime} \subset \Xi_{1}$ that preserves $Z_{0}$. We have $\Xi_{1}^{\prime} \rightarrow \Xi_{2}$ and this gives $\Gamma \rightarrow \Xi_{2}$ which defines the quasi-split form $G_{2}^{\mathrm{qs}}$ together with a central $\alpha^{\mathrm{qs}}: G_{1}^{\mathrm{qs}} \rightarrow G_{2}^{\mathrm{qs}}$. The construction of the quasi-split groups $\underline{G}_{i}^{\mathrm{qs}}$ over $\operatorname{Spec}\left(\mathbb{Z}_{p}\left[u^{ \pm 1}\right]\right)$ in loc. cit., see 
also above, shows that $\alpha^{\mathrm{qs}}$ extends to a central isogeny $\underline{\alpha}^{\mathrm{qs}}: \underline{G}_{1}^{\mathrm{qs}} \rightarrow \underline{G}_{2}^{\mathrm{qs}}$. To obtain $\underline{G}_{i}$ over $\operatorname{Spec}\left(\mathbb{Z}_{p}\left[u^{ \pm 1}\right]\right)$, we set for an $\mathbb{Z}_{p}\left[u^{ \pm 1}\right]$-algebra $R$ (see $\left.[59,3.3 .4]\right)$

$$
\underline{G}_{i}(R)=\left(\underline{G}^{\mathrm{qs}}\left(\mathbb{Z}_{p}^{\mathrm{ur}}\left[u^{ \pm 1}\right] \otimes_{\mathbb{Z}_{p}\left[u^{ \pm 1}\right]} R\right)\right)^{\operatorname{Gal}\left(\mathbb{Z}_{p}^{\mathrm{ur}} / \mathbb{Z}_{p}\right)}
$$

where the action of Frobenius $\sigma \in \operatorname{Gal}\left(\mathbb{Z}_{p}^{\mathrm{ur}} / \mathbb{Z}_{p}\right)$ is given by $\operatorname{Int}\left(\mathbf{g}_{i}\right) \cdot \sigma$ with $\operatorname{Int}\left(\mathbf{g}_{i}\right)$ a certain element $\underline{G}_{i, \text { ad }}^{\mathrm{qs}}\left(\mathbb{Z}_{p}^{\mathrm{ur}}\left[u^{ \pm 1}\right]\right)$. Using $G_{1, \text { ad }}^{\mathrm{qs}}=G_{2 \text {,ad }}^{\mathrm{qs}}$, we can see that we obtain a central isogeny $\underline{\alpha}: \underline{G}_{1} \rightarrow \underline{G}_{2}$ over $\operatorname{Spec}\left(\mathbb{Z}_{p}\left[u^{ \pm 1}\right]\right)$. It remains to see that $\underline{\alpha}$ extends to a group scheme homomorphism $\underline{\alpha}: \underline{\mathcal{G}}_{1} \rightarrow \underline{\mathcal{G}}_{2}$ between the parahoric group schemes $\underline{\mathcal{G}}_{i}$ over $X=\operatorname{Spec}\left(\mathbb{Z}_{p}[u]\right)$. As in [59, 4.2.1], it is enough to show that the base change $\underline{\alpha} \otimes_{\mathbb{Z}_{p}\left[u^{ \pm 1}\right]} \mathbb{Q}_{p}((u))$ extends to a group homomorphism between

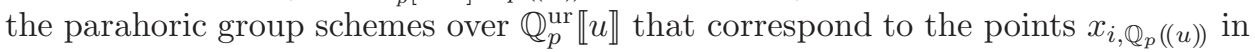
the building of $\underline{G}\left(\mathbb{Q}_{p}((u))\right)$ that correspond to $x_{i}$, as in [59, 4.1.3]; this then follows from the construction in loc. cit.. The rest then follows from this and the definition of the affine Grassmannians $\mathrm{Gr}_{\underline{\mathcal{G}}, X}$ in [59, 6.2].

2.2.3. Suppose $G$ is a reductive group over $\mathbb{Q}_{p}$ which splits over a tamely ramified extension, and denote by ad $: G \rightarrow G^{\text {ad }}$ the natural homomorphism. If $x \in \mathcal{B}\left(G, \mathbb{Q}_{p}\right)$ with $\bar{x}=\operatorname{ad}_{*}(x) \in \mathcal{B}\left(G^{\text {ad }}, \mathbb{Q}_{p}\right)$, we have a morphism

$$
\operatorname{ad}_{*}: \mathrm{M}_{G,\{\mu\}, x}^{\mathrm{loc}} \rightarrow \mathrm{M}_{G^{\text {ad }},\left\{\mu_{\mathrm{ad}}\right\}, \bar{x}}^{\mathrm{loc}} \otimes \mathcal{O}_{E_{\text {ad }}} \mathcal{O}_{E}
$$

which is given using the definition of the local model and Proposition 2.2.2 applied to ad : $G \rightarrow G^{\text {ad }}$. For simplicity, we will denote the parahoric group scheme for $G$ that corresponds to $\bar{x}$ by $\mathcal{G}$, we will also use $\mathcal{G}^{\text {ad }}$, resp. $\mathcal{G}^{\text {der }}$, for the corresponding parahoric group schemes for $G^{\text {ad }}$, resp. $G^{\text {der }}$.

Proposition 2.2.4. Assume $\pi_{1}\left(G^{\text {der }}\right)$ has order prime to $p$. Then the morphism $\operatorname{ad}_{*}$ induces an isomorphism

$$
\operatorname{ad}_{*}^{\sim}: \mathrm{M}_{G,\{\mu\}, x}^{\mathrm{loc}} \stackrel{\sim}{\longrightarrow}\left(\mathrm{M}_{G^{\mathrm{ad}},\left\{\mu_{\mathrm{ad}}\right\}, \bar{x}}^{\mathrm{loc}} \otimes \mathcal{O}_{E_{\mathrm{ad}}} \mathcal{O}_{E}\right)^{\sim}
$$

where the target is the normalization of the base change of $\mathrm{M}_{G^{\mathrm{ad}},\left\{\mu_{\mathrm{ad}}\right\}, \bar{x}}^{\mathrm{loc}}$. The isomorphism $\operatorname{ad}_{*}^{\sim}$ is equivariant with respect to ad $: \mathcal{G} \rightarrow \mathcal{G}^{\text {ad }}$ and hence, the natural action of $\mathcal{G}$ on $\mathrm{M}_{G,\{\mu\}, x}^{\mathrm{loc}}$ factors through an action of $\mathcal{G}^{\mathrm{ad}}$.

Proof. Since $\mathcal{G}^{\text {ad }}$ is smooth, the natural action of $\mathcal{G}^{\text {ad }}$ on $\mathrm{M}_{G^{\text {ad }},\left\{\mu_{\text {ad }}\right\}, \bar{x}}^{\text {loc }}$ extends to the normalization of the base change. By the definitions, the $\operatorname{morphism} \mathrm{ad}_{*}$ is equivariant with respect to ad $: \mathcal{G} \rightarrow \mathcal{G}^{\text {ad }}$. Since by Theorem 2.1.2. $\mathrm{M}_{G,\{\mu\}, x}^{\mathrm{loc}}$ is normal, $\mathrm{ad}_{*}$ induces a morphism $\mathrm{ad}_{*}^{\sim}$ as above which is also equivariant. From the definition, one sees that $\operatorname{ad}_{*} \otimes_{\mathcal{O}_{E}} E$ is an isomorphism. Using [59, Cor. 6.6], we see that the morphism $\operatorname{ad}_{*} \otimes_{\mathcal{O}_{E}} k$ is given by restricting the corresponding natural morphism $\operatorname{Gr}_{P_{k}} \rightarrow \operatorname{Gr}_{P_{k}}$ of affine Grassmannians. Here the group schemes $P_{k}=\underline{\mathcal{G}} \otimes_{\mathbb{Z}_{p}[u]} k \llbracket u \rrbracket$ and $P_{k}^{\text {ad }}=\underline{\mathcal{G}}^{\text {ad }} \otimes_{\mathbb{Z}_{p}[u]} k \llbracket u \rrbracket$ are as in loc. cit.. We can now see that the induced morphism from each connected component of $\mathrm{Gr}_{P_{k}}$ to $\mathrm{Gr}_{P_{k}}$ ga gives a finite to one map on $k$-valued points. (See [57, $\S 6$ (a), (b)], especially the proof of $(6.19)$ there). Hence, the restriction $\operatorname{ad}_{*} \otimes_{\mathcal{O}_{E}} k$ is quasi-finite. Since both its source and target are normal and proper, it follows, using Zariski's main theorem, that $\mathrm{ad}_{*}^{\sim}$ is an isomorphism.

2.2.5. We assume that we have two triples $(G,\{\mu\}, \mathrm{K}),\left(G^{\prime},\left\{\mu^{\prime}\right\}, \mathrm{K}^{\prime}\right)$, over $K=\mathbb{Q}_{p}$ as in 2.1.1 that, in addition, satisfy the following: 
a) There is a central isogeny $\alpha: G^{\text {der }} \rightarrow G^{\prime \text { der }}$ which induces an isomorphism $\alpha^{\text {ad }}:\left(G^{\text {ad }},\left\{\mu_{\mathrm{ad}}\right\}\right) \stackrel{\sim}{\rightarrow}\left(G^{\text {ad }},\left\{\mu_{\mathrm{ad}}^{\prime}\right\}\right)$,

b) The parahoric subgroups $\mathrm{K} \subset G\left(\mathbb{Q}_{p}\right), \mathrm{K}^{\prime} \subset G^{\prime}\left(\mathbb{Q}_{p}\right)$, correspond to points $x \in \mathcal{B}\left(G, \mathbb{Q}_{p}\right), x^{\prime} \in \mathcal{B}\left(G^{\prime}, \mathbb{Q}_{p}\right)$, that map to the same point $\bar{x}$ in $\mathcal{B}\left(G^{\text {ad }}, \mathbb{Q}_{p}\right)=$ $\mathcal{B}\left(G^{\text {ad }}, \mathbb{Q}_{p}\right)$, where the identification is via $\alpha^{\text {ad }}$ as in (a),

c) The prime $p$ does not divide the order of $\pi_{1}\left(G^{\prime \text { der }}\right)$.

Under the assumptions (a)-(c), we will compare the local models $\mathrm{M}_{G,\{\mu\}, x}^{\mathrm{loc}}$ and $\mathrm{M}_{G^{\prime},\left\{\mu^{\prime}\right\}, x^{\prime}}^{\text {loc }}$ Let $E$, resp. $E^{\prime}$, the reflex field of $(G,\{\mu\})$, resp. $\left(G^{\prime},\left\{\mu^{\prime}\right\}\right)$, and denote by $E_{\text {ad }}$ the reflex field of $\left(G^{\text {ad }},\left\{\mu_{\text {ad }}\right\}\right)$. Using (a) above, we obtain $E_{\text {ad }} \subset E, E^{\prime}$.

Denote by $C$ the kernel of $\alpha$. By (c), $C$ is a finite group scheme of rank prime to $p$. For simplicity, we will denote the parahoric group schemes that correspond to $\bar{x}$ by $\mathcal{G}, \mathcal{G}^{\prime}$, etc. The central isogeny extends to a group scheme homomorphism $\alpha: \mathcal{G}^{\text {der }} \rightarrow \mathcal{G}^{\prime \text { der }}$. We have $\mathcal{G}^{\text {ad }}=\mathcal{G}^{\text {ad }}$, and by Proposition 1.1.4,

$$
\mathcal{G}^{\prime \text { der }} \simeq \mathcal{G}^{\text {der }} / \mathcal{C}
$$

where $\mathcal{C}$ is the (smooth) schematic closure of $C$ in $\mathcal{G}^{\text {der }}$ and the isomorphism is induced by $\alpha$.

Proposition 2.2.7. Under the assumptions (a)-(c), there is an isomorphism

$$
\mathrm{M}_{G,\{\mu\}, x}^{\text {loc }} \otimes_{\mathcal{O}_{E}} \mathcal{O}_{E E^{\prime}} \stackrel{\sim}{\longrightarrow} \mathrm{M}_{G^{\prime},\left\{\mu^{\prime}\right\}, x^{\prime}}^{\text {loc }} \otimes_{\mathcal{O}_{E^{\prime}}} \mathcal{O}_{E E^{\prime}}
$$

which is equivariant with respect to $\alpha: \mathcal{G}^{\text {der }} \rightarrow \mathcal{G}^{\prime \text { der }}$.

Here the source, resp. target, of the isomorphism admits an action of the group scheme $\mathcal{G}^{\text {der }}$, resp. $\mathcal{G}^{\prime \text { der }}$, by restricting the natural action of $\mathcal{G}$, resp. of $\mathcal{G}^{\prime}$.

Proof. Under the assumptions (a)-(c), the order of $\pi_{1}\left(G^{\text {der }}\right) \subset \pi_{1}\left(G^{\prime \text { der }}\right)$ is also prime to $p$. Hence, Proposition 2.2.4 applies to both $G$ and $G^{\prime}$ to produce isomorphisms $\operatorname{ad}_{*}^{\sim}, \operatorname{ad}_{*}^{\prime \sim}$. Consider now

$$
\left(\operatorname{ad}_{*}^{\prime \sim}\right)^{-1} \cdot \tau \cdot \tilde{a d}_{*}: \mathrm{M}_{G,\{\mu\}, x}^{\mathrm{loc}} \otimes_{\mathcal{O}_{E}} \mathcal{O}_{E E^{\prime}} \stackrel{\sim}{\rightarrow} \mathrm{M}_{G^{\prime},\left\{\mu^{\prime}\right\}, x^{\prime}}^{\mathrm{loc}} \otimes_{\mathcal{O}_{E^{\prime}}} \mathcal{O}_{E E^{\prime}} .
$$

Here we use the natural isomorphism

$$
\tau:\left(M_{\mathrm{ad}} \otimes_{\mathcal{O}_{E_{\mathrm{ad}}}} \mathcal{O}_{E}\right)^{\sim} \otimes_{\mathcal{O}_{E}} \mathcal{O}_{E E^{\prime}} \stackrel{\sim}{\rightarrow}\left(M_{\mathrm{ad}} \otimes_{\mathcal{O}_{E_{\mathrm{ad}}}} \mathcal{O}_{E^{\prime}}\right)^{\sim} \otimes_{\mathcal{O}_{E^{\prime}}} \mathcal{O}_{E E^{\prime}}
$$

which exists since, by Corollary 2.1.3 both its source and target are normal, and therefore agree with the normalization of $M_{\mathrm{ad}} \otimes_{\mathcal{O}_{E \mathrm{ad}}} \mathcal{O}_{E E^{\prime}}$. (In this we set $M_{\mathrm{ad}}=$ $\mathrm{M}_{G^{\text {ad }},\left\{\mu_{\mathrm{ad}}\right\}, \bar{x}}^{\mathrm{loc}}$ for simplicity.) It remains to show the claimed equivariance property. Using flatness, we see that it is enough to check this on the generic fibres; there it follows easily from the definitions.

\subsection{Embedding local models in Grassmannians.}

2.3.1. Here we assume that $K=\mathbb{Q}_{p}$ and that $(G,\{\mu\}, \mathrm{K})$ is as above. Suppose we also have a faithful symplectic representation $\rho: G \rightarrow \operatorname{GSp}(V) \subset \operatorname{GL}(V)$. We suppose that the composite $\rho \cdot \mu$ is conjugate to the minuscule coweight $\mu_{0}$ of $\operatorname{GSp}(V)$ given by $a \mapsto \operatorname{diag}\left(a^{(g)}, 1^{(g)}\right)$ and that the symplectic representation $\rho$ is minuscule (cf. table 20] 1.3.9). We also assume that $G \subset \mathrm{GL}(V)$ contains the diagonal torus $\mathbb{G}_{\mathrm{m}}$ of scalars. We will call such a $\rho$ a (local) Hodge embedding.

Choose an $\mathbb{Q}_{p}$-split torus $A$ such that $x \in A\left(G, A, \mathbb{Q}_{p}\right) \subset \mathcal{B}\left(G, \mathbb{Q}_{p}\right)$; choose also a maximal $\mathbb{Q}_{p}^{\text {ur }}$-split torus $S$ in $G$ that contains $A$ and is defined over $\mathbb{Q}_{p}$ (such a 
torus exists by [11, 5.1.12]); since $G_{\mathbb{Q}_{p}^{\text {ur }}}$ is quasi-split, $T=Z_{G}(S)$ is a maximal torus of $G$ which is defined over $\mathbb{Q}_{p}$ and splits over $\tilde{K}$. Suppose we also choose a pinning $\left(T_{H}, B_{H}, \underline{e}\right)$ of the split Chevalley form $H$ of $G$ over $\mathbb{Z}_{p}$. Again, since $G$ splits over $\tilde{K}$ and $G_{\mathbb{Q}_{p}^{\text {ur }}}$ is quasi-split, we can choose $\psi: G_{\tilde{K}} \stackrel{\sim}{\rightarrow} H_{\tilde{K}}$ that maps $T_{\tilde{K}}$ to $\left(T_{H}\right)_{\tilde{K}}$ and is such that the Borel subgroup $\psi^{-1}\left(\left(B_{H}\right)_{\tilde{K}}\right) \subset G_{\tilde{K}}$ is defined over $\mathbb{Q}_{p}^{\text {ur }}$. Then for $\gamma$ in the inertia $I_{\tilde{K}}=\operatorname{Gal}\left(\tilde{K} /\left(\tilde{K} \cap \mathbb{Q}_{p}^{\text {ur }}\right)\right), c(\gamma):=\psi \cdot \gamma(\psi)^{-1} \in \operatorname{Aut}(H)(\tilde{K})$ preserves $\left(T_{H}\right)_{\tilde{K}}$ and $\left(B_{H}\right)_{\tilde{K}}$. Furthermore, by composing $\psi$ with the (conjugation) action of an element of $T_{H \text { ad }}(\tilde{K})$ we can suppose that $c(\gamma)$ is a diagram automorphism, i.e. it preserves the pinning $\left(T_{H}, B_{H}, \underline{e}\right) \times_{\mathbb{Z}_{p}} \tilde{K}$. Recall now that starting with the pinning $\left(T_{H}, B_{H}, \underline{e}\right)$ of $H$, the isomorphism $\psi$, the choice of irreducible summands $V_{j}$, and for each such summand, the choice of a highest weight vector $v_{1}$ and the lattice chain gradings given by the translations $t \in \mathbb{R}$, we have constructed in the previous paragraph a $G\left(\mathbb{Q}_{p}^{\text {ur }}\right)$-equivariant toral embedding

$$
\iota: \mathcal{B}\left(G, \mathbb{Q}_{p}^{\text {ur }}\right) \rightarrow \mathcal{B}\left(\mathrm{GL}(V), \mathbb{Q}_{p}^{\text {ur }}\right)
$$

which is also $\operatorname{Gal}\left(\mathbb{Q}_{p}^{\text {ur }} / \mathbb{Q}_{p}\right)$-equivariant. Note that there is also a canonical equivariant toral embedding

$$
s: \mathcal{B}\left(\mathrm{GSp}(V), \mathbb{Q}_{p}^{\text {ur }}\right) \rightarrow \mathcal{B}\left(\mathrm{GL}(V), \mathbb{Q}_{p}^{\text {ur }}\right) .
$$

Lemma 2.3.3. There is a choice of the above data such that $\iota$ factors

$$
\mathcal{B}\left(G, \mathbb{Q}_{p}^{\text {ur }}\right) \stackrel{j}{\rightarrow} \mathcal{B}\left(\mathrm{GSp}(V), \mathbb{Q}_{p}^{\text {ur }}\right) \stackrel{s}{\rightarrow} \mathcal{B}\left(\mathrm{GL}(V), \mathbb{Q}_{p}^{\text {ur }}\right) .
$$

Proof. We will use results of Satake [66] on symplectic representations. Consider the similitude character $\chi: G \subset \mathrm{GL}(V) \rightarrow \mathbb{G}_{\mathrm{m}}$ and denote by $G_{1} \subset G$ its kernel so that $\rho\left(G_{1}\right) \subset \operatorname{Sp}(V)$. We have $\mathcal{B}\left(G, \mathbb{Q}_{p}^{\text {ur }}\right)=\mathcal{B}\left(G_{1}, \mathbb{Q}_{p}^{\text {ur }}\right) \times \mathbb{R}, \mathcal{B}\left(\operatorname{GSp}(V), \mathbb{Q}_{p}^{\text {ur }}\right)=$ $\mathcal{B}\left(\operatorname{Sp}(V), \mathbb{Q}_{p}^{\text {ur }}\right) \times \mathbb{R}$ and we can see that it is enough to show that there is a choice of data as above such that the corresponding $\iota$ maps $\mathcal{B}\left(G_{1}, \mathbb{Q}_{p}^{\text {ur }}\right)$ to $\mathcal{B}\left(\operatorname{Sp}(V), \mathbb{Q}_{p}^{\text {ur }}\right)$. Following [66], we canonically decompose $V=\oplus_{a} V_{a}$ as the direct sum of its $\mathbb{Q}_{p^{-}}$ primary $G$-summands. (Recall that a $G$-representation $W$ is called $\mathbb{Q}_{p}$-primary when for every two absolutely irreducible $G$-summands $W_{1}$ and $W_{2}$ of $W \otimes_{\mathbb{Q}_{p}} \overline{\mathbb{Q}}_{p}$, there is $\sigma \in \operatorname{Gal}\left(\overline{\mathbb{Q}}_{p} / \mathbb{Q}_{p}\right)$ such that $W_{1} \simeq \sigma\left(W_{2}\right)$ as $G$-representations.) As in loc. cit., there are three different types of $\mathbb{Q}_{p}$-primary components of $V$ that can be distinguished as follows. If $W_{a}$ is a geometrically irreducible $G_{1}$-summand of $V_{a} \otimes_{\mathbb{Q}_{p}} \overline{\mathbb{Q}}_{p}$ we have:

a) $W_{a}^{\vee} \simeq W_{a}$

b) $W_{a}^{\vee} \simeq \sigma\left(W_{a}\right)$, for some $\sigma \in \operatorname{Gal}\left(\overline{\mathbb{Q}}_{p} / \mathbb{Q}_{p}\right)$, or,

c) $W_{a}^{\vee} \not \sigma\left(W_{a}\right)$, for all $\sigma \in \operatorname{Gal}\left(\overline{\mathbb{Q}}_{p} / \mathbb{Q}_{p}\right)$.

Using our construction of $\iota$ and the discussion in [66, 2.1] we can easily reduce to the case that either $V=V_{a}$ is $\mathbb{Q}_{p}$-primary and of type (a) or (b), or $V=V_{a} \oplus V_{a}^{\vee}$ with $V_{a}$ of type (c). Assume that $V=V_{a}$ and is of type (a). Note that $V$ here does not have to be $\mathbb{Q}_{p}$-irreducible but we can write $V=V^{\prime \oplus m}$ where $V^{\prime}$ is $\mathbb{Q}_{p^{-}}$ irreducible. The set-up of 1.2 .15 applies to the $\mathbb{Q}_{p}$-irreducible $G_{1}$-representation $V^{\prime}$. By [66, Theorem 1] (using the notation of 1.2 .15 for $V^{\prime}$ ) we see that there is a field extension $K_{1} / K$, a central division algebra $D$ over $K_{1}$ with an involution of the first kind, a right $D$-module $V_{1}^{\prime}$ and a left $D$-module $V_{2}$, with $m=\operatorname{dim}_{D}\left(V_{2}\right)$, together with a non-degenerate $\varepsilon$-hermitian, resp. $(-\varepsilon)$-hermitian, form $h_{1}$ on $V_{1}^{\prime}$, 
resp. $h_{2}$ on $V_{2}$, such that the following is true: The restriction of $\rho$ to $G_{1}$ factors as the composition of

with the restriction of scalars of

$$
G_{1} \rightarrow \operatorname{Res}_{K_{1} / K}\left(G_{1, K_{1}}\right)
$$

$$
G_{1, K_{1}} \stackrel{\left(\rho_{1}, 1\right)}{\longrightarrow} \mathrm{U}\left(V_{1}^{\prime} / D, h_{1}\right) \times \mathrm{U}\left(D \backslash V_{2}, h_{2}\right) \stackrel{\otimes}{\longrightarrow} \mathrm{Sp}\left(V_{1}\right) .
$$

and the natural $\operatorname{Res}_{K_{1} / K}\left(\operatorname{Sp}\left(V_{1}\right)\right) \rightarrow \operatorname{Sp}(V)$. (Here, $V_{1}=V_{1}^{\prime} \otimes_{D} V_{2}$ is $V$ which has a $K_{1}$-module structure; $V_{1}$ supports a non-degenerate alternating form given via the $D / K_{1}$ trace of the tensor product of $h_{1}$ and ${ }^{t} h_{2}$, see loc. cit.. Recall that $V_{1}^{\prime} \otimes_{K_{1}} \bar{K}$ is an irreducible Weyl module.) By the main result of [48] there exists an equivariant toral map $\iota_{1}^{\prime}: \mathcal{B}\left(G_{1}, K_{1}^{\text {ur }}\right) \rightarrow \mathcal{B}\left(\mathrm{U}\left(V_{1}^{\prime} / D, h_{1}\right), K_{1}^{\text {ur }}\right)$; in fact, this is obtained by taking $\operatorname{Gal}\left(\tilde{K} / K_{1}^{\text {ur }}\right)$-fixed points of a $\operatorname{Gal}\left(\tilde{K} / K_{1}^{\text {ur }}\right)$-equivariant toral map $\iota_{1}^{\prime}(\tilde{K}): \mathcal{B}\left(G_{1}, \tilde{K}\right) \rightarrow \mathcal{B}\left(\mathrm{U}\left(V_{1}^{\prime} / D, h_{1}\right), \tilde{K}\right)$. Using the uniqueness argument in the proof of Proposition 1.2 .21 we see that the map $\iota_{1}^{\prime}$, when composed with $\mathcal{B}\left(\mathrm{U}\left(V_{1}^{\prime} / D, h_{1}\right), K_{1}^{\mathrm{ur}}\right) \subset \mathcal{B}\left(\mathrm{GL}_{D}\left(V_{1}^{\prime}\right), K_{1}^{\text {ur }}\right)$ agrees with $t+\iota_{1}$ as in (1.2.23), for a suitable choice of translation $t$. The result now follows from the construction of $\iota$ and the above. The argument for type (b) is similar. Finally, in type (c) the alternating form on $V$ is given by the duality between the Lagrangian subspaces $V_{a}$ and $V_{a}^{\vee}$ in $V$. This case is simpler and is also left to the reader.

2.3.4. For $x \in \mathcal{B}\left(G, \mathbb{Q}_{p}\right)$ as before, consider the parahoric group scheme $\mathcal{G S P}_{z}$ of $\operatorname{GSp}(V)$ that corresponds to $z=j(x)$. As before, set $y=\iota(x)$. Since $z=s(y)$, the corresponding (periodic) lattice chain $\Lambda_{y}^{\bullet}$ is self-dual. We have affine smooth group scheme homomorphisms

$$
\rho: \mathcal{G}_{x} \rightarrow \mathcal{G S} \mathcal{P}_{z} \rightarrow \mathcal{G L}_{y}
$$

By Proposition 1.3.3. $\mathcal{G}_{x} \rightarrow \mathcal{G} \mathcal{L}_{y}$ and therefore $\mathcal{G}_{x} \rightarrow \mathcal{G S P} z$ is a closed immersion.

The corresponding local model $\mathrm{M}_{\mathrm{GSp}}^{\mathrm{loc}}:=\mathrm{M}_{\mathrm{GSp}(V),\left\{\mu_{0}\right\}, z}^{\mathrm{loc}}$ for the group $\operatorname{GSp}(V)$, its standard minuscule coweight $\mu_{0}$ and the periodic self dual lattice chain $\Lambda_{z}^{\bullet}$ that corresponds to $z$ was considered by Görtz in [31; in this case, this agrees with the corresponding local model of [59] as explained in loc. cit.. The generic fibre of $\mathrm{M}_{\mathrm{GS}}^{\mathrm{loc}}$ over $\mathbb{Q}_{p}$ is the Lagrangian Grassmannian $\operatorname{LGr}(V)$ of maximal isotropic subspaces in $V$. The standard embedding $\mathcal{G S P} \mathcal{P}_{z} \rightarrow \mathcal{G} \mathcal{L}_{y}$ induces a closed immersion $\mathrm{M}_{\mathrm{GSp}}^{\text {loc }} \hookrightarrow$ $\mathrm{M}_{\mathrm{GL}(V),\left\{\mu_{0}\right\}, y}^{\mathrm{loc}}$. Since the composition of $\mu$ with $\rho$ is conjugate to the standard minuscule coweight $\mu_{0}$ of $\operatorname{GSp}(V)$ the embedding $\rho$ induces a closed immersion

$$
X_{\mu} \hookrightarrow \operatorname{LGr}(V) \otimes_{\mathbb{Q}_{p}} E .
$$

Proposition 2.3.7. With the above assumptions and notations, 2.3.6) extends to a closed immersion

$$
\mathrm{M}_{G}^{\mathrm{loc}}=\mathrm{M}_{G,\{\mu\}, x}^{\mathrm{loc}} \hookrightarrow \mathrm{M}_{\mathrm{GSp}(V),\left\{\mu_{0}\right\}, z}^{\mathrm{loc}} \otimes_{\mathbb{Z}_{p}} \mathcal{O}_{E} .
$$

Proof. For simplicity, we set $L=\mathbb{Q}_{p}^{\text {ur }}$ and $E^{\prime}=E L$. It is enough to show that the base change (2.3.6) $\times_{E} E^{\prime}$ extends to a closed immersion

$$
\mathrm{M}_{G,\{\mu\}, x}^{\mathrm{loc}} \otimes_{\mathcal{O}_{E}} \mathcal{O}_{E^{\prime}} \hookrightarrow \mathrm{M}_{\mathrm{GL}(V),\left\{\mu_{0}\right\}, y}^{\mathrm{loc}} \otimes_{\mathbb{Z}_{p}} \mathcal{O}_{E^{\prime}}
$$

Indeed, assuming this, we easily verify that (2.3.9) descends over $\mathcal{O}_{E}$ by checking the descent condition on the generic fibre.

Now recall that, by construction ([59]), we have $\mathrm{M}_{G,\{\mu\}, x}^{\text {loc }} \otimes_{\mathcal{O}_{E}} \mathcal{O}_{E^{\prime}}=\mathrm{M}_{G_{L},\{\mu\}, x}^{\mathrm{loc}}$, where $\mathrm{M}_{G_{L},\{\mu\}, x}^{\mathrm{loc}}$ is the local model for the triple $\left(G_{L},\{\mu\}, x\right)$ over $L$. (Here, we 
use the obvious extension of the definition of local models over $L=\mathbb{Q}_{p}^{\text {ur }}$.) Using the above we now see that it will be enough to show the closed immersion claim for the local model over $\mathcal{O}_{E^{\prime}}$ associated to the triple $\left(G_{L},\{\mu\}, x\right)$ and the (faithful) representation $\rho_{L}:=\rho \otimes_{\mathbb{Q}_{p}} L: G_{L} \hookrightarrow \mathrm{GL}\left(V_{L}\right)$ over $L$ obtained by base change.

As in 1.2. write $\rho=\prod_{j} \rho_{j}$ with $\rho_{j}: G \rightarrow \mathrm{GL}\left(V_{j}\right)$ irreducible over $\mathbb{Q}_{p}$. We return to the set-up of 1.2 .15 for $\rho_{j}$ over the base field $\mathbb{Q}_{p}$; we can choose the field $\tilde{\mathbb{Q}}_{p}$ there so that $L \subset \tilde{\mathbb{Q}}_{p}$. We have representations

$$
\rho_{j 1, \tilde{\mathbb{Q}}_{p}}: G_{\tilde{\mathbb{Q}}_{p}} \cong H_{\tilde{\mathbb{Q}}_{p}} \rightarrow \mathrm{GL}\left(V\left(\lambda_{j 1}\right) \otimes_{\mathbb{Q}_{p}} \tilde{\mathbb{Q}}_{p}\right) \text {. }
$$

Let $\Gamma_{j 1}$ be the subgroup of $\operatorname{Gal}\left(\tilde{\mathbb{Q}}_{p} / \mathbb{Q}_{p}\right)$ fixing the weight $\lambda_{j 1}$ and $\mathbb{Q}_{p, j 1}$ the corresponding subfield of $\tilde{\mathbb{Q}}_{p}$. Set $I_{j 1}$ for the subgroup of $\Gamma_{j 1}$ with fixed field $L \mathbb{Q}_{p, j 1}$. For simplicity, set $L_{j}=L \mathbb{Q}_{p, j 1} \supset L$. After taking fixed points, i.e. descending, by the action of $I_{j 1}$ described in 1.2 .15 we obtain

$$
\rho_{j 1, L_{j}}: G_{L_{j}} \cong\left(H_{\tilde{\mathbb{Q}}_{p}}\right)^{I_{j 1}} \rightarrow\left(\mathrm{GL}\left(V\left(\lambda_{j 1}\right) \otimes_{\mathbb{Q}_{p}} \tilde{\mathbb{Q}}_{p}\right)\right)^{I_{j 1}} .
$$

Recall that $G_{L}$ is quasi-split by Steinberg's theorem. In fact, we can assume that the action of $I_{j 1}$ preserves the Borel subgroup $\psi^{-1}\left(\left(B_{H}\right)_{\tilde{\mathbb{Q}}_{p}}\right)$. Then the argument in the proof of Theorem 3.3 in [69] shows that the group $I_{j 1}$ acts via a cocycle $I_{j 1} \rightarrow \operatorname{GL}\left(V\left(\lambda_{j 1}\right) \otimes_{\mathbb{Q}_{p}} \tilde{\mathbb{Q}}_{p}\right)$ which lifts the cocycle $c^{\prime}$ of 1.2 .15 (see also Step 1 below). This allows us to view $\rho_{j 1, L_{j 1}}$ as a representation

$$
\rho_{j, L_{j}}^{\prime}: G_{L_{j}} \rightarrow \mathrm{GL}\left(V_{j}^{\prime}\right)
$$

where $V_{j}^{\prime}=\left(V\left(\lambda_{j 1}\right) \otimes_{\mathbb{Q}_{p}} \tilde{\mathbb{Q}}_{p}\right)^{I_{j 1}}$ is a $L_{j}$-vector space such that $V_{j}^{\prime} \otimes_{L_{j}} \tilde{\mathbb{Q}}_{p} \cong$ $V\left(\lambda_{j 1}\right) \otimes_{\mathbb{Q}_{p}} \tilde{\mathbb{Q}}_{p}$. Consider the composition

$$
\rho_{j, L}^{\prime}: G_{L} \rightarrow \operatorname{Res}_{L_{j} / L}\left(G_{L_{j}}\right) \stackrel{\operatorname{Res}_{L_{j} / L}\left(\rho_{j, L_{j}}^{\prime}\right)}{\longrightarrow} \operatorname{Res}_{L_{j} / L}\left(\mathrm{GL}\left(V_{j}^{\prime}\right)\right) \rightarrow \mathrm{GL}\left(V_{j, L}^{\prime}\right),
$$

where $V_{j, L}^{\prime}$ is, by definition, $V_{j}^{\prime}$ regarded as a $L$-vector space by restriction of scalars.

The base change $\rho_{L}:=\rho \otimes_{\mathbb{Q}_{p}} L$ can be identified with

$$
\rho_{L}: G_{L} \stackrel{\prod_{j} \prod_{\tau} \rho_{j, L}^{\prime}}{\longrightarrow} \prod_{j} \prod_{\tau} \mathrm{GL}\left(V_{j, L}^{\prime}\right) \subset \mathrm{GL}\left(V_{L}\right)
$$

where $V_{L}:=\oplus_{j} \oplus_{\tau} V_{j, L}^{\prime}$ and $\tau$ runs over a finite set of $\mathbb{Q}_{p}$-automorphisms $\tau: L \rightarrow L$ that depends on $j$ and is in bijection with the orbit $\left\{\tau\left(\lambda_{j 1}\right)\right\}$. As in Proposition 1.2.21 we obtain an equivariant map of buildings

$$
\iota_{j}^{\prime}: \mathcal{B}\left(G, L_{j}\right) \rightarrow \mathcal{B}\left(\mathrm{GL}\left(V_{j}^{\prime}\right), L_{j}\right)
$$

which as in 1.2 .22 produces a $G(L)$-equivariant map of buildings

$$
\iota_{j, L}: \mathcal{B}(G, L) \rightarrow \mathcal{B}\left(\mathrm{GL}\left(V_{j, L}^{\prime}\right), L\right),
$$

corresponding to $\rho_{j, L}^{\prime}: G_{L} \rightarrow \mathrm{GL}\left(V_{j, L}^{\prime}\right)$. Set $y_{j}^{\prime}:=\iota_{j, L}(x)$. The image of $\left(\tau\left(y_{j, L}^{\prime}\right)\right)_{j, \tau}$ under the natural equivariant embedding

$$
\prod_{j} \prod_{\tau} \mathcal{B}\left(\mathrm{GL}\left(V_{j, L}^{\prime}\right), L\right) \subset \mathcal{B}\left(\mathrm{GL}\left(V_{L}\right), L\right)
$$

is $y=\iota(x) \in \mathcal{B}\left(\mathrm{GL}(V), \mathbb{Q}_{p}\right) \subset \mathcal{B}\left(\mathrm{GL}\left(V_{L}\right), L\right)$.

For simplicity, we set $\mathcal{O}=\mathcal{O}_{L}=\mathcal{O}^{\text {ur }}$ and denote by $k$ the residue field of $\mathcal{O}_{L}$. Using [59, Prop. 8.1] and the above, we see that it is enough to show that $\rho_{L}$ : $G_{L} \hookrightarrow \mathrm{GL}\left(V_{L}\right)$ extends to a group scheme homomorphism $\underline{\rho}_{\mathcal{O}[u]}: \underline{\mathcal{G}} \rightarrow \mathrm{GL}\left(N_{\bullet}\right)$ over 
$\operatorname{Spec}(\mathcal{O}[u])$ (for some periodic $\mathcal{O}[u]$-lattice chain $N_{\bullet}$ ) which satisfies the following condition from loc. cit. 8.1.1:

$\left(^{*}\right)$ The Zariski closure of of $\underline{\mathcal{G}} \otimes_{\mathcal{O}[u]} k((u))$ in $\mathrm{GL}\left(N_{\bullet} \otimes_{\mathcal{O}[u]} k \llbracket u \rrbracket\right)$ is a smooth group scheme which stabilizes the point $x_{k((u))}$ and $\underline{\rho}_{\mathcal{O}[u]} \otimes_{\mathcal{O}[u]} k \llbracket u \rrbracket$ identifies the group scheme $\underline{\mathcal{G}} \otimes_{\mathcal{O}[u]} k((u))=\mathcal{G}_{x_{k((u))}}^{\circ}$ with the neutral component of that Zariski closure.

(The homomorphism $\underline{\rho}_{\mathcal{O}[u]}$ then produces a corresponding morphism between local models as in [59. Actually, [59, 8.1] discusses embeddings into group schemes related to GSp instead of GL but the argument is the same.) In fact, we will first show that, for all $j, \rho_{j, L_{j}}^{\prime}: G_{L_{j}} \rightarrow \mathrm{GL}\left(V_{j}^{\prime}\right)$, and $\rho_{j, L}^{\prime}: G_{L} \rightarrow \mathrm{GL}\left(V_{j, L}^{\prime}\right)$ as above, suitably extend. Then we will deduce that $\rho_{L}$ also extends in the desired way. We will do this in several steps:

Step 1. We first show that, for all $j, \rho_{j, L_{j}}^{\prime}$ and $\rho_{j, L}^{\prime}$ extend to representations over Laurent polynomial rings with coefficients in $\mathcal{O}$. If $e_{j}$ is the (ramification) degree of $L_{j} / L$, we consider the cover $\mathcal{O}[u] \rightarrow \mathcal{O}[v], u \mapsto v^{e_{j}}$. We identify the generic fibre of the specialization of this cover under $u \mapsto p$ with $L_{j} / L$. Recall that we start with a point $x$ in the building $\mathcal{B}\left(G, \mathbb{Q}_{p}\right) \subset \mathcal{B}(G, L)$ which lies in the apartment $A(G, S, L)$ of the $L$-split torus $S$. We have chosen a pinning $\left(T_{H}, B_{H}, \underline{e}\right)$ of the Chevalley split form $H$ of $G$ over $\mathbb{Z}_{p}$ which gives a hyperspecial point $x_{o}$ of $\mathcal{B}\left(H, \mathbb{Q}_{p}\right)$ in the apartment of the standard torus $T_{H}$. We have also chosen the isomorphism $\psi: G_{\tilde{\mathbb{Q}}_{p}} \stackrel{\sim}{\rightarrow} H_{\tilde{\mathbb{Q}}_{p}}$ as in (2.3.1). In particular, $T_{\tilde{\mathbb{Q}}_{p}}$ maps isomorphically under $\psi$ to the standard torus $\left(T_{H}\right)_{\tilde{\mathbb{Q}}_{p}}$ and, in fact, $c(\gamma)=\psi \cdot \gamma(\psi)^{-1}$ preserves the pinning, i.e. it is a diagram automorphism.

Recall that $\rho_{j 1, \tilde{\mathbb{Q}}_{p}}$ is given by a Weyl module $V\left(\lambda_{j 1}\right) \otimes_{\mathbb{Q}_{p}} \tilde{\mathbb{Q}}_{p}$ for the highest weight $\lambda_{j 1}$ of $H$. For simplicity, we will write $\lambda_{j}$ instead of $\lambda_{j 1}$. Recall we fix a vector $v_{j} \in V\left(\lambda_{j}\right)$ of highest weight $\lambda_{j}$ and consider the $\mathbb{Z}_{p}$-lattice $\Lambda_{j} \subset V\left(\lambda_{j}\right)$ given by $\Lambda_{j}=\mathfrak{U}_{H}^{-} \cdot v_{j}$ as before. Consider the $\mathcal{O}$-lattice $\mathcal{L}_{j}=\Lambda_{j} \otimes_{\mathbb{Z}_{p}} \mathcal{O}$ in $V\left(\lambda_{j}\right) \otimes_{\mathbb{Q}_{p}} L$; we then have a representation

$$
\rho_{j, o}: H_{\mathcal{O}} \rightarrow \operatorname{GL}\left(\mathcal{L}_{j}\right)
$$

over $\mathcal{O}$ such that $\rho_{j, o} \otimes_{\mathcal{O}} \tilde{\mathbb{Q}}_{p}=\rho_{j, L_{j}}^{\prime} \otimes_{L_{j}} \tilde{\mathbb{Q}}_{p}$. Every $\gamma$ in the inertial group $I_{j 1}=$ $\operatorname{Gal}\left(\tilde{\mathbb{Q}}_{p} / L_{j}\right) \subset \Xi$ preserves $\lambda_{j}$. Hence, $\rho_{j, o} \otimes_{\mathcal{O}} \tilde{\mathbb{Q}}_{p}$ and $\left(\rho_{j, o} \cdot \gamma\right) \otimes_{\mathcal{O}} \tilde{\mathbb{Q}}_{p}$ are equivalent representations and so there is $A_{\gamma} \in \operatorname{GL}\left(V\left(\lambda_{j}\right) \otimes_{\mathbb{Q}_{p}} \tilde{\mathbb{Q}}_{p}\right)$ with $\gamma(g) A_{\gamma}=A_{\gamma} g$ for all $g \in H\left(\tilde{\mathbb{Q}}_{p}\right)$. In fact, this identity makes sense and is still true for all $g \in \mathfrak{U}_{H}$. The matrix $A_{\gamma}$ takes $v_{j}$ to a multiple of $v_{j}$; we can normalize $A_{\gamma}$ to assume that $A_{\gamma} \cdot v_{j}=v_{j}$. Then $A_{\gamma}$ is uniquely determined. Since the action of $\Xi$ on $H$ is by diagram automorphisms, $\gamma$ preserves $\mathfrak{U}_{H}^{-}$. Hence

$$
A_{\gamma}\left(\Lambda_{j}\right)=A_{\gamma}\left(\mathfrak{U}_{H}^{-} \cdot v_{j}\right) \subset \gamma \cdot\left(\mathfrak{U}_{H}^{-}\right) \cdot A_{\gamma} v_{j} \subset \mathfrak{U}_{H}^{-} \cdot v_{j}=\Lambda_{j},
$$

i.e. $A_{\gamma}$ preserves $\mathcal{L}_{j}$ and hence $A_{\gamma}$ gives an equivalence of the $\mathcal{O}$-representations $\rho_{j, o}$ and $\rho_{j, o} \cdot \gamma$. We thus obtain $A: I_{j 1} \rightarrow \operatorname{GL}\left(\mathcal{L}_{j}\right), A(\gamma)=A_{\gamma}$, which we can see is a group homomorphism. Therefore we obtain a group scheme homomorphism

$$
\underline{\rho}_{j, o}:\left(\operatorname{Res}_{\mathcal{O}[w] / \mathcal{O}[v]}\left(H \otimes_{\mathcal{O}} \mathcal{O}[w]\right)\right)^{I_{j 1}} \rightarrow \mathrm{GL}\left(\left(\mathcal{L}_{j} \otimes_{\mathcal{O}} \mathcal{O}[w]\right)^{I_{j 1}}\right) .
$$

If $\lambda \in \mathbb{X}^{\bullet}\left(T_{H}\right)$ is a weight of $T_{H}$ and $\mathcal{L}_{j, \lambda}$ is the corresponding weight space of $\mathcal{L}_{j}$, so that $\mathcal{L}_{j}=\oplus_{\lambda} \mathcal{L}_{j, \lambda}$, then $A_{\gamma}\left(\mathcal{L}_{j, \lambda}\right)=\mathcal{L}_{j, \gamma \lambda}$. Since the $I_{j 1}$-cover $\mathcal{O}[w] / \mathcal{O}[v]$ is tame, the $\mathcal{O}[v]$-module $\left(\mathcal{L}_{j} \otimes_{\mathcal{O}} \mathcal{O}[w]\right)^{I_{j 1}}$ is finitely generated and projective and hence free (e.g. by [68]), of rank $d_{j}^{\prime}=\operatorname{dim}_{L_{j}}\left(V_{j}^{\prime}\right)$; Similarly, its direct summands 
$\left(\left(\oplus_{\gamma \lambda, \gamma \in I_{j 1}} \mathcal{L}_{j, \gamma \lambda}\right) \otimes_{\mathcal{O}} \mathcal{O}[w]\right)^{I_{j 1}}$ (the sum is for the weights in a $I_{j 1}$-orbit) are $\mathcal{O}[v]$ free. Choose a basis $\underline{b}$ that respects this decomposition; this allows us to identify the target $\mathrm{GL}\left(\left(\mathcal{L}_{j} \otimes_{\mathcal{O}} \mathcal{O}[w]\right)^{I_{j 1}}\right)$ with $\mathrm{GL}_{d_{j}^{\prime}}(\mathcal{O}[v])$. By restricting $\underline{\rho}_{j, o}$ to $\mathcal{O}\left[v^{ \pm 1}\right]$ we obtain a representation

$$
\underline{\rho}_{j, \mathcal{O}\left[v^{ \pm 1}\right]}^{\prime}:\left(\operatorname{Res}_{\mathcal{O}\left[w^{ \pm 1}\right] / \mathcal{O}\left[v^{ \pm 1}\right]}\left(H \otimes \mathcal{O} \mathcal{O}\left[w^{ \pm 1}\right]\right)\right)^{I_{j 1}} \rightarrow \operatorname{GL}_{d_{j}^{\prime}}\left(\mathcal{O}\left[v^{ \pm 1}\right]\right)
$$

that extends $\rho_{j, L_{j}}^{\prime}$. Set $d_{j}=d_{j}^{\prime} e_{j}$. By the definition of $\underline{G}$ (see 2.1.4), the source of (2.3.12) is isomorphic to $\underline{G} \otimes_{\mathcal{O}\left[u^{ \pm 1}\right]} \mathcal{O}\left[v^{ \pm 1}\right]$, and so we have a group scheme homomorphism

$$
\underline{G} \rightarrow \operatorname{Res}_{\mathcal{O}\left[v^{ \pm 1}\right] / \mathcal{O}\left[u^{ \pm 1}\right]}\left(\left(\operatorname{Res}_{\mathcal{O}\left[w^{ \pm 1}\right] / \mathcal{O}\left[v^{ \pm 1}\right]}\left(H \otimes_{\mathcal{O}} \mathcal{O}\left[w^{ \pm 1}\right]\right)\right)^{I_{j 1}}\right) .
$$

To obtain an extension

$$
\underline{\rho}_{j, \mathcal{O}\left[u^{ \pm 1}\right]}^{\prime}: \underline{G} \rightarrow \mathrm{GL}_{d_{j}}\left(\mathcal{O}\left[u^{ \pm 1}\right]\right)
$$

of $\rho_{j, L}^{\prime}$ we now compose (2.3.13) with $\operatorname{Res}_{\mathcal{O}\left[v^{ \pm 1}\right] / \mathcal{O}\left[u^{ \pm 1}\right]}\left(\underline{\rho}_{j, \mathcal{O}\left[v^{ \pm 1}\right]}^{\prime}\right)$ followed by the homomorphism $\operatorname{GL}_{d_{j}^{\prime}}\left(\mathcal{O}\left[v^{ \pm 1}\right]\right) \rightarrow \operatorname{GL}_{d_{j}}\left(\mathcal{O}\left[u^{ \pm 1}\right]\right)$ given by restriction of scalars from $\mathcal{O}\left[v^{ \pm 1}\right]$ to $\mathcal{O}\left[u^{ \pm 1}\right]$. Notice that

$$
\mathcal{O}[v] \simeq \mathcal{O}[u]^{e_{j}}
$$

as $\mathcal{O}[u]$-modules and so the target of the last map can be indeed identified with $\mathrm{GL}_{d_{j}}\left(\mathcal{O}\left[u^{ \pm 1}\right]\right)$. (Here and in other places, "extends" is meant in the sense that there is an equivalence between the base change of $\underline{\rho}_{j, \mathcal{O}\left[u^{ \pm 1}\right]}^{\prime}$ by $u \mapsto p$ and $\rho_{j, L}^{\prime}$.) We see that with the choice of basis of $V_{j, L}^{\prime}$ obtained by specializing $\underline{b}$ by $\mathcal{O}[u] \rightarrow L$, $u \mapsto p$, and using (2.3.14) above, the image $\rho_{j, L}^{\prime}(S)$ of $S$ is contained in the standard maximal torus of $\mathrm{GL}_{d_{j}}$. Then

$$
\iota_{j, L}: \mathcal{B}(G, L) \rightarrow \mathcal{B}\left(\mathrm{GL}\left(V_{j, L}^{\prime}\right), L\right)=\mathcal{B}\left(\mathrm{GL}_{d_{j}}, L\right),
$$

maps the apartment of the torus $S$ to the apartment of the standard maximal torus of $\mathrm{GL}_{d_{j}}$.

Step 2. We will now show that $\underline{\rho}_{j, \mathcal{O}\left[u^{ \pm 1}\right]}^{\prime}$ extends to a homomorphism

$$
\underline{\rho}_{j, \mathcal{O}[u]}^{\prime}: \underline{\mathcal{G}} \rightarrow \operatorname{Aut}_{\mathcal{O}[u]}\left(N_{\bullet}\right)
$$

of group schemes over $\operatorname{Spec}(\mathcal{O}[u])$. Here $N_{\bullet}=N_{j, \bullet} \subset \mathcal{O}\left[u^{ \pm 1}\right]^{d_{j}}$ is a periodic chain of finitely generated $\mathcal{O}[u]$-free rank $d_{j}$ submodules of $\mathcal{O}\left[u^{ \pm 1}\right]^{d_{j}}$ as in [59, 5.2]. Set $y:=$ $y_{j, L}=\iota_{j, L}(x)$; this choice will allow us to determine the chain $N_{\bullet}$. (For simplicity, in what follows, we sometimes omit the subscript $j$ ). This is done as follows: Recall that we have chosen a basis over $\mathcal{O}[u]$ that allows us to identify the apartments of the standard torus of $\mathrm{GL}_{d}$ over $L, L((u))$ and $k((u))$, and that $y$ is on the apartment of this torus over $L$. The identification gives a point $y_{L((u))}$ for $\mathrm{GL}_{d}(L((u)))$ which is in the apartment of this standard torus; this then corresponds to a $L \llbracket u \rrbracket$-lattice chain $\Lambda_{\bullet}^{\prime}$ in $L((u))^{d}$ and we take $N_{\bullet}=\Lambda_{\bullet}^{\prime} \cap \mathcal{O}\left[u^{ \pm 1}\right]^{d}$. We can see that $N_{\bullet}$ has the desired properties to form a periodic $\mathcal{O}[u]$-lattice chain. The construction of $\underline{G}$ also gives a point $x_{L((u))}$ in the building for $\underline{G}_{\otimes_{\mathcal{O}\left[u^{ \pm 1}\right]}} L((u))$. The point $x_{L((u))}$, by the same reason, then also maps to the point $y_{L((u))}$ for $\mathrm{GL}_{d}(L((u)))$. (The point $y_{L((u))}$ is also the image of $x_{L((u))}$ by a map $\iota_{L((u))}: \mathcal{B}(\underline{G}, L((u))) \rightarrow \mathcal{B}\left(\mathrm{GL}_{d}, L((u))\right)$ that can be defined as before using our choices.) Since for $\underline{\mathcal{G}}=\operatorname{Spec}(\mathcal{A})$ we have $\mathcal{A}=\mathcal{A}\left[u^{-1}\right] \cap\left(\mathcal{A} \otimes_{\mathcal{O}[u]} L \llbracket u \rrbracket\right)$, it will be enough to show that ${\underline{\rho_{j, \mathcal{O}}^{\prime}\left[u^{ \pm 1}\right]}} \otimes_{\mathcal{O}\left[u^{ \pm 1}\right]} L((u))$ extends to a group scheme homomorphism of the corresponding parahoric group 
schemes over $L \llbracket u \rrbracket$; this now follows from our choice of $N_{\bullet}$ above. We can now see that

$$
\underline{\rho}_{\mathcal{O}[u]}:=\prod_{j} \prod_{\tau} \underline{\rho}_{j, \mathcal{O}[u]}^{\prime}: \underline{\mathcal{G}} \rightarrow \mathcal{H}:=\prod_{j} \prod_{\tau} \operatorname{Aut}_{\mathcal{O}[u]}\left(N_{j, \bullet}\right)
$$

extends the base change $\rho_{L}=\rho \otimes_{\mathbb{Q}_{p}} L: G_{L} \rightarrow \prod_{j} \prod_{\tau} \mathrm{GL}\left(V_{j, L}^{\prime}\right) \subset \mathrm{GL}\left(V_{L}\right)$.

Step 3. It remains to show that $\underline{\rho}_{\mathcal{O}[u]}$ satisfies condition $\left({ }^{*}\right)$ above.

This will be obtained using the results and arguments of the previous paragraphs by observing that $\underline{\rho}_{\mathcal{O}[u]} \otimes_{\mathcal{O}[u]} k((u))$ is minuscule. In fact, by construction, this representation satisfies the assumptions described in 1.2.27.

Set $F=k((u)), \tilde{F}=k((w))$. As in 1.2 .27 we see that $\underline{\rho}_{\mathcal{O}[u]} \otimes_{\mathcal{O}[u]} \tilde{F}$ produces a $\underline{G}(\tilde{F})=H(\tilde{F})$-equivariant and $\operatorname{Gal}(\tilde{F} / F)$-equivariant toral embedding

$$
\iota_{\tilde{F}}: \mathcal{B}(H, \tilde{F}) \rightarrow \mathcal{B}\left(\mathrm{GL}_{n}, \tilde{F}\right),
$$

with $n=\operatorname{dim}_{L}\left(V_{L}\right)$. This embedding is obtained using the decomposition into irreducibles and the descent data given as above. By its construction, $\iota_{\tilde{F}}$ has the following property: It maps the apartment of the standard torus of $H(\tilde{F})$ to the standard apartment of $\mathrm{GL}_{n}(\tilde{F})$ compatibly with the identifications of apartments over $\tilde{F}$ and $\tilde{\mathbb{Q}}_{p}$ and with the maps between the buildings over $\tilde{\mathbb{Q}}_{p}$ as above. It also sends $x_{F}$ to $y_{F}$ (where these are points are determined from $x$ and $y$ by our choices above as in 2.1.4). We now see that 1.3.13, which is a version of Proposition 1.3.3 in the equicharacteristic case, implies the desired statement.

2.3.15. We now return to the previous set-up, as in 2.3.1 As in 1.1.11, $\mathcal{G S P} z$ is the stabilizer of a periodic self-dual (with respect to the form $\psi$ ) lattice chain $\mathcal{L}=$ $\left\{\Lambda^{i}\right\}_{i \in \mathbb{Z}}$ in $V$. Index the chain as in 1.1.11, in particular, assume that $\left(\Lambda^{i}\right)^{\vee}=\Lambda^{-i-a}$ with $a=0$ or 1 . Set $V^{\prime}=\oplus_{i=-(r-1)-a}^{r-1} V$ equipped with the perfect alternating $K$ bilinear form $\psi^{\prime}$ as in 1.1.11. Consider the lattice $V_{\mathbb{Z}_{p}}^{\prime}=\oplus_{i=-(r-1)-a}^{r-1} p \Lambda^{i} \subset V^{\prime}$; then $V_{\mathbb{Z}_{p}}^{\prime} \subset V_{\mathbb{Z}_{p}}^{\prime \vee}$. The closed immersion $\mathcal{H}_{z} \hookrightarrow \operatorname{GSp}\left(V_{\mathbb{Z}_{p}}^{\prime}, \psi^{\prime}\right)$ composed with $\rho: \mathcal{G}_{x} \hookrightarrow \mathcal{H}_{z}$ gives a closed group scheme immersion

$$
\rho^{\prime}: \mathcal{G}_{x} \hookrightarrow \operatorname{GSp}\left(V_{\mathbb{Z}_{p}}^{\prime}, \psi^{\prime}\right) \subset \mathrm{GL}\left(V_{\mathbb{Z}_{p}}^{\prime}\right) .
$$

This shows that by composing $\rho$ with the embedding above, we can assume that the point $y$ is hyperspecial. The corresponding local model $\mathrm{M}_{\mathrm{GL}\left(V^{\prime}\right),\left\{\mu_{0}^{\prime}\right\}, y}^{\mathrm{loc}}$ over $\mathbb{Z}_{p}$ is the smooth Grassmannian $\operatorname{Gr}\left(V_{\mathbb{Z}_{p}}^{\prime}\right)$ classifying subbundles $\mathcal{F} \subset V_{\mathbb{Z}_{p}}^{\prime} \otimes_{\mathbb{Z}_{p}} \mathcal{O}_{S}$ of rank $\operatorname{dim}_{\mathbb{Q}_{p}}\left(V^{\prime}\right)$. We thus obtain

Corollary 2.3.16. Assume $\rho: G \rightarrow \operatorname{GSp}(V, \psi)$ comes from a Hodge embedding as above. We can find a new Hodge embedding $\rho^{\prime}: G \rightarrow \operatorname{GSp}\left(V^{\prime}, \psi^{\prime}\right)$ and a lattice $V_{\mathbb{Z}_{p}}^{\prime} \subset V^{\prime}$ with $V_{\mathbb{Z}_{p}}^{\prime} \subset V_{\mathbb{Z}_{p}}^{\prime \vee}$, such that $\rho^{\prime}$ induces a closed immersion

$$
\mathrm{M}_{G,\{\mu\}, x}^{\text {loc }} \hookrightarrow \operatorname{Gr}\left(V_{\mathbb{Z}_{p}}^{\prime}\right) \otimes_{\mathbb{Z}_{p}} \mathcal{O}_{E}
$$

of schemes over $\mathcal{O}_{E}$.

\section{Deformations of $p$-Divisible Groups}

\subsection{A construction for the universal deformation.}

3.1.1. We continue to use the notations introduced in (1.1.1). In particular, we write $W=W(k)$ and $K_{0}=W[1 / p]$. Unless we mention otherwise, we assume $p>2$. 
The aim of this section is to construct the versal deformation space of a $p$-divisible group over $k$ using Zink's theory of displays.

3.1.2. Let $R$ be a complete local ring with residue field $k$, and maximal ideal $\mathfrak{m}$.

Recall [74] $\S 2$ (see also [50]), that we have a subring $\widehat{W}(R)=W(k) \oplus \mathbb{W}(\mathfrak{m}) \subset$ $W(R)$, where $\mathbb{W}(\mathfrak{m}) \subset W(R)$ consists of Witt vectors $\left(w_{i}\right)_{i \geq 1}$ such that $w_{i} \in \mathfrak{m}$ and $\left\{w_{i}\right\}_{i \geq 1}$ goes to $0 \mathfrak{m}$-adically. We write $\varphi$ for the Frobenius on $\widehat{W}(R)$ and $V$ for the Verschiebung.

Let $I_{R} \subset \widehat{W}(R)$ denote the kernel of the projection $\widehat{W}(R) \rightarrow R$. We recall that the Verschiebung $V$ on $\widehat{W}(R)$ maps $\widehat{W}(R)$ isomorphically to $I_{R}$, and we write $V^{-1}: I_{R} \rightarrow \widehat{W}(R)$ for the inverse map. Note that

$$
\varphi\left(I_{R}\right)=\varphi(V(\widehat{W}(R))=(\varphi V)(\widehat{W}(R))=p \widehat{W}(R) .
$$

3.1.3. Recall [73] that a Dieudonné display over $R$ is a tuple $\left(M, M_{1}, \Phi, \Phi_{1}\right)$ where

(i) $M$ is a finite free $\widehat{W}(R)$-module.

(ii) $M_{1} \subset M$ is an $\widehat{W}(R)$-submodule such that

$$
I_{R} M \subset M_{1} \subset M
$$

and $M / M_{1}$ is a projective $R$-module.

(iii) $\Phi: M \rightarrow M$ is a $\varphi$-semi-linear map

(iv) $\Phi_{1}: M_{1} \rightarrow M$ is a $\varphi$-semi-linear map whose image generates $M$ as a $\widehat{W}(R)$ module, and which satisfies

$$
\Phi_{1}(V(w) m)=w \Phi(m) ; \quad w \in \widehat{W}(R), m \in M .
$$

We will sometimes write $\bar{M}=M / I_{R} M$ and $\bar{M}_{1}=M_{1} / I_{R} M$. We think of $\bar{M}$ as a filtered $R$-module, with $\operatorname{Fil}^{0} \bar{M}=\bar{M}$, and $\operatorname{Fil}^{1} \bar{M}=\bar{M}_{1}$.

If we take $w=1$ and $m \in M_{1}$, in the equation in (iv) above, we obtain

$$
\Phi(m)=\varphi V(1) \Phi_{1}(m)=p \Phi_{1}(m) .
$$

We will be particularly interested in cases where $W(R)$, and hence $\widehat{W}(R)$, is $p$-torsion free. This condition holds when $R$ is $p$-torsion free, or when $p \cdot R=0$, and $R$ is reduced. In this case, the tuple $\left(M, M_{1}, \Phi, \Phi_{1}\right)$ is determined by $\left(M, M_{1}, \Phi_{1}\right)$ satisfying (i), (ii) and (iv) above. Indeed, we define $\Phi$ by setting $\Phi(m)=\Phi_{1}(V(1) m)$ for $m \in M$. Then for $w \in \widehat{W}(R)$ and $m \in M$ we have

$$
\left.p \Phi_{1}(V(w) m)=\Phi_{1}(V(w) V(1) m)=p w \Phi_{1}(V(1) m)\right)=p w \Phi(m),
$$

and hence $\Phi_{1}(V(w) m)=w \Phi(m)$ as $\widehat{W}(R)$ is $p$-torsion free.

When $W(R)$ is $p$-torsion free, we will also refer to the tuple $\left(M, M_{1}, \Phi_{1}\right)$ satisfying (i), (ii) and (iv) as a Dieudonné display over $R$.

3.1.4. Let $\left(M, M_{1}, \Phi, \Phi_{1}\right)$ be a Dieudonné display over $R$. The condition (ii) implies that we may write $M$ as a sum of $\widehat{W}(R)$-submodules $M=L \oplus T$ such that $M_{1}=$ $L \oplus I_{R} T$. Such a direct sum is called a normal decomposition for $M$.

Denote by $\widetilde{M}_{1}$ the image of the $\widehat{W}(R)$-module homomorphism

$$
\varphi^{*}(i): \varphi^{*} M_{1}:=\widehat{W}(R) \otimes_{\varphi, \widehat{W}(R)} M_{1} \rightarrow \varphi^{*} M=\widehat{W}(R) \otimes_{\varphi, \widehat{W}(R)} M
$$

induced by the inclusion $i: M_{1} \rightarrow M$.

Note that $\widetilde{M}_{1}$ and the notion of a normal decomposition depends only on $M$ and the submodule $M_{1}$ and not on $\Phi$ and $\Phi_{1}$. 
Lemma 3.1.5. Suppose that $W(R)$ is p-torsion free. Let $M$ be a free $\widehat{W}(R)$-module, and $M_{1} \subset M$ a submodule, with $I_{R} M \subset M_{1}$ and $M / M_{1}$ a projective $R$-module, and let $M=L \oplus T$ be a normal decomposition for $M$. Then

a) The $\widehat{W}(R)$-module $\widetilde{M}_{1}$ is isomorphic to $\varphi^{*}(L) \oplus p \varphi^{*}(T) \simeq \widehat{W}(R)^{d}$, with $d=$ $\mathrm{rk}_{\widehat{W}(R)} M$, and in particular depends only on the reduction of $\left(M, M_{1}\right)$ modulo $p$.

b) If $\left(M, M_{1}, \Phi_{1}\right)$ is a Dieudonné display over $R$, then the linearization of $\Phi_{1}$, $\Phi_{1}^{\#}: \varphi^{*} M_{1} \rightarrow M$ factors as a composition

$$
\Phi_{1}^{\#}: \varphi^{*} M_{1} \rightarrow \widetilde{M}_{1} \stackrel{\Psi}{\rightarrow} M
$$

with $\Psi$ an $\widehat{W}(R)$-module isomorphism.

c) Conversely, suppose we are given

$$
\Psi: \widetilde{M}_{1}:=\operatorname{Im}\left(\varphi^{*} M_{1} \rightarrow \varphi^{*} M\right) \stackrel{\sim}{\longrightarrow} M .
$$

There there is a unique Dieudonné display over $R,\left(M, M_{1}, \Phi_{1}\right)$ which produces our given $\left(M, M_{1}, \Psi\right)$ via the construction in (b).

Proof. (a) follows immediately from that fact that $\varphi\left(I_{R}\right)=p \widehat{W}(R)$.

For (b) we first show that $\Phi_{1}^{\#}: \varphi^{*} M_{1} \rightarrow M$ factors through $\widetilde{M}_{1}$. It is enough to show that $p \Phi_{1}^{\#}$ vanishes on the kernel $K$ of $\varphi^{*}(i): \varphi^{*} M_{1} \rightarrow \varphi^{*} M$. But $p \Phi_{1}=\Phi_{\mid M_{1}}$ and so $p \Phi_{1}^{\#}=\Phi^{\#} \circ \varphi^{*}(i)$; this obviously vanishes on $K$. We write $\Phi_{1}^{\#}=\Psi \circ$ $\left(\varphi^{*} M_{1} \rightarrow \widetilde{M}_{1}\right)$ with a surjective $\Psi: \widetilde{M}_{1} \simeq M$ which is necessarily an isomorphism, as $\widetilde{M}_{1}$ and $M$ are free over $\widehat{W}(R)$ of the same rank.

For (c) define $\Phi_{1}: M_{1} \rightarrow M$ by

$$
\Phi_{1}\left(m_{1}\right)=\Psi\left(1 \otimes m_{1}\right)
$$

where $1 \otimes m_{1}$ denotes the image of $1 \otimes m_{1} \in \widehat{W}(R) \otimes_{\varphi, \widehat{W}(R)} M_{1}=\varphi^{*} M_{1}$ in $\varphi^{*} M$. Them $\Phi_{1}$ is clearly $\varphi$-linear and its linearization $\Phi_{1}^{\#}: \varphi^{*} M_{1} \rightarrow M$ is surjective.

3.1.6. Let $R \rightarrow R^{\prime}$ be a morphism of complete local rings with residue field $k$. A Dieudonné display $\left(M, M_{1}, \Phi, \Phi_{1}\right)$ over $R$, has a base change to $R^{\prime}$ (cf. [75] Defn. 20), given by $M_{R^{\prime}}=M \otimes_{\widehat{W}(R)} \widehat{W}\left(R^{\prime}\right)$, and

$$
M_{R^{\prime}, 1}=\operatorname{ker}\left(M_{R^{\prime}} \rightarrow M / M_{1} \otimes_{R} R^{\prime}\right)=\operatorname{Im}\left(M_{1} \otimes_{\widehat{W}(R)} \widehat{W}\left(R^{\prime}\right) \rightarrow M_{R^{\prime}}\right)+I_{R^{\prime}} M_{R^{\prime}} .
$$

Then $\Phi$ on $M_{R^{\prime}}$ is defined as the $\varphi$ semi-linear extension of $\Phi$ on $M$. The map $\Phi_{1}$ on $M_{R^{\prime}, 1}$ is the unique $\varphi$-semilinear map $M_{R^{\prime}, 1} \rightarrow M_{R^{\prime}}$ which satisfies

$$
\Phi_{1}\left(w \otimes m_{1}\right)=\varphi(w) \otimes \Phi_{1}\left(m_{1}\right) \quad w \in \widehat{W}\left(R^{\prime}\right), m_{1} \in M_{1}
$$

and

$$
\Phi_{1}(V(w) \otimes m)=w \otimes \Phi(m) \quad w \in \widehat{W}\left(R^{\prime}\right) . m \in M .
$$

The existence and uniqueness of such a map follows, as in loc. cit., from the existence of a normal decomposition. In particular, if $R \rightarrow R^{\prime}$ is surjective, we have the notion of a deformation to $R$ of a display over $R^{\prime}$.

If $W(R)$ and $W\left(R^{\prime}\right)$ are $p$-torsion free, then using a normal decomposition one finds that there is a natural isomorphism $\widetilde{M}_{R^{\prime}, 1} \cong \widetilde{M}_{1} \otimes_{\widehat{W}(R)} \widehat{W}\left(R^{\prime}\right)$, and the 
diagram

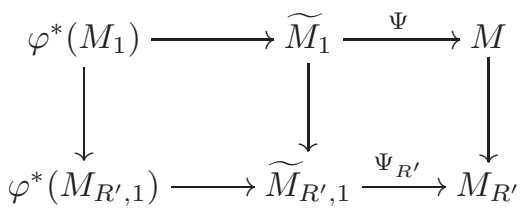

commutes. Here $\Psi_{R^{\prime}}$ denotes the map associated to the Dieudonné display over $R^{\prime}$ by Lemma 3.1 .5

3.1.7. Let $\mathscr{G}$ be a $p$-divisible group over $R$, and denote by $\mathbb{D}(\mathscr{G})$ its contravariant Dieudonné crystal. By the main theorem of $[73, \mathbb{D}(\mathscr{G})(\widehat{W}(R))$ has a natural structure of Dieudonné display over $R$, and the functor $\mathscr{G} \mapsto \mathbb{D}(\mathscr{G})(\widehat{W}(R))$ induces an anti-equivalence between $p$-divisible groups over $R$, and Dieudonné $\widehat{W}(R)$-display over $R$. More precisely, the equivalence of loc. cit. uses the covariant Dieudonné crystal, and we compose the functor defined there with Cartier duality. Under this anti-equivalence, base change for Dieudonné displays, defined in the previous paragraph corresponds to base change for $p$-divisible groups [50] Thm. 3.19.

3.1.8. Let $\mathscr{G}_{0}$ be a $p$-divisible group over $k$. We now use the above to construct the versal deformation space of $\mathscr{G}_{0}$.

Let $\mathbb{D}=\mathbb{D}\left(\mathscr{G}_{0}\right)(W)$, and let $\left(\mathbb{D}, \mathbb{D}_{1}, \Phi, \Phi_{1}\right)$ be the Dieudonné display corresponding to $\mathscr{G}_{0}$. By Lemma 3.1.5, this data is given by an isomorphism $\Psi_{0}: \widetilde{\mathbb{D}}_{1}=$ $\varphi^{*}\left(\mathbb{D}_{1}\right) \cong \mathbb{D}$.

The filtration on $\mathbb{D}\left(\mathscr{G}_{0}\right)(k)$ corresponds to a parabolic subgroup $P_{0} \subset \mathrm{GL}\left(\mathbb{D} \otimes_{W}\right.$ $k$ ). Fix a lifting of $P_{0}$ to a parabolic subgroup $P \subset \mathrm{GL}(\mathbb{D})$. Write $\mathrm{M}^{\text {loc }}=\mathrm{GL}(\mathbb{D}) / P$ and denote by $\widehat{\mathrm{M}}^{\mathrm{loc}}=\operatorname{Spf} R$, the completion of $\mathrm{GL}(\mathbb{D}) / P$ along the image of the identity in $\mathrm{GL}\left(\mathbb{D} \otimes_{W} k\right)$, so that $R$ is a power series ring over $W$.

Set $M=\mathbb{D} \otimes_{W} \widehat{W}(R)$, and let $\bar{M}_{1} \subset M / I_{R} M$ be the direct summand corresponding to the parabolic subgroup $g \mathrm{Pg}^{-1} \subset \mathrm{GL}(\mathbb{D})$ over $\widehat{\mathrm{M}}^{\mathrm{loc}}$, where $g \in(\mathrm{GL}(\mathbb{D}) / P)(R)$ is the universal point. We denote by $M_{1} \subset M$ the preimage of $\bar{M}_{1}$ in $M$. Let $\Psi: \widetilde{M}_{1} \cong M$ be an $\widehat{W}(R)$-linear isomorphism which reduces to $\Psi_{0} \bmod \mathfrak{m}_{R}$. Then $\left(M, M_{1}, \Psi\right)$ corresponds to a Dieudonné display over $R$, and hence to a $p$-divisible group $\mathscr{G}_{R}$ over $R$ which deforms $\mathscr{G}_{0}$.

Lemma 3.1.9. Let $\mathfrak{a}_{R}=\mathfrak{m}_{R}^{2}+p R$. There is a canonical commutative diagram

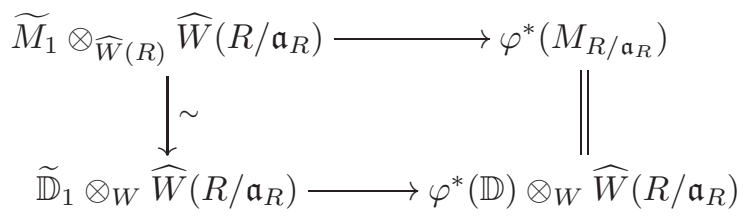

where the horizontal maps are induced by the natural inclusions $\widetilde{M}_{1} \rightarrow \varphi^{*}\left(M_{R}\right)$ and $\widetilde{\mathbb{D}}_{1} \rightarrow \varphi^{*}(\mathbb{D})$.

Proof. $L \oplus T$ be a normal decomposition for $\left(M_{R / \mathfrak{a}_{R}}, M_{R / \mathfrak{a}_{R}, 1}\right)$, and let $L_{0} \oplus T_{0}$ be the induced normal decomposition for $\left(\mathbb{D}, \mathbb{D}_{1}\right)$. Observe that the Frobenius on $\widehat{W}\left(R / \mathfrak{a}_{R}\right)$ factors as

$$
\widehat{W}\left(R / \mathfrak{a}_{R}\right) \rightarrow W \stackrel{\varphi}{\rightarrow} W \rightarrow W\left(R / \mathfrak{a}_{R}\right)
$$


Hence the submodule $\varphi^{*}(T) \subset \varphi^{*}\left(M_{R / \mathfrak{a}_{R}}\right)$ is identified with $\varphi^{*}\left(T_{0}\right) \otimes_{W} \widehat{W}\left(R / \mathfrak{a}_{R}\right) \subset$ $\mathbb{D} \otimes_{W} \widehat{W}\left(R / \mathfrak{a}_{R}\right)$. An analogous remark applies to $L$.

For any $\mathbb{Z}_{p}$-module $N$ write $p \otimes N=p \mathbb{Z}_{p} \otimes_{\mathbb{Z}_{p}} N$. Then

$$
\begin{aligned}
\widetilde{M}_{1} \otimes_{\widehat{W}(R)} & \widehat{W}\left(R / \mathfrak{a}_{R}\right) \cong \varphi^{*}(L) \oplus p \otimes \varphi^{*}(T) \\
& \cong\left(\varphi^{*}\left(L_{0}\right) \oplus p \otimes \varphi^{*}\left(T_{0}\right)\right) \otimes_{W} \widehat{W}\left(R / \mathfrak{a}_{R}\right)=\widetilde{\mathbb{D}}_{1} \otimes_{W} \widehat{W}\left(R / \mathfrak{a}_{R}\right) .
\end{aligned}
$$

This produces the left isomorphism in the lemma, and one checks immediately the diagram commutes, and is independent of the choice of normal decomposition.

3.1.11. We say that $\Psi$ is constant modulo $\mathfrak{a}_{R}$ if the composite map

$$
\widetilde{\mathbb{D}}_{1} \otimes_{W} \widehat{W}\left(R / \mathfrak{a}_{R}\right) \cong \widetilde{M}_{1} \otimes_{\widehat{W}(R)} \widehat{W}\left(R / \mathfrak{a}_{R}\right) \stackrel{\Psi}{\sim} M_{R / \mathfrak{a}_{R}} \cong \mathbb{D} \otimes_{W} \widehat{W}\left(R / \mathfrak{a}_{R}\right)
$$

is equal to $\Psi_{0} \otimes 1$.

Lemma 3.1.12. If $\Psi$ is constant mod $\mathfrak{a}_{R}$ then the deformation $\mathscr{G}_{R}$ of $\mathscr{G}_{0}$ is versal.

Proof. We have two displays over $R / \mathfrak{a}_{R}$. One obtained from $\left(M, M_{1}, \Phi, \Phi_{1}\right)$ by the base change $R \rightarrow R / \mathfrak{a}_{R}$, and one obtained from $\left(\mathbb{D}, \mathbb{D}_{1}, \Phi, \Phi_{1}\right)$ by the base change $k \rightarrow R / \mathfrak{a}_{R}$. We denote the corresponding morphisms $\Phi_{1}$ by $\Phi_{1}$ and $\Phi_{1,0}$ respectively.

Let $\hat{M}_{R / \mathfrak{a}_{R}, 1} \subset M_{R / \mathfrak{a}_{R}}$ be the submodule

$$
\begin{aligned}
\hat{M}_{R / \mathfrak{a}_{R}, 1}=M_{R / \mathfrak{a}_{R}, 1} & +\mathbb{W}\left(\mathfrak{m}_{R} / \mathfrak{a}_{R}\right) M_{R / \mathfrak{a}_{R}} \\
& =\mathbb{D}_{1} \otimes_{W} \widehat{W}\left(R / \mathfrak{a}_{R}\right)+\mathbb{W}\left(\mathfrak{m}_{R} / \mathfrak{a}_{R}\right) M_{R / \mathfrak{a}_{R}} \subset M_{R / \mathfrak{a}_{R}} .
\end{aligned}
$$

We regard $R / \mathfrak{a}_{R} \rightarrow k$ as a thickening with trivial divided powers. By 73 Thm. 3, the morphisms $\Phi_{1}$ and $\Phi_{1,0}$ extend uniquely to $\varphi$-semilinear maps

$$
\hat{\Phi}_{1}, \hat{\Phi}_{1,0}: \hat{M}_{R / \mathfrak{a}_{R}, 1} \rightarrow M_{R / \mathfrak{a}_{R}} .
$$

We claim that if $\Psi$ is constant $\bmod \mathfrak{a}_{R}$ then $\hat{\Phi}_{1}=\hat{\Phi}_{1,0}$. Assuming this, the lemma follows from 73 Thm 4 , and the versality of the filtration $\bar{M}_{1} \subset M / \mathfrak{a}_{R}=\mathbb{D} \otimes_{W} R$. (As well as, of course, the main theorem of loc. cit. giving the equivalence between displays and $p$-divisible groups.)

To show the claim, note that we may regard $\mathfrak{m}_{R} / \mathfrak{a}_{R}$ as a $\widehat{W}\left(R / \mathfrak{a}_{R}\right)$-submodule of $\mathbb{W}\left(\mathfrak{m}_{R} / \mathfrak{a}_{R}\right)$, by sending $a \in \mathfrak{m}_{R} / \mathfrak{a}_{R}$ to $[a]$. Let $L \oplus T$ be a normal decomposition for $\left(M_{R / \mathfrak{a}_{R}}, M_{R / \mathfrak{a}_{R}, 1}\right)$. Then $\hat{M}_{R / \mathfrak{a}_{R}, 1}=\mathfrak{a}_{R} T \oplus L \oplus I_{R / \mathfrak{a}_{R}} T$, and $\hat{\Phi}_{1}$ is given by sending $\mathfrak{a}_{R} T$ to 0 , and on $L \oplus I_{R / \mathfrak{a}_{R}} T$, is given by the map

$$
L \oplus I_{R / \mathfrak{a}_{R}} T \rightarrow \varphi^{*}(L) \oplus p \otimes \varphi^{*}(T)=\widetilde{M}_{1} \otimes_{\widehat{W}(R)} \widehat{W}\left(R / \mathfrak{a}_{R}\right) \stackrel{\Psi}{\rightarrow} M_{R / \mathfrak{a}_{R}} .
$$

In particular, we see that there is a natural map $\varphi^{*}\left(\hat{M}_{R / \mathfrak{a}_{R}, 1}\right) \rightarrow \widetilde{M}_{1} \otimes_{\widehat{W}(R)}$ $\widehat{W}\left(R / \mathfrak{a}_{R}\right)$, which is independent of the choice of $\Psi$, and that the linearization $\hat{\Phi}_{1}^{\#}$ of $\hat{\Phi}_{1}$ factors through this map and is induced by $\Psi$. As in the proof of Lemma 3.1.9, this map depends only on $T_{0}$ and not on $T$. An analogous remark applies to $\hat{\Phi}_{1,0}$. Thus, we obtain a diagram

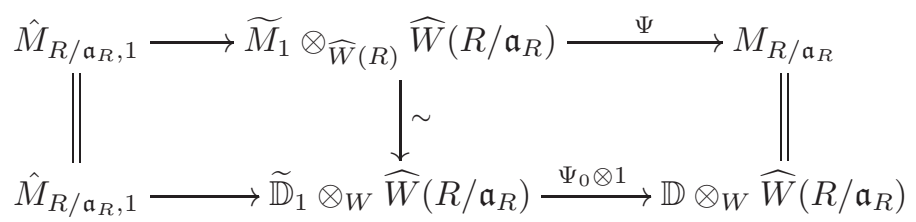


where the composite horizontal maps are $\hat{\Phi}_{1}$ and $\hat{\Phi}_{1,0}$ respectively, the left square commutes, and the right square commutes if $\Psi$ is constant mod $\mathfrak{a}_{R}$. This proves the claim.

3.1.14. We assume from now on that $\Psi$ is constant $\bmod \mathfrak{a}_{R}$, so that $\mathscr{G}_{R}$ is versal. Equivalently, $M=M_{R}$ is versal for deformations of displays. Let $S^{\prime} \rightarrow S$ be a surjection of $W$-algebras. If $\mathscr{G}_{S}$ is a $p$-divisible group over $S$, we denote by $\operatorname{Def}\left(\mathscr{G}_{S} ; S^{\prime}\right)$ the set of isomorphism classes of deformations of $\mathscr{G}_{S}$ to $S^{\prime}$. We will apply this when $S^{\prime} \rightarrow S$ has nilpotent kernel, in which case a deformation of $\mathscr{G}_{S}$ to $S^{\prime}$ has no automorphisms. If $f: A \rightarrow S$ is a map of $W$-algebras, we denote by $\operatorname{Def}\left(f ; S^{\prime}\right)$ the set of lifts of $f$ to a map $A \rightarrow S^{\prime}$. For any ring $A$ we denote by $A[\epsilon]=A[X] / X^{2}$ the dual numbers over $A$.

Lemma 3.1.15. Let $K / K_{0}$ be a finite extension, with ring of integers $\mathcal{O}_{K}$. Let $\xi: R \rightarrow \mathcal{O}_{K}$ be a map of $W$-algebras, and $\mathscr{G}_{\xi}$ the induced p-divisible group over $\mathcal{O}_{K}$. The map

$$
\operatorname{Def}\left(\xi ; \mathcal{O}_{K}[\epsilon]\right) \rightarrow \operatorname{Def}\left(\mathscr{G}_{\xi} ; \mathcal{O}_{K}[\epsilon]\right)
$$

is a bijection.

Proof. Let $R_{\mathcal{O}_{K}}=R \otimes_{W} \mathcal{O}_{K}$. Let $\xi_{K}: R_{\mathcal{O}_{K}} \rightarrow \mathcal{O}_{K}$ be the induced map of $\mathcal{O}_{K^{-}}$ algebras, and $I=\operatorname{ker}\left(\xi_{K}\right) \subset R_{\mathcal{O}_{K}}$. Then $\operatorname{Def}\left(\xi ; \mathcal{O}_{K}[\epsilon]\right)$ is in bijection with the set of lifts of $\xi_{K}$ to a map of $\mathcal{O}_{K}$-algebras $R_{\mathcal{O}_{K}} \rightarrow \mathcal{O}_{K}[\epsilon]$, and the latter set is naturally in bijection with $\operatorname{Hom}_{\mathcal{O}_{K}}\left(I / I^{2}, \epsilon \cdot \mathcal{O}_{K}\right)$. In particular, $\operatorname{Def}\left(\xi ; \mathcal{O}_{K}[\epsilon]\right)$ is naturally a free $\mathcal{O}_{K}$-module. Similarly $\operatorname{Def}\left(\mathscr{G}_{\xi} ; \mathcal{O}_{K}[\epsilon]\right)$ is naturally a free $\mathcal{O}_{K}$-module; it may be identified with the tangent space to a point in a Grassmannian over $\mathcal{O}_{K}$. One checks easily that the map in the lemma is a map of $\mathcal{O}_{K}$-modules.

Now let $\xi_{0}: R \rightarrow k^{\prime}$ be the map to the residue field $k^{\prime}$ over $\mathcal{O}_{K}$. We again denote by $\mathscr{G}_{0}$ the base change of $\mathscr{G}_{0}$ to $k^{\prime}$. Consider the diagram

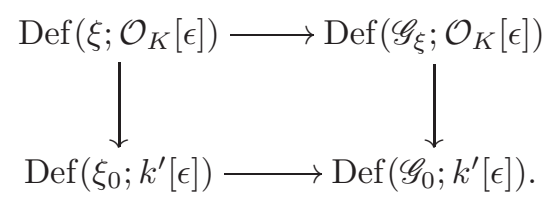

Here the vertical maps are given by specializing maps, respectively $p$-divisible groups via the map $\mathcal{O}_{K} \rightarrow k^{\prime}$. The map at the bottom is obtained from the map of $\mathcal{O}_{K}$-modules in the top row by applying $\otimes_{\mathcal{O}_{K}} k$. This is obvious for the term on the left, and for the term on the right it follows from the description of $\operatorname{Def}\left(\mathscr{G}_{\xi}, \mathcal{O}_{K}[\epsilon]\right)$ and $\operatorname{Def}\left(\mathscr{G}_{0}, k^{\prime}[\epsilon]\right)$ in terms of Grothendieck-Messing theory. Since $\mathscr{G}_{R}$ is versal the map on the bottom is an isomorphism, and hence so is the map of free $\mathcal{O}_{K}$-modules at the top.

3.1.16. We end with the following lemma, which will be needed later

Lemma 3.1.17. Let $A$ be a complete local, p-torsion free, $W$-algebra, with $\mathfrak{m}_{A}^{N} \subset$ $p A$ for some integer $N$. Let $M_{A}=\left(M_{A}, M_{A, 1}, \Phi, \Phi_{1}\right)$ be a deformation of the Dieudonné display $\mathbb{D}$ to a Dieudonné display over $A$. Then there is a unique, Frobenius equivariant map

$$
\mathbb{D} \otimes_{\mathbb{Z}_{p}} \mathbb{Q}_{p} \rightarrow M_{A} \otimes_{\mathbb{Z}_{p}} \mathbb{Q}_{p}
$$

lifting the identity on $\mathbb{D}$. 
Proof. Lift the identity on $\mathbb{D}$, to an arbitrary $W$-linear map $s: \mathbb{D} \rightarrow M_{A}$. Denote by $\Phi_{0}$ the Frobenius on $\mathbb{D}$. Then

$$
\Phi \circ s \circ \Phi_{0}^{-1}-s \in \mathbb{W}\left(\mathfrak{m}_{A}\right) \operatorname{Hom}_{W}\left(\mathbb{D}, M_{A}\right) \otimes_{\mathbb{Z}_{p}} \mathbb{Q}_{p} .
$$

Hence, it suffices to give a complete separated topology $\tau$ on $\widehat{W}(A) \otimes_{\mathbb{Z}_{p}} \mathbb{Q}_{p}$ such that for any $x \in \mathbb{W}\left(\mathfrak{m}_{A}\right), p^{-m} \varphi^{m}(x) \rightarrow 0$. Indeed, then the sum

$\tilde{s}=s+\sum_{m=0}^{\infty} \Phi^{m} \circ\left(\Phi \circ s \circ \Phi_{0}^{-1}-s\right) \circ \Phi_{0}^{-m}=s+\sum_{m=0}^{\infty} \Phi^{m+1} \circ s \circ \Phi_{0}^{-m-1}-\Phi^{m} \circ s \circ \Phi_{0}^{-m}$

converges to an element of $\operatorname{Hom}_{W}(\mathbb{D}, M) \otimes_{\mathbb{Z}_{p}} \mathbb{Q}_{p}$ in $\tau$ and is Frobenius invariant.

To define $\tau$ we consider the maps defined by the Witt polynomials

$$
\widehat{W}(A) \otimes_{\mathbb{Z}_{p}} \mathbb{Q}_{p} \stackrel{\prod_{n} \mathbf{w}_{n}}{\longrightarrow} \prod_{n \geq 0} A[1 / p] .
$$

Now $A$ is equipped with its $p$-adic topology. This induces a topology on $A[1 / p]$, and hence on $\prod_{n>0} A[1 / p]$. We take $\tau$ to be the coarsest topology such that the above map is continuous.

To see that $\tau$ has the required property, let $x=\left(x_{0}, x_{1}, \ldots\right) \in \mathbb{W}\left(\mathfrak{m}_{A}\right)$. Then we have to show that for $n \geq 0$

$$
p^{-m} \mathbf{w}_{n}\left(\varphi^{m}(x)\right)=p^{-m} \mathbf{w}_{n+m}(x)=\sum_{i=0}^{n+m} p^{i-m} x_{i}^{p^{n+m-i}} \rightarrow 0
$$

as $m \rightarrow \infty$. For $0 \neq y \in A[1 / p]$, write $v_{p}(y)$ for the greatest integer such that $y p^{-v_{p}(y)} \in A$. Let $a$ be any positive integer. Since $x \in \mathbb{W}\left(\mathfrak{m}_{A}\right),\left\{x_{i}\right\}_{i \geq 1}$ goes to 0 $\mathfrak{m}_{A}$-adically and hence $p$-adically. Thus there exists $i_{0}>0$ such that $v_{p}\left(x_{i}\right)>a$ for $i>i_{0}$. Then also $v_{p}\left(p^{i-m} x_{i}^{p^{n+m-i}}\right)>a$. For $i \leq i_{0}, v_{p}\left(p^{i-m} x_{i}^{p^{n+m-i}}\right)>a$, for $m$ sufficiently large.

3.1.18. Suppose $A=\mathcal{O}_{K}$, where $K / K_{0}$ is a finite, totally ramified extension with uniformizer $\pi$. Then we may apply the previous lemma, and obtain

$$
\mathbb{D} \otimes_{\mathbb{Z}_{p}} \mathbb{Q}_{p} \rightarrow M \otimes_{\mathbb{Z}_{p}} \mathbb{Q}_{p} \rightarrow\left(M / I_{\mathcal{O}_{K}} M\right) \otimes_{\mathbb{Z}_{p}} \mathbb{Q}_{p}
$$

The right hand side is a filtered $K$-vector space, and the composite gives $\mathbb{D} \otimes_{\mathbb{Z}_{p}} \mathbb{Q}_{p}$ the structure of a weakly admissible $\varphi$-module. It is the weakly admissible module corresponding to the $p$-divisible group attached to $M=M_{\mathcal{O}_{K}}$. This is easily deduced from [8], Prop. 5.1.3, using the map $S \rightarrow \widehat{W}\left(\mathcal{O}_{K}\right)$ given by $u \mapsto[\pi]$, where, as in loc. cit, $S$ the $p$-adic completion of $W\left[u, E(u)^{i} / i !_{i \geq 1}\right.$.

\subsection{Deformations with crystalline cycles.}

3.2.1. We continue to use the notation above. For the remainder of $\S 3$, we assume that $k$ is algebraically closed, as this simplifies the discussion. The reader can check that for any $k$, the same results go through after replacing $k$ by a finite extension.

For any ring $A$ and a finite free $A$-module $N$, we denote by $N^{\otimes}$ the direct sum of all $A$-modules which can be formed from $N$ by using the operations of taking tensor products, duals, and symmetric and exterior powers.

Suppose that $\left(s_{\alpha, 0}\right) \subset \mathbb{D}^{\otimes}$ is a collection of $\varphi$-invariant sections whose images in $\mathbb{D}^{\otimes} \otimes_{W} k$ lie in $\mathrm{Fil}^{0}$. Suppose that the pointwise stabilizer of $\left(s_{\alpha, 0}\right)$ is a smooth subgroup $\mathcal{G} \subset \mathrm{GL}(\mathbb{D})$, with $\mathcal{G} \otimes_{\mathbb{Z}_{p}} \mathbb{Q}_{p}$ a connected reductive group $G$. As usual, 
we denote by $\mathcal{G}^{\circ}$ the neutral component of $\mathcal{G}$ which is also a smooth affine group scheme over $\mathbb{Z}_{p}$.

We assume the following conditions hold:

There exists an isomorphism $\varphi^{*}(\mathbb{D}) \cong \mathbb{D}$ taking $s_{\alpha, 0} \otimes 1$ to $s_{\alpha, 0}$.

$$
\mathrm{H}^{1}\left(D^{\times}, \mathcal{G}^{\circ}\right)=\{1\},
$$

where, as before $D^{\times}$denotes the complement of the closed point in $D=\operatorname{Spec} \mathfrak{S}$, where $\mathfrak{S}=W \llbracket u \rrbracket$.

$$
G \subset \mathrm{GL}\left(\mathbb{D} \otimes_{\mathbb{Z}_{p}} \mathbb{Q}_{p}\right) \text { contains the scalars. }
$$

3.2.5. Recall, that we fixed a parabolic subgroup $P \subset \mathrm{GL}(\mathbb{D})$ lifting the parabolic subgroup $P_{0} \subset \mathrm{GL}\left(\mathbb{D} \otimes_{W} k\right)$, corresponding to the filtration on $\mathbb{D} \otimes_{W} k$, and we wrote $\mathrm{M}^{\text {loc }}=\mathrm{GL}(\mathbb{D}) / P$, and $\operatorname{Spf} R=\widehat{\mathrm{M}}^{\text {loc }}$ for the completion of $\mathrm{M}^{\text {loc }}$ along the identity.

Let $K^{\prime} / K_{0}$ be a finite extension, and $y: R \rightarrow K^{\prime}$ a $K^{\prime}$-valued point such that $s_{\alpha, 0} \in \operatorname{Fil}^{0} y^{*}\left(\bar{M}^{\otimes}\right)$. Then the stabilizer of $y^{*}\left(\bar{M}_{1}\right) \subset y^{*}(\bar{M})$ is a parabolic subgroup $P_{y} \subset \mathrm{GL}(\mathbb{D})$, defined over $K^{\prime}$, which is conjugate to $P$, and induced by a $G$-valued cocharacter $\mu_{y}$ [3] Lemma 1.4.5. 4 Let $E \subset K^{\prime}$ be the local reflex field of $\mu_{y}$. That is, $E \supset K_{0}$ is the field of definition of the $G$-conjugacy class of $\mu_{y}$. Then the orbit $G \cdot y \subset(\mathrm{GL}(\mathbb{D}) / P)_{K^{\prime}}$ is defined over $E$. Let $\mathrm{M}_{G}^{\text {loc }}$ be the closure $\mathrm{M}_{G}^{\text {loc }}$ of $G \cdot y \subset(\mathrm{GL}(\mathbb{D}) / P)_{\mathcal{O}_{E}}$. Note that $\mathrm{M}_{G}^{\text {loc }}$ depends only on the $G$-conjugacy class of $\mu_{y}$, and not on $y$.

Let $\bar{M}$ be the vector bundle $\mathbb{D} \otimes_{W} \mathcal{O}_{\mathrm{M}^{1 \text { oc }}}$ on $\mathrm{M}^{\text {loc }}$ and $\bar{M}_{1} \subset \bar{M}$ the subbundle corresponding to universal parabolic subgroup of $\mathrm{GL}(\mathbb{D})$ over $\mathrm{M}^{\mathrm{loc}}$. These restrict to the bundles denoted by the same symbols on $\widehat{\mathrm{M}}^{\text {loc }}$. Since $G$ fixes the $s_{\alpha, 0}$ pointwise, we have $s_{\alpha, 0} \in \operatorname{Fil}^{0} \bar{M}^{\otimes}$ over $\mathrm{M}_{G}^{\text {loc }}$.

We denote by $\widehat{\mathrm{M}}_{G}^{\mathrm{loc}}=\operatorname{Spf} R_{G}$ the completion of $\mathrm{M}_{G}^{\text {loc }}$ along (the image of) the identity in $\operatorname{GL}\left(\mathbb{D} \otimes_{W} k\right)$. Then $\widehat{\mathrm{M}}_{G}^{\text {loc }}$ depends only the $G$-conjugacy class of $\mu_{y}$, and the specialization of $y$ in $(\mathrm{GL}(\mathbb{D}) / P) \otimes k$. By construction $R_{G}$, is a quotient of $R_{E}=R \otimes_{W} \mathcal{O}_{E}$.

We remark that, in the special situation considered in $\S 2$, the definition of $\mathrm{M}_{G}^{\text {loc }}$ given there for $\mu=\mu_{y}^{-1}$ agrees with the one in this section by Proposition 2.3.7 More precisely, if $G$ is defined over $\mathbb{Q}_{p}$, then in $\S 2, \mathrm{M}_{G}^{\text {loc }}$ was defined as a scheme over the integers of the reflex field of $\mu_{y}$ over $\mathbb{Q}_{p}$, and its base change to $\mathcal{O}_{E} \supset W(k)$ is what we denote $\mathrm{M}_{G}^{\mathrm{loc}}$ in the present subsection.

Let $K / K_{0}$, be a totally ramified, finite extension. Let $\pi$ be a uniformizer of $K$, with Eisenstein polynomial $E(u)$. We regard $\mathcal{O}_{K}$ as a $\mathfrak{S}$-algebra via $u \mapsto \pi$. Write $M_{\mathfrak{S}}=\mathbb{D} \otimes_{W} \mathfrak{S}$. Note that this is an exception to our usual convention, for which, for a ring $A, M_{A}$ is a $\widehat{W}(A)$-module.

Lemma 3.2.6. Let $\xi: R_{G} \rightarrow \mathcal{O}_{K}$ be an $\mathcal{O}_{K}$-valued point, and let $F \subset M_{\mathfrak{S}}$ denote the preimage of $\xi^{*}\left(\bar{M}_{1}\right)$. Then $F$ is a free $\mathfrak{S}$-module and

$$
\text { (1) } s_{\alpha, 0} \in F^{\otimes} \subset F^{\otimes}[1 / E(u)]=M_{\mathfrak{S}}^{\otimes}[1 / E(u)] \text {. }
$$

\footnotetext{
${ }^{4}$ The last line of the proof of loc. cit requires a correction, since the group scheme $\mathrm{Aut}^{\otimes}(\omega)$ is not in general reductive, so one cannot apply (1.1.3) to it. One should replace that line by:

Let $\langle D\rangle^{\otimes, G}$ denote the Tannakian category generated by the $G$-representation $D$. By Tannakian duality, $\langle D\rangle^{\otimes}, G$ is a subcategory of $\langle D\rangle^{\otimes}$, and the filtration on $\langle D\rangle^{\otimes} \otimes_{K_{0}} K$ induces a filtration on $\langle D\rangle^{\otimes, G} \otimes_{K_{0}} K$. Hence the filtration on $D \otimes K_{0} K$ is $G$-split by (1.1.3).
} 
(2) The scheme $\underline{\operatorname{Isom}}_{\left(s_{\alpha, 0}\right)}\left(F, M_{\mathfrak{S}}\right)$ consisting of isomorphisms respecting the tensors $\left(s_{\alpha, 0}\right)$, is a trivial $\mathcal{G}$-torsor over $\mathfrak{S}$.

Proof. Since $M_{\mathfrak{S}} / F$ is a free $\mathcal{O}_{K}$-module and $\mathcal{O}_{K}=\mathfrak{S} / E(u) \mathfrak{S}$ has projective dimension 1 over $\mathfrak{S}, F$ is free over $\mathfrak{S}$.

Clearly, the remaining two statements hold over $D[1 / E(u)]=\operatorname{Spec} \mathfrak{S}[1 / E(u)]$ since $F[1 / E(u)]=M_{\mathfrak{S}}[1 / E(u)]$; in fact, $\underline{\operatorname{Isom}}_{\left(s_{\alpha, 0}\right)}\left(\left.F\right|_{D[1 / E(u)]},\left.M_{\mathfrak{S}}\right|_{D[1 / E(u)]}\right)$ is a trivial $\mathcal{G}$-torsor. By [43] Lemma 1.4.5 there exists a $G$-valued cocharacter $\mu$, defined over $K$, such that $\xi^{*}\left(\bar{M}_{1}\right) \subset \xi^{*}(\bar{M})$ is the filtration induced by $\mu$. Let $\widehat{\mathfrak{S}}_{0}$ denote the completion of $\mathfrak{S}[1 / p]$ at the ideal $E(u) \mathfrak{S}$. Then $\widehat{\mathfrak{S}}_{0}$ is a $K$-algebra and $F \otimes_{\mathfrak{S}} \widehat{\mathfrak{S}}_{0}=E(u) \mu(E(u))^{-1} \cdot M_{\widehat{\mathfrak{S}}_{0}}$. Hence $F \otimes_{\mathfrak{S}} \widehat{\mathfrak{S}}_{0}=g \cdot M_{\widehat{\mathfrak{S}}_{0}}$ for some $g \in$ $G\left(\widehat{\mathfrak{S}}_{0}\right)$, since we are assuming that $G$ contains the subgroup of scalars. As the $s_{\alpha, 0}$ are $G$-invariant, this implies that (1) holds over Spec $\widehat{\mathfrak{S}}_{0}$ and hence over $D^{\times}$, and that $\underline{\operatorname{Isom}}_{\left(s_{\alpha, 0}\right)}\left(\left.F\right|_{D^{\times}},\left.M_{\mathfrak{S}}\right|_{D^{\times}}\right)$is a $\mathcal{G}$-torsor. Moreover, we have $s_{\alpha, 0} \in F^{\otimes}=$ $\Gamma\left(D^{\times}, F^{\otimes}\right)$, which proves $(1)$.

Next we show that the $\mathcal{G}$-torsor $\left.\underline{\operatorname{Isom}}_{\left(s_{\alpha, 0}\right)}\left(F, M_{\mathfrak{S}}\right)\right|_{D^{\times}}$can be reduced to a $\mathcal{G}^{\circ}$ torsor. There is an exact sequence of étale sheaves on Spec $W$

$$
1 \rightarrow \mathcal{G}^{\circ} \rightarrow \mathcal{G} \rightarrow i_{*}(\Gamma) \rightarrow 1
$$

where $\Gamma$ is finite (constant) on $\operatorname{Spec}(k)$ and $i: \operatorname{Spec} k \hookrightarrow \operatorname{Spec} W$ is the natural immersion. Taking étale cohomology over $D^{\times}$gives an exact sequence of pointed sets

$$
\mathrm{H}^{1}\left(D^{\times}, \mathcal{G}^{\circ}\right) \rightarrow \mathrm{H}^{1}\left(D^{\times}, \mathcal{G}\right) \rightarrow \mathrm{H}^{1}\left(D^{\times} \otimes_{W} k, \Gamma\right) .
$$

We saw above that the restriction of $\left.\underline{\operatorname{Isom}}_{\left(s_{\alpha, 0}\right)}\left(F, M_{\mathfrak{S}}\right)\right|_{D^{\times}}$to $\operatorname{Spec} \mathfrak{S}[1 / E(u)]$, and hence to $D^{\times} \otimes_{W} k=\operatorname{Spec} k((u))$, is a trivial $\mathcal{G}$-torsor. Thus $\left.\underline{\operatorname{Isom}}_{\left(s_{\alpha, 0}\right)}\left(F, M_{\mathfrak{S}}\right)\right|_{D^{\times}}$ can be reduced to a $\mathcal{G}^{\circ}$-torsor.

Since $\mathrm{H}^{1}\left(D^{\times}, \mathcal{G}^{\circ}\right)=\{1\}$, the torsor $\left.\underline{\operatorname{Isom}}_{\left(s_{\alpha, 0}\right)}\left(F, M_{\mathfrak{S}}\right)\right|_{D^{\times}}$is trivial, and there is an isomorphism $F \cong M_{\mathfrak{S}}$ over $D^{\times}$respecting the $s_{\alpha, 0}$. Such an isomorphism necessarily extends over $D$, which proves the two statements.

Lemma 3.2.9. Let $\xi: R_{G} \rightarrow \mathcal{O}_{K}$. Then

(1) $s_{\alpha, 0} \in \widetilde{M}_{\mathcal{O}_{K}, 1}^{\otimes}=\widetilde{M}_{1} \otimes_{\widehat{W}(R)} \widehat{W}\left(\mathcal{O}_{K}\right)^{\otimes}$.

(2) The scheme

$$
\mathcal{T}_{\xi}=\underline{\operatorname{Isom}}_{\left(s_{\alpha, 0}\right)}\left(\widetilde{M}_{1} \otimes_{\widehat{W}(R)} \widehat{W}\left(\mathcal{O}_{K}\right), M \otimes_{\widehat{W}(R)} \widehat{W}\left(\mathcal{O}_{K}\right)\right)
$$

is a $\mathcal{G}$-torsor over $\widehat{W}\left(\mathcal{O}_{K}\right)$.

Proof. As remarked in 3.1.6, we have

$$
\widetilde{M}_{1} \otimes_{\widehat{W}(R)} \widehat{W}\left(\mathcal{O}_{K}\right)=\widetilde{M}_{\mathcal{O}_{K}, 1} \subset \varphi^{*}\left(\xi^{*}(M)\right)=\varphi^{*}\left(M_{\mathcal{O}_{K}}\right)
$$

so the first statement in (1) makes sense.

Let $\mathfrak{S} \rightarrow \widehat{W}\left(\mathcal{O}_{K}\right)$ be the unique Frobenius equivariant map lifting the identity on $\mathcal{O}_{K}$. This is given by $u \mapsto[\pi]$. Choose a decomposition $M_{\mathfrak{S}}=L \oplus T$ as $\mathfrak{S}$ modules such that $F=L \oplus E(u) T$. Applying $\otimes_{\mathfrak{S}} \widehat{W}\left(\mathcal{O}_{K}\right)$ to $L \oplus T$ gives a normal decomposition of $\left(M_{\mathcal{O}_{K}}, M_{\mathcal{O}_{K}, 1}\right)$.

Note that $E([\pi])$ is not a zero divisor in $\widehat{W}\left(\mathcal{O}_{K}\right)$. To see note this that

$$
\mathbf{w}_{n}(E([\pi]))=\mathbf{w}_{0}\left(\varphi^{n}(E([\pi]))\right)=\mathbf{w}_{0}\left(E\left[\pi^{p^{n}}\right]\right)=E\left(\pi^{p^{n}}\right),
$$


which is non-zero for $n \geq 1$. Thus if $z \in \widehat{W}\left(\mathcal{O}_{K}\right)$ satisfies $z \cdot E([\underline{\pi}])=0$, then $\mathbf{w}_{n}(z)=0$ for $n \geq 1$. But this implies $\mathbf{w}_{n}(\varphi(z))=0$ for $n \geq 0$, so $\varphi(z)=0$ and hence $z=0$. Thus

$$
\begin{aligned}
\varphi^{*}(F) \otimes_{\mathfrak{S}} \widehat{W}\left(\mathcal{O}_{K}\right) & =\varphi^{*}\left(L \otimes_{\mathfrak{S}} \widehat{W}\left(\mathcal{O}_{K}\right) \oplus E(u) T \otimes_{\mathfrak{S}} \widehat{W}\left(\mathcal{O}_{K}\right)\right) \\
= & \varphi^{*}\left(L \otimes_{\mathfrak{S}} \widehat{W}\left(\mathcal{O}_{K}\right)\right)+p \varphi^{*}\left(T \otimes_{\mathfrak{S}} \widehat{W}\left(\mathcal{O}_{K}\right)\right) \cong \widetilde{M}_{\mathcal{O}_{K} \cdot 1} .
\end{aligned}
$$

Thus both parts of the lemma follow from the corresponding statements in Lemma 3.2.6. and the fact that the $s_{\alpha, 0}$ are $\varphi$-invariant.

Corollary 3.2.11. Suppose that $R_{G}$ is normal. Then

$$
s_{\alpha, 0} \in \widetilde{M}_{R_{G}, 1}^{\otimes}=\widetilde{M}_{1}^{\otimes} \otimes_{\widehat{W}(R)} \widehat{W}\left(R_{G}\right)
$$

and

$$
\mathcal{T}=\underline{\operatorname{Isom}}_{\left(s_{\alpha, 0}\right)}\left(\widetilde{M}_{1} \otimes_{\widehat{W}(R)} \widehat{W}\left(R_{G}\right), M \otimes_{\widehat{W}(R)} \widehat{W}\left(R_{G}\right)\right)
$$

is a trivial $\mathcal{G}$-torsor over $\widehat{W}\left(R_{G}\right)$.

Proof. Since $R_{G}$ is normal, it follow from [19] Proposition 7.3.6, that if $s \in R_{G}[1 / p]$ is an element such that $\xi(s) \in \mathcal{O}_{K}$ for every finite extension $K / E$ and $\xi: R_{G} \rightarrow \mathcal{O}_{K}$, then $s \in R_{G}$.

Now suppose $f \in \widehat{W}\left(R_{G}\right)$ is non-zero. Then $f$ is divisible by $p$ in $\widehat{W}\left(R_{G}\right)$ if and only if $\xi(f)$ is divisible by $p$ for every $\xi$ as above. To see this note that $p^{-1} f \in \widehat{W}\left(R_{G}\right)$ if and only certain universal polynomials in $\mathbf{w}_{n}(f), n=0,1,2, \ldots$, with coefficients in $\mathbb{Z}[1 / p]$ take values in $R_{G}$. By what we just saw, this is equivalent to asking that the same polynomials in $\mathbf{w}_{n}(\xi(f))$ take values in $\mathcal{O}_{K}$ for all $\xi$, which is the same as $p^{-1} \xi(f) \in \widehat{W}\left(\mathcal{O}_{K}\right)$. Now the first claim of the Corollary follows from (1) of Lemma 3.2 .9

By Lemma 3.2.9. for every $\xi$ as above, $\xi^{*}(\mathcal{T})$ is a trivial $\mathcal{G}$-torsor. By 65, Thm. 4.1.2] this implies that $\mathcal{T}$ is flat over $\widehat{W}\left(R_{G}\right)$, as $\cap_{\xi} \operatorname{ker}(\widehat{W}(\xi))=0$. Moreover, $\mathcal{T}$ has a non-empty fibre over the closed point of Spec $\widehat{W}\left(R_{G}\right)$. Hence $\mathcal{T}$ is a $\mathcal{G}$-torsor, which is necessarily trivial as $k$ is algebraically closed.

3.2.12. For the remainder of the section we assume that $R_{G}$ is normal, so that the conditions of Corollary $3.2 .11(2)$ are satisfied.

Let $\mathfrak{a}_{R_{E}}=\mathfrak{m}_{R_{E}}^{2}+\pi_{E} R_{E}$, where $\pi_{E} \in \mathcal{O}_{E}$ is a uniformizer. Note that $R_{E} / \mathfrak{a}_{R_{E}}=$ $R / \mathfrak{a}_{R}$. Choose an isomorphism $\Psi_{R_{G}}: \widetilde{M}_{R_{G}, 1} \cong M_{R_{G}}$ which respects the $s_{\alpha, 0}$, and such that $\Psi_{R_{G}}$ is constant modulo $\mathfrak{a}_{R_{E}}$ in the sense that the reduction of $\Psi_{R_{G}}$ modulo $\mathfrak{a}_{R_{E}}$ is induced by the isomorphism $\Psi_{0} \otimes 1$ of 3.1.11] Note that this is possible as $\mathcal{G}$ is smooth, and if $\Psi_{R_{G}}$ is constant modulo $\mathfrak{a}_{R_{E}}$ then the map $\widetilde{M}_{R_{G}, 1} \otimes_{\widehat{W}\left(R_{G}\right)} \widehat{W}\left(R_{G} / \mathfrak{a}_{R_{E}}\right) \rightarrow M_{R_{G} / \mathfrak{a}_{R_{E}}}$ does respect the $s_{\alpha, 0}$. Finally, lift $\Psi_{R_{G}}$ to any isomorphism $\Psi: \widetilde{M}_{R_{E}, 1} \cong M_{R_{E}}$ which is constant $\bmod \mathfrak{a}_{R_{E}}$.

As in 3.1.8 $\left(M_{R_{E}}, \widetilde{M}_{R_{E}, 1}, \Psi\right)$ gives rise to a Dieudonné display over $R_{E}$, and hence to a $p$-divisible group $\mathscr{G}_{R_{E}}$ over $R_{E}$. If $R_{E}^{\prime}$ is a versal deformation $\mathcal{O}_{E}$-algebra for $\mathscr{G}_{0}$, then $\mathscr{G}_{R_{E}}$ is induced by a map $j: R_{E}^{\prime} \rightarrow R_{E}$. Since $R_{E} / \mathfrak{a}_{R_{E}}=R / \mathfrak{a}_{R}$, it follows from Lemma 3.1 .12 that $j$ is an isomorphism $\bmod \mathfrak{m}_{R_{E}^{\prime}}^{2}+\pi_{E}$. Hence $j$ is a surjection, and hence an isomorphism, as both rings are smooth over $\mathcal{O}_{E}$ of the same dimension. In particular, $\mathscr{G}_{R_{E}}$ is versal. 
Lemma 3.2.13. With the notation and assumptions of Lemma 3.1.17, suppose that $t \in \mathbb{D}^{\otimes}$, and $\tilde{t} \in M_{A}^{\otimes}$ are Frobenius invariant with $\tilde{t}$ lifting $t$, and that there is an $W$-linear section $s: \mathbb{D} \rightarrow M_{A}$ sending $t$ to $\tilde{t}$. Then the map of Lemma 3.1 .17

$$
\mathbb{D} \otimes_{\mathbb{Z}_{p}} \mathbb{Q}_{p} \rightarrow M_{A} \otimes_{\mathbb{Z}_{p}} \mathbb{Q}_{p}
$$

sends $t$ to $\tilde{t}$.

Proof. The proof of Lemma3.1.17 shows that the map there is given by a convergent sum

$$
s+\sum_{m=0}^{\infty} \Phi^{m+1} \circ s \circ \Phi_{0}^{-m-1}-\Phi^{m} \circ s \circ \Phi_{0}^{-m} .
$$

Since $t$ and $\tilde{t}$ are Frobenius invariant, this map sends $t$ to $s(t)=\tilde{t}$.

Lemma 3.2.14. Let $K / E$ be a finite extension, $\xi: R_{G} \rightarrow \mathcal{O}_{K}$, and let $M_{\mathcal{O}_{K}}$ be the Dieudonné display over $\mathcal{O}_{K}$ induced by $\xi$. Let $M_{\mathcal{O}_{K}[\epsilon]}$ be any deformation of $M_{\mathcal{O}_{K}}$ to a Dieudonné display over $\mathcal{O}_{K}[\epsilon]$, and let $\tilde{s}_{\alpha}$ denote the image of $s_{\alpha, 0}$ under the map

$$
\mathbb{D}^{\otimes} \otimes_{\mathbb{Z}_{p}} \mathbb{Q}_{p} \rightarrow M_{\mathcal{O}_{K}[\epsilon]}^{\otimes} \otimes_{\mathbb{Z}_{p}} \mathbb{Q}_{p}
$$

given by Lemma 3.1.17. Then the following conditions are equivalent

(1) The deformation $M_{\mathcal{O}_{K}[\epsilon]}$ is induced by a lift $\tilde{\xi}: R_{G} \rightarrow \mathcal{O}_{K}[\epsilon]$ of $\xi$.

(2) Any lift $\tilde{\xi}: R_{E} \rightarrow \mathcal{O}_{K}[\epsilon]$ of $\xi$ which induces $M_{\mathcal{O}_{K}[\epsilon]}$ factors through $R_{G}$.

(3) $\tilde{s}_{\alpha}$ maps to an element $s_{\alpha} \in \operatorname{Fil}^{0}\left(\bar{M}_{\mathcal{O}_{K}[\epsilon]}\right)^{\otimes} \otimes_{\mathbb{Z}_{p}} \mathbb{Q}_{p}$.

(4) $\tilde{s}_{\alpha} \in M_{\mathcal{O}_{K}[\epsilon]}^{\otimes}$, maps to $s_{\alpha} \in \operatorname{Fil}^{0}\left(\bar{M}_{\mathcal{O}_{K}[\epsilon]}\right)^{\otimes}$.

Proof. We first check that (1) implies (4). By construction, $M_{R_{G}}=\mathbb{D} \otimes_{W} \widehat{W}\left(R_{G}\right)$, and under this identification the tensors $s_{\alpha, 0} \in M_{R_{G}}^{\otimes}$ are Frobenius invariant, and their images in $\bar{M}_{R_{G}}^{\otimes}$ lie in $\mathrm{Fil}^{0}$. In particular, if (1) holds, we obtain in this way Frobenius invariant tensors $\tilde{s}_{\alpha}^{\prime} \in M_{\mathcal{O}_{K}[\epsilon]}^{\otimes}$, which map to $\mathrm{Fil}^{0} \bar{M}_{\mathcal{O}_{K}[\epsilon]}^{\otimes}$. To show (4), we have to check $\tilde{s}_{\alpha}^{\prime}=\tilde{s}_{\alpha}$. If $s: \mathbb{D} \rightarrow M_{\mathcal{O}_{K}[\epsilon]}$ denotes the tautological inclusion, then $s$ sends $s_{\alpha, 0}$ to $\tilde{s}_{\alpha}^{\prime}$, so (4) follows by Lemma 3.2 .13

We obviously have (4) implies (3), and (2) implies (1) so it remains to show that (3) implies (2). For this we show that the space of lifts $\tilde{\xi}$ such that (3) holds, is an $\mathcal{O}_{K}$-module, and that its rank is equal to the dimension of $R_{G}[1 / p]$. By [73] Thm 3,4, for any deformation $M_{\mathcal{O}_{K}[\epsilon]}$ of $M_{\mathcal{O}_{K}}$ as a display, there is a Frobenius equivariant identification

$$
M_{\mathcal{O}_{K}[\epsilon]} \cong M_{\mathcal{O}_{K}} \otimes_{\widehat{W}\left(\mathcal{O}_{K}\right)} \widehat{W}\left(\mathcal{O}_{K}[\epsilon]\right)
$$

of the underlying $\widehat{W}\left(\mathcal{O}_{K}[\epsilon]\right)$-modules, and isomorphism classes of deformations correspond bijectively to lifts of $\bar{M}_{\mathcal{O}_{K}, 1} \subset \bar{M}_{\mathcal{O}_{K}}$ to a direct summand $\bar{M}_{\mathcal{O}_{K}[\epsilon], 1} \subset$ $\bar{M}_{\mathcal{O}_{K}[\epsilon]}$.

To see which deformations satisfy the condition in (3), we identify $M_{\mathcal{O}_{K}} \otimes_{\mathbb{Z}_{p}}$ $\mathbb{Q}_{p}$ with $\mathbb{D} \otimes_{W} \widehat{W}\left(\mathcal{O}_{K}\right)[1 / p]$ using the isomorphism of Lemma 3.1.17. Combing this with (3.2.16), $M_{\mathcal{O}_{K}[\epsilon]} \otimes_{\mathbb{Z}_{p}} \mathbb{Q}_{p}$ is Frobenius equivariantly identified with $\mathbb{D} \otimes_{W}$ $\widehat{W}\left(\mathcal{O}_{K}[\epsilon]\right)[1 / p]$. As above, one sees that $s_{\alpha, 0}$ is taken to $\tilde{s}_{\alpha}$ under this identification. In particular, this identifies $\bar{M}_{\mathcal{O}_{K}[\epsilon]} \otimes \mathbb{Q}_{p}$ with $\mathbb{D}_{K[\epsilon]}=\mathbb{D} \otimes_{W} K[\epsilon]$, and gives $\mathbb{D}_{K_{0}[\epsilon]}=$ $\mathbb{D} \otimes_{W} K_{0}[\epsilon]$, the structure of a weakly admissible filtered $\varphi$-module, which is a self extension of $\mathbb{D}_{K_{0}}=\mathbb{D} \otimes_{W} K_{0}$. The filtration on $\mathbb{D}_{K[\epsilon]}$ is obtained by translating 
the constant filtration arising from the filtration on $\mathbb{D}_{K}=\mathbb{D} \otimes_{W} K$, by an element $1+\epsilon h$ for some $h \in \operatorname{End}_{K}\left(\mathbb{D}_{K}\right)$. Let $P_{\xi} \subset \mathrm{GL}\left(\mathbb{D}_{K}\right)$ be the subgroup respecting the filtration on $\mathbb{D}_{K}$, and consider the map

$$
\operatorname{End}_{K_{0}}\left(\mathbb{D}_{K_{0}}\right) \rightarrow\left(\mathbb{D}^{\otimes}\right)_{\alpha} ; \quad g \mapsto g\left(s_{\alpha, 0}\right) .
$$

Since $s_{\alpha, 0}$ is Frobenius invariant and in $\mathrm{Fil}^{0}$, this is a map of weakly admissible, filtered $\varphi$-modules, and hence is strict for filtrations. It follows that if $g\left(s_{\alpha, 0}\right) \in$ Fil $^{0} \mathbb{D}^{\otimes}$ for all $\alpha$, then $g \in \operatorname{Lie} G+\operatorname{Lie} P_{\xi} \subset \operatorname{End}_{K}\left(\mathbb{D}_{K}\right)$. We apply this to the element $h$. If $s_{\alpha, 0} \in(1+\epsilon h) \mathrm{Fil}^{0} \mathbb{D}_{K}^{\otimes}$, we have $h \cdot s_{\alpha, 0} \in \mathrm{Fil}^{0} \mathbb{D}_{K}^{\otimes}$, so $h \in \operatorname{Lie} G+\operatorname{Lie} P_{\xi}$. Thus we may assume $h \in \operatorname{Lie} G$. Conversely, if $h \in \operatorname{Lie} G$ then $s_{\alpha, 0} \in(1+\epsilon h) \mathrm{Fil}^{0} \mathbb{D}_{K}^{\otimes}$.

Thus the set of lifts of $\bar{M}_{\mathcal{O}_{K}, 1} \subset \bar{M}_{\mathcal{O}_{K}}$ to a direct summand $\bar{M}_{\mathcal{O}_{K}[\epsilon], 1} \subset \bar{M}_{\mathcal{O}_{K}[\epsilon]}$ such that $s_{\alpha, 0} \in \mathrm{Fil}^{0} \bar{M}_{\mathcal{O}_{K}[\epsilon]}^{\otimes}$ can be identified with an $\mathcal{O}_{K}$-module of rank equal to

$$
\operatorname{dim} \operatorname{Lie} G /\left(\operatorname{Lie} P_{\xi} \cap \operatorname{Lie} G\right)=\operatorname{dim} R_{G}[1 / p] .
$$

Let $\operatorname{Def}_{G}\left(\xi ; \mathcal{O}_{K}[\epsilon]\right)$ denote the set of lifts of $\xi$ to a map $R_{G} \rightarrow \mathcal{O}_{K}[\epsilon]$, and let $\operatorname{Def}_{G}\left(M_{\mathcal{O}_{K}}, \mathcal{O}_{K}[\epsilon]\right)$ denote the set of isomorphism classes of deformations of $M_{\mathcal{O}_{K}}$ to a Dieudonné display over $\mathcal{O}_{K}[\epsilon]$ satisfying (3). Then we have a map

$$
\operatorname{Def}_{G}\left(\xi ; \mathcal{O}_{K}[\epsilon]\right) \rightarrow \operatorname{Def}_{G}\left(M_{\mathcal{O}_{K}} ; \mathcal{O}_{K}[\epsilon]\right)
$$

which is injective by the versality of $\mathscr{G}_{R_{E}}$, and Lemma 3.1.15 We have just seen that both source and target are $\mathcal{O}_{K}$-modules of the same rank. If $\tilde{\xi}: R_{E} \rightarrow \mathcal{O}_{K}[\epsilon]$ is a lift of $\xi$ inducing $M_{\mathcal{O}_{K}[\epsilon]} \in \operatorname{Def}_{G}\left(M_{\mathcal{O}_{K}} ; \mathcal{O}_{K}[\epsilon]\right)$, this shows that for $n$ large enough, the composite of $\tilde{\xi}$ with the map $\mathcal{O}_{K}[\epsilon] \rightarrow \mathcal{O}_{K}[\epsilon]$ given by $\epsilon \mapsto p^{n} \epsilon$ factors through $R_{G}$. Since this last map is injective, this shows that $\tilde{\xi}$ factors through $R_{G}$ and proves that (3) implies (2).

Proposition 3.2.17. Let $K / E$ be a finite extension, and $\mathscr{G}_{\mathcal{O}_{K}}$ a deformation of $\mathscr{G}_{0}$ satisfying the following conditions.

(1) Under the canonical isomorphism $\mathbb{D}\left(\mathscr{G}_{\mathcal{O}_{K}}\right)\left(\mathcal{O}_{K}\right) \otimes_{\mathcal{O}_{K}} K \cong \mathbb{D} \otimes_{K_{0}} K=\mathbb{D}_{K}$, the filtration on $\mathbb{D}\left(\mathscr{G}_{\mathcal{O}_{K}}\right)\left(\mathcal{O}_{K}\right) \otimes_{\mathcal{O}_{K}} K$ is induced by a $G$-valued cocharacter conjugate to $\mu_{y}$.

(2) The $s_{\alpha, 0}$ lift to Frobenius invariant tensors $\tilde{s}_{\alpha} \in \mathbb{D}\left(\mathscr{G}_{\mathcal{O}_{K}}\right)\left(\widehat{W}\left(\mathcal{O}_{K}\right)\right)^{\otimes}$, and there is an isomorphism

$$
\mathbb{D} \otimes_{W} \widehat{W}\left(\mathcal{O}_{K}\right) \cong \mathbb{D}\left(\mathscr{G}_{\mathcal{O}_{K}}\right)\left(\widehat{W}\left(\mathcal{O}_{K}\right)\right)
$$

taking $s_{\alpha, 0}$ to $\tilde{s}_{\alpha}$.

Then any morphism $\xi: R_{E} \rightarrow \mathcal{O}_{K}$ inducing $\mathscr{G}_{\mathcal{O}_{K}}$ factors through $R_{G}$.

Proof. The map in (2) induces a map

$$
\mathbb{D} \rightarrow \mathbb{D}\left(\mathscr{G}_{\mathcal{O}_{K}}\right)\left(\widehat{W}\left(\mathcal{O}_{K}\right)\right) \otimes_{\widehat{W}\left(\mathcal{O}_{K}\right)} W=\mathbb{D}\left(\mathscr{G}_{0}\right)(W)=\mathbb{D} .
$$

which takes $s_{\alpha, 0}$ to $s_{\alpha, 0}$, hence is given by an element of $\mathcal{G}(W)$. Lifting this element to $\mathcal{G}\left(\widehat{W}\left(\mathcal{O}_{K}\right)\right)$, we may modify the map in $(2)$ and assume it lifts the identity on $\mathbb{D}$. Then Lemma 3.2.13 implies that the $\tilde{s}_{\alpha}$ are the images of $s_{\alpha, 0}$ under the canonical map given by Lemma 3.1.17. Denote by $s_{\alpha} \in \mathbb{D}\left(\mathscr{G}_{\mathcal{O}_{K}}\right)\left(\mathcal{O}_{K}\right)^{\otimes}$ the image of $\tilde{s}_{\alpha}$.

Let $\mathbb{D}_{\mathcal{O}_{K}}=\mathbb{D} \otimes_{W} \mathcal{O}_{K}$, and consider the filtration on $\mathbb{D}_{\mathcal{O}_{K}}$ induced by the isomorphism $\mathbb{D}_{\mathcal{O}_{K}} \cong \mathbb{D}\left(\mathscr{G}_{\mathcal{O}_{K}}\right)\left(\mathcal{O}_{K}\right)$ arising from the map in (2). This map takes $s_{\alpha, 0}$ to $s_{\alpha}$, and hence differs from the isomorphism in (1) by an element of $G(K)$. In particular, the induced filtration on $\mathbb{D}_{\mathcal{O}_{K}}$ corresponds to a parabolic subgroup $G$-conjugate to 
$P_{y}$, so $s_{\alpha} \in \mathrm{Fil}^{0} \mathbb{D}_{\mathcal{O}_{K}}^{\otimes}$. As this filtration lifts the one on $\mathbb{D} \otimes_{W} k$, it corresponds to a point $y^{\prime}: R_{G} \rightarrow \mathcal{O}_{K}$. As $R_{G}$ depends only on the reduction of $y$, and the conjugacy class of $\mu_{y}$, we may assume $y=y^{\prime}$ (and $K^{\prime}=K$ ) in order to simplify notation.

Let $\left(M_{\mathcal{O}_{K}}, M_{\mathcal{O}_{K}, 1}, \Phi_{1}, \Phi\right)$ be the Dieudonné display corresponding to $y$, and $\Psi: \widetilde{M}_{\mathcal{O}_{K}, 1} \cong M_{\mathcal{O}_{K}}$ the isomorphism associated by Lemma 3.1.5. Recall that, by construction, $M_{\mathcal{O}_{K}}$ is identified with $\mathbb{D} \otimes_{W} \widehat{W}\left(\mathcal{O}_{K}\right)$, and $\Psi$ takes $s_{\alpha, 0}$ to $s_{\alpha, 0}$. By what we have just seen, $\mathscr{G}_{\mathcal{O}_{K}}$ arises from a morphism $\Psi^{\prime}: \widetilde{M}_{\mathcal{O}_{K}, 1} \cong M_{\mathcal{O}_{K}}$, which takes $s_{\alpha, 0}$ to $s_{\alpha, 0}$ (because the $\tilde{s}_{\alpha}$ are fixed by Frobenius), and reduces to $\Psi_{0}: \widetilde{\mathbb{D}}_{1} \cong \mathbb{D}$.

We now construct a Dieudonné display over $S=\mathcal{O}_{K} \llbracket T \rrbracket$. First consider the base change of $\left(M_{\mathcal{O}_{K}}, M_{\mathcal{O}_{K}, 1}, \Phi_{1}, \Phi\right)$ to $S,\left(M_{S}, M_{S, 1}, \Phi_{1}, \Phi\right)$. The map $\mathcal{O}_{K} \llbracket T \rrbracket \rightarrow \mathcal{O}_{K} \times_{k}$ $\mathcal{O}_{K}$ given by $T \mapsto(0, \pi)$ is surjective, and hence so is $\widehat{W}\left(\mathcal{O}_{K} \llbracket T \rrbracket\right) \rightarrow \widehat{W}\left(\mathcal{O}_{K}\right) \times_{W}$ $\widehat{W}\left(\mathcal{O}_{K}\right)$. Hence, by Corollary 3.2.11, there exists an isomorphism $\Psi_{S}: \widetilde{M}_{S .1} \cong M_{S}$ which takes $s_{\alpha, 0}$ to $s_{\alpha, 0}$, and specializes to $\left(\Psi, \Psi^{\prime}\right)$ under $T \mapsto(0, \pi)$. We take $M_{S}$ to be the Dieudonné display over $S$ associated to $\Psi_{S}$ by Lemma 3.1.5

Now let $\xi: R_{E} \rightarrow \mathcal{O}_{K}$ be a map inducing $\mathscr{G}_{\mathcal{O}_{K}}$. By versality, we may lift the map $(y, \xi): R_{E} \rightarrow \mathcal{O}_{K} \times_{k} \mathcal{O}_{K}$ to a map $\tilde{\xi}: R_{E} \rightarrow S$ which induces $M_{S}$, and we may identify the Dieudonné display $M_{S}$ with the base change of $M_{R_{E}}$ by $\tilde{\xi}$. We will show that $\tilde{\xi}$ factors through $R_{G}$, which implies that $\xi$ does also.

For $n \geq 1$, let $S_{n}=S / T^{n}$, and denote by $M_{S_{n}}$ the base change of $M_{S}$ to $S_{n}$. Let $I_{n}=\operatorname{ker}\left(R_{E} \stackrel{\tilde{\xi}}{\rightarrow} S \rightarrow S_{n}\right)$, and let $J_{G}=\operatorname{ker}\left(R_{E} \rightarrow R_{G}\right)$. Let $\mathfrak{n}=\operatorname{ker}\left(y: R_{G} \rightarrow \mathcal{O}_{K}\right)$, and $J_{G}^{n}=\operatorname{ker}\left(R_{E} \rightarrow\left(R_{G} / \mathfrak{n}^{n}\right)[1 / p]\right)$. By Lemma 3.2.13, under the canonical map given by Lemma 3.1.17, $s_{\alpha, 0} \in \mathbb{D}^{\otimes}$ is mapped to $s_{\alpha, 0} \in M_{S_{n}}^{\otimes}$. It follows that, for the Dieudonné display $M_{R_{E} / I_{n} \cap J_{G}^{n}}$, the map of Lemma 3.1.17 sends $s_{\alpha, 0}$ to $s_{\alpha, 0}$. In particular, by Lemma 3.2.14, any map $R_{E} \rightarrow \mathcal{O}_{K}[\epsilon]$ which factors through $R_{E} / I_{n} \cap J_{G}^{n}$, factors through $R_{G}$.

Now let $I=\operatorname{ker} \tilde{\xi}$, and $R_{G}^{\prime}=R_{E} / I \cap J_{G}$, and consider a map $\theta: R_{G}^{\prime}[1 / p] \rightarrow K[\epsilon]$ which lifts $y$. After replacing $\epsilon$ by $p^{-n} \epsilon$ we may assume that $\theta$ induces a map $R_{G}^{\prime} \rightarrow \mathcal{O}_{K}[\epsilon]$. We also write $\theta$ for the induced map $R_{E} \rightarrow K[\epsilon]$. Since $\left(I \cap J_{G}\right)[1 / p]=$ $\cap_{n}\left(\left(I_{n} \cap J_{G}^{n}\right)[1 / p]\right)$, and the decreasing sequence of $E$-subspaces $\theta\left(\left(I_{n} \cap J_{G}^{n}\right)[1 / p]\right) \subset$ $\epsilon \cdot K$ must stabilize, we see that $\theta\left(I_{n} \cap J_{G}^{n}\right)=0$ for some $n$. Hence $\theta$ factors through $R_{G}$ by what we saw above. This implies that the tangent spaces of $R_{G}^{\prime}[1 / p]$ and $R_{G}[1 / p]$ at $\mathfrak{n}$ are equal. Since $R_{G}[1 / p]$ is regular, we have

$$
\operatorname{dim}_{\mathfrak{n}} R_{G}^{\prime}[1 / p] \leq \operatorname{dim}_{\kappa(\mathfrak{n})} \mathfrak{n} / \mathfrak{n}^{2}=\operatorname{dim}_{\mathfrak{n}} R_{G}[1 / p] \leq \operatorname{dim}_{\mathfrak{n}} R_{G}^{\prime}[1 / p] .
$$

(Here $\operatorname{dim}_{\mathfrak{n}}$ denotes the dimension at $\mathfrak{n}$.) It follows that $\operatorname{dim}_{\mathfrak{n}} R_{G}^{\prime}[1 / p]=\operatorname{dim}_{\kappa(\mathfrak{n})} \mathfrak{n} / \mathfrak{n}^{2}$, which implies that $R_{G}^{\prime}[1 / p]$ is regular at $\mathfrak{n}$, and $R_{G, \mathfrak{n}}^{\prime}[1 / p]=R_{G, \mathfrak{n}}[1 / p]$. In particular,

$$
J_{G} \otimes_{\mathbb{Z}_{p}} \mathbb{Q}_{p} \subset I_{n} \otimes_{\mathbb{Z}_{p}} \mathbb{Q}_{p}
$$

so $J_{G} \subset\left(I_{n} \otimes_{\mathbb{Z}_{p}} \mathbb{Q}_{p}\right) \cap R_{E}=I_{n}$, as $R_{E} / I_{n}$ is $p$-torsion free. Finally, $J_{G} \subset \cap_{n} I_{n}=I$, so $\tilde{\xi}$ factors through $R_{G}$ and so does $\xi$.

\subsection{Deformations with étale cycles.}

3.3.1. We continue to use the notation above, so in particular $k$ is algebraically closed. Set $\Gamma_{K}=\operatorname{Gal}(\bar{K} / K)$. Denote by $\operatorname{Rep}_{\Gamma_{K}}^{\text {cris }}$ the category of crystalline $\Gamma_{K^{-}}$ representations, and by $\operatorname{Rep}_{\Gamma_{K}}^{\text {criso }}$ the category of $\Gamma_{K}$-stable $\mathbb{Z}_{p}$-lattices spanning 
a representation in $\operatorname{Rep}_{\Gamma_{K}}^{\text {cris }}$. For $V$ a crystalline representation, recall Fontaine's functors

$$
D_{\text {cris }}(V)=\left(B_{\text {cris }} \otimes_{\mathbb{Q}_{p}} V\right)^{\Gamma_{K}} \quad \text { and } \quad D_{\mathrm{dR}}(V)=\left(B_{\mathrm{dR}} \otimes_{\mathbb{Q}_{p}} V\right)^{\Gamma_{K}} .
$$

Fix a uniformiser $\pi \in K$, and let $E(u) \in W[u]$ be the Eisenstein polynomial for $\pi$. We have the $\varphi$-equivariant inclusion $\mathfrak{S} \hookrightarrow \widehat{W}\left(\mathcal{O}_{K}\right)$ introduced above. As above, we denote by $D^{\times}$the complement of the closed point in Spec $\mathfrak{S}$.

Let $\operatorname{Mod}_{/ \mathfrak{S}}^{\varphi}$ denote the category of finite free $\mathfrak{S}$-modules $\mathfrak{M}$ equipped with a Frobenius semi-linear isomorphism

$$
1 \otimes \varphi: \varphi^{*}(\mathfrak{M})[1 / E(u)] \cong \mathfrak{M}[1 / E(u)] .
$$

For $\mathfrak{M} \in \operatorname{Mod}_{/ \mathfrak{S}}^{\varphi}$ and $i$ an integer, we set

$$
\operatorname{Fil}^{i} \varphi^{*}(\mathfrak{M})=\varphi^{*}(\mathfrak{M}) \cap(1 \otimes \varphi)^{-1}\left(E(u)^{i} \mathfrak{M}\right) .
$$

If we view $K$ as a $\mathfrak{S}$-algebra via $u \mapsto \pi$, then this induces a filtration on $\varphi^{*}(\mathfrak{M}) \otimes_{\mathfrak{S}} K$.

Theorem 3.3.2. There exists a fully faithful tensor functor

$$
\mathfrak{M}: \operatorname{Rep}_{\Gamma_{K}}^{\text {criso }} \rightarrow \operatorname{Mod}_{/ \mathfrak{S}}^{\varphi},
$$

which is compatible with formation of symmetric and exterior powers, and such that $\left.L \mapsto \mathfrak{M}(L)\right|_{D^{\times}}$is exact. If $L$ is in $\operatorname{Rep}_{\Gamma_{K}}^{\text {criso }}, V=L \otimes_{\mathbb{Z}_{p}} \mathbb{Q}_{p}$, and $\mathfrak{M}=\mathfrak{M}(L)$, then

(1) There are canonical isomorphisms

$$
D_{\text {cris }}(V) \cong \mathfrak{M} / u \mathfrak{M}[1 / p] \text { and } D_{\mathrm{dR}}(V) \cong \varphi^{*}(\mathfrak{M}) \otimes_{\mathfrak{S}} K
$$

where the first isomorphism is compatible with Frobenius, and the second isomorphism is compatible with filtrations.

(2) If $L=T_{p} \mathscr{G}^{\vee}:=\operatorname{Hom}_{\mathbb{Z}_{p}}\left(T_{p} \mathscr{G}, \mathbb{Z}_{p}\right)$ for a $p$-divisible group $\mathscr{G}$ over $\mathcal{O}_{K}$, then there is a canonical isomorphism

$$
\mathbb{D}(\mathscr{G})\left(\widehat{W}\left(\mathcal{O}_{K}\right)\right) \cong \widehat{W}\left(\mathcal{O}_{K}\right) \otimes_{\mathfrak{S}, \varphi} \mathfrak{M}
$$

such that the induced map

$$
\mathbb{D}(\mathscr{G})\left(\mathcal{O}_{K}\right) \cong \mathcal{O}_{K} \otimes_{\mathfrak{S}} \varphi^{*}(\mathfrak{M}) \rightarrow D_{\mathrm{dR}}\left(T_{p} \mathscr{G}^{\vee}\right)
$$

is compatible with filtrations. In particular, if $\mathscr{G}_{0}=\mathscr{G} \otimes k$ then $\mathbb{D}\left(\mathscr{G}_{0}\right)(W)$ is canonically identified with $\varphi^{*}(\mathfrak{M} / u \mathfrak{M})$.

Proof. Except for the claim that $\left.L \mapsto \mathfrak{M}(L)\right|_{D^{\times}}$is exact, this follows from 43$]$ 1.2.1, 1.4.25. More precisely, let $S$ be the $p$-adic completion of $W\left[u, E(u)^{i} / i\right]_{i \geq 1}$. Then the first isomorphism in (2) is constructed in loc. cit. with $S$ in place of $\widehat{W}\left(\mathcal{O}_{K}\right)$, and we obtain the isomorphism in (2) using the continuous extension $S \hookrightarrow \widehat{W}\left(\mathcal{O}_{K}\right)$ of $\mathfrak{S} \rightarrow \widehat{W}\left(\mathcal{O}_{K}\right)$.

To see the exactness of $\left.L \mapsto \mathfrak{M}(L)\right|_{D^{\times}}$, let $L^{\bullet}$ be an exact sequence in $\operatorname{Rep}_{\Gamma_{K}}^{\text {criso }}$. We have to show that $\left.\mathfrak{M}\left(L^{\bullet}\right)\right|_{D^{\times}}$is exact. Let $Q$ be a cohomology group of $\left.\mathfrak{M}\left(L^{\bullet}\right)\right|_{D^{\times}}$.

By (1) the support of $Q$ on $D^{\times} \otimes_{\mathbb{Z}_{p}} \mathbb{Q}_{p}$ is disjoint from the ideal $E(u)$, and in particular is contained in a finite number of closed points. There is an isomorphism $\varphi^{*}(Q)[1 / E(u)] \cong Q[1 / E(u)]$, which implies that the support of $Q$ on $D^{\times} \otimes_{\mathbb{Z}_{p}} \mathbb{Q}_{p}$ is empty. Finally, the support of $Q$ does not contain the ideal $(p)$, by [43] 1.2.1(2), so $Q=0$.

\footnotetext{
${ }^{5}$ Note also that $T_{p} \mathscr{G}^{*}$ should be replaced by the linear dual $T_{p} \mathscr{G}^{\vee}$ of $T_{p} \mathscr{G}$ in (1.4.2), (1.4.3) and (1.5.11) of the published version of loc. cit.
} 
3.3.3. Suppose that $L$ is in $\operatorname{Rep}_{\Gamma_{K}}^{\text {criso }}$, and let $s_{\alpha \text {,ét }} \in L^{\otimes}$ be a collection of $\Gamma_{K^{-}}$ invariant tensors, which define a subgroup $\mathcal{G} \subset \mathrm{GL}(L)$, which is smooth over $\mathbb{Z}_{p}$ with reductive generic fibre $G$. Applying the functor $\mathfrak{M}$ we obtain corresponding tensors $\tilde{s}_{\alpha} \in \mathfrak{M}(L)^{\otimes}$.

Note that, since the $s_{\alpha \text {,ét }}$ are $\Gamma_{K}$-invariant, the action $\Gamma_{K}$ on $L$ gives rise to a representation

$$
\rho: \Gamma_{K} \rightarrow \mathcal{G}\left(\mathbb{Q}_{p}\right) .
$$

Recall the Kottwitz homomorphism $\kappa_{G}: G\left(K_{0}\right) \rightarrow \pi_{1}(G)_{I}$, where $I=\operatorname{Gal}\left(\bar{K} / K_{0}\right)$. We have the following Lemma, due to Wintenberger.

Proposition 3.3.4. (71]) The image of the crystalline representation

$$
\rho: \Gamma_{K} \rightarrow G\left(\mathbb{Q}_{p}\right)
$$

is contained in $\operatorname{ker} \kappa_{G}$.

Proof. This is proved in [71, Lemme 1] when $K=K_{0}$, however the proof there goes over verbatim without this assumption. Note that we are using here that $k$ is algebraically closed.

Lemma 3.3.5. Suppose that $\mathrm{H}^{1}\left(D^{\times}, \mathcal{G}^{\circ}\right)=\{1\}$, and $\rho$ factors through $\mathcal{G}^{\circ}\left(\mathbb{Z}_{p}\right)$. Then there is an isomorphism

$$
L \otimes_{\mathbb{Z}_{p}} \mathfrak{S} \stackrel{\sim}{\rightarrow} \mathfrak{M}(L)
$$

taking $s_{\alpha}$ to $\tilde{s}_{\alpha}$.

Proof. Write $\mathcal{O}_{\mathcal{G}^{\circ}}=\lim _{i \in J} L_{i}$ with $L_{i} \subset \mathcal{O}_{\mathcal{G}^{\circ}}$ of finite $\mathbb{Z}_{p}$-rank and $\mathcal{G}^{\circ}$-stable, as in 9], Lemma 3.1. Let $\mathfrak{M}\left(\mathcal{O}_{\mathcal{G}^{\circ}}\right):=\lim _{\rightarrow} \mathfrak{M}\left(L_{i}\right)$. Since $\left.L \mapsto \mathfrak{M}(L)\right|_{D^{\times}}$is an exact faithful tensor functor by Theorem 3.3.2. it follows from [9], Thm. 4.3, that $\left.\mathfrak{M}\left(\mathcal{O}_{\mathcal{G}^{\circ}}\right)\right|_{D^{\times}}$is a sheaf of algebras on $D^{\times}$and that $\mathcal{P}^{\circ}=\operatorname{Spec}\left(\mathfrak{M}\left(\mathcal{O}_{\mathcal{G}^{\circ}}\right)\right)_{\mid D^{\times}}$is naturally a $\mathcal{G}^{\circ}$-torsor. If we carry out the same construction with $\mathcal{G}$ in place of $\mathcal{G}^{\circ}$ we obtain a $\mathcal{G}$-torsor $\mathcal{P}$ over $D^{\times}$. By construction, there is a $\mathcal{G}^{\circ}$-equivariant map $\mathcal{P}^{\circ} \rightarrow \mathcal{P}$, so $\mathcal{P}$ is obtained from $\mathcal{P}^{\circ}$ by pushing out by $\mathcal{G}^{\circ} \rightarrow \mathcal{G}$. Our assumptions imply that $\mathcal{P}^{\circ}$ is trivial and hence so is $\mathcal{P}$.

Now let $\mathcal{P}^{\prime} \subset \underline{\operatorname{Hom}}\left(L \otimes_{\mathbb{Z}_{p}} \mathfrak{S}, \mathfrak{M}(L)\right)$ be the scheme of isomorphisms $L \otimes_{\mathbb{Z}_{p}} \mathfrak{S} \cong$ $\mathfrak{M}(L)$ taking $s_{\alpha}$ to $\tilde{s}_{\alpha}$. By [9], Thm. 4.5 there is a natural isomorphism $\mathfrak{M}(L) \cong$ $\mathcal{G} \backslash \mathcal{P} \times L$ where $\mathcal{G}$ acts on $\mathcal{P} \times L$ via $g \cdot(p, e)=\left(p g^{-1}, g e\right)$. This implies that there is a $\mathcal{G}$-equivariant inclusion $\left.\mathcal{P} \subset \mathcal{P}^{\prime}\right|_{D^{\times}}$, so $\left.\mathcal{P}^{\prime}\right|_{D^{\times}}=\mathcal{P}$ is a trivial $\mathcal{G}$-torsor. Hence $\mathcal{P}^{\prime}$ has a section over $D^{\times}$, and the resulting isomorphism necessarily extends to Spec $\mathfrak{S}$.

Corollary 3.3.6. Suppose that $G$ splits over a tamely ramified extension, and has no factors of type $E_{8}$, and that $\mathcal{G}=\mathcal{G}_{x}$ for some $x \in \mathcal{B}\left(G, \mathbb{Q}_{p}\right)$, so that $\mathcal{G}^{\circ}$ is a parahoric group scheme. Then there is an isomorphism

$$
L \otimes_{\mathbb{Z}_{p}} \mathfrak{S} \stackrel{\sim}{\longrightarrow} \mathfrak{M}(L)
$$

taking $s_{\alpha}$ to $\tilde{s}_{\alpha}$.

Proof. By 38 Prop. 3, $\mathcal{G}^{\circ}\left(\mathbb{Z}_{p}\right)=\mathcal{G}\left(\mathbb{Z}_{p}\right) \cap$ ker $\kappa_{G}$. Hence, by Proposition 3.3.4 the action of $\Gamma_{K}$ on $L$ factors through $\mathcal{G}^{\circ}\left(\mathbb{Z}_{p}\right)$. Moreover, $\mathrm{H}^{1}\left(D^{\times}, \mathcal{G}^{\circ}\right)=\{1\}$, by Proposition 1.4.3, so the Corollary follows from Lemma 3.3.5 
3.3.7. Keep the assumptions introduced in (3.3.3). Suppose that $L=T_{p} \mathscr{G}^{\vee}$, where $\mathscr{G}$ is a $p$-divisible group over $\mathcal{O}_{K}$ with special fibre $\mathscr{G}_{0}$. We denote by $s_{\alpha, 0} \in$

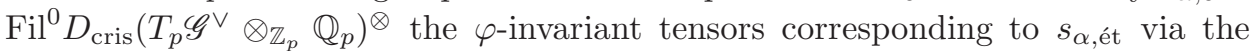
$p$-adic comparison isomorphism.

Assume from now on that $\mathcal{G}=\mathcal{G}_{x}$ for some $x \in \mathcal{B}\left(G, \mathbb{Q}_{p}\right)$, and that $G$ splits over tamely ramified extension, and has no factors of type $E_{8}$.

Proposition 3.3.8. We have $s_{\alpha, 0} \in \mathbb{D}\left(\mathscr{G}_{0}\right)(W)^{\otimes}$, where we view $\mathbb{D}\left(\mathscr{G}_{0}\right)(W) \subset$ $D_{\text {cris }}\left(T_{p} \mathscr{G}^{\vee} \otimes_{\mathbb{Z}_{p}} \mathbb{Q}_{p}\right)$ via the isomorphisms of Theorem 3.3.2, and the $s_{\alpha, 0}$ lift to $\varphi$-invariant tensors $\tilde{s}_{\alpha} \in \mathbb{D}(\mathscr{G})\left(\widehat{W}\left(\mathcal{O}_{K}\right)\right)$ which map into $\mathrm{Fil}^{0} \mathbb{D}(\mathscr{G})\left(\mathcal{O}_{K}\right)^{\otimes}$.

There exists an isomorphism

$$
\mathbb{D}(\mathscr{G})\left(\widehat{W}\left(\mathcal{O}_{K}\right)\right) \cong \widehat{W}\left(\mathcal{O}_{K}\right) \otimes_{\mathbb{Z}_{p}} T_{p} \mathscr{G}^{\vee}
$$

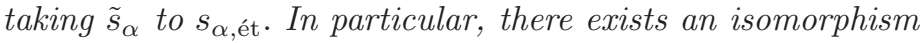

$$
\mathbb{D}\left(\mathscr{G}_{0}\right)(W) \cong W \otimes_{\mathbb{Z}_{p}} T_{p} \mathscr{G}^{\vee}
$$

taking $s_{\alpha, 0}$ to $s_{\alpha, \text { ét }}$.

Proof. Let $\mathfrak{M}=\mathfrak{M}\left(T_{p} \mathscr{G}^{\vee}\right)$, and $\tilde{s}_{\alpha} \in \mathfrak{M}^{\otimes}$ the tensors corresponding to $s_{\alpha \text {,ét. We }}$ may view $\tilde{s}_{\alpha}$ in $\mathbb{D}(\mathscr{G})\left(\widehat{W}\left(\mathcal{O}_{K}\right)\right)^{\otimes}$ via the isomorphism

$$
\mathbb{D}(\mathscr{G})\left(\widehat{W}\left(\mathcal{O}_{K}\right)\right) \cong \widehat{W}\left(\mathcal{O}_{K}\right) \otimes_{\varphi, \mathfrak{S}} \mathfrak{M}
$$

of Theorem 3.3.2 which also implies that these elements specialize to $s_{\alpha, 0} \in$ $\mathbb{D}\left(\mathscr{G}_{0}\right)(W)^{\otimes}$, and map into $\mathrm{Fil}^{0} \mathbb{D}(\mathscr{G})\left(\mathcal{O}_{K}\right)^{\otimes}$.

By Proposition 3.3.6 there is an isomorphism $\mathfrak{M} \cong T_{p} \mathscr{G}^{\vee} \otimes_{\mathbb{Z}_{p}} \mathfrak{S}$ taking $\tilde{s}_{\alpha}$ to $s_{\alpha}$, and the remaining statements in the lemma follow from the isomorphism $\mathbb{D}(\mathscr{G})\left(\widehat{W}\left(\mathcal{O}_{K}\right)\right) \cong \widehat{W}\left(\mathcal{O}_{K}\right) \otimes_{\varphi, \mathfrak{S}} \mathfrak{M}$.

3.3.9. Set $\mathbb{D}=\mathbb{D}\left(\mathscr{G}_{0}\right)(W)$. By Proposition 3.3.8, we may identify the subgroup $\mathcal{G}_{W} \subset \mathrm{GL}(\mathbb{D})$ defined by the $s_{\alpha, 0}$ with $\mathcal{G} \otimes_{\mathbb{Z}_{p}} W$. This identification is independent of the choice of isomorphism $\mathbb{D} \cong W \otimes_{\mathbb{Z}_{p}} T_{p} \mathscr{G}^{\vee}$ up to $\mathcal{G}_{W}$-conjugacy. We write $G_{K_{0}}=\mathcal{G}_{W} \otimes_{W} K_{0}$.

Corollary 3.3.10. With the above assumptions and notation, let $s_{\alpha} \in \mathbb{D}(\mathscr{G})\left(\mathcal{O}_{K}\right)^{\otimes}$ denote the image of $\tilde{s}_{\alpha}$. Then there exists an isomorphism

$$
\mathbb{D}(\mathscr{G})\left(\mathcal{O}_{K}\right) \cong \mathbb{D} \otimes_{W} \mathcal{O}_{K}
$$

taking $s_{\alpha}$ to $s_{\alpha_{0}}$ and lifting the identity on $\mathbb{D}\left(\mathscr{G}_{0}\right)(k)$. In particular, there is a $G_{K_{0}}$ valued cocharacter $\mu_{y}$ such

(1) The filtration on $\mathbb{D} \otimes_{W} K$ induced by the canonical isomorphism

$$
\mathbb{D} \otimes_{W} K \cong \mathbb{D}(\mathscr{G})\left(\mathcal{O}_{K}\right) \otimes_{\mathcal{O}_{K}} K
$$

is given by a $G_{K_{0}}$-valued cocharacter $G_{K_{0}}$-conjugate to $\mu_{y}$.

(2) $\mu_{y}$ induces a filtration on $\mathbb{D}$ which lifts the filtration on $\mathbb{D} \otimes_{W} k=\mathbb{D}\left(\mathscr{G}_{0}\right)(k)$.

Proof. By Proposition 3.3.8 there is an isomorphism $i: \mathbb{D}(\mathscr{G})\left(\mathcal{O}_{K}\right) \cong \mathbb{D} \otimes_{W} \mathcal{O}_{K}$ taking $s_{\alpha}$ to $s_{\alpha, 0}$. Since the scheme of such isomorphisms forms a $\mathcal{G}_{W}$-torsor, we may assume that this isomorphism lifts the identity on $\mathbb{D}\left(\mathscr{G}_{0}\right)(k)$, and we consider the induced filtration on $\mathbb{D} \otimes_{W} \mathcal{O}_{K}$. As above, since $s_{\alpha} \in \mathrm{Fil}^{0} \mathbb{D}(\mathscr{G})\left(\mathcal{O}_{K}\right)^{\otimes}$, this filtration is given by a $G_{K_{0}}$-valued cocharacter $\mu_{y}$, which satisfies (2) by construction.

As $i$ differs from the canonical map $\mathbb{D} \otimes_{W} K \cong \mathbb{D}(\mathscr{G})\left(\mathcal{O}_{K}\right) \otimes_{\mathcal{O}_{K}} K$ by the action of an element of $G_{K_{0}}(K), \mu_{y}$ satisfies (1). 
3.3.11. Keep the assumptions above. We apply the construction of sections 3.1 .8 and 3.2 to the $p$-divisible group $\mathscr{G}_{0}$ equipped with the tensors $s_{\alpha, 0}$. Thus $P_{0} \subset$ $\mathrm{GL}\left(\mathbb{D} \otimes_{W} k\right)$ is a parabolic corresponding to the filtration on $\mathbb{D} \otimes_{W} k$, and $P \subset \mathrm{GL}(\mathbb{D})$ a lifting of $P_{0}$. The filtration in Corollary 3.3 .10 is given by a point $y \in \mathrm{GL}(\mathbb{D}) / P$, which reduces to $P_{0}$, and we have the formal completions of the local models

$$
\widehat{\mathrm{M}}^{\mathrm{loc}}=\widehat{\mathrm{M}}_{y}^{\mathrm{loc}}=\operatorname{Spf} R, \quad \text { and } \quad \widehat{\mathrm{M}}_{G}^{\mathrm{loc}}=\widehat{\mathrm{M}}_{G, y}^{\mathrm{loc}}=\operatorname{Spf} R_{G},
$$

defined over $\mathcal{O}_{E}$, corresponding to the orbit $G \cdot y \subset(\mathrm{GL}(\mathbb{D}) / P)_{\mathcal{O}_{K}}$ which is defined over the reflex field $E / K_{0}$ of $\mu_{y}$.

Note that, by Proposition 3.3.8 $\left(\mathbb{D},\left(s_{\alpha}\right)\right)$ satisfies the condition (3.2.2). As $\mathcal{G}_{W}=\mathcal{G} \otimes_{\mathbb{Z}_{p}} W$, we have $\mathrm{H}^{1}\left(D^{\times}, \mathcal{G}_{W}^{\circ}\right)=\{1\}$, and (3.2.3) is satisfied. We also assume from now on that

$$
R_{G} \text { is normal and } G \text { contains the scalars. }
$$

Then the assumptions in $3.2 .12(2)$ are satisfied, and we may fix an isomorphism $\Psi: \widetilde{M}_{1} \cong M$ lifting $\Psi_{0}$, such that $\Psi$ is constant modulo $\mathfrak{a}_{R_{E}}$, and such that its base change $\Psi_{R_{G}}$ to $\widehat{W}\left(R_{G}\right)$ respects the $s_{\alpha, 0}$.

Proposition 3.3.13. Let $\mathscr{G}^{\prime}$ be a deformation of $\mathscr{G}_{0}$ defined over some finite extension $K / E$ such that

(1) The filtration on $\mathbb{D} \otimes K_{0} K$ corresponding to $\mathscr{G}^{\prime}$ is given by a $G$-valued cocharacter which is $G$-conjugate to $\mu_{y}$.

(2) There exists Galois invariant tensors $s_{\alpha, \text { ét }}^{\prime} \in\left(T_{p} \mathscr{G}^{\prime \vee v}\right)^{\otimes}$ which correspond to $s_{\alpha, 0}$ under the $p$-adic comparison isomorphism.

Then any morphism $R_{E} \rightarrow \mathcal{O}_{K}$ which induces $\mathscr{G}^{\prime}$ factors through $R_{G}$.

Proof. By Lemma 3.3.8, $\mathscr{G}^{\prime}$ satisfies the conditions (1) and (2) of Proposition3.2.17 which implies the present Proposition.

\section{SHIMURA VARIETIES AND LOCAL MODELS}

\subsection{Shimura varieties of Hodge type.}

4.1.1. Let $G$ be a connected reductive group over $\mathbb{Q}$ and $X$ a conjugacy class of maps of algebraic groups over $\mathbb{R}$

$$
h: \mathbb{S}=\operatorname{Res}_{\mathbb{C} / \mathbb{R}} \mathbb{G}_{m} \rightarrow G_{\mathbb{R}},
$$

such that $(G, X)$ is a Shimura datum $[20, \S 2.1$.

For any $\mathbb{C}$-algebra $R$, we have $R \otimes_{\mathbb{R}} \mathbb{C}=R \times c^{*}(R)$ where $c$ denotes complex conjugation, and we denote by $\mu_{h}$ the cocharacter given on $R$-points by

$$
R^{\times} \rightarrow\left(R \times c^{*}(R)\right)^{\times}=\left(R \otimes_{\mathbb{R}} \mathbb{C}\right)^{\times}=\mathbb{S}(R) \stackrel{h}{\rightarrow} G_{\mathbb{C}}(R) .
$$

We set $w_{h}=\mu_{h}^{-1} \mu_{h}^{c-1}$.

Let $\mathbb{A}_{f}$ denote the finite adeles over $\mathbb{Q}$, and $\mathbb{A}_{f}^{p} \subset \mathbb{A}_{f}$ the subgroup of adeles with trivial component at $p$. Let $\mathrm{K}=\mathrm{K}_{p} \mathrm{~K}^{p} \subset G\left(\mathbb{A}_{f}\right)$ where $\mathrm{K}_{p} \subset G\left(\mathbb{Q}_{p}\right)$, and $\mathrm{K}^{p} \subset G\left(\mathbb{A}_{f}^{p}\right)$ are compact open subgroups.

If $\mathrm{K}^{p}$ is sufficiently small then

$$
\operatorname{Sh}_{\mathfrak{K}}(G, X)_{\mathbb{C}}=G(\mathbb{Q}) \backslash X \times G\left(\mathbb{A}_{f}\right) / \mathrm{K}
$$

has a natural structure of an algebraic variety over $\mathbb{C}$, which has a model, $\operatorname{Sh}_{\mathbb{K}}(G, X)$ over a number field $\mathrm{E}=E(G, X)$, which is the minimal field of definition of the 
conjugacy class of $\mu_{h}$. We will always assume in the following that $\mathrm{K}^{p}$ is sufficiently small that the quotient above exists as an algebraic variety.

We will sometimes consider the E-schemes

$$
\operatorname{Sh}(G, X)=\lim _{\leftarrow} \operatorname{Sh}_{\kappa}(G, X),
$$

and

$$
\operatorname{Sh}_{\kappa_{p}}(G, X)=\lim _{\leftarrow} \operatorname{Sh}_{\mathcal{K}}(G, X)
$$

where $\mathrm{K}$ runs through all compact open subgroups in the first limit and through all compact open subgroups with a fixed factor $\mathrm{K}_{p}$ at $p$ in the second limit. These exist as the transition maps are finite, hence affine.

4.1.2. Fix a $\mathbb{Q}$-vector space $V$ with a perfect alternating pairing $\psi$. For any $\mathbb{Q}$ algebra $R$, we write $V_{R}=V \otimes_{\mathbb{Q}} R$. Let $\operatorname{GSp}=\operatorname{GSp}(V, \psi)$ be the corresponding group of symplectic similitudes, and let $S^{ \pm}$be the Siegel double space, defined as the set of maps $h: \mathbb{S} \rightarrow \mathrm{GSp}_{\mathbb{R}}$ such that

(1) The $\mathbb{C}^{\times}$-action on $V_{\mathbb{R}}$ gives rise to a Hodge structure of type $(-1,0),(0,-1)$ :

$$
V_{\mathbb{C}} \cong V^{-1,0} \oplus V^{0,-1} \text {. }
$$

(2) $(x, y) \mapsto \psi(x, h(i) y)$ is (positive or negative) definite on $V_{\mathbb{R}}$.

4.1.3. For the rest of this subsection we will assume that there is an embedding of Shimura data $\iota:(G, X) \hookrightarrow\left(\mathrm{GSp}, S^{ \pm}\right)$. We will sometimes write $G$ for $G_{\mathbb{Q}_{p}}=$ $G \otimes_{\mathbb{Q}} \mathbb{Q}_{p}$, when there is no risk of confusion. We will assume from now on that the following conditions hold

(4.1.4) $G$ splits over a tamely ramified extension of $\mathbb{Q}_{p}$ and that $p \nmid\left|\pi_{1}\left(G^{\mathrm{der}}\right)\right|$.

Fix $x \in \mathcal{B}\left(G, \mathbb{Q}_{p}\right)$ and let $\mathcal{G}=\mathcal{G}_{x}$ be the smooth $\mathbb{Z}_{p}$-group scheme with generic fibre $G$, which is the stabilizer of $x$, so that $\mathcal{G}^{\circ}$ is a parahoric group scheme.

4.1.5. The table 20] 1.3 .9 shows that the symplectic representation $\iota$ is minuscule. In 1.2 we constructed a toral embedding $\mathcal{B}\left(G, \mathbb{Q}_{p}\right) \rightarrow \mathcal{B}\left(\mathrm{GSp}, \mathbb{Q}_{p}\right)$ associated to $\iota$. For simplicity, we again denote by $\iota$ this embedding of buildings. Let $\mathcal{G S P}$ be the smooth $\mathbb{Z}_{p}$-group scheme defined by $\iota(x)$, and let $V_{\mathbb{Z}_{p}} \subset V_{\mathbb{Q}_{p}}$ be the $\mathbb{Z}_{p}$-lattice corresponding to the image of $x$ in $\mathcal{B}\left(\mathrm{GL}\left(V_{\mathbb{Q}_{p}}\right), \mathbb{Q}_{p}\right)$.

By Lemma 1.3.3 $\iota$ induces a closed embedding of $\mathbb{Z}_{p}$-group schemes $\mathcal{G} \hookrightarrow \mathcal{G S P}$. By the discussion in (2.3.15) and Corollary 2.3.16 after replacing $\iota$ by another symplectic embedding, we may and do assume that $\mathcal{G S P}$ is the group scheme corresponding to a lattice $V_{\mathbb{Z}_{p}} \subset V_{\mathbb{Q}_{p}}$ such that $V_{\mathbb{Z}_{p}} \subset V_{\mathbb{Z}_{p}}^{\vee}$, and that $\iota$ induces an embedding of local models $\mathrm{M}_{G, X}^{\text {loc }} \hookrightarrow \mathrm{M}_{\mathrm{GSp}, S^{ \pm}}^{\text {loc }}$.

These models have a more concrete description: Let $P_{h^{-1}} \subset \mathrm{GL}\left(V_{\mathbb{Z}_{p}}\right)$ be a parabolic defined over $\mathbb{Z}_{p}$, and corresponding to a cocharacter in the conjugacy class of $\mu_{h}^{-1}$ for $h \in X$. Let $\mu$ be a $G$-valued cocharacter, defined over $\overline{\mathbb{Q}}_{p}$, and in the $G$-conjugacy class of $\mu_{h}$. The orbit $G \cdot y \subset \operatorname{GL}\left(V_{\mathbb{Z}_{p}}\right) / P_{h^{-1}}$, where $y$ is the filtration defined by $\mu^{-1}$, depends only on $X$ and not on the choice of $\mu$, and is

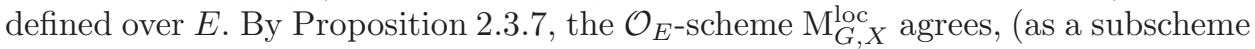
of $\left.\mathrm{M}_{\mathrm{GSp}, S^{ \pm}}^{\mathrm{loc}}\right)$ with the closure of $G \cdot \mu \subset \mathrm{GL}\left(V_{\mathbb{Z}_{p}}\right) / P_{h^{-1}}$.

4.1.6. Let $V_{\mathbb{Z}_{(p)}}=V_{\mathbb{Z}_{p}} \cap V_{\mathbb{Q}}$, and fix a $\mathbb{Z}$-lattice $V_{\mathbb{Z}} \subset V_{\mathbb{Q}}$ such that $V_{\mathbb{Z}} \otimes_{\mathbb{Z}} \mathbb{Z}_{(p)}=$ $V_{\mathbb{Z}_{(p)}}$ and $V_{\mathbb{Z}} \subset V_{\mathbb{Z}}^{\vee}$. The choice of lattice $V_{\mathbb{Z}}$ gives rise to an interpretation of $\mathrm{Sh}_{\mathrm{K}^{\prime}}\left(\mathrm{GSp}, S^{ \pm}\right)$as a moduli space of polarized abelian varieties. 
Consider the Zariski closure $G_{\mathbb{Z}_{(p)}}$ of $G$ in $\operatorname{GL}\left(V_{\mathbb{Z}_{(p)}}\right)$; then $G_{\mathbb{Z}_{(p)}} \otimes_{\mathbb{Z}_{(p)}} \mathbb{Z}_{p} \cong \mathcal{G}$. Set $\mathrm{K}_{p}=\mathcal{G}\left(\mathbb{Z}_{p}\right)$, and $\mathrm{K}_{p}^{\prime}=\mathcal{G S P}\left(\mathbb{Z}_{p}\right)$. We set $\mathrm{K}=\mathrm{K}_{p} \mathrm{~K}^{p}$ and similarly for $\mathrm{K}^{\prime}$. By [43] Lemma 2.1.2, for any compact open subgroup $\mathrm{K}^{p} \subset G\left(\mathbb{A}_{f}^{p}\right)$ there exists $\mathrm{K}^{\prime p} \subset \mathrm{GSp}\left(\mathbb{A}_{f}^{p}\right)$ such that $\iota$ induces an embedding over $\mathrm{E}$

$$
\operatorname{Sh}_{\mathrm{K}}(G, X) \hookrightarrow \mathrm{Sh}_{\mathcal{K}^{\prime}}\left(\mathrm{GSp}, S^{ \pm}\right) .
$$

4.1.7. We now introduce Hodge cycles. Fix a collection of tensors $\left(s_{\alpha}\right) \subset V_{\mathbb{Z}_{(p)}}^{\otimes}$ whose stabilizer is $G_{\mathbb{Z}_{(p)}}$. This is possible by [43] Lemma 1.3.2.

Let $h: \mathcal{A} \rightarrow \operatorname{Sh}_{K}(G, X)$ denote the restriction to $\operatorname{Sh}_{K}(G, X)$ of the universal abelian scheme, and let $\mathcal{V}=R^{1} h_{*} \Omega^{\bullet}$ be the de Rham cohomology of $\mathcal{A}$. As in 43] $\S 2.2$, the $s_{\alpha}$ give rise to a collection of absolute Hodge cycles $s_{\alpha, \mathrm{dR}} \in \mathcal{V}^{\otimes}$, defined over the reflex field $\mathrm{E}$.

Now let $\kappa \supset \mathrm{E}$ be a field of characteristic 0 , and $\bar{\kappa}$ an algebraic closure of $\kappa$. Fix an embedding $\mathbb{Q}_{p} \hookrightarrow \mathbb{C}$ and an embedding of $E$-algebras $\sigma: \bar{\kappa} \hookrightarrow \mathbb{C}$. Let $x \in \operatorname{Sh}_{K}(G, X)(\kappa)$ and denote by $\mathcal{A}_{x}$ the corresponding abelian variety over $\kappa$. Denote by $H_{B}^{1}\left(\mathcal{A}_{x}(\mathbb{C}), \mathbb{Q}\right)$ the Betti cohomology of $\mathcal{A}_{x}(\mathbb{C})$. Write $H_{\mathrm{dR}}^{1}\left(\mathcal{A}_{x}\right)$ for its de Rham cohomology and $H_{\text {êt }}^{1}\left(\mathcal{A}_{x, \bar{\kappa}}\right)=H_{\text {ét }}^{1}\left(\mathcal{A}_{x, \bar{\kappa}}, \mathbb{Q}_{p}\right)$ for the $p$-adic étale cohomology of $\mathcal{A}_{x, \bar{\kappa}}=\mathcal{A}_{x} \otimes_{\kappa} \bar{\kappa}$. The embedding $\sigma$ induces isomorphisms

$$
H_{\mathrm{dR}}^{1}\left(\mathcal{A}_{x}\right) \otimes_{\kappa, \sigma} \mathbb{C} \cong H_{B}^{1}\left(\mathcal{A}_{x}(\mathbb{C}), \mathbb{Q}\right) \otimes_{\mathbb{Q}} \mathbb{C} \cong H^{1}\left(\mathcal{A}_{x, \bar{\kappa}}, \mathbb{Q}_{p}\right) \otimes_{\mathbb{Q}_{p}} \mathbb{C} .
$$

Let $s_{\alpha, \mathrm{dR}, x}$ denote the fibre of $s_{\alpha, \mathrm{dR}}$ over $x$, and $s_{\alpha, \text { ét }, x} \in H_{\text {ét }}^{1}\left(\mathcal{A}_{x, \bar{\kappa}}\right)^{\otimes}$ the image of $s_{\alpha, \mathrm{dR}, x}$ under the composite of the above two isomorphisms. As in 43 Lemma 2.2.1 one sees that $s_{\alpha \text {,ét, } x}$ is $\operatorname{Gal}(\bar{\kappa} / \kappa)$-invariant and, in particular, independent of the choices made above.

\subsection{Integral models.}

4.2.1. We keep the notation and assumptions introduced above.

Fix a prime $v \mid p$ of $\mathrm{E}$, and let $\mathcal{O}$ be the ring of integers of $\mathrm{E}$, and $k_{v}$ the residue field of $v$. The choice of lattice $V_{\mathbb{Z}}$ gives rise to an interpretation of $\operatorname{Sh}_{\mathrm{K}^{\prime}}\left(\mathrm{GSp}, S^{ \pm}\right)$as a moduli space of polarized abelian varieties, and hence to a natural integral model $\mathscr{S}_{\mathrm{K}^{\prime}}\left(\mathrm{GSp}, S^{ \pm}\right)$over $\mathbb{Z}_{p}$, and hence over $\mathcal{O}_{(v)}$. We denote by $\mathscr{S}_{\mathrm{K}}^{-}(G, X)$ the closure of $\mathrm{Sh}_{\mathrm{K}}(G, X)$ in the $\mathcal{O}_{(v)}$-scheme $\mathscr{S}_{\mathrm{K}^{\prime}}\left(\mathrm{GSp}, S^{ \pm}\right)$, and by $\mathscr{S}_{\mathrm{K}}(G, X)$, the normalization of $\mathscr{S}_{\mathrm{K}}(G, X)^{-}$.

Fix an algebraic closure $\overline{\mathbb{Q}}_{p}$ of $\mathbb{Q}_{p}$, and an embedding $v: \mathrm{E} \hookrightarrow \overline{\mathbb{Q}}_{p}$. Let $E=\mathrm{E}_{v}$, so that $E$ is the local reflex field of $\left(G,\left\{\mu_{h}\right\}\right)$. We denote by $k$ the residue field of $\overline{\mathbb{Q}}_{p}$ and write $W=W(k)$ and $K_{0}=W[1 / p]$. Set $E^{\mathrm{ur}}=E \cdot K_{0}$ in the completion of $\overline{\mathbb{Q}}_{p}$.

Let $K / E^{\text {ur }}$ be a finite extension, and let $x \in \operatorname{Sh}_{K}(G, X)(K)$ be a point which admits a specialization $\bar{x} \in \mathscr{S}_{\mathrm{K}}^{-}(G, X)(k)$. Let $\mathscr{G}_{x}$ denote the $p$-divisible group over the $\mathcal{O}_{K}$-valued point corresponding to $x$, and $\mathscr{G}_{\bar{x}}$ its special fibre. Write $\mathbb{D}_{\bar{x}}=$ $\mathbb{D}\left(\mathscr{G}_{\bar{x}}\right)(W)$. Let $s_{\alpha, 0} \in \mathbb{D}_{\bar{x}}^{\otimes} \otimes_{\mathbb{Z}_{p}} \mathbb{Q}_{p}$ be the $\varphi$-invariant tensors corresponding to $s_{\alpha, \text { ét, } x}$ under the $p$-adic comparison isomorphism, and $G_{K_{0}} \subset \mathrm{GL}\left(\mathbb{D}_{\bar{x}} \otimes_{\mathbb{Z}_{p}} \mathbb{Q}_{p}\right)$ the group defined by $\left(s_{\alpha, 0}\right)$. The filtration on $\mathbb{D}_{K}$ corresponding to $\mathscr{G}_{x}$ corresponds to a parabolic in $G_{K_{0}} \otimes_{K_{0}} K$ by [4] Lemma 1.4 .5 (in the terminology of loc. cit., this filtration is $G$-split). This is induced by a $G$-valued cocharacter which lies in the $G$-conjugacy class of $\mu_{h}^{-1}$.

We use the notation of 3.3 .11 applied with $\mathscr{G}=\mathscr{G}_{x}$. Thus we have a parabolic subgroup $P \subset \mathrm{GL}\left(\mathbb{D}_{\bar{x}}\right)$, and a point $y=y(x) \in\left(\mathrm{GL}\left(\mathbb{D}_{\bar{x}}\right) / P\right)(K)$, which specializes 
to $P_{0}=P \otimes_{W} k$, and is induced by a $G$-valued cocharacter $\mu_{y}$, which is conjugate to $\mu_{h^{-1}}$. We obtain formal local models $\widehat{\mathrm{M}}_{y}^{\text {loc }}=\operatorname{Spf} R$ and $\widehat{\mathrm{M}}_{G, y}^{\text {loc }}=\operatorname{Spf} R_{G}$ defined over $\mathcal{O}_{E}$, the latter being obtained by completing the orbit closure $\mathrm{M}_{G}^{\text {loc }}:=\overline{G_{K_{0}} \cdot y(x)} \subset$ $\operatorname{GL}\left(\mathbb{D}_{\bar{x}}\right) / P$ at the specialization of $y$.

Proposition 4.2.2. Let $\widehat{U}_{\bar{x}}$ be the completion of $\mathscr{S}_{\mathrm{K}}^{-}(G, X)_{\mathcal{O}_{E} \mathrm{ur}}$ at $\bar{x}$. Then the irreducible component of $\widehat{U}_{\bar{x}}$ containing $x$ is isomorphic to $\widehat{\mathrm{M}}_{G, y}^{\text {loc }}$ as formal schemes over $\mathcal{O}_{E \text { ur }}$.

Proof. Recall that we are assuming that $G$ splits over a tamely ramified extension, and that $\mathcal{G}^{\circ}$ is a parahoric group scheme. Note that $G_{K_{0}} \subset \mathrm{GL}\left(\mathbb{D}_{\bar{x}} \otimes_{\mathbb{Z}_{p}} \mathbb{Q}_{p}\right)$ contains the scalars, since $G \subset \mathrm{GL}\left(V_{\mathbb{Q}}\right)$ contains the image of $w_{h}$, and $R_{G}$ is normal by Theorem 2.1.2. It follows that the conditions imposed in the construction of (3.3.11) are satisfied, and we can equip $\mathbb{D} \otimes_{W} R$ with the structure of a Dieudonné display over $R$ satisfying the conditions in 3.2 .12

In particular, this construction allows us to view $R$ as a versal deformation ring for $\mathscr{G}_{\bar{x}}$, so there exists a map $\Theta: \widehat{U}_{\bar{x}} \rightarrow \widehat{M}_{y}^{\text {loc }}$ such that the $p$-divisible group corresponding to the chosen Dieudonné display over $R$ pulls back to the $p$-divisible group over $\widehat{U}_{\bar{x}}$ arising from the universal family of abelian schemes over $\widehat{U}_{\bar{x}}$. By the Serre-Tate theorem, $\Theta$ is a closed embedding, and it suffices to show that it factors through $\widehat{\mathrm{M}}_{G, y}^{\text {loc }}$ since both $\widehat{\mathrm{M}}_{G, y}^{\mathrm{loc}}$ and $\widehat{U}_{\bar{x}}$ have the same dimension.

Let $K^{\prime} \supset E^{\text {ur }}$ be any finite extension and $x^{\prime} \in \widehat{U}_{\bar{x}}\left(K^{\prime}\right)$ a point lying on the same irreducible component of $\widehat{U}_{\bar{x}}$ as $x$. The same argument as in 43. Proposition 2.3.5 shows that $s_{\alpha, \text { ét, } x^{\prime}}$ corresponds to $s_{\alpha, 0}$ under the $p$-adic comparison isomorphism for the $p$-divisible group $\mathscr{G}_{x^{\prime}}$ : Let $\mathcal{U}$ and $\widehat{U}_{\bar{x}}^{\text {an }}$ denote the analytic spaces over $E^{\text {ur }}$ attached to $\operatorname{Spf} R$ and $\widehat{U}_{\bar{x}}$ respectively [19, $\S 7$. Since $\mathbb{D}_{R}=\mathbb{D} \otimes_{W} R$ underlies an $F$-isocrystal on $R$, the sections $s_{\alpha, 0}$ extend uniquely to parallel sections $\tilde{s}_{\alpha, 0} \in \mathbb{D}_{R}^{\otimes} \mid \mathcal{U}$ [42, 3.1]. The isomorphism $H_{\text {cris }}^{1}\left(\mathcal{A}_{\bar{x}} / W\right) \otimes K^{\prime} \cong H_{\mathrm{dR}}^{1}\left(\mathcal{A}_{x^{\prime}}\right)$ takes $s_{\alpha, 0}$ to $\left.\tilde{s}_{\alpha, 0}\right|_{x^{\prime}}$, 4 , $\S 2.9]$. Now $\left.\left(\tilde{s}_{\alpha, 0}-s_{\alpha, d R}\right)\right|_{\widehat{U}_{\bar{x}}^{\text {an }}}$ is a parallel section of $\left.\mathbb{D}_{R}^{\otimes}\right|_{\widehat{U}_{\bar{x}}^{\text {an }}}$ which vanishes at $x$ by construction. Hence it vanishes on the irreducible component of $\widehat{U}_{\bar{x}}^{\text {an }}$ containing $x$, and in particular at $x^{\prime}$, so that $\left.\tilde{s}_{\alpha, 0}\right|_{x^{\prime}}=s_{\alpha, \mathrm{dR}, x^{\prime}}$. Finally $s_{\alpha, d R, x^{\prime}}$ and $s_{\alpha \text {,ét, } x^{\prime}}$ correspond under the $p$-adic comparison isomorphism [5].

Since the filtration on $\mathbb{D}_{\bar{x}} \otimes_{K_{0}} K^{\prime}$ corresponding to $\mathscr{G}_{x^{\prime}}$ is given by a cocharacter which is conjugate to $\mu_{h}^{-1}$. It follows from Lemma 3.3 .13 that $x^{\prime}$ is induced by a point of $\widehat{M}_{G, y}^{\text {loc }}$. Since this holds for any $x^{\prime}$, it follows that $\Theta$ factors through $\widehat{M}_{G, y}^{\text {loc }}$.

4.2.3. Let $k_{E}$ denote the residue field of $E$. If $k^{\prime} / k_{E}$ is an extension of perfect fields, and $z \in \mathrm{M}_{G, X}^{\mathrm{loc}}\left(k^{\prime}\right)$, we denote by $\mathrm{M}_{G, X}^{\mathrm{loc}} \otimes_{W\left(k_{E}\right)} W\left(k^{\prime}\right)$ by $\mathrm{M}_{G, X, z}^{\mathrm{loc}}$, and by $\widehat{\mathrm{M}}_{G, X, z}^{\mathrm{loc}}$, the completion of $\mathrm{M}_{G, X, z}^{\mathrm{loc}}$, at the image of $z$.

Corollary 4.2.4. Let $\bar{x} \in \mathscr{S}_{\mathrm{K}}(G, X)$ be a closed point of characteristic $p$. Then there exists $z \in \mathrm{M}_{G, X}^{\mathrm{loc}}(k)$ such that $\widehat{U}_{x}$ is isomorphic to $\widehat{\mathrm{M}}_{G, X, z}^{\mathrm{loc}}$ over $\mathcal{O}_{E^{\text {ur }}}$.

Proof. By Proposition 3.3.8 we may identify the subgroup $\mathcal{G}_{W} \subset \mathrm{GL}(\mathbb{D})$ with the pullback to $\mathcal{O}_{E^{\text {ur }}}$ of $\mathcal{G} \subset \mathrm{GL}\left(V_{\mathbb{Z}_{p}}\right)$. Hence the lemma follows from Proposition 4.2.2 The fact that $y$ corresponds to a parabolic of $G$ was already remarked above.

4.2.5. We continue to assume that $G$ splits over a tamely ramified extension of $\mathbb{Q}_{p}$ and that $p$ does not divide the order of $\pi_{1}\left(G^{\text {der }}\right)$. The relationship between 
the integral model $\mathscr{S}_{\mathrm{K}}(G, X)$ and local models can be globalized. To explain this, recall that we have the bundle $\mathcal{V}=R^{1} h_{*} \Omega^{\bullet}$ over $\operatorname{Sh}_{K}(G, X)$ given by first de Rham cohomology of the universal abelian scheme and a collection of absolute Hodge cycles $s_{\alpha, \mathrm{dR}} \in \mathcal{V}^{\otimes}$, all defined over the reflex field $\mathrm{E}$. The bundle $\mathcal{V}$ extends to a bundle $\underline{\mathcal{V}}$ over the $\mathcal{O}_{\mathrm{E}_{(v)}}$-scheme $\mathscr{S}_{\mathrm{K}}(G, X)$.

Consider now the $G$-torsor $\widetilde{\operatorname{Sh}_{K}}(G, X)$ over $\operatorname{Sh}_{K}(G, X)$ classifying trivializations $f: V^{\vee} \stackrel{\sim}{\rightarrow} \mathcal{V}$ that preserve the tensors, i.e. with $f^{\otimes}\left(s_{\alpha}\right)=s_{\alpha, \mathrm{dR}}$.

Proposition 4.2.6. The $s_{\alpha, \mathrm{dR}}$ extend to tensors $s_{\alpha, \mathrm{dR}} \in \underline{\mathcal{V}}^{\otimes}$ over $\mathscr{S}_{\mathrm{K}}(G, X)$. The scheme $\widetilde{\mathscr{S}_{\mathrm{K}}}(G, X)$ that classifies trivializations $f: V_{\mathbb{Z}_{(p)}}^{\vee} \stackrel{\sim}{\rightarrow} \underline{\mathcal{V}}$ with $f^{\otimes}\left(s_{\alpha}\right)=\underline{s}_{\alpha, \mathrm{dR}}$, is a $\mathcal{G}$-torsor over $\mathscr{S}_{\mathrm{K}}(G, X)$.

Proof. As in the proof of Corollary 3.2.11 since $\mathscr{S}_{k}(G, X)$ is normal, to show that $s_{\alpha, \mathrm{dR}}$ belongs to $\underline{\mathcal{V}}^{\otimes}$, it is enough to check that for every $\xi: \operatorname{Spec}\left(\mathcal{O}_{K}\right) \rightarrow \mathscr{S}_{\mathrm{K}}(G, X)$ with $K$ a finite extension of $E, s_{\alpha, \mathrm{dR}, \xi}$ is in $\xi^{*}(\underline{\mathcal{V}})$ (where we again denote by $\xi$ the $K$-valued point corresponding to $\xi$ ). A result of Blasius and Wintenberger $[5$ asserts that the $p$-adic comparison isomorphism takes $s_{\alpha, \text { ét, } \xi}$ to $s_{\alpha, \mathrm{dR}, \xi}$. Let $\mathscr{G}_{\xi}$ denote the pullback via $\xi$ of the universal $p$-divisible group over $\mathscr{S}_{\mathfrak{K}}(G, X)$. If $\mathfrak{M}=\mathfrak{M}\left(T_{p} \mathscr{G}_{\xi}^{\vee}\right)$ then by Theorem 3.3.2, we have

$$
s_{\alpha, \mathrm{dR}, \xi} \in \mathcal{O}_{K} \otimes_{\mathfrak{S}} \varphi^{*}(\mathfrak{M})^{\otimes} \cong \mathbb{D}\left(\mathscr{G}_{\xi}\right)\left(\mathcal{O}_{K}\right)^{\otimes} \cong \xi^{*}(\underline{\mathcal{V}})^{\otimes} .
$$

It follows by Proposition 3.3 .8 that $\xi^{*}\left(\widetilde{\mathscr{S}}_{\mathrm{K}}(G, X)\right)$ is a $\mathcal{G}$-torsor. Arguing as in the proof of Corollary 3.2 .11 we see that $\widetilde{\mathscr{S}_{K}}(G, X)$ is a $\mathcal{G}$-torsor.

Theorem 4.2.7. Under the above assumptions, there exists a diagram of morphisms

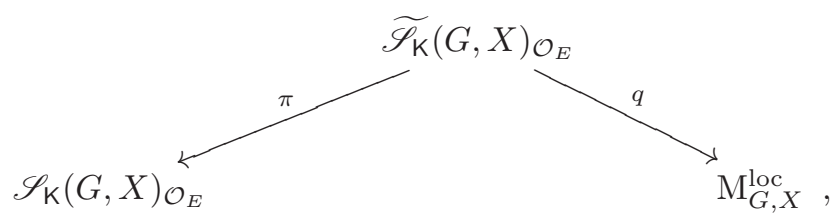

of $\mathcal{O}_{E}$-schemes, in which:

- $\pi$ is the $\mathcal{G}$-torsor given by Proposition 4.2.6.

- $q$ is $\mathcal{G}$-equivariant and smooth of relative dimension $\operatorname{dim} G$.

Proof. Let $K / E^{\mathrm{ur}}$ be a finite extension, and $x \in \operatorname{Sh}_{K}(G, X)(K)$ be a point which admits a specialization $\bar{x} \in \mathscr{S}_{\mathrm{K}}^{-}(G, X)(k)$. We use the notation introduced in 4.2.1. In particular, we have the orbit closure $\mathrm{M}_{G}^{\text {loc }}:=\overline{G_{K_{0}} \cdot y(x)} \subset \mathrm{GL}\left(\mathbb{D}_{\bar{x}}\right) / P$. By Proposition 3.3.4 and Lemma 3.3.8 we have $s_{\alpha, 0, \bar{x}} \in \mathbb{D}_{\bar{x}}^{\otimes}$ and if $\widetilde{\mathrm{M}}_{G}^{\text {loc }}$ is the scheme over $\mathrm{M}_{G}^{\text {loc }}$ which parametrizes isomorphisms $f: \mathbb{D}_{\bar{x}} \cong V_{\mathbb{Z}_{p}}^{\vee} \otimes_{\mathbb{Z}_{p}} W$ such that $f^{\otimes}\left(s_{\alpha, 0, \bar{x}}\right)=s_{\alpha}$, then $\widetilde{\mathrm{M}}_{G}^{\text {loc }}=\mathcal{P} \times \mathrm{M}_{G}^{\text {loc }}$, where $\mathcal{P}$ is a trivial $\mathcal{G}_{W}$-torsor. In particular $\widetilde{\mathrm{M}}_{G}^{\text {loc }}$ is a $\mathcal{G}$ -

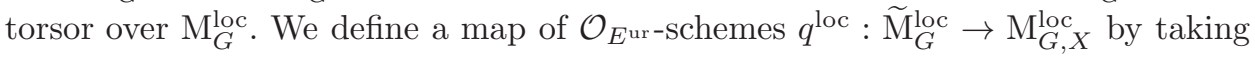

\footnotetext{
${ }^{6}$ Indeed this result was already used implicitly via the citation of 43 in the proof of Lemma 4.2 .2 above.
} 
$(f, \mathcal{F})$ to $f^{-1}(\mathcal{F})$. One sees easily that $q^{\text {loc }}$ is a $\mathcal{G}$-torsor. Thus we have a diagram

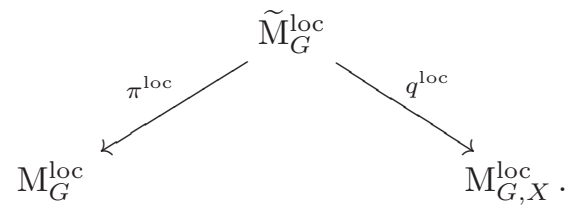

with $\pi^{\text {loc }}$ and $q^{\text {loc }}$ are $\mathcal{G}$-torsors.

To construct the morphism $q$, let $(x, f)$ be an $S$-valued point of $\widetilde{\mathscr{S}_{K}}(G, X)$. We send $(x, f)$ to the inverse image $f^{-1}(\mathcal{F}) \subset V_{\mathbb{Z}_{p}}^{\vee} \otimes \mathcal{O}_{S}$ of the Hodge filtration $\mathcal{F} \subset \underline{\mathcal{V}}(S)=R^{1} h_{*} \Omega_{A / S}^{\bullet}$, which is an $S$-valued point of $\operatorname{GL}\left(V_{\mathbb{Z}_{p}}\right) / P_{h}$. Now consider the diagram of $\mathcal{O}_{E}$-schemes

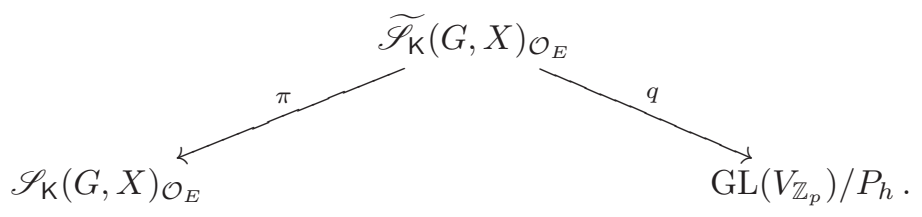

We have to show that $q$ factors through $\mathrm{M}_{G, X}^{\text {loc }}$ and that the resulting morphism to $\mathrm{M}_{G, X}^{\text {loc }}$ is smooth of relative dimension $\operatorname{dim} G$. To do this it suffices to show these properties for the corresponding morphism in the diagram of $\mathcal{O}_{E}$ ur-schemes

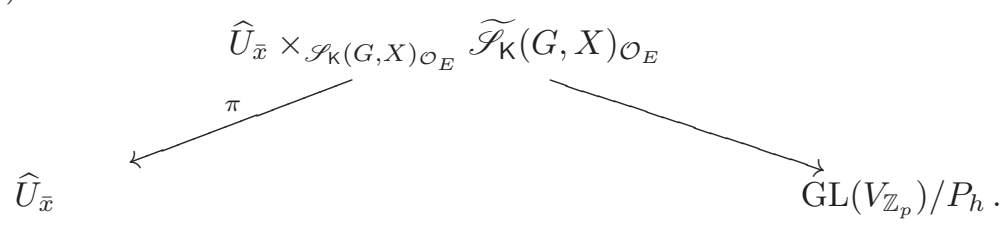

Here we have written $\widehat{U}_{\bar{x}}$ for the affine scheme with the same affine ring as the formal scheme $\widehat{U}_{\bar{x}}$, and the map on the left of the diagram is obtained by pulling back $\pi$ by $\widehat{U}_{\bar{x}} \rightarrow \mathscr{S}_{K}(G, X)_{\mathcal{O}_{E}}$. One sees directly from the definitions that this last diagram can be identified with the one obtained from 4.2.9 by pulling back $\pi^{\text {loc }}$ by the isomorphism $\widehat{U}_{\bar{x}} \cong \widehat{\mathrm{M}}_{G}^{\text {loc }} \rightarrow \mathrm{M}_{G}^{\text {loc }}$, given by Proposition 4.2.2. Since $q^{\text {loc }}$ has the required properties, so does $q$.

Corollary 4.2.12. Under the above assumptions, the scheme $\mathscr{S}_{\mathrm{K}}(G, X)$ has reduced special fibre. If $\mathrm{K}_{p}=\mathrm{K}_{p}^{\circ}$, i.e. $\mathrm{K}_{p}$ is parahoric, then the geometric special fibre $\mathscr{S}_{K}(G, X) \otimes_{\mathcal{O}_{E}} k$ admits a stratification with locally closed strata parametrized by the $\mu$-admissible set of Kottwitz and Rapoport (e.g. [59, 9.1.2); the closure of each stratum is normal and Cohen-Macaulay.

Proof. This follows from the existence of the diagram (4.2.8) and [59] Theorem 1.1 by the standard argument (see loc. cit. Theorem 1.2.) Indeed, the stratification is obtained from a $\mathcal{G}$-stratification on the geometric special fibre $\mathrm{M}_{G, X}^{\mathrm{loc}} \otimes_{\mathcal{O}_{E}} k$ which is given by realizing this as a union of affine Schubert varieties in an affine Grassmannian.

Corollary 4.2.13. Under the above assumptions, including $\mathrm{K}_{p}=\mathrm{K}_{p}^{\circ}$, given a point $z \in \mathscr{S}_{\mathrm{K}}(G, X)\left(\mathbb{F}_{q}\right), k_{E} \subset \mathbb{F}_{q}$, there is $w \in \mathrm{M}_{G, X}^{\mathrm{loc}}\left(\mathbb{F}_{q}\right)$, well defined up to the action 
of $\mathcal{G}\left(\mathbb{F}_{q}\right)$ on $\mathrm{M}_{G, X}^{\mathrm{loc}}\left(\mathbb{F}_{q}\right)$, such that we have an isomorphism of henselizations

$$
\mathcal{O}_{\mathscr{S}_{K}(G, X), z}^{\mathrm{h}} \simeq \mathcal{O}_{\mathrm{M}_{G, X}^{\mathrm{loc}, w}}^{\mathrm{h}} \text {. }
$$

Proof. For simplicity, set $\mathscr{S}=\mathscr{S}_{\mathrm{K}}(G, X)$. Lang's lemma applied to the torsor $\pi_{\mathbb{F}_{q}}$ for the smooth connected group scheme $\mathcal{G}_{\mathbb{F}_{q}}$ implies that there is $\tilde{z} \in \widetilde{\mathscr{S}}\left(\mathbb{F}_{q}\right)$ that lifts $z$, i.e. $\pi(\tilde{z})=z$, and we take $w=q(\tilde{z})$. Since both $\pi$ and $q$ are smooth, we there is a section $\operatorname{Spec}\left(\mathcal{O}_{\mathscr{S}, z}^{\mathrm{h}}\right) \rightarrow \widetilde{\mathscr{S}}$ which extends $\tilde{z}$ and is such that the composition $b: \operatorname{Spec}\left(\mathcal{O}_{\mathscr{S}, z}^{\mathrm{h}}\right) \rightarrow \widetilde{\mathscr{S}} \rightarrow \mathrm{M}_{G, X}^{\mathrm{loc}}$ induces an injection of tangent spaces at $z$ and $w$. This injection is necessarily an isomorphism and so $b$ is formally étale. The result follows.

Remark 4.2.14. a) We expect that the map $q: \widetilde{\mathscr{S}_{\mathrm{K}}}(G, X) \rightarrow \mathrm{M}_{G, X}^{\mathrm{loc}}$ is surjective. However, we don't know how to uniquely characterize the model $\mathscr{S}_{\mathrm{K}}(G, X)$ of $\operatorname{Sh}_{\mathrm{K}}(G, X)$ in general, even after assuming this statement. However, see 4.6 .27 for a partial result in this direction.

b) Assume that $G$ is unramified over $\mathbb{Q}_{p}$, i.e. quasi-split over $\mathbb{Q}_{p}$ and split over an unramified extension of $\mathbb{Q}_{p}$. Then given $x \in \mathcal{B}\left(G, \mathbb{Q}_{p}\right)$, such that $\mathcal{G}_{x}^{\circ}\left(\mathbb{Z}_{p}\right)$ is contained in a hyperspecial subgroup, there is $x^{\prime} \in \mathcal{B}\left(G, \mathbb{Q}_{p}\right)$ such that $\mathcal{G}_{x}^{\circ}=\mathcal{G}_{x^{\prime}}$. (One can take $x^{\prime}$ to be a generic point of the smallest facet that contains $x$, see e.g. Lemma 7.0.2 of the Corrigendum for [35.) Therefore, in this case, Theorem 4.2 .7 and its corollaries can be applied to Shimura varieties for all such parahoric subgroups of $G\left(\mathbb{Q}_{p}\right)$.

\subsection{Integral models for parahoric level.}

4.3.1. We will use the results of the previous section to construct integral models for Shimura varieties of Hodge type with parahoric level structure. That is, where the level structure at $p$ is given by $\mathcal{G}^{\circ}\left(\mathbb{Z}_{p}\right)$. We keep the notation introduced above, and write $\mathrm{K}_{p}^{\circ}=\mathcal{G}^{\circ}\left(\mathbb{Z}_{p}\right)$ and $\mathrm{K}^{\circ}=\mathrm{K}_{p}^{\circ} \mathrm{K}^{p}$. We denote by $\tilde{G}$ the universal cover of $G^{\text {der }}$. We begin with two lemmas.

Lemma 4.3.2. The composite of the maps

$$
E^{\times} \stackrel{\left[\mu_{h}^{-1}\right]}{\rightarrow} G(E) / \tilde{G}(E) \rightarrow G\left(\mathbb{Q}_{p}\right) / \tilde{G}\left(\mathbb{Q}_{p}\right) \rightarrow G\left(\mathbb{Q}_{p}\right) / \tilde{G}\left(\mathbb{Q}_{p}\right) \mathrm{K}_{p}^{\circ}
$$

is trivial on $\mathcal{O}_{E}^{\times}$. Here the first map is induced by the conjugacy class of $\mu_{h}^{-1}$ for $h \in X$, and the second map is given by the norm $N_{E / \mathbb{Q}_{p}}$.

Proof. Let $E_{0} \subset E$ be the maximal unramified subfield of $E$, and set $\mathrm{K}_{p, E_{0}}^{\circ}=$ $\mathcal{G}_{x}^{\circ}\left(\mathcal{O}_{E_{0}}\right)$. It suffices to show that the composite

$$
E^{\times} \stackrel{\left[\mu_{h}^{-1}\right]}{\rightarrow} G(E) / \tilde{G}(E) \stackrel{N_{E / E_{0}}}{\rightarrow} G\left(E_{0}\right) / \tilde{G}\left(E_{0}\right) \mathrm{K}_{p, E_{0}}^{\circ}
$$

kills $\mathcal{O}_{E}^{\times}$, since then the lemma follows by applying $N_{E_{0} / \mathbb{Q}_{p}}$.

To show this, we may replace $E$ by $E \cdot E_{0}^{\prime}$, where $E_{0}^{\prime}$ is a finite unramified extension of $E_{0}$, and assume that $G$ is quasi-split over $E_{0}$. Let $T$ be the centralizer of a maximal split torus in $G_{E_{0}}$. Then $\left[\mu_{h}^{-1}\right]$ contains a cocharacter $\mu \in \mathbb{X}_{\bullet}(T)$, defined over $E$. After replacing $\mathrm{K}_{p, E_{0}}^{\circ}$ by a conjugate subgroup, we may assume that the point $x \in \mathcal{B}\left(G, E_{0}\right)$ defining $\mathcal{G}^{\circ}$ is in the apartment corresponding to $T$.

Let $\mathcal{T}^{\circ}$ denote the connected Néron model of $T$. Consider the composite

$$
R_{E / E_{0}} \mathbb{G}_{m} \stackrel{\mu}{\rightarrow} R_{E / E_{0}} T \stackrel{N_{E / E_{0}}}{\rightarrow} T .
$$


The corresponding map on $E_{0}$-points sends $\mathcal{O}_{E}^{\times}$to a bounded subgroup of $T\left(E_{0}\right)$. If $\Gamma_{E_{0}}=\operatorname{Gal}\left(\overline{\mathbb{Q}}_{p} / E_{0}\right)$, then $\pi_{1}\left(R_{E / E_{0}} \mathbb{G}_{m}\right)_{\Gamma_{E_{0}}}=\mathbb{Z}$ is torsion free, and in particular, the image of $\mathcal{O}_{E}^{\times}$in $\pi_{1}\left(R_{E / E_{0}} \mathbb{G}_{m}\right)_{\Gamma_{E_{0}}}$ and $\pi_{1}(T)_{\Gamma_{E_{0}}}$ is trivial. Hence the above map sends $\mathcal{O}_{E}^{\times}$into $\mathcal{T}^{\circ}\left(\mathcal{O}_{E_{0}}\right)$. Since $\mathcal{T}^{\circ}\left(\mathcal{O}_{E_{0}}\right) \subset \mathrm{K}_{p, E_{0}}^{\circ}$, the lemma follows.

4.3.3. Now let $C=\operatorname{ker}\left(\tilde{G} \rightarrow G^{\text {der }}\right)$. For $c \in H^{1}(\mathbb{Q}, C)$, and $l$ a finite prime, denote by $c_{l} \in H^{1}\left(\mathbb{Q}_{l}, C\right)$ the image of $c$. Until further notice, we assume that $G$ satisfies the following condition.

$$
\text { If } c \in H^{1}(\mathbb{Q}, C) \text { satisfies } c_{l}=0 \text { for } l \neq p \text {, then } c_{p}=0 .
$$

The condition will be removed at the end, and so does not appear in our final result.

Lemma 4.3.5. For $\mathrm{K}^{p}$ sufficiently small

$$
G(\mathbb{Q}) \cap \mathrm{K} \subset \mathrm{K}^{\circ} .
$$

Proof. Let $\rho: \tilde{G} \rightarrow G$ denote the natural map. By [20, Cor. 2.0.5, 2.0.13], we may choose $\mathrm{K}^{p}$ sufficiently small that

$$
\mathrm{K}^{p} \cap G(\mathbb{Q}) \subset\left(\rho \tilde{G}\left(\mathbb{A}_{f}^{p}\right) \cap G(\mathbb{Q})\right) \cdot U \subset G\left(\mathbb{A}_{f}^{p}\right),
$$

where $U$ is any subgroup of finite index in the $p$-units in $Z_{G}(\mathbb{Q})$.

By [20, Prop. 2.0.4(ii)], our assumption that (4.3.4) holds implies that

$$
\rho \tilde{G}\left(\mathbb{A}_{f}^{p}\right) \cap G(\mathbb{Q})=\rho \tilde{G}\left(\mathbb{A}_{f}\right) \cap G(\mathbb{Q}) .
$$

Here the intersection on the left (resp. right) is taken in $G\left(\mathbb{A}_{f}^{p}\right)$ (resp. $G\left(\mathbb{A}_{f}\right)$ ). Thus $\mathrm{K} \cap G(\mathbb{Q}) \subset \rho \tilde{G}\left(\mathbb{A}_{f}\right) \cdot U$. In particular for $U$ and $\mathrm{K}^{p}$ sufficiently small $\mathrm{K} \cap G(\mathbb{Q}) \subset \mathrm{K}^{\circ}$ by [38, Prop. 3.

4.3.6. Let $G(\mathbb{Q})_{+}$denote the preimage of $G^{\text {ad }}(\mathbb{R})^{+}$in $G(\mathbb{Q})$, and let $G(\mathbb{Q})_{+}^{-}$be the closure of $G(\mathbb{Q})_{+}$in $G\left(\mathbb{A}_{f}\right)$. Denote by $\mathscr{S}_{K^{\circ}}(G, X)$ the normalization of $\mathscr{S}_{\mathfrak{K}}(G, X)$ in $\operatorname{Sh}_{K^{\circ}}(G, X)$.

Proposition 4.3.7. If $\mathrm{K}^{p}$ satisfies the smallness assumption imposed in 4.1.1) then the covering $\mathscr{S}_{\mathrm{K}^{\circ}}(G, X) \rightarrow \mathscr{S}_{\mathrm{K}}(G, X)$ is étale. If $\mathrm{K}^{p}$ is sufficiently small, this covering splits over an unramified extension of $\mathcal{O}_{E}$.

Proof. By [20] 2.1.3.1, the connected components of $\operatorname{Sh}_{K^{\circ}}(G, X)\left(\operatorname{resp} . \operatorname{Sh}_{\mathcal{K}}(G, X)\right)$ over $\overline{\mathbb{Q}}_{p}$ form a torsor under $G\left(\mathbb{A}_{f}\right) / G(\mathbb{Q})_{+} \mathrm{K}^{\circ}$ (resp. $\left.G\left(\mathbb{A}_{f}\right) / G(\mathbb{Q})_{+} \mathrm{K}\right)$, which is an abelian group. Suppose that $\mathrm{K}^{p}$ is sufficiently small, so that the conclusion of Lemma 4.3.5 holds. Then

$$
\pi_{0}\left(\operatorname{Sh}_{K^{\circ}}(G, X)_{\overline{\mathbb{Q}}_{p}}\right) \rightarrow \pi_{0}\left(\operatorname{Sh}_{\mathcal{K}}(G, X)_{\overline{\mathbb{Q}}_{p}}\right)
$$

is a torsor under

$G(\mathbb{Q})_{+} \mathrm{K} / G(\mathbb{Q})_{+} \mathrm{K}^{\circ}=G(\mathbb{Q})_{+} \mathrm{K}^{p} \mathrm{~K}_{p} / G(\mathbb{Q})_{+} \mathrm{K}^{p} \mathrm{~K}_{p}^{\circ}=\mathrm{K}_{p} /\left(G(\mathbb{Q})_{+} \mathrm{K}^{\circ} \cap \mathrm{K}_{p}\right)=\mathrm{K}_{p} / \mathrm{K}_{p}^{\circ}$

As $\mathrm{K}_{p} / \mathrm{K}_{p}^{\circ}$ transitively on the geometric fibres of

$$
\operatorname{Sh}_{\mathrm{K}^{\circ}}(G, X) \rightarrow \operatorname{Sh}_{\mathrm{K}}(G, X)
$$

this implies that (4.3.8) is a $\mathrm{K}_{p} / \mathrm{K}_{p}^{\circ}$-torsor which becomes trivial over $\overline{\mathbb{Q}}_{p}$. Lemma 4.3 .2 together with [20, Thm. 2.6.3, which describes the action of $\operatorname{Gal}(\overline{\mathbb{Q}} / \mathrm{E})$ on the geometrically connected components of $\operatorname{Sh}_{K_{p}^{\circ}}(G, X)$, now imply that this torsor actually splits after base changing to an unramified extension of $E$. This proves the Proposition for $\mathrm{K}^{p}$ sufficiently small. 
To show the Proposition for any $\mathrm{K}^{p}$ (still satisfying the smallness assumption imposed in (4.1.1)), Let $\mathrm{K}^{p \prime} \subset \mathrm{K}^{p}$ such that the Proposition holds for $\mathrm{K}^{\prime}=\mathrm{K}^{p \prime} \mathrm{K}_{p}$ and $\mathrm{K}^{\prime \circ}=\mathrm{K}^{p /} \mathrm{K}_{p}^{\circ}$. Then, by what we have shown above together with Proposition 4.2.2, the maps

$$
\mathscr{S}_{\mathrm{K}^{\prime}}(G, X) \rightarrow \mathscr{S}_{\mathrm{K}^{\prime}}(G, X) \rightarrow \mathscr{S}_{\mathrm{K}}(G, X)
$$

are finite étale. Since the composite of these maps factors through $\mathscr{S}_{\mathrm{k}}(G, X)$, it follows that

$$
\mathscr{S}_{K^{\circ}}(G, X) \rightarrow \mathscr{S}_{\mathrm{K}}(G, X)
$$

is finite étale.

Corollary 4.3.9. The geometrically connected components of $\mathscr{S}_{\mathrm{K}_{p}^{\circ}}(G, X)$ are defined over the maximal extension of $\mathrm{E}$ that is unramified over primes dividing $p$.

Proof. This follows from 4.3.2 as well as 20, Thm. 2.6.3, which describes the action of $\operatorname{Gal}(\overline{\mathbb{Q}} / \mathrm{E})$ on the geometrically connected components of $\operatorname{Sh}_{\mathrm{K}_{p}^{\circ}}(G, X)$.

4.3.10. The pullback of the torsor $\pi$ introduced in Theorem 4.2.7, by the morphism $\mathscr{S}_{K^{\circ}}(G, X) \rightarrow \mathscr{S}_{\mathrm{K}}(G, X)$ produces a $\mathcal{G}$-torsor

$$
\pi^{\circ}: \widetilde{\mathscr{S}}_{\mathrm{K}}(G, X) \rightarrow \mathscr{S}_{\mathrm{K}}(G, X) .
$$

We conjecture that this $\mathcal{G}$-torsor has a reduction to a $\mathcal{G}^{\circ}$-torsor, although we are unable to prove this.

\subsection{Twisting Abelian Varieties.}

4.4.1. In the next three subsections, we deduce the consequences of the above results for Shimura varieties of abelian type. Many of the arguments of 43 \$3 in the hyperspecial case go over unchanged, so we discuss in detail only those points which do not. One of these concerns the definition of the action of $G^{\text {ad }}(\mathbb{Q})^{+}$on the models $\mathscr{S}_{\mathrm{K}_{p}}(G, X)$ constructed above. In the hyperspecial case the models $\mathscr{S}_{\mathrm{K}_{p}}(G, X)$ satisfy Milne's extension property. This implies the action of $G^{\text {ad }}(\mathbb{Q})^{+}$ on the generic fibre extends to the whole model, and it sufficed in 43 . to give a description of this action on the level of abelian varieties up to isogeny. In the case considered here, we do not have an analogue of the extension property, and we need to give a direct description of the action of $G^{\text {ad }}(\mathbb{Q})^{+}$. This requires a refined form of the twisting construction in $\S 3.1$ of loc. cit.

4.4.2. Let $A$ be a commutative ring with identity, $Z$ a flat, affine group scheme over $\operatorname{Spec} A$, and $\mathcal{P}$ a $Z$-torsor. Note that by flat base change, the coherent cohomology of $\mathcal{P}$ vanishes, so $\mathcal{P}$ is affine. We write $\mathcal{O}_{Z}$ and $\mathcal{O}_{\mathcal{P}}$ for the affine rings of $Z$ and $\mathcal{P}$ respectively. If $M$ is an $A$-module, a $Z$-action on $M$ is a map of fppf sheaves $Z \rightarrow \underline{\text { Aut }} M$. Giving a $Z$-action on $M$ is equivalent to giving $M$ the structure of

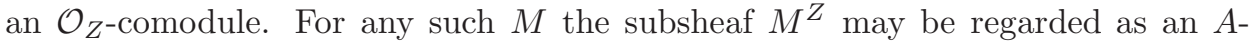
submodule of $M$ by descent.

Lemma 4.4.3. With the notation above, the natural map

$$
\left(M \otimes_{A} \mathcal{O}_{\mathcal{P}}\right)^{Z} \otimes_{A} \mathcal{O}_{\mathcal{P}} \rightarrow M \otimes_{A} \mathcal{O}_{\mathcal{P}}
$$

is an isomorphism 
Proof. Let $\pi_{1}, \pi_{2}$ be the morphisms $Z \times_{\operatorname{Spec} A} \mathcal{P} \rightarrow \mathcal{P}$ given by sending $(z, h)$ to $z h$ and $h$ respectively. A semi-linear action of $Z$ on an $\mathcal{O}_{\mathcal{P}}$ module $N$ gives rise to an isomorphism $\pi_{1}^{*}(N) \cong \pi_{2}^{*}(N)$, which via the isomorphism $Z \times \mathcal{P} \cong \mathcal{P} \times \mathcal{P}$ is nothing but a descent datum for the morphism $\mathcal{P} \rightarrow \operatorname{Spec} A$. We apply this to $N=M \otimes_{A} \mathcal{O}_{\mathcal{P}}$. The lemma now follows by faithfully flat descent, since $Z$ is flat over $A$ and hence so is $\mathcal{P}$.

4.4.5. We now suppose that $Z$ is of finite type, and that $A \subset \mathbb{Q}$. For $S$ a scheme we define the $A$-isogeny category of abelian schemes over $S$ to be the category obtained from the category of abelian schemes over $S$ by tensoring the Hom groups by $\otimes_{\mathbb{Z}} A$. An object $\mathcal{A}$ in this category is called an abelian scheme up to A-isogeny over $S$. For $T$ an $S$-scheme we set $\mathcal{A}(T)=\operatorname{Hom}_{S}(T, \mathcal{A}) \otimes_{\mathbb{Z}} A$.

Let $\mathcal{A}$ be an abelian scheme up to $A$-isogeny over $S$. Denote by $\underline{\operatorname{Aut}}_{A}(\mathcal{A})$ the $A$-group whose points in an $A$-algebra $R$ are given by

$$
\text { Aut }_{A}(\mathcal{A})(R)=\left(\left(\operatorname{End}_{S} \mathcal{A}\right) \otimes_{\mathbb{Z}} R\right)^{\times} .
$$

Let $Z$ and $\mathcal{P}$ be as above, and suppose that we are given a map of $A$-groups $Z \rightarrow \underline{\operatorname{Aut}}_{A}(\mathcal{A})$. We define a pre-sheaf $\mathcal{A}^{\mathcal{P}}$ in the fppf topology of $S$ by setting

$$
\mathcal{A}^{\mathcal{P}}(T)=\left(\mathcal{A}(T) \otimes_{\mathbb{Q}} \mathcal{O}_{\mathcal{P}}\right)^{Z} .
$$

Lemma 4.4.6. $\mathcal{A}^{\mathcal{P}}$ is a sheaf, represented by an abelian scheme up to A-isogeny.

Proof. By a result of Moret-Bailly, [55] Thm. 1.6, there exists a finite, integral, torsion free $A$-algebra $A^{\prime}$ such that $\mathcal{P}\left(A^{\prime}\right)$ is non-empty. Specializing (4.4.4) by the map $\mathcal{O}_{\mathcal{P}} \rightarrow A^{\prime}$ we obtain an isomorphism $\mathcal{A}^{\mathcal{P}} \otimes_{A} A^{\prime} \cong \mathcal{A} \otimes_{A} A^{\prime}$. Since $A^{\prime}$ is a free $A$-module $\mathcal{A} \otimes_{A} A^{\prime}$ is an abelian scheme up to $A$-isogeny.

We may assume that $\operatorname{Fr} A^{\prime}$ is Galois over $\mathbb{Q}$, when $\mathcal{A}^{\mathcal{P}}$ is the $\operatorname{Gal}\left(\operatorname{Fr} A^{\prime} / \mathbb{Q}\right)$ invariants of $\mathcal{A}^{\mathcal{P}} \otimes_{A} A^{\prime}$. Hence $\mathcal{A}^{\mathcal{P}}$ is the kernel of a map of abelian schemes up to $A$-isogeny. Write this map as $n^{-1} \cdot f$ where $n$ is an integer which is invertible in $A$, and $f$ is a map of abelian schemes. Let $B$ be the connected component of the identity of $\operatorname{ker}(f)$, and view $B$ as an abelian scheme up to $A$-isogeny. The cokernel of the natural inclusion $B \subset \mathcal{A}^{\mathcal{P}}$ is a torsion sheaf, so the natural map $B \otimes_{A} A^{\prime} \rightarrow \mathcal{A} \otimes_{A} A^{\prime}$ induced by (4.4.4) is an isomorphism, which implies that $B=\mathcal{A}^{\mathcal{P}}$.

4.4.7. Keeping the above assumptions, denote by $\mathcal{A}^{*}$ the dual abelian scheme. By an $A$-polarization, we mean an isomorphism $\mathcal{A} \stackrel{\sim}{\rightarrow} \mathcal{A}^{*}$ of abelian schemes up to $A$ isogeny, some multiple of which can be realized as a polarization of abelian schemes. Two $A$-polarizations are said to be equivalent if they differ by a multiplication by an element of $A^{\times}$. A weak $A$-polarization is an equivalence class of $A$-polarizations.

Let $c: Z \rightarrow \mathbb{G}_{m}$ be a character. We will denote by $\mathcal{A}(c)$ the abelian scheme up to $A$-isogeny $\mathcal{A}$ equipped with the map $Z \rightarrow \underline{\operatorname{Aut}}_{A} \mathcal{A}$ obtained by multiplying the natural action by $c$. Let $\lambda: \mathcal{A} \rightarrow \mathcal{A}^{*}$ be a weak $A$-polarization. We have a canonical map $Z \rightarrow \underline{\operatorname{Aut}}_{A}\left(\mathcal{A}^{*}\right)$. We say that $\lambda$ is a $c$-polarization if the induced map $\mathcal{A} \rightarrow \mathcal{A}^{*}(c)$ is compatible with $Z$-actions. The same argument as in [4] Lemma 3.1.5 proves the following

Lemma 4.4.8. There is a natural isomorphism $\left(\mathcal{A}^{*}\right)^{\mathcal{P}} \cong \mathcal{A}^{\mathcal{P} *}$. If $\lambda: \mathcal{A} \rightarrow \mathcal{A}^{*}$ is a c-polarization, then there is a unique weak A-polarization $\lambda^{\mathcal{P}}: \mathcal{A}^{\mathcal{P}} \rightarrow \mathcal{A}^{\mathcal{P} *}$ such 
that the diagram

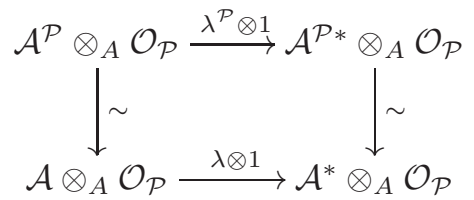

commutes up to an element of $\mathcal{O}_{\mathcal{P}}^{\times}$. Here the map on the right is obtained by composing $\mathcal{A}^{\mathcal{P} *} \cong\left(\mathcal{A}^{*}\right)^{\mathcal{P}}$ with the isomorphism of (4.4.4).

\subsection{The adjoint group action.}

4.5.1. We now return to the assumptions and notations of 4.2 In particular, we have an embedding of Shimura data $(G, X) \subset\left(\mathrm{GSp}, S^{ \pm}\right)$where $\operatorname{GSp}=\operatorname{GSp}\left(V_{\mathbb{Q}}\right)$ and $V_{\mathbb{Q}}$ is equipped with a lattice $V_{\mathbb{Z}}$, and an embedding

$$
\operatorname{Sh}_{\mathrm{K}}(G, X) \hookrightarrow \operatorname{Sh}_{\mathrm{K}^{\prime}}\left(\mathrm{GSp}, S^{ \pm}\right) .
$$

Let $\mathcal{B}$ be an abelian scheme up to $\mathbb{Z}_{(p)}$-isogeny over a $\mathbb{Z}_{(p)}$-scheme $T$. Set $\widehat{V}^{p}(\mathcal{B})=$ $\lim _{\longleftarrow \nmid n} \mathcal{B}[n]$ and

$$
\widehat{V}^{p}(\mathcal{B})_{\mathbb{Z}_{(p)}}=\widehat{V}^{p}(\mathcal{B}) \otimes_{\mathbb{Z}} \mathbb{Z}_{(p)}=\widehat{V}^{p}(\mathcal{B}) \otimes_{\mathbb{Z}} \mathbb{Q}
$$

Suppose $\mathcal{B}$ has dimension $\operatorname{dim}_{\mathbb{Q}} V_{\mathbb{Q}} / 2$ and its equipped with a weak $\mathbb{Z}_{(p)}$-isogeny $\lambda$. We denote by $\underline{\operatorname{Isom}}\left(V_{\mathbb{A}_{f}^{p}}, \widehat{V}^{p}(\mathcal{B})_{\mathbb{Q}}\right)$ the pro-étale sheaf of isomorphisms $V_{\mathbb{A}_{f}^{p}} \cong \widehat{V}^{p}(\mathcal{B})_{\mathbb{Q}}$ which are compatible with the pairings induced by $\psi$ and $\lambda$ up to a $\mathbb{A}_{f}^{p, \times}$-scalar. Then $\underline{\operatorname{Isom}}\left(V_{\mathbb{A}_{f}^{p}}, \widehat{V}^{p}(\mathcal{B})_{\mathbb{Q}}\right) / \mathrm{K}^{p^{\prime}}$ is an étale sheaf.

A point $x \in \mathscr{S}_{\mathrm{K}^{\prime}}\left(\mathrm{GSp}, S^{ \pm}\right)(T)$ corresponds to a triple $\left(\mathcal{A}_{x}, \lambda_{x}, \varepsilon_{x}^{p}\right)$, where $\mathcal{A}_{x}$ is an abelian scheme over $T$ up to $\mathbb{Z}_{(p)}$-isogeny, equipped with a weak $\mathbb{Z}_{(p)}$-polarization $\lambda_{x}$, and

$$
\varepsilon_{x}^{p} \in \Gamma\left(T, \underline{\operatorname{Isom}}\left(V_{\mathbb{A}_{f}^{p}}, \widehat{V}^{p}\left(\mathcal{A}_{x}\right)_{\mathbb{Q}}\right) / \mathrm{K}^{p \prime}\right) .
$$

If $x \in \mathscr{S}_{\mathrm{K}}(G, X)(T)$, then as in 43] (3.2.4), $\varepsilon_{x}^{p}$ can be promoted to a section $\varepsilon_{x}^{p} \in \Gamma\left(T, \underline{\operatorname{Isom}}\left(V_{\mathbb{A}_{f}^{p}}, \widehat{V}^{p}\left(\mathcal{A}_{x}\right)_{\mathbb{Q}}\right) / \mathrm{K}^{p}\right)$. Similarly, if $x \in \mathscr{S}_{\mathrm{K}_{p}}(G, X)(T)$, then we obtain an element

$$
\varepsilon_{x}^{p} \in \lim _{\leftarrow \mathrm{K}^{p}} \Gamma\left(T, \underline{\operatorname{Isom}}\left(V_{\mathbb{A}_{f}^{p}}, \widehat{V}^{p}\left(\mathcal{A}_{x}\right)_{\mathbb{Q}}\right) / \mathrm{K}^{p}\right) .
$$

We denote by $Z=Z_{G_{\mathbb{Z}_{(p)}}}$ the closure in $G_{\mathbb{Z}_{(p)}}$ of the center of the $\mathbb{Q}$-group $G$.

Lemma 4.5.2. If $x \in \mathscr{S}_{\mathrm{K}}(G, X)(T)$, then there is a natural embedding

$$
Z \hookrightarrow \underline{\mathrm{Aut}}_{\mathbb{Z}_{(p)}}\left(\mathcal{A}_{x}\right)
$$

Proof. It suffices to construct the embedding for the universal point with $T=$ $\mathscr{S}_{\mathrm{K}}(G, X)$, and by [23] I, 2.7 it suffices to consider $T=\operatorname{Sh}_{\mathrm{K}}(G, X)$.

By [43] Lemma 3.2.2, 3.4.1 there is a natural embedding $Z \otimes_{\mathbb{Z}_{(p)}} \mathbb{Q} \hookrightarrow \underline{\operatorname{Aut}}_{\mathbb{Q}}\left(\mathcal{A}_{x}\right)$. For $y \in \operatorname{Sh}_{K}(G, X)(\mathbb{C})$ this embedding specializes to the one induced by the natural action of $G$ on $H_{1}\left(\mathcal{A}_{y}(\mathbb{C}), \mathbb{Q}\right)$, obtained by choosing a lift $\tilde{y} \in X \times G\left(\mathbb{A}_{f}\right)$ of $y$. In particular, since $G_{\mathbb{Z}_{(p)}}$ is a subgroup of $\mathrm{GL}\left(V_{\mathbb{Z}_{(p)}}\right)$, we obtain maps

$$
Z \rightarrow \underline{\operatorname{Aut}}_{\mathbb{Z}_{(p)}}\left(\mathcal{A}_{y}\right) \rightarrow \mathrm{GL}\left(V_{\mathbb{Z}_{(p)}}\right) .
$$

As the composite is a closed embedding, so is the first map. Hence we get an embedding $Z \hookrightarrow \underline{\text { Aut }}_{\mathbb{Z}_{(p)}}\left(\mathcal{A}_{x}\right)$. 
4.5.3. Let $G_{\mathbb{Z}_{(p)}}^{\text {ad }}=G_{\mathbb{Z}_{(p)}} / Z_{G_{\mathbb{Z}_{(p)}}}, \gamma \in G_{\mathbb{Z}_{(p)}}\left(\mathbb{Z}_{(p)}\right)$ and $\mathcal{P}$ the fibre of $G_{\mathbb{Z}_{(p)}} \rightarrow G_{\mathbb{Z}_{(p)}}^{\text {ad }}$ over $\gamma$. Then $\mathcal{P}$ is a $Z_{G_{\mathbb{Z}_{(p)}}}$-torsor. Fix a Galois extension $F / \mathbb{Q}$ such that $\mathcal{P}$ admits an $\mathcal{O}_{F,(p)}=\mathcal{O}_{F} \otimes_{\mathbb{Z}} \mathbb{Z}_{(p)}$-point $\tilde{\gamma}$. Such a point exists by the result of Moret-Bailly used in the proof of lemma 4.4.6 above. Applying lemma 4.5.2 and specializing (4.4.4) by $\tilde{\gamma}$ we obtain a $\mathbb{Z}_{(p) \text {-isogeny }}$

$$
\iota_{\tilde{\gamma}}: \mathcal{A}_{x}^{\mathcal{P}} \otimes_{\mathbb{Z}_{(p)}} \mathcal{O}_{F,(p)} \stackrel{\sim}{\rightarrow} \mathcal{A}_{x} \otimes_{\mathbb{Z}_{(p)}} \mathcal{O}_{F,(p)} .
$$

Lemma 4.5.4. The composite

$$
V_{\mathbb{A}_{f}^{p}} \otimes_{\mathbb{Q}} F \stackrel{\tilde{\gamma}^{-1}}{\longrightarrow} V_{\mathbb{A}_{f}^{p}} \otimes_{\mathbb{Q}} F \stackrel{\varepsilon_{x}^{p}}{\longrightarrow} \widehat{V}^{p}\left(\mathcal{A}_{x}\right) \otimes_{\mathbb{Q}} F \stackrel{i_{\hat{\gamma}}^{-1}}{\longrightarrow} \widehat{V}^{p}\left(\mathcal{A}_{x}^{\mathcal{P}}\right) \otimes_{\mathbb{Q}} F
$$

is $\operatorname{Gal}(F / \mathbb{Q})$-invariant and induces a section

$$
\varepsilon_{x}^{p, \mathcal{P}} \in \Gamma\left(T, \underline{\operatorname{Isom}}\left(V_{\mathbb{A}_{f}^{p}}, \widehat{V}^{p}\left(\mathcal{A}_{x}^{\mathcal{P}}\right)_{\mathbb{Q}}\right) / \gamma \mathrm{K}^{p} \gamma^{-1}\right)
$$

Proof. This is identical to the proof of [43] lemma 3.4.

4.5.6. We recall the notation of $[20$. Let $H$ be a group equipped with an action of a group $\Delta$, and $\Gamma \subset H$ a $\Delta$-stable subgroup. Suppose given a $\Delta$-equivariant map $\varphi: \Gamma \rightarrow \Delta$ where $\Delta$ acts on itself by inner automorphisms, and suppose that for $\gamma \in \Gamma, \varphi(\gamma)$ acts on $H$ as inner conjugation by $\gamma$. Then the elements of the form $\left(\gamma, \varphi(\gamma)^{-1}\right)$ form a normal subgroup of the semi-direct product $H \rtimes \Delta$. We denote by $H *_{\Gamma} \Delta$ the quotient of $H \rtimes \Delta$ by this subgroup.

For a subgroup $H \subset G(\mathbb{R})$ denote by $H_{+}$the preimage in $H$ of $G^{\text {ad }}(\mathbb{R})^{+}$, the connected component of the identity in $G^{\text {ad }}(\mathbb{R})$. As usual, we write $G^{\text {ad }}(\mathbb{Q})^{+}=$ $G^{\text {ad }}(\mathbb{Q}) \cap G^{\text {ad }}(\mathbb{R})^{+}$.

There is an action of $G^{\text {ad }}(\mathbb{Q})^{+}$on $\operatorname{Sh}(G, X)$ induced by the action by conjugation of $G$ on itself. Combining this with the action of $G\left(\mathbb{A}_{f}\right)$ on $\operatorname{Sh}(G, X)$, gives rise to a right action of

$$
\mathscr{A}(G):=G\left(\mathbb{A}_{f}\right) / Z(\mathbb{Q})^{-} *_{G(\mathbb{Q})_{+} / Z(\mathbb{Q})} G^{\mathrm{ad}}(\mathbb{Q})^{+} .
$$

on $\operatorname{Sh}(G, X)$ where $Z(\mathbb{Q})^{-}$denotes the closure of $Z_{G}(\mathbb{Q})$ in $G\left(\mathbb{A}_{f}\right)$.

Let $G(\mathbb{Q})_{+}^{-}$denote the closure of $G(\mathbb{Q})_{+}$in $G\left(\mathbb{A}_{f}\right)$ and set

$$
\mathscr{A}(G)^{\circ}=G(\mathbb{Q})_{+}^{-} / Z(\mathbb{Q})^{-} *_{G(\mathbb{Q})_{+} / Z(\mathbb{Q})} G^{\mathrm{ad}}(\mathbb{Q})^{+} .
$$

This group depends only on $G^{\text {der }}$ and not on $G$; it is equal to the completion of $G^{\text {ad }}(\mathbb{Q})^{+}$with respect to the topology whose open sets are images of congruence subgroups in $G^{\text {der }}(\mathbb{Q})[20$ 2.7.12. This definition will be used in the next subsection.

The action of $G^{\text {ad }}(\mathbb{Q})^{+}$on $\operatorname{Sh}(G, X)$ induces an action of the group $G^{\text {ad }}\left(\mathbb{Z}_{(p)}\right)^{+}$ on $\mathrm{Sh}_{\mathrm{K}_{p}}(G, X)$. This gives rise to an action of

$$
\mathscr{B}\left(G_{\mathbb{Z}_{(p)}}\right):=G\left(\mathbb{A}_{f}^{p}\right) / Z\left(\mathbb{Z}_{(p)}\right)^{-} *_{G\left(\mathbb{Z}_{(p)}\right)+/ Z\left(\mathbb{Z}_{(p)}\right)} G^{\mathrm{ad}}\left(\mathbb{Z}_{(p)}\right)^{+}
$$

on $\operatorname{Sh}_{\mathfrak{K}_{p}}(G, X)$. Here $Z\left(\mathbb{Z}_{(p)}\right)^{-}$denotes the closure of $Z\left(\mathbb{Z}_{(p)}\right)$ in $G\left(\mathbb{A}_{f}^{p}\right)$.

Lemma 4.5.7. Let $\gamma \in G^{\mathrm{ad}}\left(\mathbb{Z}_{(p)}\right)^{+}$, and $\mathcal{P}$ the fibre of $G_{\mathbb{Z}_{(p)}} \rightarrow G_{\mathbb{Z}_{(p)}}^{\mathrm{ad}}$ over $\gamma$. For $T$ a $\mathbb{Z}_{(p)}$-scheme and $x \in \mathscr{S}_{\mathrm{K}_{p}}(G, X)(T)$, the assignment

$$
\left(\mathcal{A}_{x}, \lambda_{x}, \varepsilon_{x}^{p}\right) \mapsto\left(\mathcal{A}_{x}^{\mathcal{P}}, \lambda_{x}^{\mathcal{P}}, \varepsilon_{x}^{p, \mathcal{P}}\right)
$$

induces a map

$$
\mathscr{S}_{\mathrm{K}_{p}}(G, X) \rightarrow \mathscr{S}_{\mathrm{K}_{p}}(G, X)
$$

whose generic fibre agrees with the map induced by conjugation by $\gamma$. 
Combining the $G^{\mathrm{ad}}\left(\mathbb{Z}_{(p)}\right)^{+}$-action with the natural action of $G\left(\mathbb{A}_{f}^{p}\right)$ on $\mathscr{S}_{\mathrm{K}_{p}}(G, X)$ induces an action of $\mathscr{B}\left(G_{\mathbb{Z}_{(p)}}\right)$ on $\mathscr{S}_{\mathrm{K}_{p}}(G, X)$.

Proof. The assignment induces a map

$$
\mathscr{S}_{\mathrm{K}_{p}}(G, X) \rightarrow \mathscr{S}_{\mathrm{K}_{p}^{\prime}}\left(\mathrm{GSp}, S^{ \pm}\right) .
$$

The same argument as in 43 Lemma 3.2.6 shows that on generic fibres this map factors through $\mathscr{S}_{\mathrm{K}_{p}}(G, X)$ and induces the map obtained from the conjugation by $\gamma$. The lemma follows from the definition of $\mathscr{S}_{\mathrm{K}_{p}}(G, X)$ as the normalization of the closure of $\operatorname{Sh}_{\mathrm{K}_{p}}(G, X)$ in $\mathscr{S}_{\mathrm{K}_{p}^{\prime}}\left(\mathrm{GSp}, S^{ \pm}\right)$.

The final claim follows from the analogous result on generic fibres, which is easily checked on complex points.

4.5.8. Recall that in Theorem 4.2.7 we defined a $G_{\mathbb{Z}_{(p)}}$-torsor $\widetilde{\mathscr{S}_{\mathrm{K}}}(G, X)_{\mathcal{O}_{E}}$, and we denote by $\widetilde{\mathscr{S}_{k}}=\widetilde{\mathscr{S}}_{\mathrm{K}_{p}}(G, X)$, its pullback to $\mathscr{S}_{\mathrm{K}_{p}}(G, X)$. Let $\widetilde{\mathscr{S}}_{\mathrm{K}_{p}}^{\text {ad }}$ be the $G_{\mathbb{Z}_{(p)}}^{\text {ad }}$ torsor obtained from $\widetilde{\mathscr{S}}_{\mathrm{K}_{p}}$. We remark that the map $q$ in (4.2.8) obviously factors through $\widetilde{\mathscr{S}_{\mathrm{K}}^{\mathrm{ad}}}$.

We will show that the action of $\mathscr{B}\left(G_{\mathbb{Z}_{(p)}}\right)$ on $\mathscr{S}_{\mathrm{K}_{p}}(G, X)$ defined above, can be lifted to $\widetilde{\mathscr{S}_{\mathrm{K}_{p}}^{\mathrm{ad}}}$.

Lemma 4.5.9. The action of $G^{\text {ad }}\left(\mathbb{Z}_{(p)}\right)^{+}$on $\mathscr{S}_{\mathrm{K}_{p}}(G, X)$ lifts to an action on $\widetilde{\mathscr{S}_{\mathrm{K}_{p}}}$ as a $G_{\mathbb{Z}_{p}}^{\mathrm{ad}}$-torsor. If we equip $\mathrm{M}_{G, X}^{\mathrm{loc}}$ with the trivial $\mathscr{B}\left(G_{\mathbb{Z}_{(p)}}\right)$-action, the maps in the diagram of $\mathcal{O}_{E}$-schemes

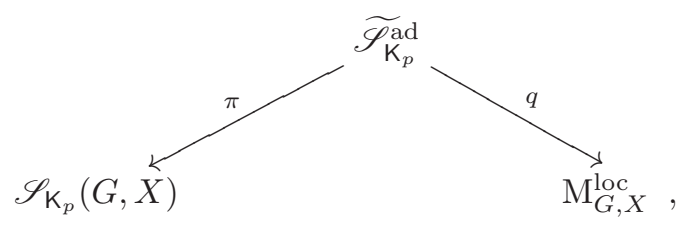

are $\mathscr{B}\left(G_{\mathbb{Z}_{(p)}}\right)$-equivariant. Moreover any sufficiently small $\mathrm{K}^{p} \subset G\left(\mathbb{A}_{f}^{p}\right)$ acts freely on $\widetilde{\mathrm{S}_{\mathrm{K}}}$, and the map

$$
\widetilde{\mathscr{S}_{\mathrm{K}}} / \mathrm{K}^{p} \rightarrow \mathrm{M}_{G, X}^{\mathrm{loc}}
$$

induced by $q$ is smooth of relative dimension $\operatorname{dim} G^{\mathrm{ad}}$.

Proof. We begin by defining the action of $G^{\text {ad }}\left(\mathbb{Z}_{(p)}\right)^{+}$on $\widetilde{\mathscr{S}_{\mathrm{K}_{p}}^{\text {ad }}}$. Let $S$ be an $\mathcal{O}_{E^{-}}$ scheme, and $(x, f) \in \widetilde{\mathscr{S}}_{\mathrm{K}_{p}}(S)$ where $x \in \mathscr{S}_{\mathrm{K}_{p}}(G, X)(S)$, and $f$ is a map $f: V_{\mathbb{Z}_{p}}^{\vee} \otimes$ $\mathcal{O}_{S} \cong R^{1} f_{*} \Omega_{\mathcal{A}_{x} / S}^{1}$. Let $\gamma \in G^{\text {ad }}\left(\mathbb{Z}_{(p)}\right)^{+}$, and $\mathcal{P}$ the corresponding $Z$-torsor. Choose a number field $F$, Galois over $\mathbb{Q}$, and a section $\tilde{\gamma} \in \mathcal{P}\left(\mathcal{O}_{F}\right)$. For $\sigma \in \operatorname{Gal}(F / \mathbb{Q})$ set $c_{\tilde{\gamma}}(\sigma)=\sigma(\tilde{\gamma}) \gamma^{-1}$. Consider the composite

$$
\tilde{\gamma}(f): V_{\mathbb{Z}_{p}}^{\vee} \otimes \mathcal{O}_{S} \otimes \mathcal{O}_{F} \underset{f}{\stackrel{\sim}{\longrightarrow}} R^{1} f_{*} \Omega_{\mathcal{A}_{x} / S}^{1} \otimes \mathcal{O}_{F} \underset{l_{\tilde{\gamma}}^{-1}}{\stackrel{\sim}{\longrightarrow}} R^{1} f_{*} \Omega_{\mathcal{A}_{x}^{\mathcal{P}} / S}^{1} \otimes \mathcal{O}_{F} .
$$

Then $(\gamma(x), \tilde{\gamma}(f)) \in \widetilde{\mathscr{S}}_{\mathrm{K}_{p}}\left(S \otimes \mathcal{O}_{F}\right)$. Since $\sigma\left(\iota_{\tilde{\gamma}}\right)=c_{\tilde{\gamma}}(\sigma)^{-1} \iota_{\tilde{\gamma}}$ for $\sigma \in \operatorname{Gal}(F / \mathbb{Q})$ by 43, Lemma 3.4.3, $(\gamma(x), \tilde{\gamma}(f))$ induces a point of $\widetilde{\mathscr{S}}_{\mathrm{K}_{p}}^{\text {ad }}(S)$, which depends only on the image of $(x, f)$ in $\widetilde{\mathscr{S}_{\mathrm{K}}}$ ad $(S)$ and on $\gamma$. 
This shows that $(x, f) \mapsto(\gamma(x), \gamma(f))$ induces an action of $G^{\text {ad }}\left(\mathbb{Z}_{(p)}\right)^{+}$on $\widetilde{\mathscr{S}_{\mathrm{K}_{p}}^{\text {ad }}}$, lifting the action on $\mathscr{S}_{\mathrm{K}_{p}}(G, X)$. That the map $q$ is $G^{\text {ad }}\left(\mathbb{Z}_{(p)}\right)^{+}$-equivariant, follows from the fact that the map $\iota_{\tilde{\gamma}}^{-1}$ in the definition of $\gamma(f)$ respects Hodge filtrations, as it arises from a map of abelian varieties.

Now lift the action of $h \in G\left(\mathbb{A}_{f}^{p}\right)$ to $\widetilde{\mathscr{S}}_{\mathrm{K}_{p}}$ by sending $(x, f)$ to $(h(x), f)$. It remains to show that this, together with the action of $G^{\text {ad }}\left(\mathbb{Z}_{(p)}\right)^{+}$, defined above, defines an action of $\mathscr{B}\left(G_{\mathbb{Z}_{(p)}}\right)$ in $\widetilde{\mathscr{S}_{\mathrm{K}_{p}}^{\text {ad }}}$. To do this we have to check it defines an action of $G\left(\mathbb{A}_{f}^{p}\right) \rtimes G^{\text {ad }}\left(\mathbb{Z}_{(p)}\right)^{+}$such that $G\left(\mathbb{Z}_{(p)}\right)+$ acts trivially. If $h \in G\left(\mathbb{A}_{f}^{p}\right)$ and $g \in G^{\mathrm{ad}}\left(\mathbb{Z}_{(p)}\right)^{+}$, then as $G\left(\mathbb{A}_{f}^{p}\right) \rtimes G^{\mathrm{ad}}\left(\mathbb{Z}_{(p)}\right)^{+}$acts on $\mathscr{S}_{\mathrm{K}_{p}}(G, X)$, we have $g\left(h\left(g^{-1}(x, f)\right)\right)=g\left(h\left(\left(g^{-1}(x), g^{-1}(f)\right)\right)\right)=g\left(h\left(g^{-1}(x)\right), g^{-1}(f)\right)=\left(g h g^{-1}(x), f\right)$, which implies that $G\left(\mathbb{A}_{f}^{p}\right) \rtimes G^{\text {ad }}\left(\mathbb{Z}_{(p)}\right)^{+}$acts on $\widetilde{\mathscr{S}}_{\mathrm{K}_{p}}$.

If $\gamma$ lifts to $\tilde{\gamma} \in G\left(\mathbb{Z}_{(p)}\right)_{+}$, then $\left(\tilde{\gamma}, \tilde{\gamma}^{-1}\right)(x)=x$. More precisely, $\left(\tilde{\gamma}, \tilde{\gamma}^{-1}\right)(x)$ corresponds to the triple $\left(\mathcal{A}_{x}^{\mathcal{P}}, \varepsilon_{x}^{p, \mathcal{P}} \circ \tilde{\gamma}, \lambda_{x}^{\mathcal{P}}\right)$, and $\iota_{\tilde{\gamma}}: \mathcal{A}_{x}^{\mathcal{P}} \cong \mathcal{A}_{x}$ induces an isomorphism of this triple with $\left(\mathcal{A}_{x}, \varepsilon_{x}^{p}, \lambda_{x}\right)$. Similarly, $\iota \tilde{\gamma}$ intertwines the isomorphisms $f$ and $\gamma(f)$, which proves that $G\left(\mathbb{Z}_{(p)}\right)+$ acts trivially on $\widetilde{\mathscr{S}_{k}}$.

The final statement follows immediately from the construction of $\widetilde{\mathscr{S}}_{\mathrm{K}_{p}}$.

4.6. Shimura varieties of abelian type. We continue to use the notation and assumptions of the previous subsection.

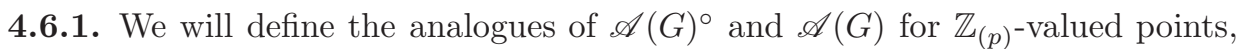
but we need some preparation.

Suppose $S$ is an affine $\mathbb{Q}$-scheme, and let $S_{\mathbb{Z}_{p}}$ be a flat, affine $\mathbb{Z}_{p}$-scheme, with generic fibre $S \otimes \mathbb{Q}_{p}$. Then there is a canonical $\mathbb{Z}_{(p)}$-scheme $S_{\mathbb{Z}_{(p)}}$ with generic fibre $S$ such that $S_{\mathbb{Z}_{(p)}} \otimes_{\mathbb{Z}_{(p)}} \mathbb{Z}_{p}=S_{\mathbb{Z}_{p}}$. Indeed $S_{\mathbb{Z}_{(p)}}$ is the spectrum of the ring obtained by intersecting the global functions on $S_{\mathbb{Z}_{p}}$ and $S$ inside those on $S \otimes \mathbb{Q}_{p}$.

Recall the smooth model $G_{\mathbb{Z}_{(p)}}$ is defined by a point $x \in \mathcal{B}\left(G, \mathbb{Q}_{p}\right)$, and that we set $G_{\mathbb{Z}_{(p)}}^{\text {ad }}=G_{\mathbb{Z}_{(p)}} / Z_{\mathbb{Z}_{(p)}}$. Let $G_{\mathbb{Z}_{(p)}}^{\text {der }}$ be the closure of $G^{\text {der }}$ in $G_{\mathbb{Z}_{(p)}}$. We denote by $G^{\circ}=G_{\mathbb{Z}_{(p)}}^{\circ}$, the connected component of the identity. It is the parahoric group scheme attached to $x$. More precisely, $x$ defines a parahoric group scheme over $\mathbb{Z}_{p}$, which descends to $\mathbb{Z}_{(p)}$ via the general construction described above. Similarly, let $G^{\text {ado }}=G_{\mathbb{Z}_{(p)}}^{\text {ado }}$ and $G^{\text {dero }}=G_{\mathbb{Z}_{(p)}}^{\text {dero }}$ be the parahoric models of $G^{\text {ad }}$ and $G^{\text {der }}$ respectively, defined by the image, $x^{\text {ad }}$, of $x$ in $\mathcal{B}\left(G^{\text {ad }}, \mathbb{Q}_{p}\right)=\mathcal{B}\left(G^{\text {der }}, \mathbb{Q}_{p}\right)$. Note that, in general, $G_{\mathbb{Z}_{(p)}}^{\text {ado }}$ is not equal to the neutral component $\left(G_{\mathbb{Z}_{(p)}}^{\text {ad }}\right)^{\circ}$ of $G_{\mathbb{Z}_{(p)}}^{\text {ad }}$, but see (2) below.

Lemma 4.6.2. We have

(1) $G_{\mathbb{Z}_{(p)}}^{\text {der }}$ is the stabilizer of $x^{\text {ad }}$. In particular, $G_{\mathbb{Z}_{(p)}}^{\text {dero }}$ is the connected component of the identity of $G_{\mathbb{Z}_{(p)}}^{\text {der }}$.

(2) Suppose that either the center $Z_{G}$ is connected or that $Z_{G^{\mathrm{der}}}$ has rank prime to $p$. Then $G_{\mathbb{Z}_{(p)}}^{\text {ado }}$ is the connected component of the identity of $G_{\mathbb{Z}_{(p)}}^{\text {ad }}=$ $G_{\mathbb{Z}_{(p)}} / Z_{\mathbb{Z}_{(p)}}$. In particular, there is a map of $\mathbb{Z}_{(p)}$-group schemes $G_{\mathbb{Z}_{(p)}}^{\text {ado }} \rightarrow$ $G_{\mathbb{Z}_{(p)}}^{\text {ad }}$, extending the identity on generic fibres.

Proof. Let $G_{\mathbb{Z}_{p}}^{\text {der }}=G_{\mathbb{Z}_{(p)}}^{\text {der }} \otimes_{\mathbb{Z}_{(p)}} \mathbb{Z}_{p}$. Let $x^{\text {der }} \in \mathcal{B}\left(G^{\text {der }}, \mathbb{Q}_{p}\right) \subset \mathcal{B}\left(G, \mathbb{Q}_{p}\right)$ be the preimage of $x^{\text {ad }}$ under the identification $\mathcal{B}\left(G^{\text {der }}, \mathbb{Q}_{p}\right)=\mathcal{B}\left(G^{\text {ad }}, \mathbb{Q}_{p}\right)$. Then $\mathcal{G}_{x^{\text {der }}} \hookrightarrow$ 
$\mathcal{G} \mathcal{L}\left(V_{Z_{p}}\right)_{\iota_{x} \text { der }}$ is a closed embedding by Proposition 1.3.3, and similarly for $\mathcal{G}_{x^{\mathrm{der}}}^{\mathrm{der}}$. Thus, the closure of $G^{\text {der }}$ in $\mathcal{G}_{x^{\text {der }}}$ is smooth, and coincides with $\mathcal{G}_{x^{\text {der }}}^{\text {der }}$, the group scheme stabilizer of $x^{\text {ad }}$. On the other hand, $\mathcal{G}_{x^{\text {der }}}$ can be naturally identified with $\mathcal{G}_{x}$ (cf. [70 3.4.1). In particular, the closure of $G^{\text {der }}$ in $\mathcal{G}_{x}$ is smooth, and coincides with the group scheme stabilizer of $x^{\text {ad }}$. Now (1), which is the corresponding statement over $\mathbb{Z}_{(p)}$, follows.

Let us consider (2). Note that by the functoriality of the group schemes stabilizing a point of the building, there is always a map $\left(G_{\mathbb{Z}_{(p)}}^{\text {ad }}\right)^{\circ} \rightarrow G_{\mathbb{Z}_{(p)}}^{\text {ado }}$ (see 1.1.3). If $Z_{G}$ is connected (2) follows immediately from Proposition 1.1.4. Suppose that $Z_{G^{\text {der }}}$ has rank prime to $p$. Then (1) together with 1.1.4 applied to $G^{\text {der }}$ implies that $G_{\mathbb{Z}_{(p)}}^{\text {ado }}$ is the quotient of $G_{\mathbb{Z}_{(p)}}^{\text {dero }}$ by the Zariski closure of the center $Z_{G^{\text {der }}}$. This provides a $\operatorname{map} G_{\mathbb{Z}_{(p)}}^{\text {ado }} \rightarrow G_{\mathbb{Z}_{(p)}} / Z_{\mathbb{Z}_{(p)}}=G_{\mathbb{Z}_{(p)}}^{\text {ad }}$ which gives the inverse $G_{\mathbb{Z}_{(p)}}^{\text {ado }} \rightarrow\left(G_{\mathbb{Z}_{(p)}}^{\text {ad }}\right)^{\circ}$.

4.6.3. Let $Z^{\circ}$ denote the Zariski closure of $Z$ in $G^{\circ}$. We denote by $Z^{\circ}\left(\mathbb{Z}_{(p)}\right)^{-}$ the closure of $Z^{\circ}\left(\mathbb{Z}_{(p)}\right)$ in $Z^{\circ}\left(\mathbb{A}_{f}\right)$. Note that the image of $Z^{\circ}\left(\mathbb{Z}_{(p)}\right)^{-}$in $Z^{\circ}\left(\mathbb{A}_{f}^{p}\right)$ coincides with the closure of $Z^{\circ}\left(\mathbb{Z}_{(p)}\right)$ in $Z^{\circ}\left(\mathbb{A}_{f}^{p}\right)$.

Let

$\tilde{\mathscr{A}}\left(G_{\mathbb{Z}_{(p)}}\right)=\left[G\left(\mathbb{A}_{f}^{p}\right) \times G_{\mathbb{Z}_{(p)}}^{\circ}\left(\mathbb{Z}_{p}\right)\right] / Z\left(\mathbb{Z}_{(p)}\right)^{-} *_{G^{\circ}\left(\mathbb{Z}_{(p)}\right)_{+} / Z\left(\mathbb{Z}_{(p)}\right)} G^{\text {ado }}\left(\mathbb{Z}_{(p)}\right)^{+} \subset \mathscr{A}(G)$

and

$$
\tilde{\mathscr{A}}\left(G_{\mathbb{Z}_{(p)}}\right)^{\circ}=G^{\circ}\left(\mathbb{Z}_{(p)}\right) \tilde{+} / Z\left(\mathbb{Z}_{(p)}\right)^{-} *_{G^{\circ}\left(\mathbb{Z}_{(p)}\right)_{+} / Z\left(\mathbb{Z}_{(p)}\right)} G^{\text {ado }}\left(\mathbb{Z}_{(p)}\right)^{+} \subset \mathscr{A}(G)^{\circ}
$$

where $G^{\circ}\left(\mathbb{Z}_{(p)}\right)_{+}^{\sim}$ denotes the closure of $G^{\circ}\left(\mathbb{Z}_{(p)}\right)_{+}$in $G\left(\mathbb{A}_{f}^{p}\right) \times G\left(\mathbb{Z}_{p}\right)$.

We set

$$
\mathscr{A}\left(G_{\mathbb{Z}_{(p)}}\right)=G\left(\mathbb{A}_{f}^{p}\right) / Z\left(\mathbb{Z}_{(p)}\right)^{-} *_{G^{\circ}\left(\mathbb{Z}_{(p)}\right)+/ Z\left(\mathbb{Z}_{(p)}\right)} G^{\mathrm{ado}}\left(\mathbb{Z}_{(p)}\right)^{+}
$$

and

$$
\mathscr{A}\left(G_{\mathbb{Z}_{(p)}}\right)^{\circ}=G^{\circ}\left(\mathbb{Z}_{(p)}\right)_{+}^{-} / Z\left(\mathbb{Z}_{(p)}\right)^{-} *_{G^{\circ}\left(\mathbb{Z}_{(p)}\right)_{+} / Z\left(\mathbb{Z}_{(p)}\right)} G^{\text {ado }}\left(\mathbb{Z}_{(p)}\right)^{+},
$$

where $G^{\circ}\left(\mathbb{Z}_{(p)}\right)_{+}^{-}$is the closure of $G^{\circ}\left(\mathbb{Z}_{(p)}\right)_{+}$in $G\left(\mathbb{A}_{f}^{p}\right)$. Note that the difference between $\mathscr{A}\left(G_{\mathbb{Z}_{(p)}}\right)$ and the group $\mathscr{B}\left(G_{\mathbb{Z}_{(p)}}\right)$ defined in the previous section is that the former is defined using parahoric models of $G$ and $G^{\text {ad }}$.

In what follows, we will assume that either $Z=Z_{G}$ is connected or that $Z_{G^{\text {der }}}$ has rank prime to $p$. Under this assumption, by Lemma 4.6.2 (2) and Lemma 4.5.7 the action of $\mathscr{A}\left(G_{\mathbb{Z}_{(p)}}\right)$ on $\operatorname{Sh}_{\mathrm{K}_{p}}(G, X)$ extends to $\mathscr{S}_{\mathrm{K}_{p}}(G, X)$. As in $\S 4.3$, we denote by $\mathscr{S}_{\mathrm{K}_{p}}(G, X)$ the normalization of $\mathscr{S}_{\mathrm{K}_{p}}(G, X)$ in $\operatorname{Sh}_{\mathrm{K}_{p}}(G, X)$. Then the action of $\mathscr{A}\left(G_{\mathbb{Z}_{(p)}}\right)$ on $\operatorname{Sh}_{K_{p}^{\circ}}(G, X)$ extends to $\mathscr{S}_{\mathrm{K}_{p}^{\circ}}(G, X)$.

Lemma 4.6.4. We have

(1) $\tilde{A}\left(G_{\mathbb{Z}_{(p)}}\right)^{\circ}$ is the closure of $G^{\text {ado }}\left(\mathbb{Z}_{(p)}\right)^{+}$in $\mathscr{A}(G)^{\circ}$.

(2) $\mathscr{A}\left(G_{\mathbb{Z}_{(p)}}\right)^{\circ}$ is the completion of $G^{\text {ado }}\left(\mathbb{Z}_{(p)}\right)^{+}$with respect to the topology generated by images of sets of the form $G^{\mathrm{dero}}\left(\mathbb{Z}_{(p)}\right)+\cap \mathrm{K}^{p}$.

Proof. (1) is immediate from the definitions. Using 20] 2.0.13, one sees that $\mathscr{A}\left(G_{\mathbb{Z}_{(p)}}\right)^{\circ}$ is the completion of $G^{\text {ado }}\left(\mathbb{Z}_{(p)}\right)^{+}$with respect to the topology generated by images of sets of the form

$$
G^{\circ}\left(\mathbb{Z}_{(p)}\right)+\cap\left(\mathrm{K}^{p} \cap G^{\mathrm{der}}(\mathbb{Q})\right) \cdot U
$$

where $U$ is a finite index subgroup of the group of $p$-units in $Z_{G}(\mathbb{Q})$. Suppose $g=h u \in G^{\circ}\left(\mathbb{Z}_{(p)}\right)_{+}$with $h \in \mathrm{K}^{p} \cap G^{\mathrm{der}}(\mathbb{Q})$ and $u \in U$. Then $h$ fixes $x^{\text {ad }}$, so 
$h \in G^{\text {der }}\left(\mathbb{Z}_{(p)}\right)_{+}$. As in the proof of Lemma 4.3.5, this implies that $h \in G^{\text {dero }}\left(\mathbb{Z}_{(p)}\right)_{+}$, for $\mathrm{K}^{p}$ small enough. Thus for $\mathrm{K}^{p}$ small enough the image of the set above is equal to $\mathrm{K}^{p} \cap G^{\text {dero }}\left(\mathbb{Z}_{(p)}\right)_{+}$. This proves $(2)$.

4.6.5. Fix a connected component $X^{+} \subset X$. We denote by $\operatorname{Sh}(G, X)^{+} \subset \operatorname{Sh}(G, X)$ the connected Shimura variety corresponding to the choice of $X^{+}$, and similarly for $\operatorname{Sh}_{\mathrm{K}_{p}^{\circ}}(G, X)^{+} \subset \operatorname{Sh}_{\mathrm{K}_{p}^{\circ}}(G, X)$. Let $\mathrm{E}^{p} \subset \overline{\mathrm{E}}$ denote the maximal extension of $\mathrm{E}$ which is unramified at primes dividing $p$. By 4.3.2 and 20], Thm. 2.6.3, the action of $\operatorname{Gal}(\overline{\mathrm{E}} / \mathrm{E})$ on $\operatorname{Sh}_{K_{p}}(G, X)^{+}$factors through $\mathrm{Gal}\left(\mathrm{E}^{p} / \mathrm{E}\right)$. We again denote by $\operatorname{Sh}_{K_{p}^{\circ}}(G, X)^{+}$the $E^{p}$-scheme obtained from $\operatorname{Sh}_{K_{p}^{\circ}}(G, X)^{+}$by descent, and by $\mathscr{S}_{\mathrm{K}_{p}^{\circ}}(G, X)^{+} \subset \mathscr{S}_{\mathrm{K}_{p}^{\circ}}(G, X)$ the corresponding component of $\mathscr{S}_{\mathrm{K}_{p}^{\circ}}(G, X)$, which is defined over $\mathcal{O}_{\mathrm{E} p} \otimes_{\mathcal{O}} \mathcal{O}_{(v)}$.

Let $\mathscr{E}\left(G_{\mathbb{Z}_{(p)}}^{\circ}\right) \subset \mathscr{A}\left(G_{\mathbb{Z}_{(p)}}\right) \times \operatorname{Gal}\left(\mathrm{E}^{p} / \mathrm{E}\right)$ denote the stabilizer of $\operatorname{Sh}_{\mathrm{K}_{p}^{\circ}}(G, X)^{+} \subset$ $\operatorname{Sh}_{\mathrm{K}_{p}^{\circ}}(G, X)$ (viewed as $\mathrm{E}^{p}$-schemes), and let $\tilde{\mathscr{E}}\left(G_{\mathbb{Z}_{(p)}}^{\circ}\right) \subset \tilde{\mathscr{A}}\left(G_{\mathbb{Z}_{(p)}}\right) \times \operatorname{Gal}(\overline{\mathrm{E}} / \mathrm{E})$ denote the stabilizer of $\operatorname{Sh}(G, X)^{+} \subset \operatorname{Sh}(G, X)$.

Lemma 4.6.6. We have

(1) $\mathscr{E}\left(G_{\mathbb{Z}_{(p)}}^{\circ}\right)\left(\right.$ resp. $\left.\tilde{\mathscr{E}}\left(G_{\mathbb{Z}_{(p)}}^{\circ}\right)\right)$ is an extension of $\operatorname{Gal}\left(\mathrm{E}^{p} / \mathrm{E}\right)(\operatorname{resp} \cdot \operatorname{Gal}(\overline{\mathrm{E}} / \mathrm{E}))$ by $\mathscr{A}\left(G_{\mathbb{Z}_{(p)}}\right)^{\circ}\left(\right.$ resp. $\left.\tilde{\mathscr{A}}\left(G_{\mathbb{Z}_{(p)}}\right)^{\circ}\right)$.

(2) There are canonical isomorphisms

$$
\begin{aligned}
& \mathscr{A}\left(G_{\mathbb{Z}_{(p)}}\right) * \mathscr{A}\left(G_{\mathbb{Z}_{(p)}}\right) \circ \mathscr{E}\left(G_{\mathbb{Z}_{(p)}}^{\circ}\right) \cong \mathscr{A}\left(G_{\mathbb{Z}_{(p)}}\right) \times \operatorname{Gal}\left(\mathrm{E}^{p} / \mathrm{E}\right) \\
& \tilde{\mathscr{A}}\left(G_{\mathbb{Z}_{(p)}}\right) *{\tilde{\mathscr{A}}\left(G_{\mathbb{Z}_{(p)}}\right)}^{\circ} \tilde{\mathscr{E}}\left(G_{\mathbb{Z}_{(p)}}^{\circ}\right) \cong \tilde{\mathscr{A}}\left(G_{\mathbb{Z}_{(p)}}\right) \times \operatorname{Gal}(\overline{\mathrm{E}} / \mathrm{E}) .
\end{aligned}
$$

where an element of $\mathscr{E}\left(G_{\mathbb{Z}_{(p)}}^{\circ}\right)$ (resp. $\tilde{\mathscr{E}}\left(G_{\mathbb{Z}_{(p)}}^{\circ}\right)$ ) acts on $\mathscr{A}\left(G_{\mathbb{Z}_{(p)}}\right)$ (resp. $\left.\tilde{\mathscr{A}}\left(G_{\mathbb{Z}_{(p)}}\right)\right)$ via conjugation by its image in $\mathscr{A}\left(G_{\mathbb{Z}_{(p)}}\right)$.

Proof. Let $\mathrm{E}^{\times,+}=\mathrm{E}^{\times} \cap\left(\mathrm{E} \otimes_{\mathbb{Q}} \mathbb{R}\right)^{\times,+}$. Consider the composite map

$$
\mathbb{A}_{\mathrm{E}}^{\times} / \mathrm{E}^{\times}(\mathrm{E} \otimes \mathbb{Q} \mathbb{R})^{\times,+}=\mathbb{A}_{\mathrm{E}}^{f \times} / \mathrm{E}^{\times,+} \stackrel{\mu_{h}^{-1}}{\rightarrow} G\left(\mathbb{A}_{\mathrm{E}}^{f}\right) / G(\mathrm{E})_{+}^{-} \stackrel{N_{\mathrm{E} / \mathbb{Q}}}{\rightarrow} G\left(\mathbb{A}_{f}\right) / G(\mathbb{Q})_{+}^{-}
$$

where $G(\mathrm{E})_{+}^{-}=\left(R_{\mathrm{E} / \mathbb{Q}} G\right)(\mathbb{Q})_{+}^{-}$. If $x \in \mathbb{A}_{\mathrm{E}}^{f \times} / \mathrm{E}^{\times,+}$, then by weak approximation $x$ has a representative $\left(x_{v}\right) \in \mathbb{A}_{\mathrm{E}}^{f \times}$ with $x_{v} \in \mathcal{O}_{\mathrm{E}_{v}}^{\times}$for all $v \mid p$. Hence, by Lemma 4.3.2, the image of $x$ under (4.6.7) is contained in $G\left(\mathbb{A}_{f}^{p}\right) \times G_{\mathbb{Z}_{(p)}}^{\circ}\left(\mathbb{Z}_{p}\right) / G^{\circ}\left(\mathbb{Z}_{(p)}\right) \sim$. By [20], Thm. 2.6.3, the action of $\mathrm{Gal}(\overline{\mathrm{E}} / \mathrm{E})$ on the geometrically connected components of $\operatorname{Sh}(G, X)$ is given by the composite of (4.6.7) and the class field theory isomorphism. This proves the claim that $\tilde{\mathscr{E}}\left(G_{\mathbb{Z}_{(p)}}^{\circ}\right)$ is an extension of $\operatorname{Gal}(\overline{\mathrm{E}} / \mathrm{E})$ by $\tilde{\mathscr{A}}\left(G_{\mathbb{Z}_{(p)}}\right)^{\circ}$.

It follows that the action of $\mathrm{Gal}\left(\mathrm{E}^{p} / \mathrm{E}\right)$ on the geometrically connected components of $\operatorname{Sh}_{\mathrm{K}_{p}^{\circ}}(G, X)$ is given by the induced map

$$
\operatorname{Gal}\left(\mathrm{E}^{p} / \mathrm{E}\right) \rightarrow G\left(\mathbb{A}_{f}^{p}\right) \times G_{\mathbb{Z}_{(p)}}^{\circ}\left(\mathbb{Z}_{p}\right) / G^{\circ}\left(\mathbb{Z}_{(p)}\right)_{+}^{\sim} G_{\mathbb{Z}_{(p)}}\left(\mathbb{Z}_{p}\right) \cong G\left(\mathbb{A}_{f}^{p}\right) / G^{\circ}\left(\mathbb{Z}_{(p)}\right)_{+}^{-} .
$$

This shows that $\mathscr{E}\left(G_{\mathbb{Z}_{(p)}}^{\circ}\right)$ is an extension of $\operatorname{Gal}\left(\mathrm{E}^{p} / \mathrm{E}\right)$ by $\mathscr{A}\left(G_{\mathbb{Z}_{(p)}}\right)^{\circ}$. Now $(2)$ follows easily.

4.6.8. Let $G_{2}$ be a reductive group over $\mathbb{Q}$ equipped with a central isogeny $\alpha$ : $G^{\text {der }} \rightarrow G_{2}^{\text {der }}$. Let $x_{2} \in B\left(G_{2}, \mathbb{Q}_{p}\right)$ with $x_{2}^{\text {ad }}=x^{\text {ad }}$. We denote by $\mathcal{G}_{2}$ the model of $G_{2}$ defined as the stabilizer of $x_{2}$, and by $G_{2, \mathbb{Z}_{(p)}}$ and $G_{2, \mathbb{Z}_{(p)}}^{\circ}$ the group schemes over $\mathbb{Z}_{(p)}$ corresponding to $\mathcal{G}_{2}$ and $\mathcal{G}_{2}^{\circ}$. Write $K_{2, p}^{\circ}=\mathcal{G}_{2}^{\circ}\left(\mathbb{Z}_{p}\right)$. 
Suppose that we have a Shimura datum $\left(G_{2}, X_{2}\right)$ such that $\alpha$ induces an isomorphism of Shimura data

$$
\left(G^{\mathrm{ad}}, X^{\mathrm{ad}}\right) \cong\left(G_{2}^{\mathrm{ad}}, X_{2}^{\mathrm{ad}}\right) .
$$

By the real approximation theorem, after replacing $X_{2}$ by its conjugate by some element of $G_{2}^{\text {ad }}(\mathbb{Q})$, we may assume that the image of $X_{2} \subset X_{2}^{\text {ad }}$ contains $X^{+}$. We denote by $\mathrm{E}_{2}$ the reflex field $\left(G_{2}, X_{2}\right)$, and we set $\mathrm{E}^{\prime}=\mathrm{E} \cdot \mathrm{E}_{2}$. We denote by $\mathscr{E}_{\mathrm{E}^{\prime}}\left(G_{\mathbb{Z}_{(p)}}^{\circ}\right)$ and $\tilde{\mathscr{E}}_{\mathrm{E}^{\prime}}\left(G_{\mathbb{Z}_{(p)}}^{\circ}\right)$ the pullbacks of $\mathscr{E}\left(G_{\mathbb{Z}_{(p)}}^{\circ}\right)$ and $\tilde{\mathscr{E}}\left(G_{\mathbb{Z}_{(p)}}^{\circ}\right)$ by $\operatorname{Gal}\left(\mathrm{E}^{\prime p} / \mathrm{E}^{\prime}\right) \rightarrow \operatorname{Gal}\left(\mathrm{E}^{p} / \mathrm{E}\right)$ and $\operatorname{Gal}\left(\overline{\mathrm{E}} / \mathrm{E}^{\prime}\right) \rightarrow \operatorname{Gal}(\overline{\mathrm{E}} / \mathrm{E})$ respectively.

We have the groups $\mathscr{A}\left(G_{2}\right)$ and $\tilde{\mathscr{A}}\left(G_{2}\right)^{\circ}$ defined as above, and we set

$$
\mathscr{A}\left(G_{2, \mathbb{Z}_{(p)}}\right)=G_{2}\left(\mathbb{A}_{f}^{p}\right) / Z_{G_{2}}\left(\mathbb{Z}_{(p)}\right)^{-} *_{G_{2}^{\circ}\left(\mathbb{Z}_{(p)}\right)_{+} / Z_{G_{2}}\left(\mathbb{Z}_{(p)}\right)} G^{\text {ado }}\left(\mathbb{Z}_{(p)}\right)^{+},
$$

and similarly for $\tilde{\mathscr{A}}\left(G_{2, \mathbb{Z}_{(p)}}\right)^{\circ}$. Note that the group $G^{\text {ado }}\left(\mathbb{Z}_{(p)}\right)^{+}$is exactly the same one which appeared in the definition of $\mathscr{A}\left(G_{\mathbb{Z}_{(p)}}\right)$.

As in Corollary 4.3.9, the geometrically connected components of $\operatorname{Sh}_{\kappa_{2, p}^{\circ}}\left(G_{2}, X_{2}\right)$ are defined over $\mathrm{E}_{2}^{p}$. We define $\mathscr{E}\left(G_{2, \mathbb{Z}_{(p)}}^{\circ}\right) \subset \mathscr{A}\left(G_{2, \mathbb{Z}_{(p)}}\right) \times \operatorname{Gal}\left(\mathrm{E}_{2}^{p} / \mathrm{E}_{2}\right)$ as the stabilizer of $\operatorname{Sh}_{\mathrm{K}_{2, p}^{\circ}}\left(G_{2}, X_{2}\right)^{+} \subset \operatorname{Sh}_{\mathrm{K}_{2, p}^{\circ}}\left(G_{2}, X_{2}\right)$. As in the proof of, Lemma 4.6.6, this is an extension of $\operatorname{Gal}\left(\mathrm{E}_{2}^{p} / \mathrm{E}_{2}\right)$ by $\mathscr{A}\left(G_{2, \mathbb{Z}_{(p)}}\right)^{\circ}$.

Similarly, we define $\tilde{\mathscr{E}}\left(G_{2, \mathbb{Z}_{(p)}}^{\circ}\right)$ as above. It is an extension of $\mathrm{Gal}\left(\overline{\mathrm{E}} / \mathrm{E}_{2}\right)$ by $\tilde{\mathscr{A}}\left(G_{2, \mathbb{Z}_{(p)}}\right)^{\circ}$.

Lemma 4.6.9. There exist natural maps of extensions

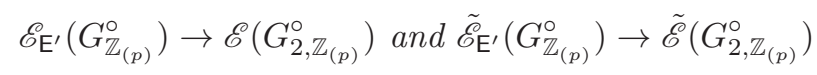

Proof. (cf. 20, 2.5.6) Let $G_{3}$ be the connected component of the identity of $G \times{ }_{G^{\text {ad }}}$ $G_{2}$, and $X_{3}$ the conjugacy class of homomorphisms $\mathbb{S} \rightarrow G_{3, \mathbb{R}}$ induced by $X$ and $X_{2}$. Repeating the above definitions for $G_{3}$ we obtain an extension $\mathscr{E}\left(G_{3, \mathbb{Z}_{(p)}}^{\circ}\right)$. Note that the reflex field of $\left(G_{3}, X_{3}\right)$ is $\mathrm{E}^{\prime}$, and $G_{3}^{\text {der }}=G^{\text {der }}$. Thus $G_{3}$ satisfies the condition (4.3.4), as we are assuming $G$ does. In particular, we have $\mathscr{A}\left(G_{3, \mathbb{Z}_{(p)}}\right)^{\circ} \cong \mathscr{A}\left(G_{\mathbb{Z}_{(p)}}\right)^{\circ}$ by Lemma $4.6 .4(2)$. It follows that the natural map $\mathscr{E}\left(G_{3, \mathbb{Z}_{(p)}}^{\circ}\right) \rightarrow \mathscr{E}_{E^{\prime}}\left(G_{\mathbb{Z}_{(p)}}^{\circ}\right)$ is an isomorphism of extensions. The first map of the lemma is given by the composite

$$
\mathscr{E}_{\mathrm{E}^{\prime}}\left(G_{\mathbb{Z}_{(p)}}^{\circ}\right) \cong \mathscr{E}\left(G_{3, \mathbb{Z}_{(p)}}^{\circ}\right) \rightarrow \mathscr{E}\left(G_{2, \mathbb{Z}_{(p)}}^{\circ}\right) .
$$

The construction for the second map is analogous.

Lemma 4.6.10. The diagram

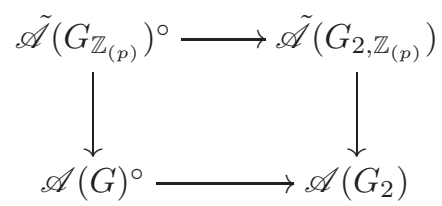

is commutative and Cartesian. In particular, the morphism of complexes

$$
\left(\tilde{\mathscr{A}}\left(G_{\mathbb{Z}_{(p)}}\right)^{\circ} \rightarrow \tilde{\mathscr{A}}\left(G_{2, \mathbb{Z}_{(p)}}\right)\right) \rightarrow\left(\mathscr{A}(G)^{\circ} \rightarrow \mathscr{A}\left(G_{2}\right)\right) .
$$

induces a bijection on kernels and an injection on cokernels. 
Proof. (cf. 43] Lemma 3.3.3.) We remark that the top map is well defined by Lemma 4.6.4(1). The diagram commutes, since $G^{\text {ado }}\left(\mathbb{Z}_{(p)}\right)^{+}$is naturally a subgroup of each term, is dense in $\left(\tilde{\mathscr{A}}\left(G_{\mathbb{Z}_{(p)}}\right)^{\circ}\right.$, and all the maps are the identity on $G^{\text {ado }}\left(\mathbb{Z}_{(p)}\right)^{+}$.

Suppose that $(g, \gamma) \in \tilde{\mathscr{A}}\left(G_{2, \mathbb{Z}_{(p)}}\right)$ is in the image of $\left(g_{1}, \gamma_{1}\right) \in \mathscr{A}(G)^{\circ}$. Since $g_{1}$ may be approximated by an element of $G(\mathbb{Q})_{+}$, we may assume that $g_{1}$ is in the image of $G\left(\mathbb{A}_{f}^{p}\right) \times G^{\circ}\left(\mathbb{Z}_{p}\right)$. Since $g \gamma=g_{1} \gamma_{1}$ in $G^{\text {ad }}\left(\mathbb{A}_{f}\right)$, we have $\gamma_{1}=g_{1}^{-1} g \gamma \in$ $G^{\text {ado }}\left(\mathbb{Z}_{(p)}\right)_{+}$so $\left(g_{1}, \gamma_{1}\right) \in \tilde{\mathscr{A}}\left(G_{\mathbb{Z}_{(p)}}\right)^{\circ}$. Thus the diagram in the lemma is Cartesian.

4.6.12. By Lemma 4.6.10 we have an inclusion

$$
\mathscr{A}\left(G_{\mathbb{Z}_{(p)}}\right)^{\circ} \backslash \mathscr{A}\left(G_{2, \mathbb{Z}_{(p)}}\right)=\tilde{\mathscr{A}}\left(G_{\left.\mathbb{Z}_{(p)}\right)}\right)^{\circ} \backslash \tilde{\mathscr{A}}\left(G_{2, \mathbb{Z}_{(p)}}\right) / \mathrm{K}_{2, p}^{\circ} \hookrightarrow \mathscr{A}(G)^{\circ} \backslash \mathscr{A}\left(G_{2}\right) / \mathrm{K}_{2, p}^{\circ} .
$$

Let $J \subset G_{2}\left(\mathbb{Q}_{p}\right)$ denote a set which maps bijectively to a set of coset representatives for the image of $\mathscr{A}\left(G_{2, \mathbb{Z}_{(p)}}\right)$ in $\mathscr{A}(G)^{\circ} \backslash \mathscr{A}\left(G_{2}\right) / \mathrm{K}_{2, p}^{\circ}$.

Recall, we assume either that the center $Z$ of $G$ is connected or that $Z_{G^{\text {der }}}$ has rank prime to $p$.

Lemma 4.6.13. There is an isomorphism of $\overline{\mathrm{E}}$-schemes with $G_{2}\left(\mathbb{A}_{f}^{p}\right) \times \operatorname{Gal}\left(\overline{\mathrm{E}} / \mathrm{E}^{\prime}\right)$ action

$$
\operatorname{Sh}_{\mathrm{K}_{2, p}^{\circ}}\left(G_{2}, X_{2}\right) \cong\left[\left[\operatorname{Sh}_{\mathrm{K}_{p}^{\circ}}(G, X)^{+} \times \mathscr{A}\left(G_{2, \mathbb{Z}_{(p)}}\right)\right] / \mathscr{A}\left(G_{\mathbb{Z}_{(p)}}\right)^{\circ}\right]^{|J|}
$$

where $h \in \mathscr{A}\left(G_{\mathbb{Z}_{(p)}}\right)^{\circ}$ acts on $\mathscr{A}\left(G_{\mathbb{Z}_{(p)}}\right)$ by left multiplication by $h^{-1}$.

Proof. By [20] 2.5.6, there is a morphism of extensions $\mathscr{E}_{\mathrm{E}^{\prime}}(G) \rightarrow \mathscr{E}\left(G_{2}\right)$, and in particular, an isomorphism

$$
\tilde{\mathscr{A}}\left(G_{2}\right) * \tilde{\mathscr{A}}(G)^{\circ} \mathscr{E}_{\mathrm{E}^{\prime}}(G) \cong G_{2}\left(\mathbb{A}_{f}\right) \times \operatorname{Gal}\left(\overline{\mathrm{E}} / \mathrm{E}^{\prime}\right) .
$$

We equip $\operatorname{Sh}(G, X)^{+} \times \mathscr{A}\left(G_{2}\right)$ with a right action of $\mathscr{E}_{\mathrm{E}^{\prime}}(G)$ given by $(s, a) \cdot e=$ $\left(s e, \bar{e}^{-1} a \bar{e}\right)$, and with the action of $\mathscr{A}\left(G_{2}\right)$ induced by right multiplication of $\mathscr{A}\left(G_{2}\right)$ on itself. Here $\bar{e}$ denotes the image of $e$ under

$$
\mathscr{E}_{\mathrm{E}^{\prime}}(G) \rightarrow \mathscr{E}\left(G_{2}\right) \rightarrow \mathscr{A}\left(G_{2}\right) .
$$

This induces an action of $\mathscr{A}\left(G_{2}\right) \rtimes \mathscr{E}_{\mathrm{E}^{\prime}}(G)$ on $\operatorname{Sh}(G, X)^{+} \times \mathscr{A}\left(G_{2}\right)$, which descends to an action of $\mathscr{A}\left(G_{2}\right) * \mathscr{A}(G)^{\circ} \mathscr{E}_{E^{\prime}}(G)$ on $\left[\operatorname{Sh}(G, X)^{+} \times \mathscr{A}\left(G_{2}\right)\right] / \mathscr{A}(G)^{\circ}$.

By 20 2.7.11, 2.7.13, using the above isomorphism gives an isomorphism of $\overline{\mathrm{E}}$-schemes with $G_{2}\left(\mathbb{A}_{f}\right) \times \operatorname{Gal}\left(\overline{\mathrm{E}} / \mathrm{E}^{\prime}\right)$-action

$$
\operatorname{Sh}\left(G_{2}, X_{2}\right) \cong\left[\operatorname{Sh}(G, X)^{+} \times \mathscr{A}\left(G_{2}\right)\right] / \mathscr{A}(G)^{\circ} .
$$

Dividing both sides by $\mathrm{K}_{2, p}^{\circ}$ we obtain an isomorphism of $\overline{\mathrm{E}}$-schemes with $G_{2}\left(\mathbb{A}_{f}^{p}\right) \times$ $\operatorname{Gal}\left(\overline{\mathrm{E}} / \mathrm{E}^{\prime}\right)$-action

$$
\begin{aligned}
\operatorname{Sh}_{\mathrm{K}_{2, p}^{\circ}}\left(G_{2}, X_{2}\right) \cong\left[\operatorname{Sh}(G, X)^{+} \times \mathscr{A}\left(G_{2}\right) / \mathrm{K}_{2, p}^{\circ}\right] / \mathscr{A}(G)^{\circ} \\
\cong \coprod_{j}\left[\operatorname{Sh}(G, X)^{+} \times \mathscr{A}\left(G_{2, \mathbb{Z}_{(p)}}\right) j\right] / \tilde{\mathscr{A}}\left(G_{\mathbb{Z}_{(p)}}\right)^{\circ} .
\end{aligned}
$$

Since $\mathrm{K}_{p}^{\circ} \cap \tilde{\mathscr{A}}\left(G_{\mathbb{Z}_{(p)}}\right)^{\circ}$ is contained in the kernel of the composite

$$
\tilde{\mathscr{A}}\left(G_{\mathbb{Z}_{(p)}}\right)^{\circ} \rightarrow \mathscr{A}\left(G_{\mathbb{Z}_{(p)}}\right)^{\circ} \rightarrow \mathscr{A}\left(G_{\mathbb{Z}_{2,(p)}}\right)^{\circ},
$$


the final quotient above is equal to

$$
\coprod_{j \in J}\left[\operatorname{Sh}_{K_{p}^{\circ}}(G, X)^{+} \times \mathscr{A}\left(G_{2, \mathbb{Z}_{(p)}}\right) j\right] / \mathscr{A}\left(G_{\mathbb{Z}_{(p)}}\right)^{\circ} .
$$

The lemma follows.

Corollary 4.6.15. The $\mathcal{O}_{\mathrm{E}^{\prime p},(v)}=\mathcal{O}_{\mathrm{E}^{\prime p}} \otimes_{\mathcal{O}} \mathcal{O}_{(v)}$-scheme

$$
\mathscr{S}_{\mathrm{K}_{2, p}^{\circ}}\left(G_{2}, X_{2}\right)=\left[\left[\mathscr{S}_{\mathrm{K}_{p}^{\circ}}(G, X)^{+} \times \mathscr{A}\left(G_{2, \mathbb{Z}_{(p)}}\right)\right] / \mathscr{A}\left(G_{\mathbb{Z}_{(p)}}\right)^{\circ}\right]^{|J|}
$$

has a natural structure of a $\mathcal{O}_{(v)}^{\prime}=\mathcal{O}_{\mathrm{E}^{\prime}} \otimes \mathcal{O}_{\mathcal{O}} \mathcal{O}_{(v)}$-scheme with $G_{2}\left(\mathbb{A}_{f}^{p}\right)$-action, and is a model for $\operatorname{Sh}_{\mathrm{K}_{2, p}^{\circ}}\left(G_{2}, X_{2}\right)$. The local rings on $\mathscr{S}_{\mathrm{K}_{2, p}^{\circ}}\left(G_{2}, X_{2}\right) \otimes_{\mathcal{O}} \mathcal{O}_{v}$ are étale locally isomorphic to those on $\mathrm{M}_{G, X}^{\mathrm{loc}} \otimes_{\mathcal{O}_{v}} \mathcal{O}_{v}^{\prime}$.

Proof. As observed in 4.6.3, the action of $\mathscr{A}\left(G_{\mathbb{Z}_{(p)}}\right)$ on $\operatorname{Sh}_{\mathrm{K}_{p}^{\circ}}(G, X)$ extends to

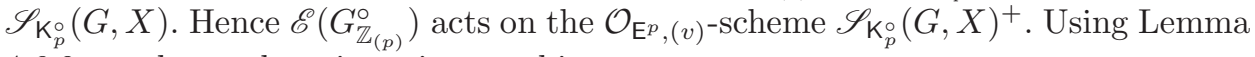
4.6.9, as above, there is an isomorphism

$$
\left.\mathscr{A}\left(G_{\left.2, \mathbb{Z}_{(p)}\right)}\right) \mathscr{A}_{\left(G_{\mathbb{Z}_{(p)}}\right.}\right)^{\circ} \mathscr{E}_{\mathrm{E}^{\prime}}\left(G_{\mathbb{Z}_{(p)}}^{\circ}\right) \cong G_{2}\left(\mathbb{A}_{f}^{p}\right) \times \operatorname{Gal}\left(\mathrm{E}^{\prime p} / \mathrm{E}^{\prime}\right) .
$$

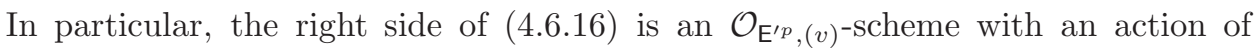
$G_{2}\left(\mathbb{A}_{f}^{p}\right) \times \operatorname{Gal}\left(\mathrm{E}^{\prime p} / \mathrm{E}^{\prime}\right)$. Hence by Galois descent it is naturally an $\mathcal{O}_{(v)}^{\prime}$-scheme with an action of $G_{2}\left(\mathbb{A}_{f}^{p}\right)$. The first statement is now a consequence of Lemma 4.6.13.

The second statement then follows from Theorem 4.2.7 and Proposition 4.3.7 once we show that

$$
\Delta\left(G, G_{2}\right):=\operatorname{ker}\left(\mathscr{A}\left(G_{\mathbb{Z}_{(p)}}\right)^{\circ} \rightarrow \mathscr{A}\left(G_{2, \mathbb{Z}_{(p)}}\right)\right)
$$

acts freely on $\mathscr{S}_{\mathrm{K}_{p}}(G, X)^{+}$. For this we follow the proof of [43] Prop. 3.4.6, which can be modified to work in our present setting because we have defined the twisting construction $\mathscr{A} \mapsto \mathscr{A}^{\mathcal{P}}$ on the level of abelian varieties and not just in the isogeny category.

Let $\left(h, \gamma^{-1}\right) \in \Delta\left(G, G_{2}\right)$ with $h \in G\left(\mathbb{A}_{f}^{p}\right)$ and $\gamma \in G^{\text {ado }}\left(\mathbb{Z}_{(p)}\right)^{+}$. Denote by $\mathcal{P}$ the $Z$-torsor associated to $\gamma$, and fix a Galois extension $F / \mathbb{Q}$ and a point $\tilde{\gamma} \in \mathcal{P}\left(\mathcal{O}_{F,(p)}\right)$ lifting $\gamma$.

Let $x \in \mathscr{S}_{\mathrm{K}_{p}^{\circ}}(G, X)(T)$, where $T$ is the spectrum of an algebraically closed field, and suppose that $\left(h, \gamma^{-1}\right)$ fixes $x$. Write $\left(\mathcal{A}_{x}, \lambda, \varepsilon^{p}\right)$ for the corresponding triple. Then by Lemma 4.5.7, for every compact open subgroup $\mathrm{K}^{p} \subset G\left(\mathbb{A}_{f}^{p}\right)$ there exists

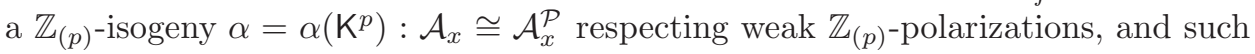
that the left hand square of the following diagram commutes modulo $\mathrm{K}^{p}$ (That is up to multiplication by an element of $\mathrm{K}^{p}$ on the bottom left hand corner.)

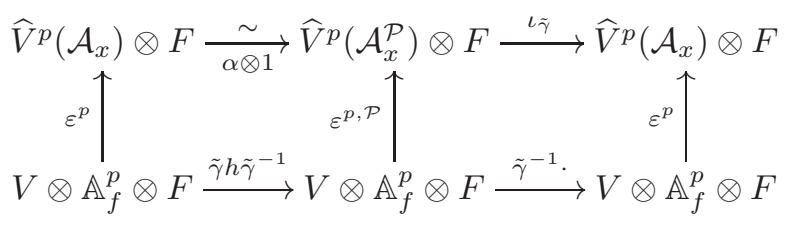

while the right square commutes by the definition of $\varepsilon^{p, \mathcal{P}}$.

For $\mathrm{K}^{p}$ sufficiently small, the map $\alpha\left(\mathrm{K}^{p}\right)$ is unique. Hence if $\mathrm{K}^{p}$ is sufficiently small then $\alpha$ does not depend on $\mathrm{K}^{p}$, and we may assume that 4.6 .17 commutes. 
Note that the composite of the maps in the lower row of 4.6 .17 is $h \tilde{\gamma}^{-1}$. Since $\left(h, \gamma^{-1}\right) \in \Delta\left(G, G_{2}\right)$, we have $h \tilde{\gamma}^{-1} \in Z\left(\mathbb{A}_{f}^{p} \otimes F\right)$, so

$$
\iota_{\tilde{\gamma}} \circ \alpha \in Z\left(\mathbb{A}_{f}^{p} \otimes F\right) \cap\left(\underline{\mathrm{Aut}}_{\mathbb{Z}_{(p)}} \mathcal{A}_{x}\right)\left(\mathcal{O}_{F,(p)}\right)=Z\left(\mathcal{O}_{F,(p)}\right) \subset\left(\underline{\mathrm{Aut}}_{\mathbb{Q}} \mathcal{A}_{x}\right)\left(\mathbb{A}_{f}^{p} \otimes F\right) .
$$

Hence $h \tilde{\gamma}^{-1} \in Z\left(\mathcal{O}_{F,(p)}\right)$, and after replacing $\tilde{\gamma}$ with another lift, we may assume that $h \tilde{\gamma}^{-1}=1$. Then $\tilde{\gamma}$ is $\operatorname{Gal}(F / \mathbb{Q})$ invariant, so $\tilde{\gamma} \in G\left(\mathbb{Z}_{(p)}\right)_{+}$.

In this case the action of $\left(h, \gamma^{-1}\right)$ on $\operatorname{Sh}_{\mathrm{K}_{p}^{\circ}}(G, X)$ is by the natural right action of $h \tilde{\gamma}^{-1} \in G\left(\mathbb{A}_{f}\right)$, which is given by the action of $\tilde{\gamma}^{-1} \in G\left(\mathbb{Q}_{p}\right)$, since $h \tilde{\gamma}^{-1}=1$ in $G\left(\mathbb{A}_{f}^{p}\right)$. It follows from Lemma 4.3.5 that $\tilde{\gamma}^{-1} \in G^{\circ}\left(\mathbb{Z}_{(p)}\right)$, so $\left(h, \gamma^{-1}\right)=1$.

Corollary 4.6.18. Extend $v$ to an embedding $v^{\prime}: \mathrm{E}^{\prime} \hookrightarrow E^{\mathrm{ur}}$, and set $E^{\prime}=\mathrm{E}_{v^{\prime}}^{\prime}$. We equip $\mathrm{M}_{G, X}^{\mathrm{loc}}$ with the trivial $G_{2}\left(\mathbb{A}_{f}^{p}\right)$-action. There is a diagram of $\mathcal{O}_{E^{\prime}}$-schemes with $G_{2}\left(\mathbb{A}_{f}^{p}\right)$-action

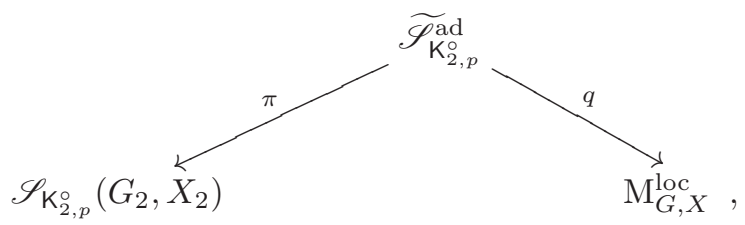

where $\pi$ is a $G_{\mathbb{Z}_{p}}^{\mathrm{ad}}$-torsor, and $q$ is $G_{\mathbb{Z}_{p}}^{\mathrm{ad}}$-equivariant.

Moreover, any sufficiently small compact open $\mathrm{K}_{2}^{p} \subset G_{2}\left(\mathbb{A}_{f}^{p}\right)$ acts freely on $\widetilde{\mathscr{S}_{\mathrm{K}_{2, p}^{\circ}}^{\mathrm{d}}}$, and the morphism $\widetilde{\mathscr{S}_{\mathrm{K}_{2, p}^{\circ}}^{\mathrm{ad}}} / \mathrm{K}_{2}^{p} \rightarrow \mathrm{M}_{G, X}^{\mathrm{loc}}$, induced by $q$, is smooth of relative dimension $\operatorname{dim} G^{\mathrm{ad}}$.

Proof. By Lemma 4.6.2 the $\mathscr{B}\left(G_{\mathbb{Z}_{(p)}}\right)$-action on the $G_{\mathbb{Z}_{p}}^{\text {ad }}$-torsor $\widetilde{\mathscr{S}}_{\mathrm{K}_{p}}^{\text {ad }}$ given by Lemma 4.5.9, restricts to an $\mathscr{A}\left(G_{\mathbb{Z}_{(p)}}\right)$-action. Let $\widetilde{\mathscr{S}}_{\mathrm{K}_{p}^{\circ}}^{\circ}+$ denote the pullback of $\widetilde{\mathscr{S}}_{\mathrm{K}_{p}^{\text {ad }}}$ to $\mathscr{S}_{\mathrm{K}_{p}^{\circ}}(G, X)_{\mathcal{O}_{E \text { ur }}}^{+}$. Denote by $\mathscr{E}_{E^{\prime \prime}}\left(G_{\mathbb{Z}_{(p)}}^{\circ}\right)$ the pullback of $\mathscr{E}_{\mathrm{E}^{\prime}}\left(G_{\mathbb{Z}_{(p)}}^{\circ}\right)$ by $\operatorname{Gal}\left(E^{\mathrm{ur}} / E^{\prime}\right) \rightarrow \operatorname{Gal}\left(\mathrm{E}^{p} / \mathrm{E}^{\prime}\right)$. Then the stabilizer of $\widetilde{\mathscr{S}_{\mathrm{K}_{p}^{\circ}}^{\mathrm{ad}}+}$ in $\mathscr{A}\left(G_{\mathbb{Z}_{(p)}}\right) \times \operatorname{Gal}\left(E^{\mathrm{ur}} / E^{\prime}\right)$ is $\mathscr{E}_{E^{\prime \prime}}\left(G_{\mathbb{Z}_{(p)}}^{\circ}\right)$. Set

$$
\widetilde{\mathscr{S}}_{\mathrm{K}_{2, p}^{\circ}}^{\mathrm{ad}}=\left[\left[\widetilde{\mathscr{S}}_{\mathrm{K}_{p}^{\circ}}^{\mathrm{ad}}+\times \mathscr{A}\left(G_{2, \mathbb{Z}_{(p)}}\right)\right] / \mathscr{A}\left(G_{\mathbb{Z}_{(p)}}\right)^{\circ}\right]^{|J|}
$$

Note that the group $\operatorname{ker}\left(\mathscr{A}\left(G_{\mathbb{Z}_{(p)}}\right)^{\circ} \rightarrow \mathscr{A}\left(G_{2, \mathbb{Z}_{(p)}}\right)\right)$ need not be finite, but this causes no difficulty in the formation of the quotient; see [44, E.7].

As above, $\widetilde{\mathscr{S}_{\mathrm{K}_{2, p}^{\circ}}^{\mathrm{ad}}}$ is equipped with an action of $G_{2}\left(\mathbb{A}_{f}^{p}\right) \times \operatorname{Gal}\left(E^{\mathrm{ur}} / E^{\prime}\right)$, and de-

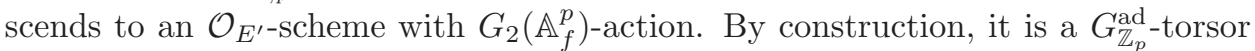
over $\mathscr{S}_{\mathrm{K}_{2, p}^{\circ}}\left(G_{2}, X_{2}\right)$, and $q$ is $G_{\mathbb{Z}_{p}}^{\text {ad }}$-equivariant. The final claim can be checked over $\mathcal{O}_{E^{\text {ur }}}$, when it follows easily from Lemma 4.5.9

4.6.21. Let $(H, Y)$ be a Shimura datum with $H^{\text {ad }}$ a classical group. Recall (20], cf. 43 3.4.13) that there is central isogeny $\tilde{H} \rightarrow H^{\sharp}$ (which depends also on $Y$ if $H$ has a factor of type $D)$ such that $(H, Y)$ is of abelian type if and only if $H^{\text {der }}$ is a quotient of $H^{\sharp}$.

For the remainder of this subsection we let $\left(G_{2}, X_{2}\right)$ be a Shimura datum of abelian type with reflex field $\mathrm{E}_{2}$. We choose $x_{2} \in \mathcal{B}\left(G_{2}, \mathbb{Q}_{p}\right)$, and we denote by $\mathrm{K}_{2, p}^{\circ} \subset G_{2}\left(\mathbb{Q}_{p}\right)$ the corresponding compact open, parahoric subgroup and by $\mathrm{K}_{2, p} \subset$ $G_{2}\left(\mathbb{Q}_{p}\right)$ the stabilizer of $x_{2}$. As always we assume $p>2$. 
Lemma 4.6.22. Suppose that $G_{2}$ splits over a tamely ramified extension of $\mathbb{Q}_{p}$. Then there exists a Shimura datum of Hodge type $(G, X)$, together with a central isogeny $G^{\text {der }} \rightarrow G_{2}^{\text {der }}$, which induces an isomorphism $\left(G^{\text {ad }}, X^{\text {ad }}\right) \cong\left(G_{2}^{\text {ad }}, X_{2}^{\text {add }}\right)$. Moreover, $(G, X)$ can be chosen to satisfy the following conditions.

(1) $\pi_{1}\left(G^{\mathrm{der}}\right)$ is a 2-group, and is trivial if $\left(G^{\mathrm{ad}}, X^{\mathrm{ad}}\right)$ has no factors of type $D^{\mathbb{H}}$. Moreover $G$ satisfies the condition (4.3.4).

(2) $G$ splits over a tamely ramified extension of $\mathbb{Q}_{p}$.

(3) If $\mathrm{E}$ denote the reflex field of $(G, X)$ and $\mathrm{E}^{\prime}=\mathrm{E} \cdot \mathrm{E}_{2}$, then any primes $v_{2} \mid p$ of $\mathrm{E}_{2}$ splits completely in $\mathrm{E}^{\prime}$.

(4) $Z_{G}$ is a torus.

(5) $\mathbb{X}_{\bullet}\left(G^{\mathrm{ab}}\right)_{I_{\mathbb{Q}_{p}}}$ is torsion free, where $I_{\mathbb{Q}_{p}} \subset G_{\mathbb{Q}_{p}}$ denotes the inertia subgroup.

Proof. Write $G_{2}^{\text {ad }}=\prod_{i} G_{i}$ where each $G_{i}$ is $\mathbb{Q}$-simple and adjoint. Then $G_{i}=$ $\operatorname{Res}_{F_{i} / \mathbb{Q}} G_{i}^{\prime}$ where $F_{i}$ is totally real, and $G_{i}^{\prime}$ is absolutely simple over $F_{i}$ and adjoint. Since we are assuming that $G$ splits over a tamely ramified extension of $\mathbb{Q}_{p}$ each $F_{i}$ is tamely ramified over $p$.

Choose $(G, X)$ of Hodge type such that there exists a central isogeny $G^{\text {der }} \rightarrow G_{2}^{\text {der }}$ inducing an isomorphism of Shimura data $\left(G^{\text {ad }}, X^{\text {ad }}\right) \cong\left(G_{2}^{\text {ad }}, X_{2}^{\text {ad }}\right)$. By 20 2.3.10, we may choose $G$ so that $G^{\text {der }}=\prod \operatorname{Res}_{F_{i} / \mathbb{Q}} G_{i}^{\prime \sharp}$. Then $\pi_{1}\left(G^{\text {der }}\right)$ is an elementary 2group (the only contributions comes from factors $G_{i}^{\prime}$ of type $\left.D^{\mathbb{H}}\right)$, and in particular $p \nmid \mid \pi_{1}\left(G^{\text {der }}\right)$. Moreover, $\operatorname{ker}\left(\tilde{G}^{\text {der }} \rightarrow G^{\text {der }}\right)$ has the form $\prod \operatorname{Res}_{F_{i} / \mathbb{Q}} C_{i}$ where each $C_{i}$ is either trivial, or $\mu_{2}$. In particular one sees using Cebotarev density that the condition (4.3.4) is satisfied.

Next we explain how to choose $(G, X)$ so that $G$ splits over a tamely ramified extension of $\mathbb{Q}_{p}$, and any prime $v \mid p$ of $\mathrm{E}^{\text {ad }}=E\left(G^{\text {ad }}, X^{\text {ad }}\right)$ splits completely in $\mathrm{E}=E(G, X)$. Suppose first that $G^{\text {ad }}=\operatorname{Res}_{F / \mathbb{Q}} G^{\prime}$. Following 20] 2.3, let $I_{c}$ be the set of real places $v$ of $F$ such that $G^{\prime}\left(F_{v}\right)$ is compact, and $I_{n c}$ the real places of $F$ not in $I_{c}$. Let $K / F$ be a quadratic, totally imaginary extension of $F$ in which the primes above $p$ split completely. Fix an isomorphism $\mathbb{C} \cong \overline{\mathbb{Q}}_{p}$. Then $\operatorname{Gal}\left(\overline{\mathbb{Q}}_{p} / \mathbb{Q}_{p}\right)$ acts on the embeddings $K \hookrightarrow \mathbb{C}$. Let $T$ be a set of embeddings $K \hookrightarrow \mathbb{C}$ which map bijectively to $I_{c}$, and such that if $\tau \in \operatorname{Gal}\left(\overline{\mathbb{Q}}_{p} / \mathbb{Q}_{p}\right)$ preserves $I_{c}$ then it preserves $T$. This is possible since all the primes of $F$ above $p$ split completely in $K$.

Define a morphism $h_{T}: \mathbb{C}^{\times} \rightarrow K \otimes_{\mathbb{Q}} \mathbb{R}$ by requiring that $K \otimes_{\sigma} \mathbb{C}$ has type $(-1,0)$ if $\sigma \in T$, type $(0,-1)$ if $\bar{\sigma} \in T$, and type $(0,0)$ otherwise. If $\tau \in \operatorname{Gal}\left(\overline{\mathbb{Q}}_{p} / \mathbb{Q}_{p}\right)$ fixes $\mathrm{E}^{\text {ad }}$, then in particular it preserves $I_{c}$, and hence preserves $T$, and fixes $E\left(K^{\times}, h_{T}\right)$. This implies that any prime of $\mathrm{E}^{\text {ad }}$ above $p$ splits completely in $\mathrm{E}^{\text {ad }} \cdot E\left(K^{\times}, h_{T}\right)$. By 20] $2.3 .10,(G, X)$ may be chosen so that $\mathrm{E}=\mathrm{E}^{\mathrm{ad}} \cdot E\left(K^{\times}, h_{T}\right)$. In particular, any prime of $\mathrm{E}^{\text {ad }}$ over $p$ splits completely in $\mathrm{E}$. Moreover, the construction of loc. cit produces a group such that $Z_{G}$ is contained in a product of $Z_{G^{\text {der }}}, K^{\times}$, and a torus which splits over the fixed field of the subgroup of $\operatorname{Gal}(\overline{\mathbb{Q}} / \mathbb{Q})$ which acts trivially on the Dynkin diagram of $G^{\text {ad }}$. In particular $G$ splits over a tamely ramified extension of $\mathbb{Q}_{p}$. In general, when $G^{\text {ad }}$ is not assumed simple, the above construction applies to each of the factors $\operatorname{Res}_{F_{i} / \mathbb{Q}} G_{i}^{\prime}$. Finally, any prime $v_{2} \mid p$ of $\mathrm{E}_{2}$ splits completely in $\mathrm{E}^{\prime}=\mathrm{E} \cdot \mathrm{E}_{2}$.

We will now show that we can arrange so that, in addition, the center $Z$ of $G$ is connected. Let $(G, X) \hookrightarrow\left(\operatorname{GSp}(V), S^{ \pm}\right)$be the Hodge embedding. Choose $h \in X$ corresponding to a special point; there is a maximal torus $T_{0}$ in $G$ defined over $\mathbb{Q}$, such that $h$ factors through $T_{0 \mathbb{R}}$. By an argument as in 43 , proof of Prop. 2.2.4, one 
sees that $h$ and $T_{0}$ can be chosen so that $T_{0}$ splits over a tamely ramified extension of $\mathbb{Q}_{p}$. Observe that $T_{0 \mathbb{R}} / w_{h}\left(\mathbb{G}_{m}\right)$ is compact, as $T_{0 \mathbb{R}} / w_{h}\left(\mathbb{G}_{m}\right) \hookrightarrow \operatorname{GSp}\left(V_{\mathbb{R}}\right) / \operatorname{diag}\left(\mathbb{G}_{m}\right)$ and the centralizer of $h$ in $\operatorname{GSp}\left(V_{\mathbb{R}}\right) / \operatorname{diag}\left(\mathbb{G}_{m}\right)$ is compact. Consider $G^{\prime}=(G \times$ $\left.T_{0}\right) / Z_{G}$. Then the center of $G^{\prime}$ is $T_{0}$ (which is connected), and $G, G^{\prime}$ have the same derived group.

Let $W=\operatorname{Hom}_{Z_{G}}(V, V)$ (QQQ-linear maps which are $Z_{G}$-equivariant). The group $G^{\prime}$ acts on $W$ via $((g, t) \cdot f)(x)=g f\left(t^{-1} x\right)$. Since $W$ contains $G$, one sees easily see that this $G^{\prime}$-action is faithful. We equip $W$ with a Hodge structure by writing $W=\operatorname{Hom}_{Z}\left(V_{2}, V\right)$, where $V_{2}$ is $V$ with trivial Hodge structure; the corresponding Deligne cocharacter $h^{\prime}$ of $G^{\prime}$ is given by $h \times 1$. Then $W$ has type $\{(-1,0),(0,-1)\}$. Since $T_{0 \mathbb{R}} / w_{h}\left(\mathbb{G}_{m}\right)$ is compact, it follows that ad $h^{\prime}(i)$ gives a Cartan involution on $G_{\mathbb{R}}^{\prime} / w_{h^{\prime}}\left(\mathbb{G}_{m}\right)$. Hence, we can apply [20] Prop. 2.3.2, to obtain an alternating form on $W$ and a corresponding Hodge embedding $\left(G^{\prime}, X^{\prime}\right) \hookrightarrow\left(\mathrm{GSp}(W), S_{W}^{ \pm}\right)$. Notice again that all primes of $\mathrm{E}_{2}$ above $p$ split in $\mathrm{E}_{2} \cdot \mathrm{E}\left(G^{\prime}, X^{\prime}\right)$.

Finally, we show that $(G, X)$ may be chosen to satisfy the last condition. We may assume that $(G, X)$ already satisfies the first four conditions. In particular $Z_{G}$ is a torus, which splits over a tamely ramified extension of $\mathbb{Q}_{p}$. Since $(G, X)$ is of Hodge type, $Z_{G}$ splits over a CM field $F$. Let $F_{0}$ be the totally real subfield of $F$. Let $F_{1}$ be a quadratic imaginary field in which $p$ splits, and which is linearly disjoint from $F$, and set $F^{\prime}=F \cdot F_{1}$. The $F^{\prime}$ is a CM field, and we denote by $F_{0}^{\prime}$ its totally real subfield.

Let $T$ and $T_{0}$ be the tori whose $\mathbb{Q}$-points are given by $F^{\prime \times}$ and $\operatorname{ker}\left(F^{\prime \times} \rightarrow F_{0}^{\prime \times}\right)$ respectively. Fix an embedding $\overline{\mathbb{Q}} \hookrightarrow \overline{\mathbb{Q}}_{p}$. The action of complex conjugation on $\mathbb{X}_{\bullet}(T)$ does not coincide with that of any element of the inertia subgroup $I_{\mathbb{Q}_{p}}$, since the latter acts trivially on $F_{1}^{\times}$. In particular $\mathbb{X}_{\bullet}\left(T_{0}\right)_{I_{\mathbb{Q}_{p}}}=\mathbb{X}_{\bullet}(T)_{I_{\mathbb{Q}_{p}}}^{c=-1}$ is torsion free since $X_{*}(T)$ is an induced Galois module. Here $c$ denotes complex conjugation.

Since $Z_{G} / w_{h}\left(\mathbb{G}_{m}\right)(\mathbb{R})$ is contained in a compact real Lie group, $c$ acts by -1 on $\mathbb{X}^{\bullet}\left(Z_{G^{\text {der }}}\right)$. Hence for some integers $n, r$ there exists an embedding $\mathbb{X}^{\bullet}\left(Z_{G^{\text {der }}}\right) \hookrightarrow$ $\mathbb{X}^{\bullet}\left(T_{0}[n]^{r}\right)$. Denote by $T_{1}$, the pushout of

$$
1 \rightarrow T_{0}[n]^{r} \rightarrow T_{0}^{r} \stackrel{n}{\rightarrow} T_{0}^{r} \rightarrow 1
$$

by the corresponding map $T_{0}[n]^{r} \rightarrow Z_{G^{\text {der }}}$. Then $Z_{G^{\text {der }}} \subset T_{1}$, and $T_{1} / Z_{G^{\text {der }}} \cong T_{0}^{r}$. Let $\left(Z_{G}\right)_{0} \subset Z_{G}$ be the subtorus corresponding to $\mathbb{X}_{\bullet}\left(Z_{G}\right)^{c=-1} \subset \mathbb{X}_{\bullet}\left(Z_{G}\right)$. There is an embedding $\left(Z_{G}\right)_{0} \hookrightarrow T_{0}^{s}$ for some integer $s$. Set $G^{\prime}=\left(G \times T_{1} \times T_{0}^{s}\right) /\left(Z_{G^{\text {der }}} \times\right.$ $\left.\left(Z_{G}\right)_{0}\right)$, where $Z_{G^{\text {der }}} \times\left(Z_{G}\right)_{0}$ acts on $G$ via the multiplication $Z_{G^{\text {der }}} \times\left(Z_{G}\right)_{0} \rightarrow$ $\left(Z_{G}\right)_{0}$. Let $X^{\prime}$ be the $G^{\prime}$-conjugacy class induced by $X$. Then $\left(G^{\prime}, X^{\prime}\right)$ has the same reflex field as $(G, X)$, and satisfies the first four conditions of the Lemma. Moreover, one sees as above that $\left(G^{\prime}, X^{\prime}\right)$ is of Hodge type.

We have $G^{\prime \text { der }}=G^{\text {der }}$, and $\left(Z_{G^{\prime}}\right)_{0} / Z_{G^{\text {der }}}=\left(T_{1} \times T_{0}^{s}\right) / Z_{G^{\text {der }}}$ with $z \in Z_{G^{\text {der }}}$ acting by $\left(z, z^{-1}\right)$. Hence we have an exact sequence

$$
0 \rightarrow \mathbb{X}_{\bullet}\left(T_{0}^{s}\right) \rightarrow \mathbb{X}_{\bullet}\left(\left(Z_{G^{\prime}}\right)_{0} / Z_{G^{\text {der }}}\right) \rightarrow \mathbb{X}_{\bullet}\left(T_{1} / Z_{G^{\text {der }}}\right) \rightarrow 0 .
$$

In particular, $\mathbb{X}_{\bullet}\left(\left(Z_{G^{\prime}}\right)_{0} / Z_{G^{\text {der }}}\right)_{I_{\mathbb{Q}_{p}}}$ is torsion free. Finally $\mathbb{X}_{\bullet}\left(G^{\prime a b}\right)$ is an extension of $\mathbb{Z}$ by $\mathbb{X} \bullet\left(\left(Z_{G^{\prime}}\right)_{0} / Z_{G^{\text {der }}}\right)$, so $\mathbb{X}_{\bullet}\left(G^{\prime \text { ab }}\right)_{I_{\mathbb{Q}_{p}}}$ is torsion free.

Theorem 4.6.23. If $G_{2}$ splits over a tamely ramified extension of $\mathbb{Q}_{p}$, then there exists a Shimura datum of Hodge type $(G, X)$ such that the conditions of Corollary 4.6 .15 are satisfied, and all primes $v_{2} \mid p$ of $\mathrm{E}_{2}$ split completely in $\mathrm{E}^{\prime}$. In particular, for 
any prime $v_{2} \mid p$ of $\mathrm{E}_{2}$, the construction in Corollary 4.6 .15 gives rise to a $G_{2}\left(\mathbb{A}_{f}^{p}\right)$ equivariant $\mathcal{O}_{\mathrm{E}_{2}, v_{2}}$-scheme, $\mathscr{S}_{\mathrm{K}_{p}^{\circ}}\left(G_{2}, X_{2}\right)$ with the following properties.

(1) $\mathscr{S}_{\mathrm{K}_{2, p}^{\circ}}\left(G_{2}, X_{2}\right)$ is étale locally isomorphic to $\mathrm{M}_{G, X}^{\mathrm{loc}}$.

(2) If $p \nmid\left|\pi_{1}\left(G_{2}^{\text {der }}\right)\right|$, then $\mathscr{S}_{\mathrm{K}_{2, p}^{\circ}}\left(G_{2}, X_{2}\right)$ is étale locally isomorphic to $\mathrm{M}_{G_{2}, X_{2}}^{\mathrm{loc}}$.

(3) For any discrete valuation ring $R$ of mixed characteristic $0, p$ the map

$$
\mathscr{S}_{\mathrm{K}_{2, p}^{\circ}}\left(G_{2}, X_{2}\right)(R) \rightarrow \mathscr{S}_{\mathrm{K}_{2, p}^{\circ}}\left(G_{2}, X_{2}\right)(R[1 / p])
$$

is a bijection.

(4) If $\left(G_{2}^{\mathrm{ad}}, X^{\text {ad }}\right)$ has no factors of type $D^{\mathbb{H}}$, then $(G, X)$ can be chosen so that there exists a diagram of $\mathcal{O}_{E_{2}}$-schemes with $G_{2}\left(\mathbb{A}_{f}^{p}\right)$-action

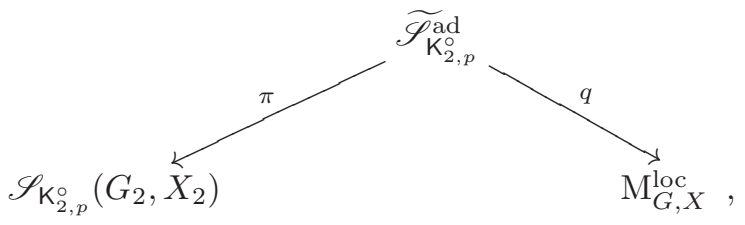

where $\pi$ is a $G_{2, \mathbb{Z}_{p}}^{\mathrm{ad}}$-torsor, $q$ is $G_{2, \mathbb{Z}_{p}}^{\mathrm{ado}}$-equivariant, and for any sufficiently small, compact open $\mathrm{K}_{2}^{p} \subset G\left(\mathbb{A}_{f}^{p}\right)$, the map $\mathscr{S}_{\mathrm{K}_{2, p}}^{\text {ad }} / \mathrm{K}_{2}^{p} \rightarrow \mathrm{M}_{G, X}^{\mathrm{loc}}$, induced by $q$ is smooth of relative dimension $\operatorname{dim} G_{2}^{\text {ad }}$.

In particular, if $\kappa^{\prime}$ is a finite extension of $\kappa\left(v_{2}\right)$, and $y \in \mathscr{S}_{\boldsymbol{K}_{2, p}^{\circ}}\left(\kappa^{\prime}\right)$, then there exists $z \in \mathrm{M}_{G, X}^{\mathrm{loc}}\left(\kappa^{\prime}\right)$ such that

$$
\mathcal{O}_{\mathscr{S}_{2, p}^{\circ}, y}^{\mathrm{h}}=\mathcal{O}_{\mathrm{M}_{G, X}^{\mathrm{loc},}, z}^{\mathrm{h}} \text {. }
$$

(5) If $G_{2, \mathbb{Q}_{p}}$ is unramified, and there exists $x_{2}^{\prime} \in \mathcal{B}\left(G_{2}, \mathbb{Q}_{p}\right)$ with $\mathcal{G}_{2, x_{2}^{\prime a d}}=$ $\mathcal{G}_{2, x_{2}^{\text {ad }}}^{\circ}$, then $(G, X)$ can be chosen so that the construction in Corollary 4.6 .15 applies with $x_{2}^{\prime}$ in place of $x_{2}$, and gives rise to an $\mathcal{O}_{\mathrm{E}_{2}, v_{2}}$-scheme $\mathscr{S}_{\mathrm{K}_{2, p}^{\circ}}\left(G_{2}, X_{2}\right)$ satisfying the conclusion of (4) above.

Proof. We apply Lemma 4.6 .22 and choose $(G, X)$ satisfying conditions (1)-(4) of that Lemma. As before, after conjugating $X$ by an element of $G^{\text {add }}(\mathbb{Q})$, we may assume that $X \subset X^{\text {ad }}=X_{2}^{\text {ad }}$ contains some connected component $X_{2}^{+}$of $X_{2}$. Then (2) and (4) imply that the conditions of Corollary 4.6.15 are satisfied, and (3) implies that $v_{2}$ extends to a place $v^{\prime}$ of $\mathrm{E}^{\prime}$ such that $E^{\prime}=E_{2, v_{2}}$. The claim (1) in the Theorem then follows from Corollary 4.6.18 The claim (2) follows by combining this with Proposition 2.2.7.

Let

$$
\mathscr{S}_{\mathrm{K}_{p}^{\prime}}\left(\mathrm{GSp}, S^{ \pm}\right)=\lim _{\leftarrow \mathrm{K}^{\prime p}} \mathscr{S}_{\mathrm{K}_{p}^{\prime} \mathrm{K}^{\prime p}}\left(\mathrm{GSp}, S^{ \pm}\right)
$$

where $\mathscr{S}_{\mathrm{K}_{p}^{\prime} \mathrm{K}^{\prime p}}\left(\mathrm{GSp}, S^{ \pm}\right)$is defined in 4.2.1. Then $\mathscr{S}_{\mathrm{K}_{p}^{\prime}}\left(\mathrm{GSp}, S^{ \pm}\right)$satisfies the extension property in (3), by the Néron-Ogg-Shafarevich criterion. Indeed a $R[1 / p]$ point of $\mathscr{S}_{K_{p}^{\prime}}\left(\mathrm{GSp}, S^{ \pm}\right)$defines an abelian variety over $R[1 / p]$, together with a trivialization of its $l$-adic Tate module for any $l \neq p$. Hence the abelian variety has good reduction, and the $R[1 / p]$-point comes from an $R$-point. Now (3) follows for $\left(G_{2}, X_{2}\right)$ of Hodge type and then of abelian type, by construction.

To see (4), we choose $(G, X)$ satisfying (5) of Lemma 4.6.22 Since in this case $\pi_{1}\left(G^{\text {der }}\right)=\{1\}$, we have $\pi_{1}(G)=\mathbb{X}_{\bullet}\left(G^{\text {ab }}\right)$, and $\pi_{1}(G)_{I_{\mathbb{Q}_{p}}}$ is torsion free. In particular, if $x \in \mathcal{B}\left(G, \mathbb{Q}_{p}\right)$ lifts $x_{2}^{\text {ad }}$, the Kottwitz map $\kappa_{G}$ is trivial on $\mathcal{G}_{x}\left(\mathbb{Z}_{p}^{\text {ur }}\right)$, 
and $\mathcal{G}_{x}=\mathcal{G}_{x}^{\circ}$. Hence the existence of the diagram in (4) of the Theorem follows by combining Corollary 4.6.18 with Lemma 4.6.2. The final claim in (4) follows by Lang's lemma.

For $(5)$, choose $x_{2}^{\prime} \in \mathcal{B}\left(G_{2}, \mathbb{Q}_{p}\right)$ such that $\mathcal{G}_{2, x_{2}^{\prime \text { ad }}}=\mathcal{G}_{2, x_{2}^{\text {ad }}}^{\circ}$. Then $\mathcal{G}_{2, x_{2}^{\prime}}\left(\mathbb{Z}_{p}^{\text {ur }}\right) \subset$ $G_{2}\left(\mathbb{Q}_{p}^{\text {ur }}\right)$ consists of those points which map to $\mathcal{G}_{2, x_{2}^{\prime a d}}\left(\mathbb{Z}_{p}^{\text {ur }}\right)$ and into the maximal bounded subgroup of $G_{2}^{\text {ab }}\left(\mathbb{Q}_{p}^{\text {ur }}\right)$. In particular $\mathcal{G}_{2, x_{2}^{\prime}}\left(\mathbb{Z}_{p}^{\text {ur }}\right) \subset \mathcal{G}_{2, x_{2}}\left(\mathbb{Z}_{p}^{\text {ur }}\right)$ and for any $g \in \mathcal{G}_{2, x_{2}^{\prime}}\left(\mathbb{Z}_{p}^{\mathrm{ur}}\right), \kappa_{G_{2}}(g)$ maps to 0 in $\pi_{1}\left(G_{2}^{\mathrm{ad}}\right)$ and $\mathbb{X}_{\bullet}\left(G_{2}^{\mathrm{ab}}\right)$. Hence $\kappa_{G_{2}}(g)=0$, and $\mathcal{G}_{2, x_{2}^{\prime}}=\mathcal{G}_{2, x_{2}}^{\circ}$.

Now choose $(G, X)$ satisfying (1)-(4) of Lemma 4.6.22. From the construction one sees easily that one may in fact choose $(G, X)$ so that $G_{\mathbb{Q}_{p}}$ is unramified. Choose $x \in \mathcal{B}\left(G, \mathbb{Q}_{p}\right)$ lifting $x_{2}^{\prime a d}$, The condition on $x_{2}^{\prime}$ implies that the Kottwitz map $\kappa_{G}: \mathcal{G}_{x}\left(\mathbb{Z}_{p}^{\text {ur }}\right) \rightarrow \pi_{1}(G)$ factors through $\mathbb{X}_{\bullet}\left(Z_{G}\right)$, and hence is trivial, as the latter group has no torsion. Hence $\mathcal{G}_{x}=\mathcal{G}_{x}^{\circ}$. Now (5) follows using the same argument as in (4).

Remark 4.6.25. a) If $K_{2}^{p} \subset G_{2}\left(\mathbb{A}_{f}^{p}\right)$ is sufficiently small, the conclusions of Theorem 4.6.23 about étale local structure also hold for the quotient $\mathscr{S}_{\mathrm{K}_{2}^{\circ}}\left(G_{2}, X_{2}\right)=$ $\mathscr{S}_{\mathrm{K}_{2, p}^{\circ}}(G, X) / \mathrm{K}_{2}^{p}$.

b) The form of Theorem 4.6 .23 is that a particular construction gives a model of the Shimura variety with parahoric level structure with the described properties. Unfortunately we do not know how to characterize the models constructed in Theorem 4.6.23, for example using an extension property as in the hyperspecial case. However, it seems to us that this should not be a problem in applications. For example, the methods of 52 should show that the models we construct are proper when the group $G^{\text {ad }}$ is anisotropic over $\mathbb{Q}$ (so the corresponding Shimura variety is proper). Secondly, the construction should be well adapted for applications involving computation of the zeta function (see 44) for the hyperspecial case.

c) Theorem 0.4 of the Introduction follows from Theorem4.6.23 (4), (5), by using Proposition 2.2.7 and Remark 4.2.14.

Corollary 4.6.26. Let $p>2$ and $(G, X)$ be a Shimura datum of abelian typ 4 with reflex field $\mathrm{E}$, such that $G$ splits over a tamely ramified extension of $\mathbb{Q}_{p}$. Let $x \in \mathcal{B}\left(G, \mathbb{Q}_{p}\right)$ and $\mathrm{K}_{p}^{\circ} \subset G\left(\mathbb{Q}_{p}\right)$ the corresponding compact open, parahoric subgroup. Suppose $v \mid p$ is a prime of $\mathrm{E}$, and assume that $\mathrm{K}^{p}$ is sufficiently small.

Then the special fibre of the integral model $\mathscr{S}_{\mathrm{K}}(G, X)$ over $\mathcal{O}_{\mathrm{E}, v}$ constructed above is reduced; the geometric analytic branches of the special fibre at each point are normal and Cohen-Macaulay.

If $x$ is a special vertex in $\mathcal{B}\left(G, \mathbb{Q}_{p}^{\text {ur }}\right)$ then the special fibre in normal (hence analytically unibranch at each point) and Cohen-Macaulay.

Proof. This follows from Theorem 4.6.23 combined with 59] Theorem 9.1 and Corollary 9.4 applied to the local model for the corresponding Hodge type group (denoted by $G$ in Theorem 4.6 .23 and its proof.) In particular, $p>2$ implies the assumption on the fundamental group needed in [59].

4.6.27. For a Shimura datum $\left(G_{2}, X_{2}\right)$ of abelian type, as in 4.6 .23 the construction of the integral model $\mathscr{S}_{K_{2}^{\circ}}\left(G_{2}, X_{2}\right)$ depends on the choice of Shimura datum

\footnotetext{
${ }^{7}$ Note that the group $G_{2}$ in Theorem 4.6 .23 is now denoted by $G$.
} 
$(G, X)$, as well as the choice of symplectic embedding $(G, X) \hookrightarrow\left(\mathrm{GSp}, S^{ \pm}\right)$. It seems reasonable to conjecture that the resulting integral model is independent of all choices, but we do not know how to prove this in general; by the argument in [54, §2], the extension property in 4.6.23 (3) is enough to guarantee only that two such models contain isomorphic open neighborhoods containing all generic points of the special fibre. We give show the independence in the special case:

Proposition 4.6.28. Let $(G, X)$ be as in Corollary 4.6.26, and suppose that $\mathrm{K}_{2, p}^{\circ}$ is a very special parahoric subgroup and that $G^{\text {ad }}$ is absolutely simple. Then the model $\mathscr{S}_{K_{2}^{\circ}}(G, X)$ does not depend on the choices made in its construction.

Proof. Consider any Shimura datum $(G, X)$, such that $G^{\text {ad }}$ is absolutely simple. Let $h \in X^{\text {ad }}$, and let $T \subset G_{\mathbb{C}}^{\text {ad }}$ be a maximal torus with $\mu_{h} \in X_{*}(T)$, and $B \supset T$ a Borel subgroup of $G$ for which $\mu_{h}^{-1}$ is dominant. We denote by $P \supset B$ the parabolic subgroup of $G$ corresponding to $\mu_{h}^{-1}$, and by $N \subset P$ the unipotent radical. Since $\mu_{h}$ is minuscule, and $G^{\text {ad }}$ is absolutely simple, there is exactly one simple root which is contained in Lie $(N)$, and it generates the space of characters $\mathbb{X}^{\bullet}(P)$ of $P$. For any compact open subgroup $\mathrm{K} \subset G\left(\mathbb{A}_{F}\right)$, any such character $\chi$ gives rise to a line bundle $\omega_{\chi}$ on $\operatorname{Sh}_{K}(G, X)$ which is defined over the reflex field [53]. The group $\mathbb{X} \bullet(P)=\mathbb{Z}$, has a unique generator $\chi_{0}$ such that $\omega_{\chi_{0}}$ is ample, and we write $\omega_{G}$ for $\omega_{\chi_{0}}$.

Now let $(G, X)$ be as in Corollary 4.6.26, and assume that $\mathrm{K}_{p}^{\circ}$ is very special. Then $\mathscr{S}_{\mathrm{K}^{\circ}}(G, X)$ has normal special fibre. We claim that any irreducible component of $\mathscr{S}_{\mathrm{K}^{\circ}}(G, X)$ has irreducible special fibre. From the construction, it suffices to prove this when $(G, X)$ is of Hodge type. By [52 there is an open embedding $\mathscr{S}_{\mathrm{K}^{\circ}}(G, X) \hookrightarrow \overline{\mathscr{S}}_{\mathrm{K}^{\circ}}(G, X)$ whose complement is a relative Cartier divisor, and such that $\overline{\mathscr{S}}_{\mathrm{K}^{\circ}}(G, X)$ has normal special fibre. This implies the claim, first for $\overline{\mathscr{S}}_{\mathrm{K}^{\circ}}(G, X)$ in place of $\mathscr{S}_{\mathrm{K}^{\circ}}(G, X)$ by Stein factorization, and then for $\mathscr{S}_{\mathrm{K}^{\circ}}(G, X)$.

In particular, any extension of a line bundle on $\operatorname{Sh}_{\mathrm{K}^{\circ}}(G, X)$ to $\mathscr{S}_{\mathrm{K}^{\circ}}(G, X)$ is unique, in the sense that any two extensions differ by an automorphism of $\omega_{G}$ which is a scalar on any irreducible component of $\operatorname{Sh}_{K^{\circ}}(G, X)$. We will first show that for some $n>0, \omega_{G}^{\otimes n}$ extends to an ample line bundle $\mathcal{L}$ on $\mathscr{S}_{\mathrm{k}^{\circ}}(G, X)$. For that one reduces easily to the case of $(G, X)$ of Hodge type. For any symplectic embedding $\iota:(G, X) \hookrightarrow\left(\mathrm{GSp}, S^{ \pm}\right), \iota^{*}\left(\omega_{\mathrm{GSp}}\right)$ is ample, and corresponds to a character of $P$. Hence it is equal to $\omega_{G}^{\otimes n}$ for some $n>0$. Since $\omega_{\mathrm{GSp}}$ has an ample extension, $\iota^{*}\left(\omega_{\mathrm{GSp}}\right)$ has an ample extension, by construction.

Now suppose that we have two (a priori) different models $\mathscr{S}=\mathscr{S}_{\mathrm{K}^{\circ}}(G, X)$ and $\mathscr{S}^{\prime}=\mathscr{S}_{\mathrm{K}^{\circ}}^{\prime}(G, X)$ of $\mathrm{Sh}_{\mathrm{K}^{\circ}}(G, X)$ with ample line bundles $\mathcal{L}$ and $\mathcal{L}^{\prime}$ which extend the same power $\omega_{G}^{\otimes n}$. As remarked above, $\mathscr{S}$ and $\mathscr{S}^{\prime}$ contain isomorphic open subschemes containing all generic points of the special fibres. Moreover, as above, we can assume that the isomorphism between these open subschemes lifts to an isomorphism between the corresponding restrictions of $\mathcal{L}$ and $\mathcal{L}^{\prime}$. Assume that $U$ is a common open neighborhood of $\mathscr{S}$ and $\mathscr{S}^{\prime}$, which contains the generic fibre and all generic points of the special fibres. Then, since $\mathscr{S}$ and $\mathscr{S}^{\prime}$ are normal, we have $\Gamma\left(\mathscr{S}, \mathcal{L}^{\otimes j}\right)=\Gamma\left(U, \omega_{G}^{\otimes n j}\right)=\Gamma\left(\mathscr{S}^{\prime}, \mathcal{L}^{\prime \otimes j}\right)$. Since both $\mathcal{L}$ and $\mathcal{L}^{\prime}$ are ample, this allows us to view both $\mathscr{S}$ and $\mathscr{S}^{\prime}$ as open subschemes of $\operatorname{Proj}(A)$, with $A=$ $\oplus_{j \geq 0} \Gamma\left(U, \omega_{G}^{\otimes n j}\right)$.

To show $\mathscr{S}=\mathscr{S}^{\prime}$, it is now enough to verify that $\mathscr{S}(k)=\mathscr{S}^{\prime}(k)$, where $k$ is an algebraic closure of $\mathbb{F}_{p}$. By flatness each $k$-valued point of $\mathscr{S}$ lifts to an $R$-valued 
point of $\mathscr{S}$ where $R$ is some strictly henselian discrete valuation ring of mixed characteristic 0, and similarly for $\mathscr{S}^{\prime}$. Since $\mathscr{S}_{\mathrm{K}_{p}^{\circ}}(G, X) \rightarrow \mathscr{S}$ is pro-étale, the $R$-valued point of $\mathscr{S}$ lifts to an $R$-valued point of $\mathscr{S}_{\mathrm{k}_{p}^{\circ}}(G, X)$, and similarly for $\mathscr{S}^{\prime}$ and $\mathscr{S}_{\mathrm{K}_{p}^{\circ}}^{\prime}(G, X)$. The result now follows using the extension property 4.6.23 (3) and the fact that $\operatorname{Proj}(A)$ is separated.

4.7. Nearby cycles. We now give some results about the nearby cycles of the integral models $\mathscr{S}_{\mathrm{K}^{\circ}}(G, X)$ over $\mathcal{O}_{\mathrm{E}, v}$ which can be obtained easily by combining the above with results of [59] about the nearby cycles of local models.

4.7.1. Let $l \neq p$ be a prime. Set $S=\operatorname{Spec}\left(\mathcal{O}_{E}\right), \eta=\operatorname{Spec}(E), s=\operatorname{Spec}\left(k_{E}\right)$, so that $(S, s, \eta)$ is a henselian trait. Let $\bar{E}$ be an algebraic closure of $E$, with residue field $k_{\bar{E}}$, and write $\bar{\eta}=\operatorname{Spec}(\bar{E}), \bar{s}=\operatorname{Spec}\left(k_{\bar{E}}\right)$ and $\bar{S}$ for the normalization of $S$ in $\bar{\eta}$. If $f: X \rightarrow S$ is a scheme of finite type and $\mathcal{F}$ in $D_{c}^{b}\left(X_{\eta}, \overline{\mathbb{Q}}_{l}\right)$ we set

$$
R \Psi(X, \mathcal{F})=\bar{i}_{*} R \bar{j}^{*}\left(\mathcal{F}_{\bar{\eta}}\right)
$$

for the "complex" of nearby cycles. Here $\bar{i}: X_{\bar{s}} \hookrightarrow X_{\bar{S}}$ and $\bar{j}: X_{\bar{\eta}} \hookrightarrow X_{\bar{S}}$ are the closed and open immersions of the geometric special and generic fibres and $\mathcal{F}_{\bar{\eta}}$ is the pull-back of $\mathcal{F}$ to $X_{\bar{\eta}}$. Recall that $R \Psi(X, \mathcal{F})$ is an object in $D_{c}^{b}\left(X_{\bar{s}}, \overline{\mathbb{Q}}_{l}\right)$ which supports a continuous action of $\Gamma_{E}=\operatorname{Gal}(\bar{\eta} / \eta)$ compatible with the action on $X_{\bar{s}}$ via $\Gamma_{E}=\operatorname{Gal}(\bar{\eta} / \eta) \rightarrow \operatorname{Gal}(\bar{s} / s)$. See 39 for more details. For a point $x \in X\left(\mathbb{F}_{q}\right), \mathbb{F}_{q} \supset k_{E}$, and corresponding geometric point $\bar{x}=\operatorname{Spec}(\bar{s}) \rightarrow X$, the inertia subgroup $I_{E} \subset \Gamma_{E}$ acts on the stalk $R \Psi(X, \mathcal{F})_{\bar{x}}$ and one can define 62 the semi-simple trace of Frobenius

$$
\operatorname{Tr}^{s s}\left(\operatorname{Frob}_{x}, R \Psi(X, \mathcal{F})_{\bar{x}}\right) .
$$

We will denote $R \Psi\left(X, \overline{\mathbb{Q}}_{l}\right)$ simply by $R \Psi^{X}$.

We refer the reader to [32, [34, 37, for more details, additional references and background on nearby cycles of integral models of Shimura varieties.

4.7.2. For the following result, the notation and hypotheses are as in Corollary 4.6.26 In addition, we denote by $F \subset \bar{E}$ a tamely ramified, finite extension of $E$ over which $G$ splits. 8 In particular, we have $x \in \mathcal{B}\left(G, \mathbb{Q}_{p}\right)$, and the group schemes $\mathcal{G}=\mathcal{G}_{x}$ and $\mathcal{G}^{\text {ad }}=\mathcal{G}_{x^{\text {ad }}}^{\text {ad }}$. For notational simplicity, we set $\mathscr{S}=\mathscr{S}_{\mathrm{k}}(G, X)$.

Corollary 4.7.3. The inertia subgroup $I_{F}$ of $\operatorname{Gal}(\bar{E} / F)$ acts unipotently on all the stalks $R \Psi_{\bar{z}}^{\mathscr{S}}$, for $\bar{z}$ in $\mathscr{S}\left(k_{\bar{E}}\right)$. If $x \in \mathcal{B}\left(G, \mathbb{Q}_{p}\right)$ is a very special vertex, then $I_{F}$ acts trivially on all the stalks $R \Psi_{\bar{z}}^{\mathscr{S}}, \bar{z}$ as above.

Proof. This follows from Corollary 4.6.26, 59] Theorems 10.9 and 10.12, and the fact that the stalk of the nearby cycles at $\bar{z}$ with its inertia action depends only on the strict henselization of the local ring at $\bar{z}$.

4.7.4. Now suppose that there exists a $G\left(\mathbb{A}_{f}^{p}\right)$-equivariant local model diagram

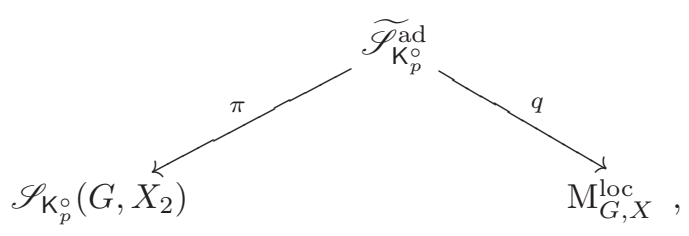

\footnotetext{
${ }^{8}$ Note that the conditions of Corollary 4.6 .26 imply that $E$ is tamely ramified over $\mathbb{Q}_{p}$, so $F$ is also.
} 
where $\pi$ a $\mathcal{G}^{\text {ado }}$-torsor, $q$ is a $\mathcal{G}^{\text {ado }}$-equivariant map, any sufficiently small compact open $\mathrm{K}^{p} \subset G\left(\mathbb{A}_{f}^{p}\right)$, acts freely on $\widetilde{\mathscr{S}_{\mathrm{K}}^{\circ}}$, and the map $\widetilde{\mathscr{S}_{\mathrm{K}}^{\circ}} \underset{\mathrm{K}}{\mathrm{ad}} \rightarrow \mathrm{M}_{G, X}^{\mathrm{loc}}$ induced by $q$ is smooth of relative dimension $\operatorname{dim} G^{\text {ad }}$. Such a diagram exists when $p \nmid\left|\pi_{1}\left(G^{\text {der }}\right)\right|$ and, either $\left(G^{\text {ad }}, X^{\text {ad }}\right)$ has no factors of type $D^{\mathbb{H}}$, or $G$ is unramified over $\mathbb{Q}_{p}$ and $\mathrm{K}_{p}^{\circ}$ is contained in a hyperspecial, by Theorem 0.4 .

Suppose $y \in \mathscr{S}\left(\mathbb{F}_{q}\right)$, where $\mathbb{F}_{q} \supset k_{E}$. Using Lang's Lemma we see that there is a point $w \in \mathrm{M}_{G, X}^{\text {loc }}\left(\mathbb{F}_{q}\right)$, well-defined up to the action of $\mathcal{G}^{\text {ado }}\left(\mathbb{F}_{q}\right)$, such that we have an isomorphism of henselizations

$$
\mathcal{O}_{\mathscr{S}, y}^{\mathrm{h}} \simeq \mathcal{O}_{\mathrm{M}_{G, X}^{\mathrm{loc}}, w}^{\mathrm{h}} .
$$

This in turn implies an equality of semi-simple traces

$$
\operatorname{Tr}^{s s}\left(\operatorname{Frob}_{y}, R \Psi_{\bar{y}}^{\mathscr{S}}\right)=\operatorname{Tr}^{s s}\left(\operatorname{Frob}_{w}, R \Psi_{\bar{w}}^{\mathrm{M}_{G, X}^{\mathrm{loc}}}\right) .
$$

Set $r=\left[\mathbb{F}_{q}: k_{E}\right]$. Consider the function

$$
\psi_{r}: \mathscr{S}\left(\mathbb{F}_{q}\right) \rightarrow \overline{\mathbb{Q}}_{l} ; \quad \psi_{r}(y):=\operatorname{Tr}^{s s}\left(\operatorname{Frob}_{y}, R \Psi_{\bar{y}}^{\mathscr{S}}\right) .
$$

(The function $\psi_{r}$ appears in the Langlands-Kottwitz method for the calculation of the factor at $v$ of the semi-simple zeta function of the Shimura variety $\operatorname{Sh}_{K^{\circ}}(G, X)$.) By (4.7.7) $\psi_{r}$ factors as a composition

$$
\mathscr{S}\left(\mathbb{F}_{q}\right) \stackrel{q}{\rightarrow} \mathcal{G}^{\circ}\left(\mathbb{F}_{q}\right) \backslash \mathrm{M}_{G, X}^{\mathrm{loc}}\left(\mathbb{F}_{q}\right) \stackrel{\varphi_{r}}{\longrightarrow} \overline{\mathbb{Q}}_{l}
$$

where

$$
\varphi_{r}: \mathrm{M}_{G, X}^{\mathrm{loc}}\left(\mathbb{F}_{q}\right) \rightarrow \overline{\mathbb{Q}}_{l} ; \quad \varphi_{r}(w)=\operatorname{Tr}^{s s}\left(\operatorname{Frob}_{y}, R \Psi_{\bar{w}}^{\mathrm{M}_{G}^{\mathrm{loc}}, X}\right) .
$$

4.7.8. In [59] there is a construction of a reductive group $G^{\prime}$ over $\mathbb{F}_{p}((t))$, resp. a parahoric subgroup scheme $\mathcal{G}^{\prime}$ over $\mathbb{F}_{p} \llbracket t \rrbracket$, of the "same type" as $G$, resp. $\mathcal{G}^{\circ}$; in particular, we can identify the special fibres of $\mathcal{G}^{\circ}$ and $\mathcal{G}^{\prime}$ over $\mathbb{F}_{p}$. We have an $\mathcal{G}^{\prime}\left(\mathbb{F}_{q} \llbracket t \rrbracket\right)$-equivariant embedding $\mathrm{M}_{G, X}^{\mathrm{loc}}\left(\mathbb{F}_{q}\right) \subset G^{\prime}\left(\mathbb{F}_{q}((t))\right) / \mathcal{G}^{\prime}\left(\mathbb{F}_{q} \llbracket t \rrbracket\right)$, with $\mathcal{G}^{\prime}\left(\mathbb{F}_{q} \llbracket t \rrbracket\right)$ acting on $\mathrm{M}_{G, X}^{\text {loc }}$ via $\mathcal{G}^{\circ}\left(\mathbb{F}_{q}\right)$. Set $\mathrm{P}_{r}^{\prime}=\mathcal{G}^{\prime}\left(\mathbb{F}_{q} \llbracket t \rrbracket\right)$. We have

$$
\mathcal{G}^{\circ}\left(\mathbb{F}_{q}\right) \backslash \mathrm{M}_{G, X}^{\mathrm{loc}}\left(\mathbb{F}_{q}\right) \subset \mathrm{P}_{r}^{\prime} \backslash G^{\prime}\left(\mathbb{F}_{q}((t))\right) / \mathrm{P}_{r}^{\prime} .
$$

We again denote by $\varphi_{r}$ the extension by 0 of $\varphi_{r}$ to $G^{\prime}\left(\mathbb{F}_{q}((t))\right) / \mathrm{P}_{r}^{\prime}$. Then $\varphi_{r}$ is $\mathrm{P}_{r}^{\prime}$-equivariant and so it gives

$$
\varphi_{r} \in \mathcal{H}_{r}\left(G^{\prime}, \mathcal{G}^{\prime}\right)=C_{c}\left(\mathrm{P}_{r}^{\prime} \backslash G^{\prime}\left(\mathbb{F}_{q}((t))\right) / \mathrm{P}_{r}^{\prime}\right)
$$

in the parahoric Hecke algebra of compactly supported $\mathrm{P}_{r}^{\prime}$-bivariant locally constant $\overline{\mathbb{Q}}_{l}$-valued functions on $G^{\prime}\left(\mathbb{F}_{q}((t))\right)$ under convolution (see [59] $\S 10.4 .2$.). By loc. cit., Theorem 10.14, for all $r \geq 1$, the function $\varphi_{r}$ belongs to the center of the Hecke algebra $\mathcal{H}_{r}\left(G^{\prime}, \mathcal{G}^{\prime}\right)$.

4.7.9. Let us now assume in addition that $G$ is unramified over $\mathbb{Q}_{p}, p \nmid\left|\pi_{1}\left(G^{\text {der }}\right)\right|$, and that either $\left(G^{\mathrm{ad}}, X^{\mathrm{ad}}\right)$ has no factor of type $D^{\mathbb{H}}$, or $\mathrm{K}_{p}^{\circ}$ is contained in a hyperspecial. Then we can apply the above discussion to the local model diagram given by Theorem $4.6 .23(4),(5)$.

The extension $E / \mathbb{Q}_{p}$ is unramified; denote by $E_{r} \subset L$ the unramified extension of $E$ of degree $r=\left[\mathbb{F}_{q}: k_{E}\right]$ with residue field $\mathbb{F}_{q}$ and by $\mathcal{O}_{r}=W\left(\mathbb{F}_{q}\right)$ the ring of integers of $E_{r}$ Set $P_{r}=\mathcal{G}^{\circ}\left(\mathcal{O}_{r}\right), \mathrm{P}_{r}^{\prime}=\mathcal{G}^{\prime}\left(\mathbb{F}_{q} \llbracket t \rrbracket\right)$. By the construction of $G^{\prime}$, for each $r \geq 1$, there is a natural bijection

$$
\mathrm{P}_{r}^{\prime} \backslash G^{\prime}\left(\mathbb{F}_{q}((t))\right) / \mathrm{P}_{r}^{\prime} \cong P_{r} \backslash G\left(E_{r}\right) / P_{r}
$$


which gives

$$
\mathcal{G}^{\circ}\left(\mathbb{F}_{q}\right) \backslash \mathrm{M}_{G, X}^{\mathrm{loc}}\left(\mathbb{F}_{q}\right) \hookrightarrow P_{r} \backslash G\left(E_{r}\right) / P_{r} .
$$

Using this, we can view $\varphi_{r}: \mathcal{G}^{\circ}\left(\mathbb{F}_{q}\right) \backslash \mathrm{M}_{G, X}^{\mathrm{loc}}\left(\mathbb{F}_{q}\right) \rightarrow \overline{\mathbb{Q}}_{l}$ as an element of the parahoric Hecke algebra $C_{c}\left(P_{r} \backslash G\left(E_{r}\right) / P_{r}\right)$. Set $d=\operatorname{dim}\left(\operatorname{Sh}_{K^{\circ}}(G, X)\right)$ and let $\mu$ be a cocharacter in the conjugacy class of $\mu_{h}$.

Theorem 4.7.11. (Kottwitz's conjecture) Suppose that $(G, X)$ is of abelian type with $G$ unramified, $p \nmid\left|\pi_{1}\left(G^{\mathrm{der}}\right)\right|$, and either $\left(G^{\mathrm{ad}}, X^{\mathrm{ad}}\right)$ has no factor of type $D^{\mathbb{H}}$, or $\mathrm{K}_{p}^{\circ}$ is contained in a hyperspecial. For $y \in \mathscr{S}\left(\mathbb{F}_{q}\right)$, we have

$$
\operatorname{Tr}^{s s}\left(\operatorname{Frob}_{y}, R \Psi_{\bar{y}}^{\mathscr{S}}\right)=q^{d / 2} z_{\mu, r}(w)
$$

where $w \in \mathrm{M}_{G, X}^{\mathrm{loc}}$ corresponds to $y$ and $z_{\mu, r}$ is the Bernstein function attached to $\mu$ in the center of the parahoric Hecke algebra $C_{c}\left(P_{r} \backslash G\left(E_{r}\right) / P_{r}\right)$.

Proof. See [51] (also the work of Haines [35, 34, 36]) for the definition of the Bernstein function $z_{\mu, r}$.

Let us pick an alcove in the apartment of the standard split torus $S$ (cf. 2.1.4) whose closure contains $x$ and let $x_{0}$ be a hyperspecial vertex in the same closure. This alcove defines an Iwahori group scheme $\mathcal{I}^{\prime}$ over $\mathbb{F} \llbracket t \rrbracket$. Set $\boldsymbol{I}_{r}^{\prime}=\mathcal{I}^{\prime}\left(\mathbb{F}_{q} \llbracket t \rrbracket\right)$ so that $\mathrm{I}_{r}^{\prime} \subset \mathrm{P}_{r}^{\prime}$. We also set $\mathrm{K}_{r}^{\prime}=\mathcal{G}_{0}^{\prime}\left(\mathbb{F}_{q} \llbracket t \rrbracket\right)$, where $\mathcal{G}_{0}^{\prime}$ is the reductive group scheme corresponding to $x_{0}$. Then $\mathrm{K}_{r}^{\prime}$ is a maximal compact subgroup of $G^{\prime}\left(\mathbb{F}_{q}((t))\right)$ and we also have $\mathrm{I}_{r}^{\prime} \subset \mathrm{K}_{r}^{\prime}$.

By [59] Theorem 10.16, for each $r \geq 1$, the function $\varphi_{r}$ is the unique element of the center $\mathcal{Z}\left(C_{c}\left(\mathrm{P}_{r}^{\prime} \backslash G^{\prime}\left(\mathbb{F}_{q}((t))\right) / \mathrm{P}_{r}^{\prime}\right)\right)$ whose image under the Bernstein isomorphism $\left(-* 1_{\mathrm{K}_{r}^{\prime}}\right) \cdot\left(-* 1_{\mathrm{P}_{r}^{\prime}}\right)^{-1}$ obtained by composing the convolutions (35. Theorem 3.1.1)

$$
\begin{aligned}
& -* 1_{\mathrm{P}_{r}^{\prime}}: \mathcal{Z}\left(C_{c}\left(\mathrm{I}_{r}^{\prime} \backslash G^{\prime}\left(\mathbb{F}_{q}((t))\right) / \mathrm{I}_{r}^{\prime}\right)\right) \stackrel{\sim}{\rightarrow} \mathcal{Z}\left(C_{c}\left(\mathrm{P}_{r}^{\prime} \backslash G^{\prime}\left(\mathbb{F}_{q}((t))\right) / \mathrm{P}_{r}^{\prime}\right)\right), \\
& -* 1_{\mathrm{K}_{r}^{\prime}}: \mathcal{Z}\left(C_{c}\left(\mathrm{I}_{r}^{\prime} \backslash G^{\prime}\left(\mathbb{F}_{q}((t))\right) / \mathrm{I}_{r}^{\prime}\right)\right) \stackrel{\sim}{\longrightarrow} C_{c}\left(\mathrm{~K}_{r}^{\prime} \backslash G^{\prime}\left(\mathbb{F}_{q}((t))\right) / \mathrm{K}_{r}^{\prime}\right),
\end{aligned}
$$

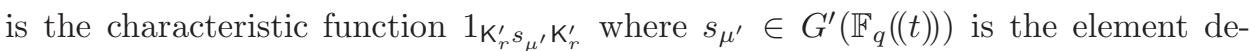
termined by the coweight $\mu^{\prime}$ of $G^{\prime}$ which corresponds to $\mu$ as in 59. (Note that the result in loc. cit. is given for the intersection complex $\overline{\mathbb{Q}}_{l}[d](d / 2)$.) It follows from the compatibility of the Bernstein and Satake isomorphisms that $\varphi_{r}$ is $q^{d / 2} z_{\mu^{\prime}, r}^{\prime}$, where $z_{\mu^{\prime}, r}^{\prime}$ is the Bernstein function in the center of the parahoric Hecke algebra $C_{c}\left(\mathrm{P}_{r}^{\prime} \backslash G^{\prime}\left(\mathbb{F}_{q}((t))\right) / \mathrm{P}_{r}^{\prime}\right)$. It remains to note that 44.7.10) induces an algebra isomorphism

$$
C_{r}\left(\mathrm{P}_{r}^{\prime} \backslash G^{\prime}\left(\mathbb{F}_{q}((t))\right) / \mathrm{P}_{r}^{\prime}\right) \cong C_{c}\left(P_{r} \backslash G\left(E_{r}\right) / P_{r}\right)
$$

which takes the Bernstein function $z_{\mu^{\prime}, r}^{\prime}$ to $z_{\mu, r}$ and the result then follows from (4.7.7).

\section{REFERENCES}

[1] S. Anantharaman, Schémas en groupes, espaces homogènes et espaces algébriques sur une base de dimension 1, Sur les groupes algébriques, Soc. Math. France, Paris, 1973, pp. 5-79. Bull. Soc. Math. France, Mém. 33.

[2] E. Bayer-Fluckiger and R. Parimala, Galois cohomology of the classical groups over fields of cohomological dimension $\leq 2$, Invent. Math. 122 (1995), no. 2, 195-229.

[3] Classical groups and the Hasse principle, Ann. of Math. (2) 147 (1998), no. 3, 651-693.

[4] P. Berthelot and A. Ogus, F-isocrystals and de Rham cohomology. I, Invent. Math. 72 (1983), no. 2, 159-199. 
[5] D. Blasius, A p-adic property of Hodge classes on abelian varieties, Motives (Seattle, WA, 1991), Proc. Sympos. Pure Math., vol. 55, Amer. Math. Soc., Providence, RI, 1994, pp. 293308.

[6] S. Bosch, W. Lütkebohmert, and M. Raynaud, Néron models, Ergebnisse der Mathematik und ihrer Grenzgebiete (3) [Results in Mathematics and Related Areas (3)], vol. 21, SpringerVerlag, Berlin, 1990.

[7] N. Bourbaki, Lie groups and Lie algebras. Chapters 7-9, Elements of Mathematics (Berlin), Springer-Verlag, Berlin, 2005, Translated from the 1975 and 1982 French originals by Andrew Pressley.

[8] C. Breuil, Groupes p-divisibles, groupes finis et modules filtrés, Ann. of Math. (2) 152 (2000), no. 2, 489-549.

[9] M. Broshi, G-torsors over a Dedekind scheme, J. Pure Appl. Algebra 217 (2013), no. 1, 11-19.

[10] F. Bruhat and J. Tits, Groupes réductifs sur un corps local, Inst. Hautes Études Sci. Publ. Math. (1972), no. 41, 5-251.

[11] - Groupes réductifs sur un corps local. II. Schémas en groupes. Existence d'une donnée radicielle valuée, Inst. Hautes Études Sci. Publ. Math. (1984), no. 60, 197-376.

[12] _ Schémas en groupes et immeubles des groupes classiques sur un corps local, Bull. Soc. Math. France 112 (1984), no. 2, 259-301.

[13] Schémas en groupes et immeubles des groupes classiques sur un corps local. II. Groupes unitaires, Bull. Soc. Math. France 115 (1987), no. 2, 141-195.

[14] J.-L. Colliot-Thélène, Un théorème de finitude pour le groupe de Chow des zéro-cycles d'un groupe algébrique linéaire sur un corps p-adique, Invent. Math. 159 (2005), no. 3, 589-606.

[15] _ Résolutions flasques des groupes linéaires connexes, J. Reine Angew. Math. 618 (2008), 77-133.

[16] J.-L. Colliot-Thélène and J.-J. Sansuc, Fibrés quadratiques et composantes connexes réelles, Math. Ann. 244 (1979), no. 2, 105-134.

[17] B. Conrad, Reductive group schemes, Notes for the SGA3 Summer School, Luminy 2011, http://math.stanford.edu/ ${ }^{\sim}$ conrad/papers/luminysga3.pdf

[18] A. J. de Jong, The moduli spaces of principally polarized abelian varieties with $\Gamma_{0}(p)$-level structure, J. Algebraic Geom. 2 (1993), no. 4, 667-688.

[19] _ Crystalline Dieudonné module theory via formal and rigid geometry, Inst. Hautes Études Sci. Publ. Math. (1995), no. 82, 5-96 (1996).

[20] P. Deligne, Variétés de Shimura: interprétation modulaire, et techniques de construction de modèles canoniques, Automorphic forms, representations and $L$-functions (Proc. Sympos. Pure Math., Oregon State Univ., Corvallis, Ore., 1977), Part 2, Proc. Sympos. Pure Math., XXXIII, Amer. Math. Soc., Providence, R.I., 1979, pp. 247-289.

[21] P. Deligne and G. Pappas, Singularités des espaces de modules de Hilbert, en les caractéristiques divisant le discriminant, Compositio Math. 90 (1994), no. 1, 59-79.

[22] B. Edixhoven, Néron models and tame ramification, Compositio Math. 81 (1992), no. 3, 291-306.

[23] G. Faltings and C.-L. Chai, Degeneration of abelian varieties, Ergebnisse der Mathematik und ihrer Grenzgebiete (3) [Results in Mathematics and Related Areas (3)], vol. 22, SpringerVerlag, Berlin, 1990, With an appendix by David Mumford.

[24] O. Gabber and F. Orgogozo, Sur la p-dimension des corps, Invent. Math. 174 (2008), no. 1, 47-80.

[25] D. Gaitsgory, Construction of central elements in the affine Hecke algebra via nearby cycles, Invent. Math. 144 (2001), no. 2, 253-280.

[26] W. T. Gan and J.-K. Yu, Schémas en groupes et immeubles des groupes exceptionnels sur un corps local. I. Le groupe $G_{2}$, Bull. Soc. Math. France 131 (2003), no. 3, 307-358.

[27] - Schémas en groupes et immeubles des groupes exceptionnels sur un corps local. II. Les groupes $F_{4}$ et $E_{6}$, Bull. Soc. Math. France 133 (2005), no. 2, 159-197.

[28] P. Gille, Cohomologie galoisienne des groupes quasi-déployés sur des corps de dimension cohomologique $\leq 2$, Compositio Math. 125 (2001), no. 3, 283-325.

[29] _ Torseurs sur la droite affine, Transform. Groups 7 (2002), no. 3, 231-245.

[30] _ Serre's conjecture II: a survey, Quadratic forms, linear algebraic groups, and cohomology, Dev. Math., vol. 18, Springer, New York, 2010, pp. 41-56. 
[31] U. Görtz, On the flatness of local models for the symplectic group, Adv. Math. 176 (2003), no. $1,89-115$

[32] U. Görtz and T. Haines, The Jordan-Hölder series for nearby cycles on some Shimura varieties and affine flag varieties, J. Reine Angew. Math. 609 (2007), 161-213.

[33] A. Grothendieck, Le groupe de Brauer. I, II, III., Dix Exposés sur la Cohomologie des Schémas, North-Holland, Amsterdam, 1968, pp. 46-188.

[34] T. Haines, Introduction to Shimura varieties with bad reduction of parahoric type, Harmonic analysis, the trace formula, and Shimura varieties, Clay Math. Proc., vol. 4, Amer. Math. Soc., Providence, RI, 2005, pp. 583-642.

[35] _ The base change fundamental lemma for central elements in parahoric Hecke algebras, Duke Math. J. 149 (2009), no. 3, 569-643. Corrigendum, on the author's web page.

[36] _ The stable Bernstein center and test functions for Shimura varieties, Automorphic Forms and Galois Representations (London Mathematical Society Lecture Notes Series: 415), vol. 2, London Mathematical Society, 2014, pp. 118-186.

[37] T. Haines and B. C. Ngô, Nearby cycles for local models of some Shimura varieties, Compositio Math. 133 (2002), no. 2, 117-150.

[38] T. Haines and M. Rapoport, On parahoric subgroups, Appendix to [57, 2008.

[39] L. Illusie, Autour du théorème de monodromie locale, Astérisque (1994), no. 223, 9-57, Périodes $p$-adiques (Bures-sur-Yvette, 1988).

[40] L. Illusie, Y. Laszlo, and F. Orgogozo, Travaux de Gabber sur l'uniformisation locale et la cohomologie etale des schemas quasi-excellents. Seminaire a l'Ecole polytechnique 2006-2008, arXiv: $1207.3648 \mathrm{v} 1$.

[41] J. C. Jantzen, Representations of algebraic groups, second ed., Mathematical Surveys and Monographs, vol. 107, American Mathematical Society, Providence, RI, 2003.

[42] N. Katz, Travaux de Dwork, Séminaire Bourbaki, 24ème année (1971/1972), Exp. No. 409, (1973), 167-200. Lecture Notes in Math., Vol. 317.

[43] M. Kisin, Integral models for Shimura varieties of abelian type, J. Amer. Math. Soc. 23 (2010), no. 4, 967-1012.

[44], mod $p$ points on Shimura varieties of abelian type, J. Amer. Math. Soc. 30 (2017), no. 3, 819-914.

[45] R. Kottwitz, Shimura varieties and twisted orbital integrals, Math. Ann. 269 (1984), no. 3, 287-300.

[46] _ Points on some Shimura varieties over finite fields, J. Amer. Math. Soc. 5 (1992), no. $2,373-444$.

[47] T. Kuzumaki, The cohomological dimension of the quotient field of the two-dimensional complete local domain, Compositio Math. 79 (1991), no. 2, 157-167.

[48] E. Landvogt, Some functorial properties of the Bruhat-Tits building, J. Reine Angew. Math. 518 (2000), 213-241.

[49] R. P. Langlands, Some contemporary problems with origins in the Jugendtraum, Mathematical developments arising from Hilbert problems (Proc. Sympos. Pure Math., Vol. XXVIII, Northern Illinois Univ., De Kalb, Ill., 1974), Amer. Math. Soc., Providence, R. I., 1976, pp. 401-418.

[50] E. Lau, Relations between Dieudonné displays and crystalline Dieudonné theory, Algebra Number Theory 8 (2014), no. 9, 2201-2262.

[51] G. Lusztig, Singularities, character formulas, and a q-analog of weight multiplicities, Analysis and topology on singular spaces, II, III (Luminy, 1981), Astérisque, vol. 101, Soc. Math. France, Paris, 1983, pp. 208-229.

[52] K. Madapusi Pera, Toroidal compactifications of integral models of Shimura varieties of Hodge type., Preprint. arXiv:1211.1731

[53] J. S. Milne, Canonical models of (mixed) Shimura varieties and automorphic vector bundles, Automorphic forms, Shimura varieties, and L-functions, Vol. I (Ann Arbor, MI, 1988), Perspect. Math., vol. 10, Academic Press, Boston, MA, 1990, pp. 283-414.

[54] - The points on a Shimura variety modulo a prime of good reduction, The zeta functions of Picard modular surfaces, Univ. Montréal, Montreal, QC, 1992, pp. 151-253.

[55] L. Moret-Bailly, Groupes de Picard et problèmes de Skolem. I, II, Ann. Sci. École Norm. Sup. (4) 22 (1989), no. 2, 161-179, 181-194.

[56] G. Pappas, On the arithmetic moduli schemes of PEL Shimura varieties, J. Algebraic Geom. 9 (2000), no. 3, 577-605. 
[57] G. Pappas and M. Rapoport, Twisted loop groups and their affine flag varieties, Adv. Math. 219 (2008), no. 1, 118-198, With an appendix by T. Haines and Rapoport.

[58] G. Pappas, M. Rapoport, and B. Smithling, Local models of Shimura varieties, I. Geometry and combinatorics, Handbook of moduli. Vol. III, Adv. Lect. Math. (ALM), vol. 26, Int. Press, Somerville, MA, 2013, pp. 135-217.

[59] G. Pappas and X. Zhu, Local models of Shimura varieties and a conjecture of Kottwitz, Invent. Math. 194 (2013), no. 1, 147-254.

[60] G. Prasad and J-K. Yu, On finite group actions on reductive groups and buildings, Invent. Math. 147 (2002), no. 3, 545-560.

[61] _ On quasi-reductive group schemes, J. Algebraic Geom. 15 (2006), no. 3, 507-549, With an appendix by Brian Conrad.

[62] M. Rapoport, On the bad reduction of Shimura varieties, Automorphic forms, Shimura varieties, and $L$-functions, Vol. II (Ann Arbor, MI, 1988), Perspect. Math., vol. 11, Academic Press, Boston, MA, 1990, pp. 253-321.

[63] _ A guide to the reduction modulo $p$ of Shimura varieties, Astérisque (2005), no. 298, 271-318, Automorphic forms. I.

[64] M. Rapoport and Th. Zink, Period spaces for p-divisible groups, Annals of Mathematics Studies, vol. 141, Princeton University Press, Princeton, NJ, 1996.

[65] M. Raynaud and L. Gruson, Critères de platitude et de projectivité. Techniques de "platification" d'un module, Invent. Math. 13 (1971), 1-89.

[66] I. Satake, Symplectic representations of algebraic groups satisfying a certain analyticity condition, Acta Math. 117 (1967), 215-279.

[67] J.-P. Serre, Galois Cohomology, fifth ed., Lecture Notes in Mathematics, vol. 5, SpringerVerlag, Berlin, 1994

[68] C. S. Seshadri, Triviality of vector bundles over the affine space $K^{2}$, Proc. Nat. Acad. Sci. U.S.A. 44 (1958), 456-458.

[69] J. Tits, Représentations linéaires irréductibles d'un groupe réductif sur un corps quelconque, J. Reine Angew. Math. 247 (1971), 196-220.

[70] _ Reductive groups over local fields, Automorphic forms, representations and $L$ functions (Proc. Sympos. Pure Math., Oregon State Univ., Corvallis, Ore., 1977), Part 1, Proc. Sympos. Pure Math., XXXIII, Amer. Math. Soc., Providence, R.I., 1979, pp. 29-69.

[71] J.-P. Wintenberger, Existence de F-cristaux avec structures supplémentaires, Adv. Math. 190 (2005), no. 1, 196-224.

[72] J.-K. Yu, Smooth models associated to concave functions in Bruhat-Tits theory,, Preprint, 2002.

[73] Th. Zink, A Dieudonné theory for p-divisible groups, Class field theory-its centenary and prospect (Tokyo, 1998), Adv. Stud. Pure Math., vol. 30, Math. Soc. Japan, Tokyo, 2001, pp. 139-160.

[74] _ Windows for displays of p-divisible groups, Moduli of abelian varieties (Texel Island, 1999), Progr. Math., vol. 195, Birkhäuser, Basel, 2001, pp. 491-518.

[75] — The display of a formal p-divisible group, Astérisque (2002), no. 278, 127-248, Cohomologies $p$-adiques et applications arithmétiques, I.

Dept. of Mathematics, Harvard University, Cambridge, MA 02138, USA

E-mail address: kisin@math.harvard.edu

Dept. of Mathematics, Michigan State University, E. Lansing, Mi 48824, USA

E-mail address: pappas@math.msu.edu 